\title{
Human induced pluripotent stem cell models used in the study of doxorubicin-induced cardiomyopathy
}

\author{
Dissertation \\ for the award of the degree \\ "Doctor of Philosophy" \\ Division of Mathematics and Natural Sciences \\ of the Georg-August-Universität Göttingen
}

within the doctoral program Molecular Medicine

of the Georg-August University School of Science (GAUSS)

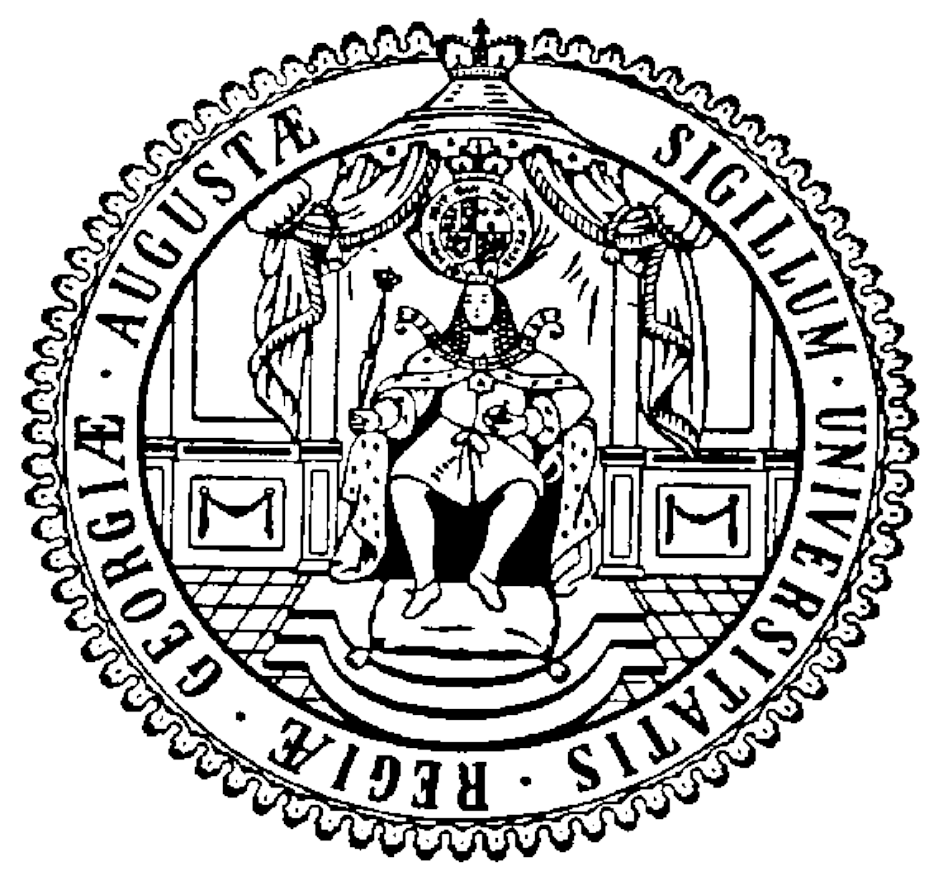

submitted by

Andreas Maus

born in Eichstätt, Germany

Göttingen 2020 


\section{Members of the examination board}

PD Dr. rer. nat. Katrin Streckfuß-Bömeke (supervisor and first reviewer)

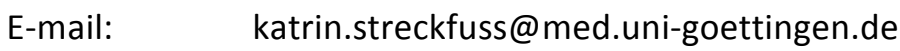

Postal address: Department of Cardiology and Pneumonology, University Medical Centre Göttingen Georg-August-University Göttingen, Robert-Koch-Straße 40, 37075 Göttingen

Prof. Dr. Ajay Shah (co-supervisor)

E-mail: $\quad$ ajay.shah@kcl.ac.uk

Postal address: School of Cardiovascular Medicine \& Sciences, BHF Centre of Research Excellence James Black Centre, King's College London, 125 Coldharbour Lane, London SE5 9NU

Prof. Dr. mult. Thomas Meyer (second reviewer)

E-mail: $\quad$ thomas.meyer@med.uni-goettingen.de

Postal address: Department of Psychosomatic Medicine and Psychotherapy

University Medical Centre Göttingen, Waldweg 33, 37073 Göttingen

PD Dr. rer. nat. Laura Zelarayán-Behrend (second member of the thesis committee)

E-mail: $\quad$ laura.zelarayan@med.uni-goettingen.de

Postal address: Institute of Pharmacology and Toxicology, University Medical Centre Göttingen Georg-August-University Göttingen, Robert-Koch-Straße 40, 37075 Göttingen

Prof. Dr. rer. nat. Lutz Walter (third member of the thesis committee)

E-mail: $\quad$ walter@dpz.eu

Postal address: Department of Primate Genetic, German Primate Center Kellnerweg 4, 37077 Göttingen

PD Dr. rer. nat. Sven Thoms

E-mail: $\quad$ sven.thoms@med.uni-goettingen.de

Postal address: Department of Child and Adolescent Health, University Medical Centre Göttingen Georg-August-University Göttingen, Robert-Koch-Straße 40, 37075 Göttingen

Prof. Dr. Rüdiger Behr (external independent examiner appointed by King's College London)

E-mail: $\quad$ RBehr@dpz.eu

Postal address: Department of Degenerative Diseases, German Primate Center Kellnerweg 4, 37077 Göttingen 


\section{Table of contents}

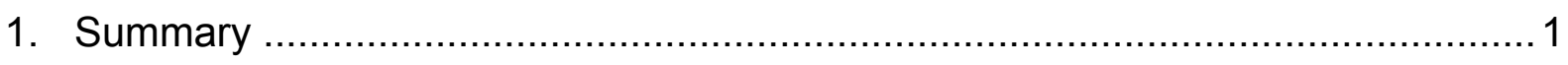

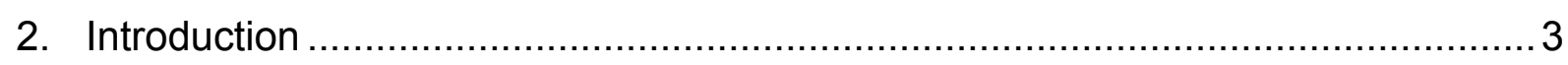

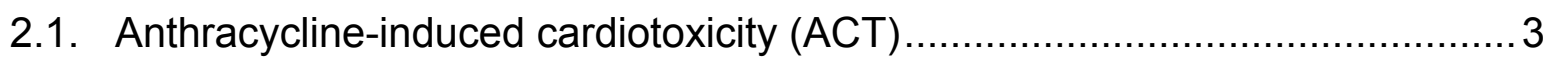

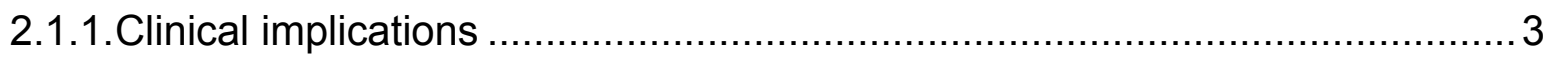

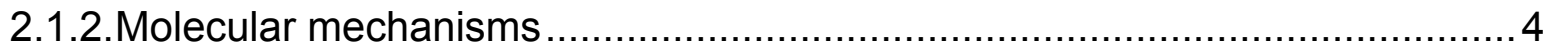

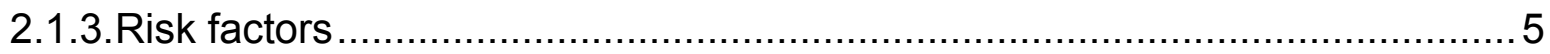

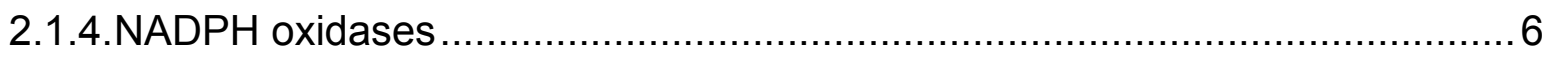

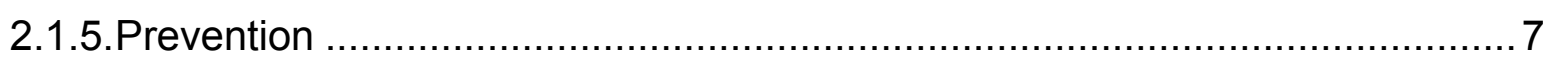

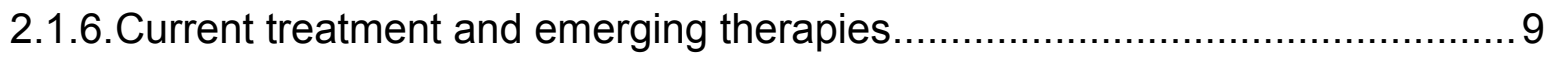

2.1.7. Telomerase activation as ACT-specific treatment .................................... 10

2.2. Disease modeling with induced pluripotent stem cells ............................. 12

2.2.1.iPSC lines from cancer patients with and without ACT ............................. 13

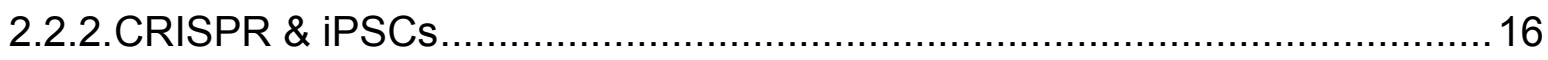

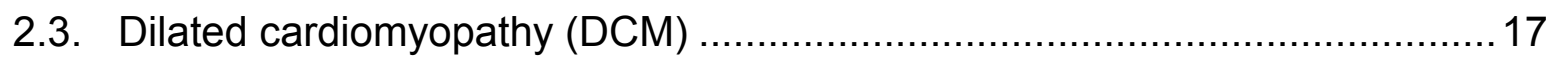

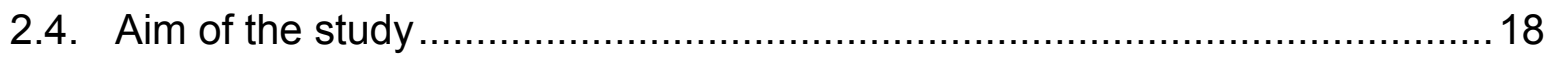

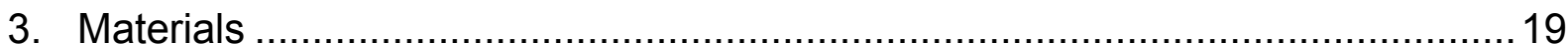

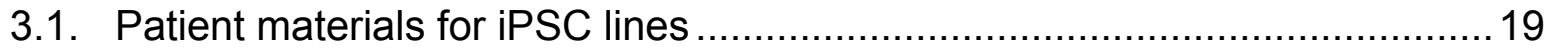

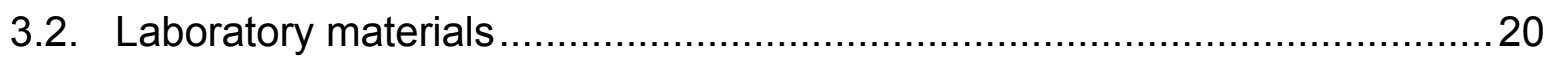

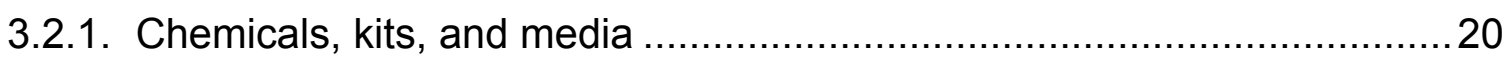

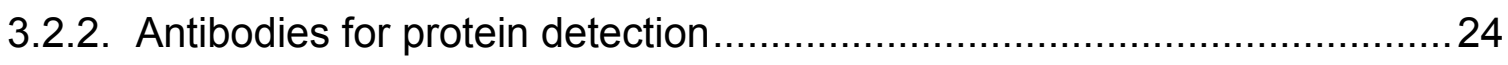

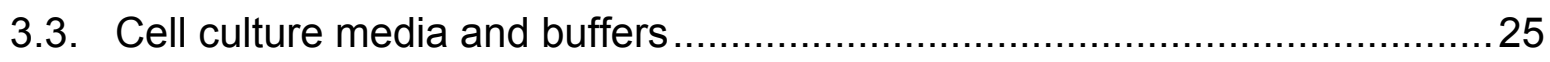

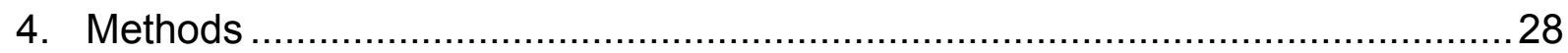

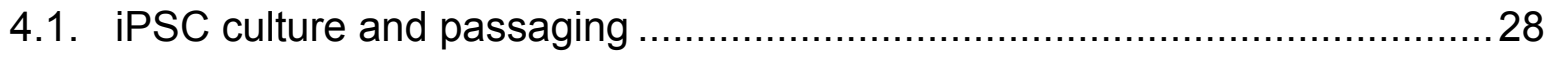

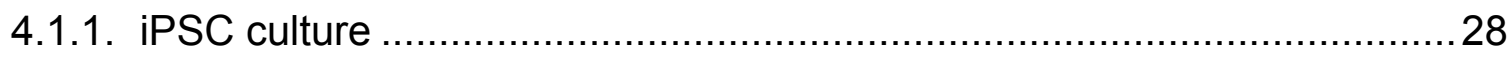

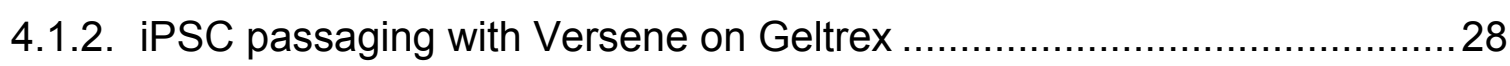

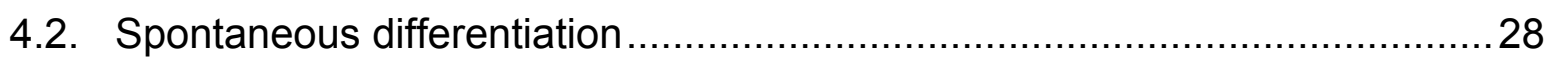

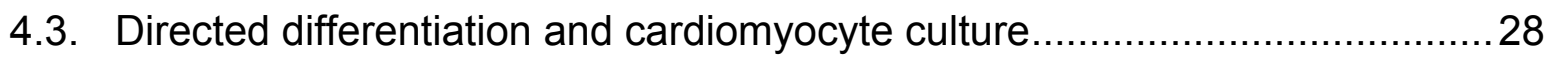

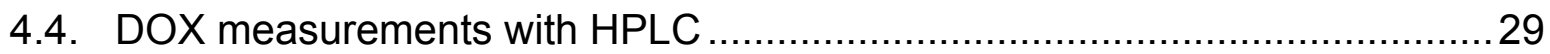

4.4.1. Sample preparation for intracellular DOX measurement .......................29

4.4.2. Subcellular fractionation for DOX detection .........................................29

4.4.3. HPLC measurement and data processing .......................................... 30

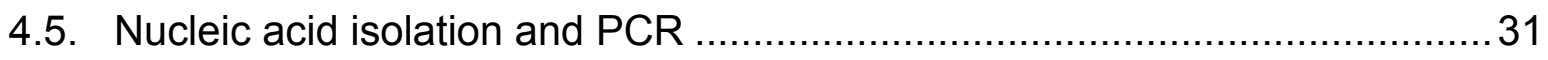




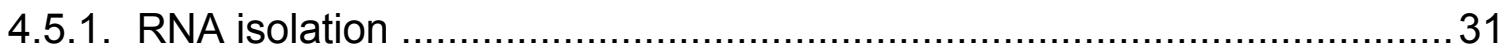

4.5.2. DNA isolation with QIAamp DNA Mini Kit ........................................... 31

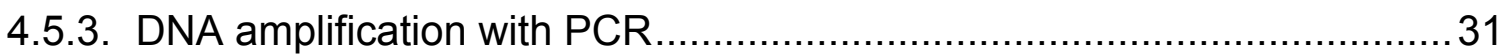

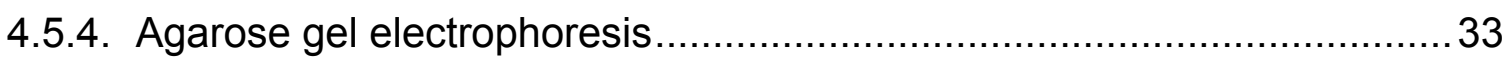

4.6. mRNA translation assay with puromycin and Western blotting .....................33

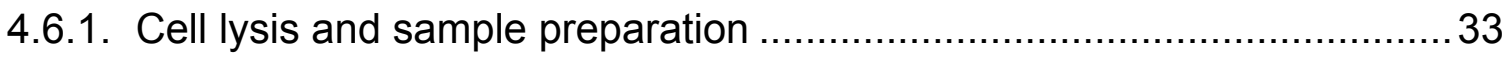

4.6.2. SDS-gel electrophoresis and Western blot ........................................... 34

4.6.3. Protein detection with specific antibodies ............................................ 34

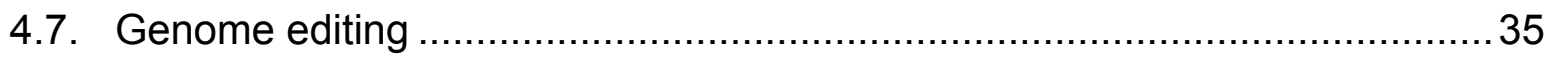

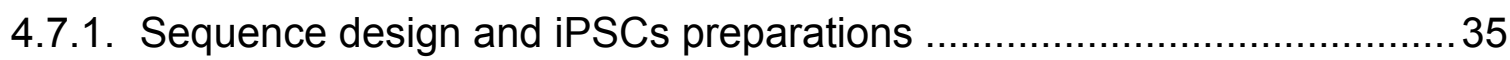

4.7.2. Complex formation and transfection preparation .................................. 35

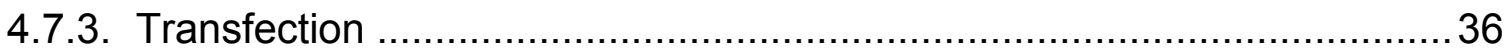

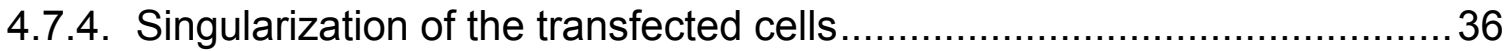

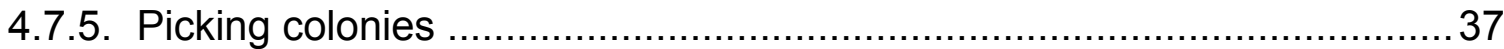

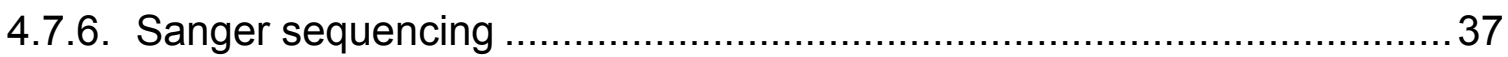

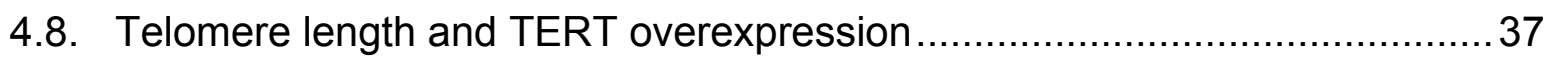

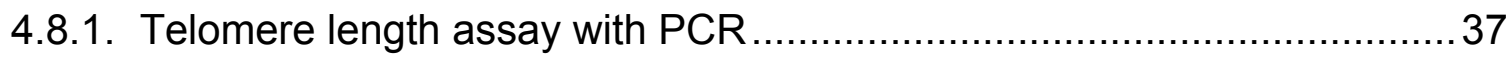

4.8.2. Telomere length assay with quantitative fluorescent in situ hybridization 37

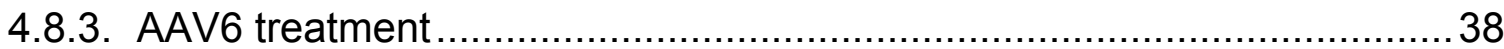

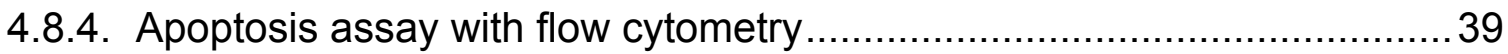

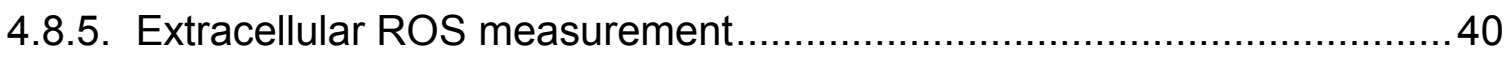

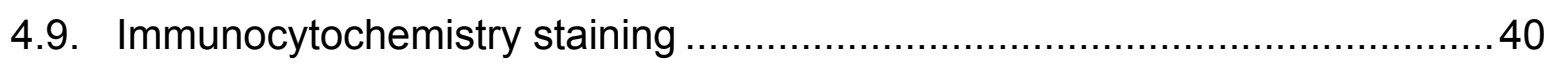

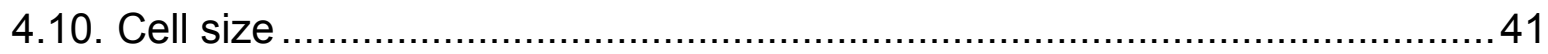

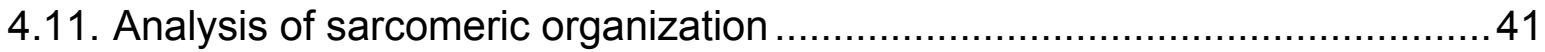

4.12. Statistical analysis ............................................................................... 42

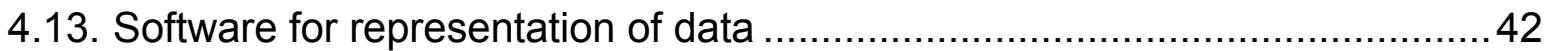

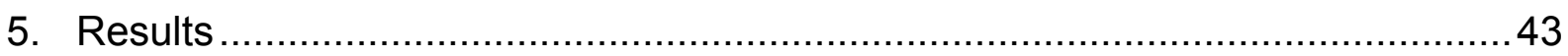

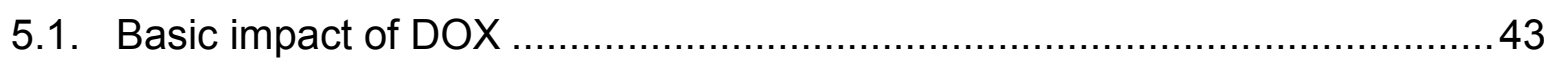

5.1.1. Resorption and retention of DOX in an ACT model ..............................4 43

5.2. Effects of DOX treatment on mRNA translation ......................................50

5.3. Correcting SNPs with CRISPR/Cas9 gene editing ...................................52

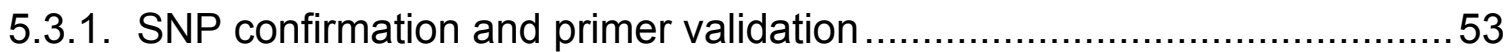

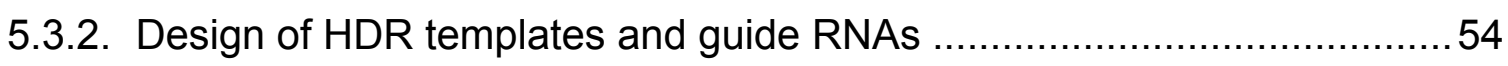

5.3.3. iPSC electroporation and culture with HDR enhancer .............................55 


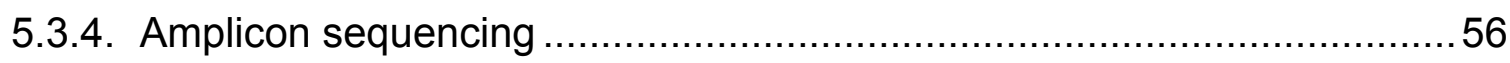

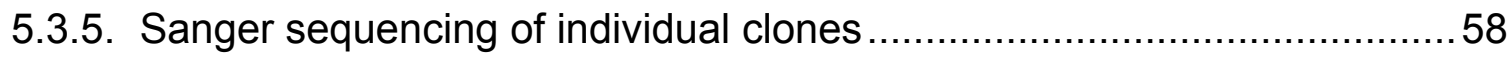

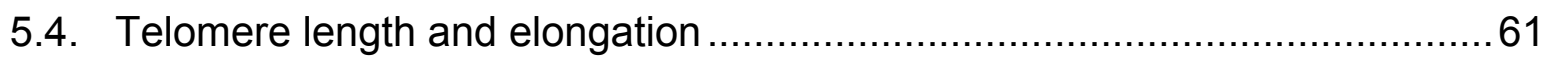

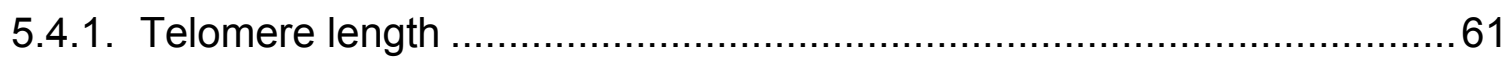

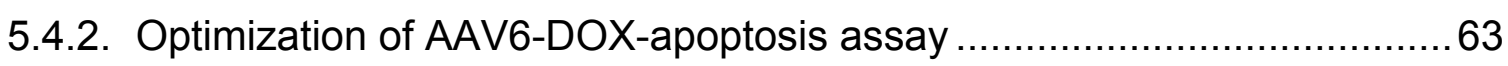

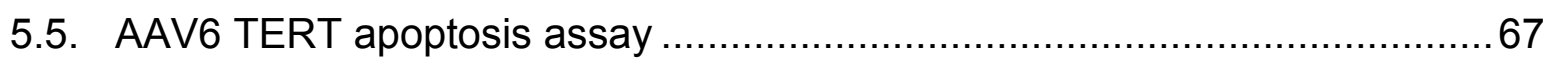

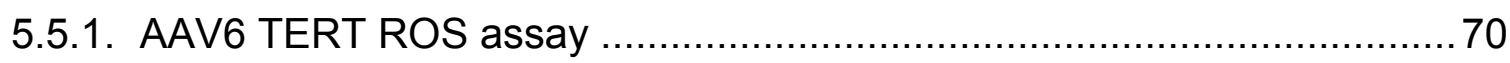

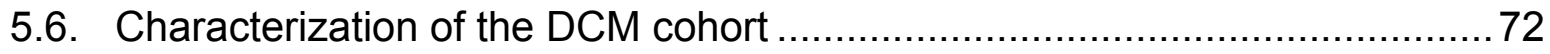

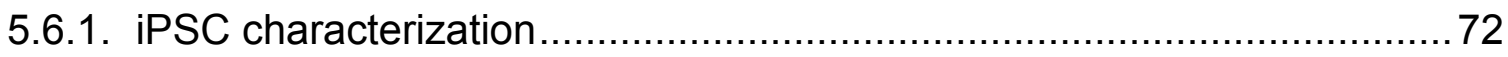

5.6.2. Generation and characterization of iPSC-derived cardiomyocytes ...........76

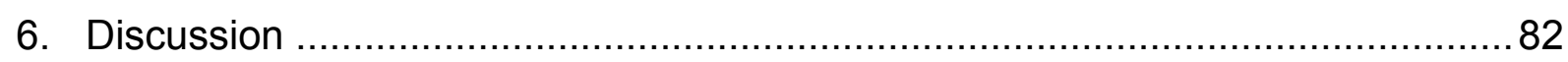

6.1. Uptake, retention, and distribution of DOX in iPSC-CMs …......................82

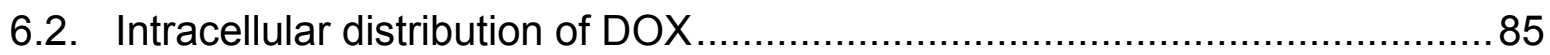

6.3. DOX-induced translational decrease in iPSC-CMs ................................... 87

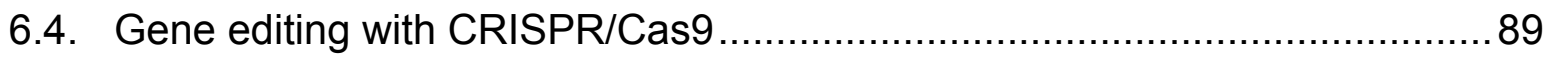

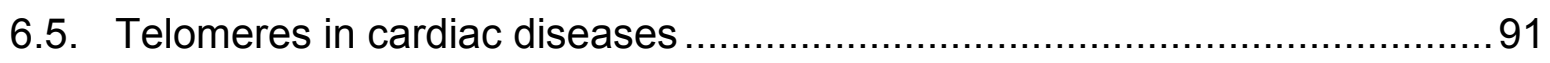

6.6. Overexpression of TERT with AAV vectors .............................................92

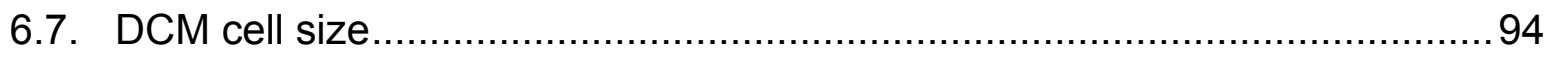

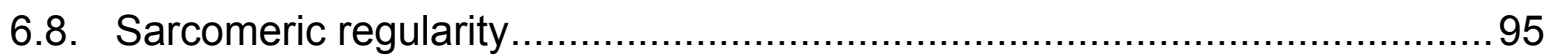

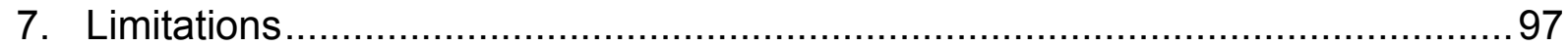

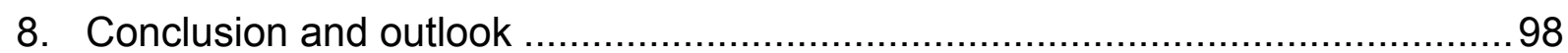

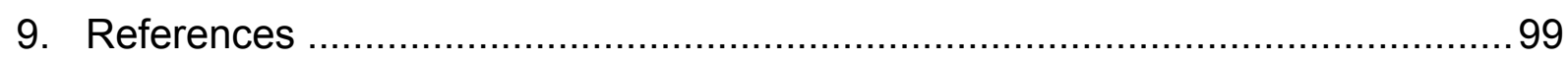

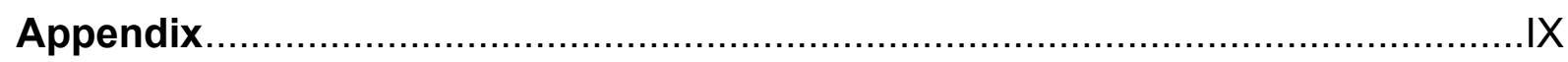

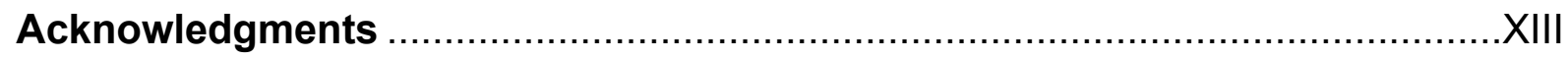

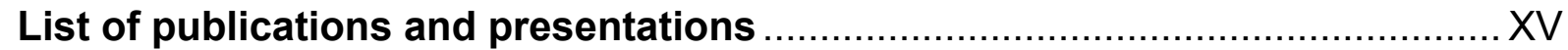

Curriculum vitae .............................................. Fehler! Textmarke nicht definiert. 


\section{List of figures}

Figure 2-1: Schematic overview of telomere length and cardiovascular diseases. 12

Figure 2-2: Scheme of the creation and use of patient-specific iPSC-CMs in ACT disease modelling. 15

Figure 2-3: Strategy to generate isogenic iPSC controls that differ at risk sites. 17

Figure 4-1: Schematic overview of the membrane isolation workflow. 30

Figure 4-2: Circular map of the AAV6 hTERT vector. 38

Figure 4-3: Circular map of AAV6 empty vector. 39

Figure 5-1: Intracellular DOX levels in iPSC-CMs increased with rising DOX concentrations.

Figure 5-2: Intracellular DOX levels in iPSC-CMs rose with increasing DOX treatment time.45

Figure 5-3: Decrease of intracellular DOX in iPSC-CMs after recovery period. 46

Figure 5-4: Subcellular distribution of DOX in iPSC-CMs after treatment with $1 \mu \mathrm{M}$ DOX for $24 \mathrm{~h}$. 48

Figure 5-5: DOX is distributed relatively evenly throughout fractions of DOX-treated iPSC-

CMs. 49

Figure 5-6: Global mRNA translation was decreased in iPSC-CMs after treatment with $1 \mu \mathrm{M}$ DOX for $24 \mathrm{~h}$. 51

Figure 5-7: Sanger sequencing results for SNP rs13058338. 53

Figure 5-8: Nucleotide sequences around the SNP with the gRNA binding sequence and the HDR template. 54

Figure 5-9: Time line of CRISPR/Cas9 gene editing with electroporation. 55

Figure 5-10: Representative bright field images of iPSCs one day after electroporation for CRISPR/Cas9 gene editing. 56

Figure 5-11: PCR product of RAC2 amplification 56

Figure 5-12: Screen shot from the BioEdit software. 59

Figure 5-13: Representative depiction of Sanger sequencing results. 61

Figure 5-14: Telomere lengths differed between iPSCs and iPSC-CMs. 62

Figure 5-15: qPCR results from telomere analysis. 63

Figure 5-16: MOI optimization for AAV6 infection of iPSC-CMs. 64

Figure 5-17: Bright field microscopy images of iPSC-CMs after AAV6 vector transduction._65

Figure 5-18: Apoptosis assay of DOX-treated, AAV6-transduced iPSC-CMs. 66

Figure 5-19: Flow cytometry apoptosis assay of iPSC-CMs after TERT overexpression and DOX treatment. 69

Figure 5-20: DOX treatment increased extracellular ROS in iPSC-CMs as measured with Amplex red. 
Figure 5-21: The generated iPSCs show pluripotency characteristics.

Figure 5-22: Expression of pluripotency genes in Crt-DCM and DCM lines on mRNA level. 74 Figure 5-23: PCR results show expression of germ layer marker proteins at three time points during spontaneous differentiation. 75

Figure 5-24: Immunofluorescence staining of alpha-actinin in iPSC-CMs. 76

Figure 5-25: iPSC-CM volume and iPSC-CM size measurements in 3D with a CASY cell counter. 77

Figure 5-26: Representative $\alpha$-actinin immunofluorescence images of DOX-treated iPSCCMs and untreated iPSC-CMs. 79

Figure 5-27: Integrity of the sarcomeric structure in iPSC-CMs of different donors. 81

\section{List of tables}

Table 2-1: Equivalent anthracycline dosages relative to doxorubicin. 8

Table 3-1: iPSC lines with respective reprogramming method and somatic material........... 20

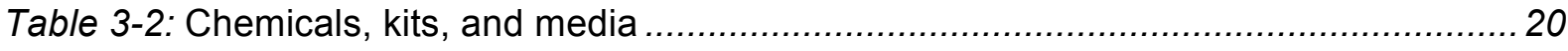

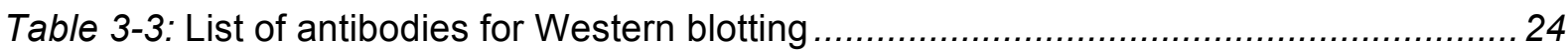

Table 3-4: List of antibodies for immunofluorescence staining. ........................................ 25

Table 4-1: Required components and their volume for PCR ......................................... 32

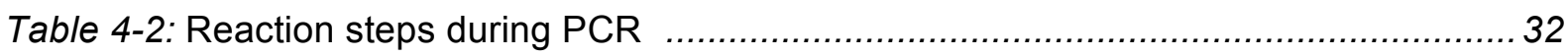

Table 4-3: Primer sequences for PCR primers. ........................................................... 32

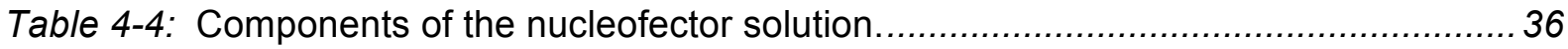

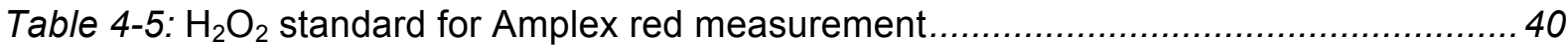

Table 5-1: Overview of the single-nucleotide polymorphism rs13058338 in the RAC2 gene. 53

Table 5-2: Amplicon sequencing results after CRISPR/Cas9 editing. ..............................57

Table 5-3: Editing efficiency according to BioEdit software. ......................................... 60

Table 5-4: Sanger sequencing of individual clones from CRISPR/Cas9-edited iPSCs. ........60 


\section{Abbreviations}

AAV

ABCC2

ACT

AMPK

ANOVA

ARB

$A U$

bp

BSA

CARP

CDNA

CHF

$\mathrm{CM}$

CMV

CRISPR

CrRNA

CTnT

DAPI

DCM

DEG

DHE

DMEM

DMSO

DNA

DOX

DSB

DTPA

DTT

EB

ECL

EDTA

EF

EGTA

EHM

ESC

FAD

FBS

FFTA

GAPDH

gDNA

GFP

gRNA

GSK3

$\mathrm{h}$

hbFGF

HCM
Adeno-associated virus

ATP binding cassettes subfamily $\mathrm{C}$ member 2

Anthracycline-induced cardiotoxicity

AMP-activated protein kinase

Analysis of variance

Angiotensin-receptor blockers

Arbitrary unit

Base pairs

Bovine serum albumin

Cardiac ankyrin repeat protein

Complementary DNA

Congestive heart failure

Cardiomyocyte

Cytomegalovirus

Clustered regularly interspaced short palindromic repeats

CRISPR RNA

Cardiac muscle troponin $T$

4.6-diamino-2-phenylindole

Dilated cardiomyopathy

Differentially regulated gene

Dihydroethidium

Dulbecco's modified Eagle medium

Dimethyl sulfoxide

Deoxyribonucleic acid

Doxorubicin

Double-strand break

Diethylenetriaminepentaacetic acid

Dithiothreitol

Embryoid body

Enhanced chemiluminescence

Ethylenediaminetetraacetic acid

Elongation factor

Ethylene glycol-bis(2-aminoethylether)-N,N,N',N'-tetraacetic acid

Engineered heart muscle

Embryonic stem cell

Flavin adenine dinucleotide

Fetal bovine serum

Fast Fourier transformation algorithm

Glyceraldehyde 3-phosphate dehydrogenase

Genomic DNA

Green fluorescent protein

Guide RNA

Glycogen synthase kinase 3

Hour

Recombinant human basic fibroblast growth factor

Hypertrophic cardiomyopathy 


\begin{tabular}{|c|c|}
\hline HDR & Homology-directed repair \\
\hline HEPES & 4-(2-Hydroxyethyl) piperazine-1-ethanesulfonic acid \\
\hline HPLC & High-performance liquid chromatography \\
\hline hTERT & Human telomerase reverse transcriptase \\
\hline IF & Immunofluorescence \\
\hline IMDM & Iscove's modified Dulbecco's medium \\
\hline iPSC & Induced pluripotent stem cell \\
\hline iPSC-CM & iPSC-derived cardiomyocyte \\
\hline ITR & Inverted terminal repeat \\
\hline IWP2 & Inhibitor of Wnt production 2 \\
\hline KLF4 & Krüppel-like factor 4 \\
\hline LIN28 & Lin-28 homolog A \\
\hline LVEF & Left ventricular ejection fraction \\
\hline MAPK & Mitogen-activated protein kinase \\
\hline MEF & Mouse embryonic fibroblasts \\
\hline MI & Myocardial infarction \\
\hline $\min$ & Minutes \\
\hline mito & Mitochondrial and nuclear fraction \\
\hline $\mathrm{MOI}$ & Multiplicity of infection \\
\hline mtDNA & Mitochondrial DNA \\
\hline MTG & Monothioglycerol \\
\hline mTOR & Mechanistic target of rapamycin \\
\hline MYBPC & Myosin binding protein $\mathrm{C}$ \\
\hline NADPH & Nicotinamide adenine dinucleotide phosphate \\
\hline NANOG & Homeobox protein NANOG \\
\hline NEAA & Non-essential amino acids \\
\hline NF-kB & Nuclear factor kappa-light-chain-enhancer of activated B cells \\
\hline Nox2 & NADPH-oxidase 2 \\
\hline Noxes & NADPH oxidases \\
\hline Oct & Octamer-binding transcription factor \\
\hline PAM & Protospacer adjacent motif \\
\hline PBMC & Peripheral blood mononuclear cell \\
\hline PBS & Dulbecco's phosphate-buffered saline \\
\hline PCR & Polymerase chain reaction \\
\hline $\mathrm{PI}$ & Propidium iodide \\
\hline PMSF & Phenylmethanesulfonyl fluoride \\
\hline qFISH & Quantitative fluorescent in situ hybridization \\
\hline qPCR & Quantitative polymerase chain reaction \\
\hline RNA & Ribonucleic acid \\
\hline ROS & Reactive oxygen species \\
\hline ROUT & Robust regression followed by outlier identification \\
\hline rpm & Revolutions per minute \\
\hline RPMI & Roswell Park Memorial Institute 1640 \\
\hline RT & Room temperature \\
\hline s & Seconds \\
\hline SDS & Sodium dodecyl sulfate \\
\hline SEM & Standard error of the mean \\
\hline sFB & Skin fibroblasts \\
\hline
\end{tabular}




$\begin{array}{ll}\text { SNP } & \text { Single-nucleotide polymorphism } \\ \text { SOX2 } & \text { Sex determining region Y-box 2 } \\ \text { SSEA-4 } & \text { Stage-specific embryonic antigen } 4 \\ \text { SUnSET } & \text { Surface sensing of translation } \\ \text { TBE } & \text { Tris-borat-EDTA } \\ \text { TBS } & \text { Tris-buffered saline } \\ \text { TBS-T } & \text { Tris-buffered saline-tween } \\ \text { TERT } & \text { Telomerase reverse transcriptase } \\ \text { TFA } & \text { Trifluoroacetic acid } \\ \text { TH } & \text { Tyrosine hydroxylase } \\ \text { TOP2 } & \text { Topoisomerase II } \\ \text { TZV } & \text { Thiazovivin } \\ \text { wt } & \text { Wild-type } \\ \beta-M E & \beta-M e r c a p t o e t h a n o l\end{array}$




\section{Summary}

Doxorubicin (DOX) has been used for decades to treat hematopoietic and solid tumors, although, in a subset of cancer survivors, it causes cardiotoxicity decades after treatment. The mechanisms of anthracycline-induced cardiotoxicity (ACT) are still incompletely understood. Induced pluripotent stem cells (iPSCs)-derived cardiomyocytes have become a valuable tool to study hereditary and structural cardiac conditions in vitro. A former study from our group showed that iPSC-derived cardiomyocytes (iPSC-CMs) from lymphoma patients with ACT were continuously more sensitive to DOX toxicity, demonstrating disorganized myofilament structure and altered calcium handling along with increased cell death and reactive oxygen species (ROS) production compared to control iPSC-CMs.

The aims of this study are to (1) analyze the cellular resorption and distribution of DOX, (2) assess its impact on mRNA translation, (3) address an ACT-associated single-nucleotide polymorphism (SNP) (rs13058338 in the RAC2 gene) in ACT disease progression, (4) test a potential treatment option with TERT overexpression, and finally, (5) distinguish between ACT and unspecific effects of DOX in human cardiomyocytes. To this end, iPSCs from ACTand dilated cardiomyopathy (DCM)-patients as well as control iPSC lines were differentiated into three-month-old iPSC-CMs and treated with DOX to model the effects of the drug exposure on human cardiomyocytes. In the first part of the study, the cellular resorption and retention of DOX in iPSC-CMs was investigated using high-performance liquid chromatography (HPLC) and positive correlations between DOX treatment time, treatment concentration and intracellular DOX levels were found. Intracellular DOX levels were consistently higher in ACT-iPSC-CMs compared to control iPSC-CMs and DOX remained longer in ACT-iPSC-CMs after treatment compared to control iPSC-CMs. In summary, these experiments suggested little regulation of DOX uptake or efflux in general. In the next chapter, the impact of DOX on mRNA translation was tested using the puromycin-protein incorporation assay. These data showed a significant decrease in translation down to $80 \%$ after DOX treatment in all tested iPSC-CMs as compared to untreated controls. In the third part of this work, the SNP rs13058338 was successfully edited in the RAC2 gene in an ACT patient IPSC line and heterozygous and homozygous isogenic gene-corrected ACT-iPSClines were established. The established, gene-edited iPSC lines will enable us to unravel the RAC2-SNP-related molecular contributions to ACT predisposition by testing the susceptibility to DOX-induced cardiotoxicity in isogenic ACT-iPSC-CMs. In order to analyze the contribution of cardiomyocyte-specific telomere lengths in ACT, quantitative polymerase chain reaction (qPCR) and quantitative fluorescent in situ hybridization (qFISH) experiments were performed. It was found that telomeres are shorter in iPSC-CMs compared to iPSCs and furthermore, that ACT-iPSC-CMs have shorter telomeres than their control. Telomerase 
(TERT) activity in cardioprotection was assessed with TERT overexpression in iPSC-CMs using adeno-associated-virus 6 (AAV6) vectors. We did not find measurable TERT-induced effects in the tested ACT-iPSC-CMs regarding apoptosis rates (Annexin V/PI kit for flow cytometry) or extracellular ROS production (Amplex red) after DOX treatment. Finally, DCMiPSC-CMs were included in the study to identify differences in disease mechanisms that are common in ACT-specific myocardial processes. Therefore, DOX-treated DCM-iPSC-CMs were tested for cell volume and sarcomeric regularity. DOX treatment had differential impacts on sarcomeric integrity of iPSC-CMs from the DCM cohort as compared to the ACT cohort, mainly due to highly deteriorated DCM-iPSC-CMs before DOX treatment. Control iPSC-CMs from the DCM cohort showed a similar decrease in sarcomeric integrity as control iPSC-CMs from the ACT cohort.

In conclusion, this thesis shows that ACT-iPSC-CMs can be used to determine subcellular DOX levels and, in addition, allows assessing the impact of this anthracycline on cellular processes such as mRNA translation. iPSC-CMs from ACT patients are more susceptible to the detrimental effects of DOX treatment than iPSC-CMs from controls or other cardiac disease such as DCM. This tool will allow for identifying the underlying genetic basis and mechanisms of ACT and may enable screening for protective agents. 


\section{Introduction}

\subsection{Anthracycline-induced cardiotoxicity (ACT)}

2.1.1. Clinical implications

The anthracycline drug doxorubicin (DOX) was first isolated in 1960 and tested in anti-cancer treatment soon afterward (Camerino and Palamidessi, 1960; Tan et al., 1967; Arcamone et al., 1969). Anthracycline-induced cardiotoxicity (ACT) was first described in 1971 (Middleman et al., 1971). Although it has now been known for decades that DOX can trigger cardiotoxicity, its outstanding efficacy against a broad range of solid and hematopoietic cancers makes it difficult to abandon its use. Currently, it is administered to $32 \%$ of breast cancer patients (Giordano et al., 2012), $57-70 \%$ of elderly lymphoma patients (Nabhan et al., 2015; Chihara et al., 2016), and almost $60 \%$ of pediatric cancer patients (van Dalen et al., 2014). In children younger than three years of age, the drug clearance is lower, which should be considered when calculating treatment plans (Völler et al., 2015). Clinical symptoms of ACT can occur acutely with DOX treatment or after treatment with refractory periods of several years. Diagnosis of ACT is based on arrhythmia, aberrant beating frequencies of the heart, decrease in left ventricular ejection fraction (LVEF), and fractional shortening (Magdy et al., 2016).

After it became evident that the risk of ACT rises with increasing doses of DOX, the life-long cumulative dose of DOX was limited to $500 \mathrm{mg} / \mathrm{m}^{2}$ (Lefrak et al., 1973; von Hoff et al., 1979; Swain et al., 1997). However, a decline in LVEF has been described with cumulative doses of as little as $150 \mathrm{mg} / \mathrm{m}^{2}$ (Plana et al., 2014; Henriksen, 2018). More recent studies suggest that the incidence of ACT is still around $5-9 \%$, with up to $18 \%$ of DOX-treated patients showing subclinical symptoms (Swain et al., 2003; Lotrionte et al., 2013; Cardinale et al., 2015).

ACT has been distinguished into two categories: an acute, reversible form with no morphologic changes in cardiac tissue, and a chronic, irreversible form, which shows myofibril loss and swelling of mitochondria, among other pathologies (Minotti et al., 2004). Recent, prospective studies by the Cardinale and Groarke labs are changing our understanding of ACT (Cardinale et al., 2015; Groarke and Nohria, 2015) as they suggested a single progressing disease. It has been reported that acute ACT occurs only during or within hours of DOX application, while the chronic form surfaces years or even decades after treatment (Steinherz, 1991). With the latest study results, it is conceivable that "chronic" cases could be detected shortly after treatment by assessing subclinical symptoms (Chung and Youn, 2016). Early diagnosis of ACT paired with appropriate treatment might lower the incidence of irreversible congestive heart failure (CHF) associated with the administration of this DNA-intercalating drug. 


\subsubsection{Molecular mechanisms}

DOX is applied in cancer treatment because it reliably arrests cancer cell growth and leads to apoptosis (recently reviewed by Cai et al., 2019). The exact mechanisms of the antineoplastic effects of DOX are still unclear; however, DOX is known to intercalate with DNA, produce double-strand breaks (DSBs), damage mitochondria, and induce elevated levels of reactive oxygen species (ROS) (Minotti et al., 2004). It is assumed that the properties that make DOX an effective anti-cancer drug are also responsible for its adverse effects on the heart.

\subsubsection{Elevated ROS levels}

DOX-induced damage through elevated ROS levels has been established as a key driver of ACT development for several decades (Doroshow et al., 1980; Nozaki et al., 2004; Ichihara et al., 2007). ROS-producing DOX-metal complexes were already described in the 1980s (Demant and Jensen, 1983). The so-called redox cycling is catalyzed by flavoproteins and produces superoxide (Doroshow, 1983; Davies and Doroshow, 1986; Hrdina et al., 2000). If the DOX complex is formed with $\mathrm{Fe}(\mathrm{III})$, nicotinamide adenine dinucleotide phosphate (NADPH) cytochrome P450 reductases and thiols facilitate the reduction to $\mathrm{Fe}$ (II) under production of ROS (Xu et al., 2005). However, ROS-producing redox cycling might not even be the most damaging result of the interaction between DOX and iron. DOX also inactivates iron-regulatory proteins (Ichikawa et al., 2014), leading to disturbed iron homeostasis and excessive iron accumulation in cardiomyocytes (CMs) (Ghigo et al., 2016). Even if iron is not directly part of the complex, it can catalyze reactions like Fenton reactions or Haber-Weiss reactions, which result in highly reactive hydroxyl radicals (Kaiserová et al., 2007).

Today it is known that small amounts of ROS are not merely a side product of cellular processes, but act in physiological and pathological cell signaling (Hancock et al., 2001; Dworakowski et al., 2006; Brieger et al., 2012; Hafstad et al., 2013). Elevated ROS levels, when not confined to specific cell compartments, lead to oxidative stress which in turn can cause adverse effects including DNA damage, senescence, and cell death (Cappetta et al., 2017).

\subsubsection{Mitochondrial damage}

The most damaging effect of DOX on the heart is likely its detrimental effects on mitochondria (Lebrecht and Walker, 2007). In CMs there are mitochondria high densities and are crucial for sustained production of ATP to enable contraction. DOX binds to cardiolipin in the inner mitochondrial membrane, which results in high DOX concentrations in the mitochondrial vicinity (Goormaghtigh and Ruysschaert, 1984; Nicolay et al., 1984; Goormaghtigh et al., 1990; Aryal and Rao, 2016). It also intercalates with mitochondrial DNA (mtDNA), which can have long-lasting deleterious consequences (Serrano et al., 1999). DOX 
interferes with the electron transport chain, disrupts mitochondrial membranes, and therefore induces mitochondrial ROS production (Gosalvez et al., 1974; Ichikawa et al., 2014).

\subsubsection{DNA damage}

DOX damages DNA in two ways. The drug intercalates directly into DNA by hydrogen bonding its amino group with cytosines (Agudelo et al., 2014). In addition, DOX binds to topoisomerase II (TOP2) and forms a TOP2-DOX-DNA complex (Lyu et al., 2007). TOP2 is a highly conserved enzyme, which introduces double-strand breaks to relax supercoiled DNA. There are two isoenzymes: TOP $2 \alpha$ and TOP $2 \beta$. DOX forms complexes with both isoforms, where it stabilizes an intermediate reaction complex, in which the cleaved DNA cannot be resealed (Kellner et al., 2002), which leads to increased cellular stress, growth arrest, and apoptosis (Tewey et al., 1984; Hong et al., 1990; Chen et al., 2012c). TOP2a is highly expressed in proliferating cells and is crucial for chromosomal segregation, which makes it a worthwhile target in anti-cancer therapy (Azarova et al., 2007). TOP2 $\beta$ is mainly present in quiescent cells, like cardiomyocytes, where it is constitutively expressed (Tewey et al., 1984; Zhang et al., 2012). TOP2 $\beta$ knockout mice were partially protected from DOX-induced cardiotoxicity, highlighting the importance of this process in the development of ACT and making it a potential target for therapy (Zhang et al., 2013; Vejpongsa and Yeh, 2014).

\subsubsection{Protein degradation}

DOX reversibly binds to proteasomes and serves as an allosteric, non-competitive inhibitor, leading to the accumulation of ubiquitinated proteins (Minotti et al., 2004). DOX induces autophagy, but it also blocks the completion of the process causing an accumulation of autophagosomes ( $\mathrm{Li}$ et al., 2016; Koleini and Kardami, 2017). These findings indicate that DOX severely limits cellular degradation and recycling processes. Taken together, there are multiple ways in which DOX inflicts oxidative stress, interrupts physiological rates of degradation, and causes cellular damage.

\subsubsection{Risk factors}

In order to prevent ACT, clinical studies have sought to find risk factors that can predict susceptibility to the development of the disease (Wojnowski et al., 2005; Reichwagen et al., 2015; Leong et al., 2017). Among accepted risk factors are previous cardiac diseases including hypertension and coronary artery disease (Qin et al., 2015), African-American ethnicity, and very young or old age (Lipshultz et al., 1995; Lotrionte et al., 2013; Wang et al., 2018). Female gender is associated with higher risk in pediatric cancer patients (Silber et al., 1993; Lipshultz et al., 1995; Meiners et al., 2018). In adult patients, it is unclear whether gender influences the risk (Meiners et al., 2018). Other risk factors include positive epidermal growth factor receptor 2 status or type II diabetes, where the latter was identified as risk factor for late-onset ACT (Qin et al., 2015). However, the high interindividual variability in the development of ACT does not allow for making profound predictions (Moazeni et al., 2017). 
Some patients tolerate high amounts of DOX, whereas others without any previous risk factors suffer from ACT after comparatively low doses. Recent clinical studies may narrow down the list of potential genetic causes (Blanco et al., 2012; Visscher et al., 2015; Vulsteke et al., 2015). However, they cannot reveal underlying mechanisms and, hence, cannot prove the involvement of the identified genes. It is of utmost importance to identify the involved mechanism, so the detrimental effects of DOX can be exploited for cancer treatment while other organs like the heart are protected.

\subsubsection{NADPH oxidases}

Among the genetic factors that were associated with the occurrence of ACT are singlenucleotide polymorphisms (SNPs) in genes encoding for subunits of the NADPH oxidase 2 (Nox2) (Wojnowski et al., 2005; Reichwagen et al., 2015; Vulsteke et al., 2015; Leong et al., 2017). The family of NADPH oxidases (Noxes) was first studied in neutrophils, where the enzyme complex is involved in the respiratory burst of phagocytes (Rossi and Zatti, 1964). It was the first time that a protein complex was identified, whose primary function was to generate ROS, rather than producing it as a side product. Noxes consist of six transmembrane domains and a cytoplasmic NADPH-binding site at the $\mathrm{COOH}$ terminus (Brandes et al., 2014a). In the proximity of the most COOH-terminal transmembrane domain is a flavin adenine dinucleotide (FAD)-binding region. Furthermore, each Nox complex has four highly conserved heme-binding histidines, two of which are located in the third and two others in the fifth transmembrane domain. In this way, Noxes can transport single electrons from NADPH via FAD, the first heme, and subsequently via the second heme to oxygen (Cave et al., 2006; Brandes et al., 2014b).

The family members Nox4, DUOX1, and DUOX2 produce $\mathrm{H}_{2} \mathrm{O}_{2}$, whereas Nox1, Nox2, Nox3, and Nox5 produce superoxide (Brandes et al., 2014a). Today, it is known that Nox expression and activity are not restricted to phagocytes. Nox1, Nox2, Nox4, and Nox5 are expressed in cardiac cells where they are involved in the regulation of gene expression, cell growth and senescence, differentiation and proliferation, as well as oxygen sensing (Petry et al., 2006; Bedard and Krause, 2007). In postmitotic tissues such as the heart, Nox-derived ROS can act as signaling molecules to induce regeneration after injury (Hervera et al., 2019). Nox2 and Nox4 are the predominately expressed Nox types in cardiomycytes and have been studied extensively (Ambasta et al., 2004; Pendyala and Natarajan, 2010; Zhang et al., 2013). While calcium activates Nox5, DUOX1, and DUOX2 (Dupuy et al., 1999; Kawahara et al., 2011), Nox4 is constitutively active and therefore regulated through expression (Martyn et al., 2006; Serrander et al., 2007). Nox4 is a membrane-bound protein complex, consisting of the catalytic subunit of Nox4 and p22phox. Nox1, Nox2, and Nox3 share some common cytosolic activators including the small GTPase Rac (Hancock et al., 2001; Bokoch and Diebold, 2002; Bokoch et al., 2009). Other activators and subunits are cell type specific 
(Brandes et al., 2014a). NOX2 gene expression is mediated through the transcription factors nuclear factor kappa-light-chain-enhancer of activated B cells (NF-KB) and activator protein 1 , which are, in turn, influenced by the redox state of the cell (Pendyala and Natarajan, 2010). G-protein-coupled receptor agonists, e.g., endothelin-1, angiotensin II, cytokines, and growth factors (Santos et al., 2011) activate Nox2. This process is tightly regulated and includes the Nox2 subunits p40phox, p47phox, and p67phox (Bedard and Krause, 2007; van der Vliet, 2008). The component p47phox is activated through multiple phosphorylations, which is initiated through mitogen-activated protein kinase 3 (MAPK 3), p38 MAPK, the serine/threonine kinase PAK1, and AKT signaling (El Benna et al., 1996; Groemping et al., 2003; Groemping and Rittinger, 2005; Raad et al., 2009). Nox2 activation is part of ROS signaling cascades, and as such, it is induced by tyrosine kinase receptors and MAPKs, as well as by ligands including tumor necrosis factor, angiotensin II, or epidermal growth factor (Holmström and Finkel, 2014; Park et al., 2016). Overactivation of Nox2 by DOX through aberrant signaling pathways increases intracellular ROS production and worsens oxidative stress (Angsutararux et al., 2015). SNPs that alter the function of this complex interplay might drive ACT development and cause a CHF phenotype. In CHF due to ACT, Nox2 is involved in apoptosis of CMs and cardiac fibrosis (Johar et al., 2006; Hayashi et al., 2008; Gilleron et al., 2009). In a mouse model, Nox2-derived ROS contributed to ACT (Zhao et al., 2010). The authors found contractile dysfunction, myocardial atrophy, apoptosis and interstitial fibrosis, which was also observed in vitro (Gilleron et al., 2009; Ma et al., 2013). Nox4 is a membrane-bound protein complex, consisting of the catalytic subunit of Nox4 and p22phox. While Nox2 activity is often associated with poorer outcome in cardiac conditions, the activity of Nox4 has been described as cardioprotective (Zhang et al., 2013).

\subsubsection{Prevention}

The risk factors mentioned above are not sufficient to develop safe individual DOX treatment plans for cancer patients. However, there have been other efforts to reduce the risk of cardiotoxicity. Some anthracyclines, including epirubicin, are less harmful than DOX but are usually administered with higher accumulative doses (Table 2-1). A meta-study of five randomized controlled trials, based on 1036 patients, found no benefit in the use of epirubicin regarding cardiotoxicity compared to DOX (van Dalen et al., 2010). Idarubicin, another anthracycline, is even more cytotoxic than DOX (Table 2-1). 
Table 2-1: Equivalent anthracycline dosages relative to doxorubicin.

\begin{tabular}{l|l|l} 
Anthracycline & Relative cardiotoxicity & Incidence of LVSD/CHF \\
\hline Doxorubicin & 1 & $3-5 \%$ at $400 \mathrm{mg} / \mathrm{m}^{2}$ \\
\hline Epirubicin & 0.7 & $0.9-11.4 \%$ at $900 \mathrm{mg} / \mathrm{m}^{2}$ \\
\hline Idarubicin & 0.53 & $5 \%$ at $150 \mathrm{mg} / \mathrm{m}^{2}$ \\
\hline Liposomal doxorubicin & 0.5 & $2 \%$ at $900 \mathrm{mg} / \mathrm{m}^{2}$
\end{tabular}

Data from McGowan et al., 2017.

A new generation of analogs of DOX has been synthesized, where a formamidine system replaced the amino group in daunosamine. Tested derivatives demonstrated better anticancer properties when compared with the conventional DOX and showed less cardiotoxicity in murine in vitro studies (Wasowska et al., 2005; Denel-Bobrowska and Marczak, 2017).

The manner of DOX administration affects the severity of side effects on the heart. Optimizing the drug administration can alleviate side effects without imparing anti-tumor efficacy. A clinical trial showed that prolonged infusion of DOX with reduced flow limits the peak plasma levels of DOX leading to reduced cellular drug uptake and a smaller risk of cardiotoxicity while maintaining efficacy against cancer cells (Legha et al., 1982). Mathematical models confirmed that prolonged infusion times are advantageous over bolus injection and liposomal delivery (El-Kareh and Secomb, 2000). In the meantime, novel developments, including galactosylated micelles and inducible liposomes, have improved the performance of enveloped drug delivery (Kneidl et al., 2014; Yu et al., 2014). A more recent meta-study concludes that in adults with a solid tumor, liposomal-encapsulated DOX is favorable over free DOX in terms of cardioprotection (van Dalen et al., 2014). Thermosensitive liposomes for DOX delivery increased DOX concentrations in the tumor vicinity, while reducing peak plasma concentrations (Abraham et al., 2005; Riccardi et al., 2013; Xing et al., 2015; Motamarry et al., 2017). In dogs and pigs, peak and overall drug concentrations in the cardiac muscle were $30-40 \%$ lower after liposomal DOX treatment compared to the free DOX (Kanter et al., 1993; Gyöngyösi et al., 2019). Porcine studies with Iyso-thermosensitive liposomal doxorubicin for the treatment of bladder cancer showed enhanced drug delivery to the target tissue and reduced burden on healthy tissues (Mikhail et al., 2017). Numerous phase 1 trials have shown that this approach is also applicable in a human setting. The clearance of liposomal DOX was more than five-fold lower, and the volume of distribution was approximately 10- to 15-fold lower compared to DOX alone (Swenson et al., 2003; Mross et al., 2004). Another single-center, phase 1 trial showed that ultrasound-triggered liposomes increase the intratumoral DOX concentrations on average by 3.7 times compared to standard DOX (Lyon et al., 2018). Although liposomal DOX did not protect from fibrosis induction, it attenuated ACT through the induction of interferon-related 
DNA damage resistance (Gyöngyösi et al., 2019). In summary, optimizing DOX administration through reduced flow rates, encapsulation, or through highly targeted delivery, lowers the risk of side effects on the heart.

To specifically reduce the risk of cardiac damage, DOX treatment has been complemented with classic cardioprotective drugs such as dexrazoxane or angiotensin-converting enzymeinhibitor, angiotensin-receptor blockers (ARB), and beta-blockers (Vejpongsa and Yeh, 2014). A randomized clinical study (NCT01375699) analyzed the effect of adjuvant treatment with the phosphodiesterase 5 inhibitor sildenafil and found no cardioprotection (Poklepovic et al., 2018). A recent meta-study suggested that the preventive use of $\beta$-blockers lowers the risk of ACT by mitigating the reduction in LVEF and possibly preserving the diastolic function (Shah et al., 2019). Barbosa and colleagues concluded in their meta-study that not all betablockers are suitable to avert ACT. While carvedilol and nebivolol showed a positive effect in clinical trials, the use of metoprolol showed no clear benefits (Barbosa et al., 2018). The authors did not specify which properties of the $\beta$-blockers were beneficial for ACT prevention. In addition, studies have found a number of antioxidants that could limit the ROS-induced side effects of DOX treatment (reviewed by Lindsey et al., 2014). Dexrazoxane has emerged as cardioprotective adjuvant for DOX treatment and has already been established in the clinic (Hasinoff et al., 2003; McMurray et al., 2014; Yancy et al., 2017). As an iron chelator, it has a completely different mode of action than conventional cardioprotective drugs (Kaiserová et al., 2007). Its cardioprotective properties are thought to stem from antioxidant properties that prevent the formation of highly reactive hydroxyl radicals (Hasinoff et al., 2003). A careful risk assessment based on the genetic background of the cancer patient, combined with new delivery paths and adjuvant cardioprotective treatment, will further limit the occurrence of ACT.

\subsubsection{Current treatment and emerging therapies}

Current treatment of ACT does not differ much from standard heart failure treatment. A more specific treatment regimen will be necessary to reduce the mortality rate and improve the standard of living of ACT patients. The guidelines published by the American College of Cardiology and the American Heart Association as well as the European Society of Cardiology already support the use of dexrazoxane as an adjuvant drug for ACT treatment resulting from DOX exposure (McMurray et al., 2012; Yancy et al., 2013, 2017). Furthermore, there is no ACT-specific treatment recommendation (Volkova and Russell, 2011; Zamorano et al., 2017). In a 2016 European Society of Cardiology position paper, the group recommended considering the lower limit of normal LVEF in echocardiography as $50 \%$ compared to LVEF before DOX treatment (Zamorano et al., 2017). If LVEF decreases to less than $50 \%$, the authors recommended ACE inhibitors (or ARBs), in combination with betablockers to prevent further LV dysfunction and progression to symptomatic CHF (Zamorano et al., 2017). With that recommendation, ACT treatment follows the standard heart failure 
treatment protocol (Yancy et al., 2013; Cardinale et al., 2015). Furthermore, the authors suggested the use of ACE inhibitors (or ARBs) and $\beta$-blockers in patients with symptomatic CHF or asymptomatic cardiac dysfunction (Zamorano et al., 2017). A retrospective study concluded that the use of $\beta$-blockers and renin-angiotensin inhibitors leads to LV functional recovery in ACT when applied within one year of cancer treatment (Ohtani et al., 2019). For late-onset $\mathrm{ACT}$, the antihypertensive drug sacubitril (neprilysin inhibitor), combined with the angiotensin receptor blocker valsartan, proved to be effective (Sheppard and Anwar, 2019). Randomized, prospective trials need to follow these findings to confirm the benefit for cancer patients.

Despite increasing awareness of ACT among oncologists as well as improved monitoring and treatment of cancer survivors, mortality due to cardiac conditions is an increasingly important factor of overall prognosis after cancer (McGowan et al., 2017). In a study of 64,000 female breast cancer patients, cardiovascular diseases were the leading cause of mortality surpassing recurrent breast cancer (15.9\% vs. $15.1 \%)$ after a median nine-year follow-up (Patnaik et al., 2011). Female breast cancer patients, who were cancer-free for nine years or more, were more likely to die of cardiovascular diseases than of recurrent breast cancer. Similarly, in survivors that were treated in their childhood and stayed cancerfree for 45 years, cardiac deaths accounted for $13 \%$ of all deaths (Mertens et al., 2008). Thus, with increasing cancer survivorship, cardiovascular mortality has already surpassed cancer mortality (McGowan et al., 2017) and is expected to continue to rise. Therefore, it is necessary to explore new treatment options directly aimed at ACT-specific disease mechanisms.

\subsubsection{Telomerase activation as ACT-specific treatment}

One of many treatment options for ACT-specific disease mechanisms is the activation of telomerase enzyme complex. The telomerase enzyme complex adds tandem TTG GGG repeats to existing terminal repeat sequences at the 3' end of DNA strands (Greider and Blackburn, 1985). Telomerase activity usually decreases with age, but reportedly increases in response to injury (Poss et al., 2002). Although telomerase expression is low in mammalian hearts, it exerts a pivotal function. In injured mouse hearts, telomerase expression increases not only in endothelial cells and fibroblasts but also in cardiomyocytes (Richardson et al., 2012). Telomerase reverse transcriptase (TERT) is the catalytic subunit of the telomerase enzyme comlex and the rate-limiting component for telomerase activity (Harrington et al., 1997; Kilian et al., 1997; Meyerson et al., 1997; Nakamura et al., 1997; Bodnar et al., 1998). The introduction of TERT into cultured cells made the cells overcome in vitro cellular senescence, which used to be inevitable as soon as they reached the Hayflick limit (Hayflick and Moorhead, 1961; Hayflick, 1965; Bodnar et al., 1998). Today, it is known that in many cancers, telomerase is reactivated and allows cancer cells to avoid senescence 
and apoptosis. Hence, telomere maintenance was named a key component for unlimited replication in cancer cells (Hanahan and Weinberg, 2000).

Most telomere research in the past decades has been carried out in the context of cancer and aging. However, more recently, the importance of telomere length and telomerase activity in cardiovascular diseases has become evident (Figure 2-1) (Yeh and Wang, 2016). Short telomeres have been identified as cornerstones of heritable cardiomyopathies (reviewed by Chang and Blau, 2018), and telomerase activation has been proposed for telomere-associated diseases and telomeropathies in the context of cardiovascular disease (Martínez and Blasco, 2018). Bär and Thum speculated that cancer patients would benefit from telomerase activation regarding cardiotoxicity with few disadvantages to anti-cancer treatment (Bär and Thum, 2017). High telomerase activity has been linked to low mitochondrial $\mathrm{H}_{2} \mathrm{O}_{2}$ production and cardioprotection in mice after myocardial infarction (MI) (Bär et al., 2014; Beyer et al., 2016; reviewed by Quryshi et al., 2018). Under oxidative stress, TERT is excluded from the nucleus and is relocated to mitochondria (Haendeler et al., 2004, 2009). There it decreases the production of mitochondrial ROS and, hence, limits the occurrence of more oxidative stress (Beyer et al., 2016). DOX treatment increases cellular ROS levels so that telomerase activation decreased the burden on cardiac cells. Telomerase activation also restored telomeres that were shortened by oxidative stress (von Zglinicki, 2002, 2006), and therefore increased cellular lifespan. The heart displays regenerative potential, however, the potential of CMs is limited (Rosenzweig, 2012). Elongation of telomeres and TERT activation is likely to extend cellular lifespan in CMs and would possibly improve the outcome in cancer patients that suffer from DOX-induced cardiotoxicity. 


\section{T T T T T A}
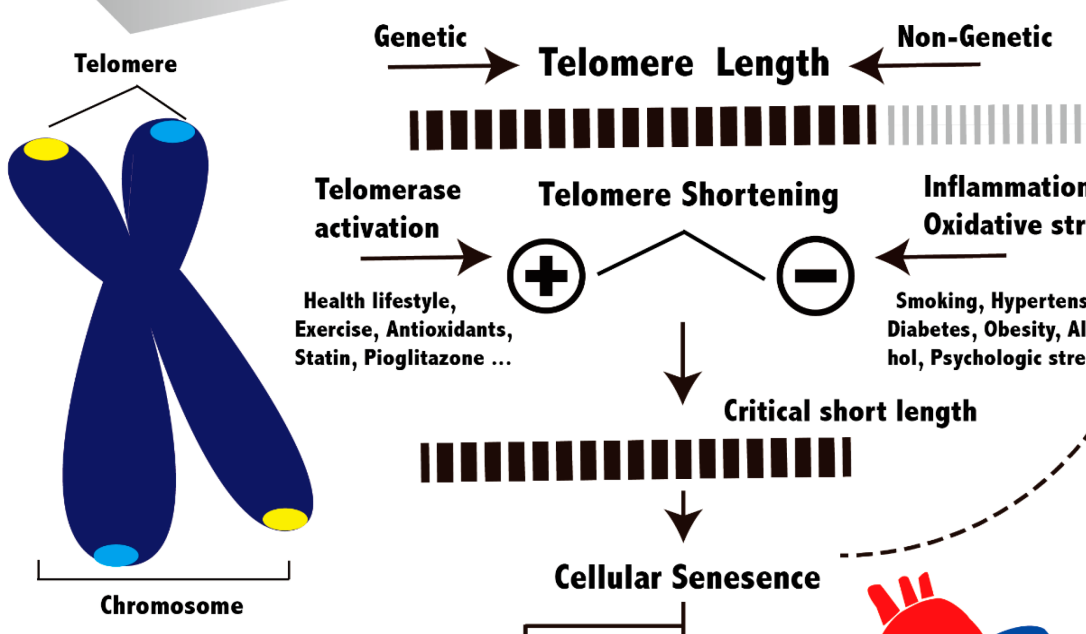

Telomere Shortening

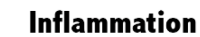

Exercise, Antioxidants,

tatin, Pioglitazone ...

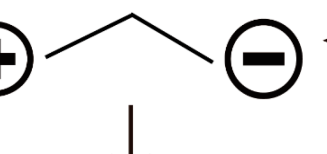

Oxidative stress

Smoking, Hypertension, I

Diabetes, Obesity, Alco- I

hol, Psychologic stress ..?

\section{|HIIIIIIIIIHII}

Critical short length
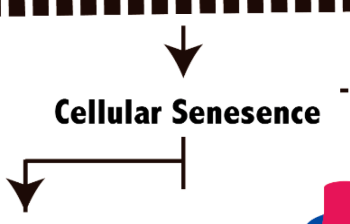

Atherosclerosis

Cardiovascular Diseases

Coronary artery disease
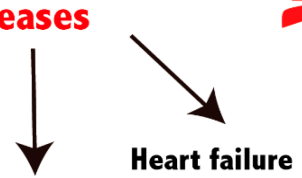

Stroke

Heart failure

Peripheral artery disease

Figure 2-1: Schematic overview of telomere length and cardiovascular diseases.

Telomere length is influenced by genetic and non-genetic factors. Once telomeres reach critically short lengths senescence is triggered and limits cellular function. On a larger scale, senescence of cardiac cells contributes to cardiovascular disease. From Yeh and Wang, 2016, shared under the creative commons attribution license.

\subsection{Disease modeling with induced pluripotent stem cells}

Pluripotent stem cells are defined by their ability to self-renew and to differentiate into cells of all three germ layers and the germline (Jaenisch and Young, 2008). In the past, most stem cell research was carried out with embryonic stem cells (ESCs), which entailed ethical concerns (King and Perrin, 2014; Ilic and Ogilvie, 2017). In 2006, Takahashi and Yamanaka reported the reprogramming of murine fibroblasts into cells with properties of pluripotent stem cells (Takahashi and Yamanaka, 2006). Retroviral transduction of four transcription factors (octamer-binding transcription factor [Oct]-3/4, sex determining region Y-box 2 [SOX2], C$M Y C$, and Krüppel-like factor 4 [KLF4]) was sufficient to yield pluripotent stem cells, which resembled ESCs in many criteria including self-renewal, morphology, expression of characteristic pluripotency genes, teratoma formation, and chimera development (Figure 2-2) (Takahashi and Yamanaka, 2006). Subsequently, it was shown that reprogramming of fibroblasts could also be done successfully with a different combination of transduced transcription factors (Yu et al., 2007), and it was even possible to reprogram human cells 
(Takahashi et al., 2007). For this groundbreaking work, Yamanaka was awarded the Nobel prize in Physiology or Medicine in 2012, which underlines the importance of this discovery. Soon after the initial reports, reprogramming had been done in a large set of cell types (Park et al., 2008). For differentiation into CMs with a high yield, Burridge and colleagues proposed a straightforward three-step protocol (Burridge et al., 2014), which modulated the canonical Wnt pathway. Effective modulation of this pathway through small molecules had been reported earlier (Gonzalez et al., 2011; Ren et al., 2011; Lian et al., 2013). In the proposed differentiation protocol, the Wnt pathway is activated through inhibition of glycogen synthase kinase 3 (GSK3), which steers induced pluripotent stem cells (iPSCs) into mesodermal differentiation. Subsequent inactivation of the pathway through inhibitor of Wnt production 2 (IWP2) guides the differentiation towards iPSC-derived CMs (iPSC-CMs). To be able to detect differences in cardiomyocyte-specific parameters, it is important to have a high purity in the produced iPSC-CMs. High purity can be achieved with metabolic selection, in which their ability to metabolize lactate is used to purify iPSC-CMs. Selection media is glucose depleted and supplemented with lactate, which offers sufficient energy for iPSC-CMs, but not for other cell types (Tohyama et al., 2013).

iPSC-CMs offer the possibility to model human cardiac diseases and study them in a defined in vitro system. Over the past years, several disease models have been established. For example, Maillet and colleagues showed that disruption of TOP2B, which is associated with ACT in mouse studies, significantly reduced the perceptibility of human iPSC-CMs to DOXinduced DNA double-strand breaks and cell death (Maillet et al., 2016). Our group and others have successfully modeled DOX-induced cardiotoxicity (Burridge et al., 2016; Haupt, 2018) and showed that iPSC-CMs from patients recapitulate the predilection towards ACT. More recently, the development of a different form of anti-cancer-therapy-induced cardiotoxicity was successfully modeled with patient-specific iPSC-CMs: trastuzumab-induced cardiotoxicity. The authors reported that iPSC-CMs from affected individuals were more susceptible to trastuzumab treatment compared to iPSC-CMs from healthy controls (Kitani et al., 2019). In contrast to iPSC-CMs treated with DOX, trastuzumab-treated iPSC-CMs did not show cell death or sarcomeric disorganization. However, contractile function and calciumhandling of iPSC-CMs were impaired, which was partially attributed to decreased mitochondrial activity and aberrant cellular metabolic pathways (Kitani et al., 2019).

\subsection{1. iPSC lines from cancer patients with and without $A C T$} In our group, an ACT study cohort of three ACT-iPSC lines and two matching control iPSC lines were generated and characterized (Haupt, 2018). Patient selection and generation of the iPSC lines have been described in detail (Haupt, 2018). Briefly, patients were selected from the RICOVER 60 trial (Pfreundschuh et al., 2008) according to the occurrence of ACT and specific genetic traits. All patients had received comparable accumulative doses of DOX 
(Figure 2-2). At the time of the sample collection, three patients had developed ACT, while the control patients had no cardiac disease (Figure 2-2).

Furthermore, the three ACT patients showed homozygous or heterozygous SNPs in the RAC2 gene that encodes for an activator of the NADPH oxidase. These SNPs have been associated with ACT development in clinical studies (Wojnowski et al., 2005; Reichwagen et al., 2015; Vulsteke et al., 2015; Leong et al., 2017). The two control patients did not show the respective SNPs. From each patient, two cell lines were characterized and differentiated into iPSC-CMs. Haupt showed that after DOX treatment, iPSC-CMs from ACT patients differed from control iPSC-CMs in calcium handling, sarcomeric regularity, ROS production, and apoptosis rates (Haupt, 2018). Overall, ACT was successfully modeled in vitro by showing that ACT-iPSC-CMs were affected more by DOX treatment than control iPSC-CMs. 


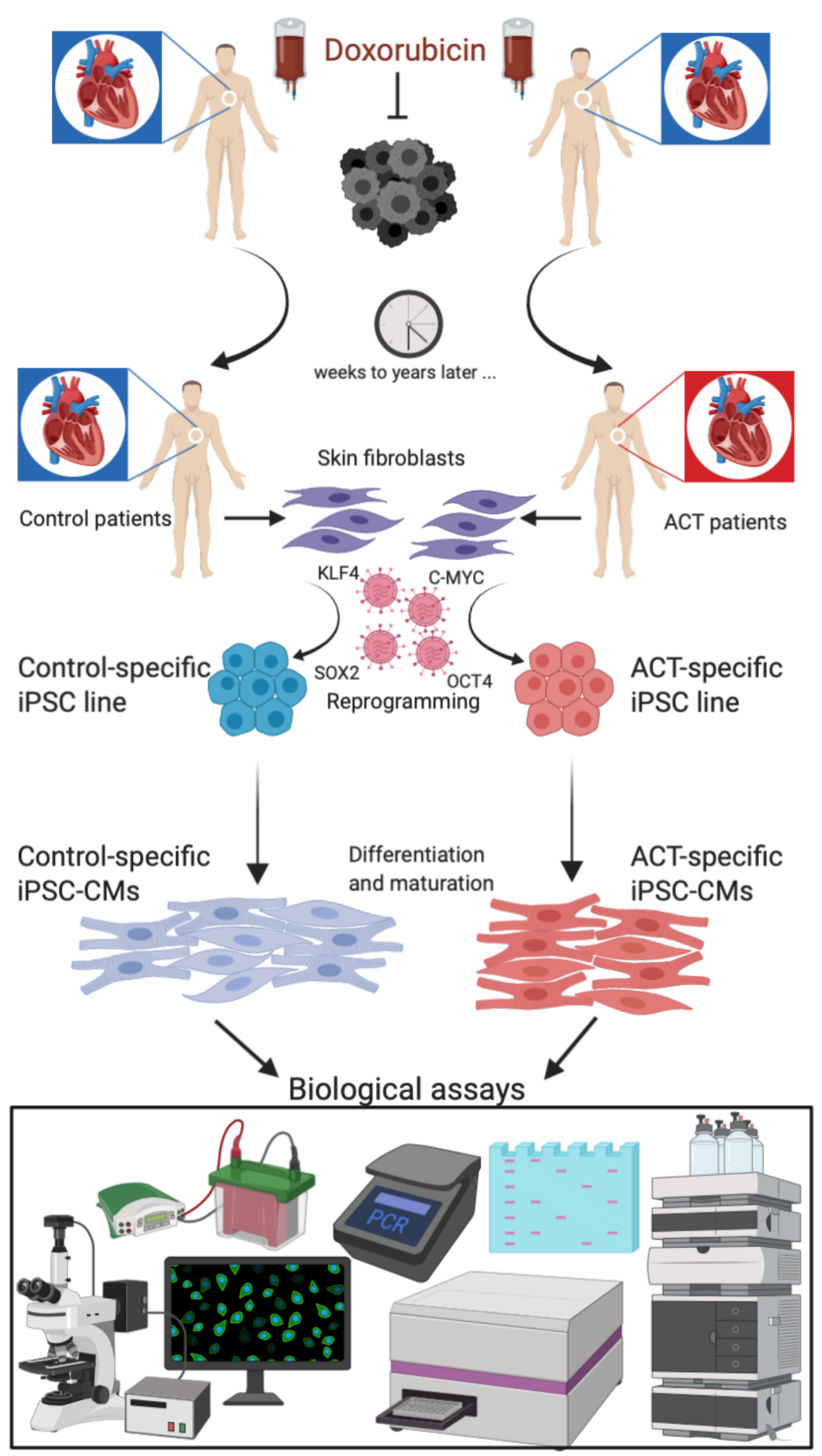

Figure 2-2: Scheme of the creation and use of patient-specific iPSC-CMs in ACT disease modelling. 
Cancer patients were treated with Doxorubicin. One part of them developed ACT over time, while the other part did not suffer from cardiac disease. Skin fibroblasts from ACT patients and control patients were reprogrammed into iPCs (induced pluripotent stem cells) using viral transduction of the Yamanka factors KLF4, C-MYC, SOX2, and OCT4. iPSCs were differentiated into iPSC-CMs (iPSCderived cardiomyocytes). The ACT patient specific- and control patient-specific iPSC-CMs were studied with different biological assays.

\subsubsection{CRISPR \& iPSCs}

The discovery of the clustered regularly interspaced short palindromic repeats (CRISPR) locus (Mojica et al., 1993) together with the identification of the CRISPR-associated 9 (Cas9) enzyme and the protospacer adjacent motif (PAM; Bolotin et al., 2005) paved the way for the development of a novel, simple, fast, and specific tool for genome editing. In 2012, two independent groups presented a CRISPR/Cas9 system, in which CRISPR RNA (crRNA) and trans-activating RNA are fused to one guide RNA (gRNA), which can theoretically be designed to target any DNA site by simply changing the sequence of the crRNA (Gasiunas et al., 2012; Jinek et al., 2012). Soon after that, the system was successfully adapted to eukaryotic cells (Cong et al., 2013). This adaption enabled researchers to develop in vivo repair approaches for genetic diseases and has, therefore, been praised as "resolving the taxonomy of human disease" (Merkle and Eggan, 2013). CRISPR/Cas9 also potentiated disease modeling with iPSCs (Hockemeyer and Jaenisch, 2016). A former obstacle of iPSC disease modeling was the creation of meaningful controls. With CRISPR/Cas9, it is possible to alter single or multiple risk alleles specifically. In this way, disease-associated mutations can be introduced in iPSCs from healthy individuals, or mutations in patient-iPSCs can be corrected (Figure 2-3). These isogenic controls, which only differ in the respective risk allele, allow for relevant comparisons and valid conclusions. In the field of cardiovascular research, many diseases with genetic causes have successfully been modeled with CRISPR/Cas9edited iPSCs (reviewed by Motta et al., 2017). The combination of CRISPR/Cas9 editing and iPSCs was pronounced as "a platform for personalized and precision cardiovascular medicine" (Matsa et al., 2016). For ACT modeling, the tool can facilitate the identification of genetic causes and risk factors. Most recently, CRISPR/Cas9-edited iPSC models were reported for Noonan syndrome (Higgins et al., 2019; Jaffré et al., 2019), effective truncations in titin and dystrophin (Pioner et al., 2019; Zaunbrecher et al., 2019), functional annotation of cTnT (cardiac muscle troponin T) (Lv et al., 2018), and short- and long QT syndrome (Guo et al., 2019; Wang et al., 2019). 
Control-specific iPSC line

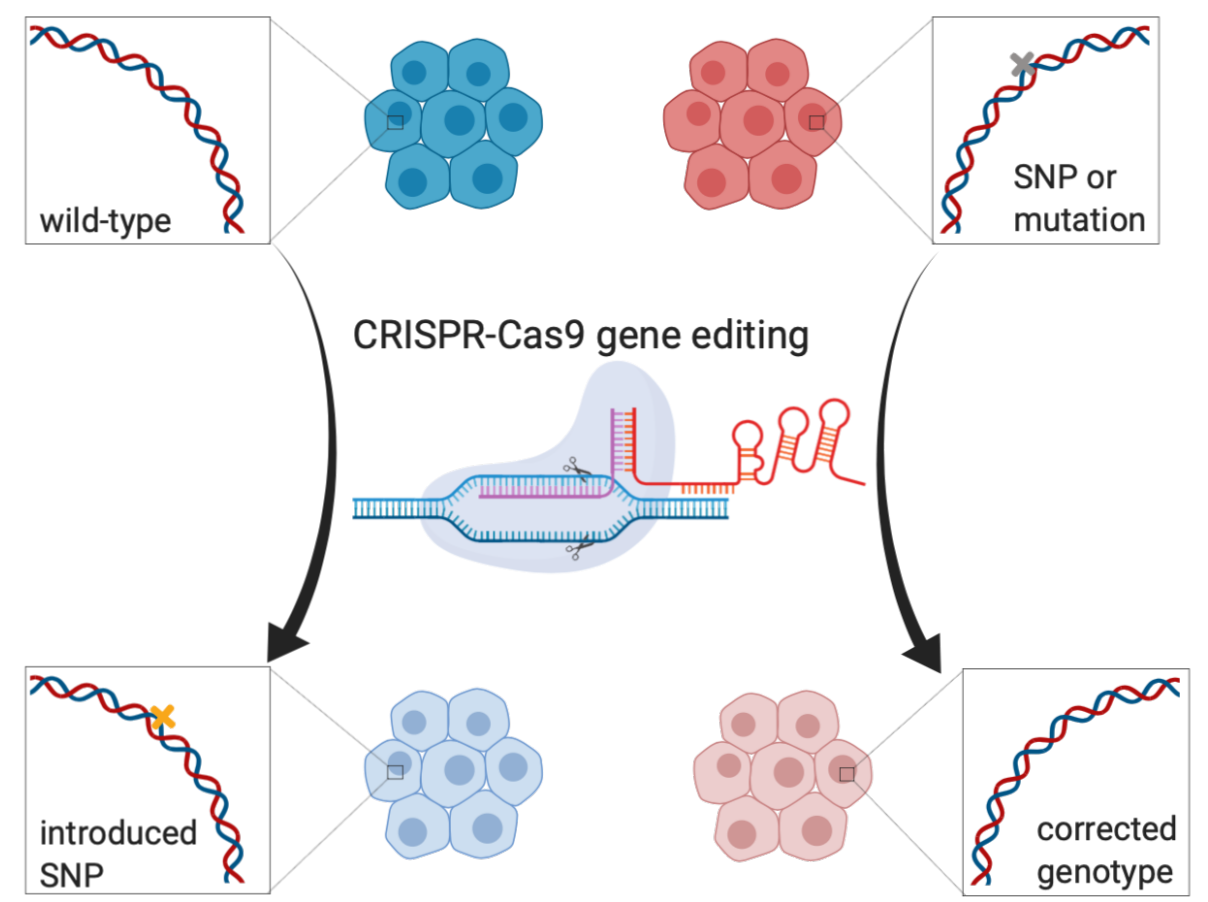

Figure 2-3: Strategy to generate isogenic iPSC controls that differ at risk sites.

With CRISPR gene editing, wild-type sequences of control iPSCs can be rendered disfunctional (left). SNPs in patient iPSC lines can be corrected into their functional wild-type form (right).

\subsection{Dilated cardiomyopathy (DCM)}

Dilated cardiomyopathy (DCM) is caused by a dilation of the left ventricular wall, which impairs the heart's ability to pump blood effectively (Dec and Fuster, 1994; Cabrera Cabrera, 1999). Hence, DCM is often accompanied by a reduction of LVEF and leads to heart failure symptoms like fatigue or shortness of breath. In most cases, the initial factor for the onset of the condition remains unknown, therefore, DCM is also known as 'idiopathic' cardiomyopathy (Elliott, 2000). DCM can have genetic causes, most commonly affecting the large sarcomeric protein titin (Kamisago et al., 2000; Gerull et al., 2002; Hinson et al., 2015). DCM can also be triggered by a variety of other factors, including previous cardiovascular disease (Khasnis et al., 2005), alcoholism, diabetes, thyroid disease, viral infections of the heart, heart valve abnormalities, childbirth (Patten et al., 2012), or drugs (such as doxorubicin) that trigger cardiotoxicity (reviewed by Japp et al., 2016). The diagnosis of DCM occurs after other cardiomyopathies such as hypertensive heart disease, myocardial non-compaction, and athlete's heart are excluded (reviewed by Japp et al., 2016). Especially in the case of anthracycline-induced DCM, it would be beneficial to detect disease onset before clinical sympthoms arise. That would offer the opportunity to adjust or abrogate anthracycline treatment and avoid further adverse effects on the heart. While electrocardiogram and echocardiography are suggested to monitor individuals with high-risk mutations (Mahon et 
al., 2005; Fatkin et al., 2011), myocardial strain imaging by echocardiography has proven to be a reliable method to detect early signs of ACT (reviewed by Thavendiranathan et al., 2014). In that case, a reduction of more than $10 \%$ in longitudinal strain is a clear indicator of developing ACT (reviewed by Japp et al., 2016). It is still unknown whether genetics, anthracyclines, or other causes drive disease onset in distinctly different ways, or whether there are common molecular processes that lead to a DCM phenotype. Our group and others have modeled inherited DCM with patient-specific iPSC-CMs (Sun et al., 2012; StreckfussBömeke et al., 2017). Together with iPSC lines from ACT patients, this approach has the potential to unravel differences and similarities in basic cardiomyopathy mechanisms.

\subsection{Aim of the study}

DOX treatment has multiple and profound effects on CM homeostasis. The aims of this study are:

- Analyze the resorption and intracellular distribution of DOX in iPSC-CMs from ACT patients and control patients.

- Identify the molecular effects of DOX on basic processes including mRNA translation in iPSC-CMs from ACT and control patients.

- Edit the ACT-associated SNP rs13058338 in NADPH-oxidase subunit RAC2 into wildtype with CRISPR/Cas9 gene editing.

- Explore the potential of adeno-associated virus 6 (AAV6)-mediated overexpression of TERT as ACT-specific treatment.

- Distinguish between ACT and DCM-specific effects of DOX in patient-specific iPSC-CMs. 


\section{Materials}

This section lists patient materials, consumables, chemicals, commercial kits, and assays, antibodies, buffers, solutions, and media that were used for this study.

\subsection{Patient materials for iPSC lines}

iPSC lines created from cardiomyopathy patients, cancer patients, and healthy controls were used in this study. Relevant background information about the donors, their disease status, and the type of somatic material are listed below.

- ACT patient 1: Derived from skin fibroblasts (sFB) of a 69-year old male B-cell lymphoma patient with ACT (309 $\mathrm{mg} / \mathrm{m}^{2}$ median DOX treatment).

- ACT patient 2: Derived from sFB of a 71-year old male B-cell lymphoma patient with ACT (309 $\mathrm{mg} / \mathrm{m}^{2}$ median DOX treatment).

- ACT patient 3: Derived from sFB of a 66-year old female B-cell lymphoma patient with ACT (309 $\mathrm{mg} / \mathrm{m}^{2}$ median DOX treatment).

- Control patient 1: Derived from sFB of a 66-year old male B-cell lymphoma patient without cardiac complaints following anthracycline chemotherapy $\left(318 \mathrm{mg} / \mathrm{m}^{2}\right.$ median DOX treatment).

- Control patient 2: Derived from sFB of a 68-year old male B-cell lymphoma patient without cardiac complaints following anthracycline chemotherapy $\left(318 \mathrm{mg} / \mathrm{m}^{2}\right.$ median DOX treatment).

- DCM 1: Derived from sFB of a 44-year-old male patient with a sudden dilative cardiomyopathy from the Department of Cardiology and Pneumology, University Medical Center Göttingen.

- DCM 3: Derived from peripheral blood mononuclear cells (PBMCs).

- Ctr-DCM 2: Derived from PBMCs of a non-consanguineous family member of DCM 1 with no recorded history of cardiovascular disease from the Department of Cardiology and Pneumology, University Medical Center Göttingen.

- CRT1: Derived from PBMCs of a healthy, 52-year-old female individual with no recorded history of cardiovascular disease. Department of Cardiology and Pneumology, University Medical Center Göttingen.

All procedures were conducted in compliance with the Institutional Ethical Committee, University Medical Center Göttingen. Written informed consent was received from the participants before inclusion. The study conforms to the World Medical Association declaration of Helsinki. An overview of the iPSC lines and their respective method of reprogramming are listed in Table 3-1. 
Table 3-1: iPSC lines with respective reprogramming method and somatic material.

\begin{tabular}{|c|c|c|c|c|}
\hline Cell line & $\begin{array}{l}\text { Internal } \\
\text { label }\end{array}$ & $\begin{array}{l}\text { Reprogramming } \\
\text { method }\end{array}$ & $\begin{array}{l}\text { Somatic } \\
\text { material }\end{array}$ & Published in \\
\hline ACT patient 1.1 & ACT 1.9 & Sendai & skin fibroblasts & Haupt, 2018 \\
\hline ACT patient 1.2 & ACT 1.10 & Sendai & skin fibroblasts & Haupt, 2018 \\
\hline ACT patient 2.1 & ACT 2.9 & Sendai & skin fibroblasts & Haupt, 2018 \\
\hline ACT patient 2.2 & ACT 2.14 & Sendai & skin fibroblasts & Haupt, 2018 \\
\hline ACT patient 3.1 & ACT 3.1 & Sendai & skin fibroblasts & Haupt, 2018 \\
\hline ACT patient 3.2 & ACT 3.11 & Sendai & skin fibroblasts & Haupt, 2018 \\
\hline Control patient 1.1 & ACT K1.2 & Sendai & skin fibroblasts & Haupt, 2018 \\
\hline Control patient 1.2 & ACT K1.8 & Sendai & skin fibroblasts & Haupt, 2018 \\
\hline Control patient 2.1 & ACT K2.2 & Sendai & skin fibroblasts & Haupt, 2018 \\
\hline Control patient 2.2 & ACT K2.9 & Sendai & skin fibroblasts & Haupt, 2018 \\
\hline DCM 1.6 & sDCM 1.6 & Sendai & skin fibroblasts & Unpublished \\
\hline DCM 1.10 & sDCM 1.10 & Sendai & skin fibroblasts & Unpublished \\
\hline Crt-DCM 2.1 & sDCM 2F1 & Sendai & PBMCs & Unpublished \\
\hline Crt-DCM 2.2 & sDCM 2F2 & Sendai & PBMCs & Unpublished \\
\hline DCM 3.7 & sDCM 3.7 & Sendai & PBMCs & Unpublished \\
\hline CRT1 & pCTRL1.1 & Plasmid & PBMCs & $\begin{array}{l}\text { Borchert et al., } \\
2017\end{array}$ \\
\hline Phoenix & Phoenix & Sendai & $\begin{array}{l}\text { cord blood } \\
\text { cells }\end{array}$ & $\begin{array}{l}\text { Haase et al., } \\
2017\end{array}$ \\
\hline
\end{tabular}

PBMCs: Peripheral blood mononuclear cells

\subsection{Laboratory materials}

3.2.1. Chemicals, kits, and media

Chemicals, commercial kits, and basic cell culture ingredients are listed in Table 3-2 with their respective supplier.

Table 3-2: Chemicals, kits, and media

\begin{tabular}{l|l}
\multicolumn{1}{c|}{ Item } & \multicolumn{1}{c}{ Supplier } \\
\hline 100 bp+ DNA ladder & Thermo Fisher \# 15628019 \\
\hline 10-cm dish, TC-treated & CytoOne Starlab \# CC7682-3394 \\
\hline 12-well plate, TC-treated & CytoOne Starlab \# CC7682-7512 \\
\hline $\begin{array}{l}\text { 4-(2-Hydroxyethyl)piperazine-1- } \\
\text { ethanesulfonic acid (HEPES) }\end{array}$ & Sigma \# H3375 \\
\hline 4.6-diamino-2-phenylindole, 0.2 ng/ml (DAPI) & Sigma-Aldrich \# D9542 \\
\hline 6-cm dish, TC-treated & CytoOne Starlab \# CC7682-3359 \\
\hline 6-well plate, TC- treated & CytoOne Starlab \# CC7682-7506 \\
\hline Acetonitrile, 100\% HPLC-grade & Fisher Scientific \# A996-1 \\
\hline Alt-R Cas9 electroporation enhancer & IDT \# 1075915 \\
\hline Alt-R CRISPR-Cas9 tracrRNA & IDT \# 1072532 \\
\hline Alt-R S.p. Cas9 nuclease V3 & IDT \# 1081058
\end{tabular}


Amaxa nucleofector system, human stem cell Lonza \# VPH-5012 nucleofectorTM kit 1

\begin{tabular}{|c|c|}
\hline $\begin{array}{l}\text { Amersham protran premium } 0.45 \mu \mathrm{m} \\
\text { nitrocellulose blotting membrane }\end{array}$ & $\begin{array}{l}\text { GE Healthcare, Life science } \\
\# 10600003\end{array}$ \\
\hline $\begin{array}{l}\text { Amplex Red Hydrogen Peroxide/Peroxidase } \\
\text { Assay Kit (Amplex red kit) }\end{array}$ & Thermo Fisher Scientific \# A22188 \\
\hline $\begin{array}{l}\text { APC-Annexin V apoptosis detection kit with } \\
\text { PI }\end{array}$ & BioLegend \# 640932 \\
\hline B-27 supplement, $50 x$, serum free & Thermo Fisher Scientific \# 17504044 \\
\hline Black 96-well microplate & Berthold Technologies \# 23302 \\
\hline Boric acid & Sigma-Aldrich \# 15663 \\
\hline $\begin{array}{l}\text { Bovine albumin fraction } V \text { solution (bovine } \\
\text { serum albumin, BSA, } 7.5 \% \text { ) }\end{array}$ & Thermo Fisher Scientific \#15260037 \\
\hline Bradford reagent & Sigma-Aldrich \# B6916 \\
\hline Bromophenol blue & Sigma \# B0126 \\
\hline Cell scraper: 2-Posit. Blade 25 & Sarstedt \# 83.1830 \\
\hline CHIR99021 (CHIR) & Merck Millipore \# 361559 \\
\hline CryoTube vials & Thermo Fisher Scientific \# 377224 \\
\hline Diethylenetriaminepentaacetic acid (DTPA) & Sigma-Adlrich \# D6518 \\
\hline Dimethyl sulfoxide (DMSO) & Sigma-Aldrich \# D2650 \\
\hline Dithiothreitol (DTT) & Sigma \# GE17-1318-01 \\
\hline DMEM-F12 & Thermo Fisher Scientific \# 31331028 \\
\hline Doxorubicin hydrochloride & Sigma-Aldrich \# D1515 \\
\hline Dulbecco's modified Eagle medium (DMEM) & Thermo Fisher Scientific \#11960044 \\
\hline Dulbecco's phosphate-buffered saline (PBS) & Thermo Fisher Scientific \#14190144 \\
\hline $\begin{array}{l}\text { Essential } 8 \text { medium and supplement } \\
\text { (E8 medium) }\end{array}$ & Thermo Fisher Scientific \# A1517001 \\
\hline $\begin{array}{l}\text { Ethylene glycol-bis(2-aminoethylether)- } \\
\mathrm{N}, \mathrm{N}, \mathrm{N}^{\prime}, \mathrm{N}^{\prime} \text {-tetraacetic acid (EGTA) }\end{array}$ & Sigma-Aldrich \# E3889 \\
\hline Ethylenediaminetetraacetic acid (EDTA) & Sigma-Aldrich \# E6758 \\
\hline Falcon round-bottom polystyrene tubes, $5 \mathrm{ml}$ & Stem cell technologies \# 38007 \\
\hline Fetal bovine serum (FBS) & Sigma-Aldrich \# F7524 \\
\hline Filter tips: $0.1-1000 \mu \mathrm{l}$ & $\begin{array}{l}\text { Starlab \#S1120-3810, \# S1122-1830, } \\
\text { \#S1120-1840 }\end{array}$ \\
\hline Fujifilm super RX-N medical X-ray film & Fujifilm \# 4741019289 \\
\hline Gelatin & Sigma-Aldrich \# 48720 \\
\hline Geltrex & Thermo Fisher Scientific \# A1413302 \\
\hline Glass cover slips, round $25 \mathrm{~mm}$ & R. Langenbrinck \# 01-0025/2 \\
\hline Glycine & Sigma \# G8898 \\
\hline Gradient gel, Bolt $4 \%-12 \%$ Bis-Tris Plus & $\begin{array}{l}\text { Thermo Fisher Scientific } \\
\text { \# NW04125BOX }\end{array}$ \\
\hline $\mathrm{H}_{2} \mathrm{O}_{2}[\sim 10 \mathrm{M}]$ & Calbiochem \# 386790 \\
\hline
\end{tabular}


IDTE (1x TE buffer: $10 \mathrm{mM}$ Tris, $0.1 \mathrm{mM}$ EDTA)

Inhibitor of Wnt production 2 (IWP2)

Iscove's modified Dulbecco's medium (IMDM) with GlutaMAX

L-Ascorbic acid 2-phosphate

L-glutamine (200 mM)

Luminol

Methanol

MG-132

Microcentrifuge polypropylene tube

(ultracentrifuge tube)

Midori Green Advance

Monothioglycerol (MTG)

Mr. Frosty freezing container

Non-essential amino acids (NEAA)

Non-fat dry milk

Nuclease-free water

Nucleofector 2b Device

Object slides $(76 \times 26 \mathrm{~mm})$

Oligo d(T)16 (50 $\mu \mathrm{M})$

P-Coumaric acid

Pasteur pipettes, glass

Penicillin/streptomycin

PeqGold universal agarose

Phenylmethanesulfonyl fluoride (PMSF)

Pierce bovine serum albumin standard

ampules, $2 \mathrm{mg} / \mathrm{ml}$, (protein standard)

Polypropylene screw neck cap and preslit

PTFE/silicone septum (HPLC tube)

Ponceau S solution

Protease inhibitor cocktail

Puromycin dihydrochloride (puromycin)

QIAamp DNA mini kit (50)

QIAquick gel extraction kit

QuantiNova reverse transcription kit

Recombinant human basic fibroblast growth

factor (hbFGF)

ReliaPrep RNA tissue miniprep system

Roswell Park Memorial Institute 1640
IDT \# 11-05-01-05

Merck Millipore \# 681671

Thermo Fisher Scientific \# 31980030

Sigma Aldrich \# A8960

Thermo Fisher Scientific \# 25030024

Sigma \# A8511

Merck Millipore \# 106009

Sigma-Aldrich \# 474790

Beckman Coulter \# 357448

Biozym \# 617004

Sigma-Aldrich \# M1753

Thermo Scientific \# 5100-0001

Thermo Fisher Scientific \# 11140035

Roth \# T145

Thermo Fisher Scientific \# AM9932

Lonza \# AAB-1001

Thermo Fisher Scientific

\# 10143562C

Thermo Fisher Scientific \# N808-

0128

Sigma \# C9008

TH Geyer \# 7691061

Thermo Scientific \# 15140-122

Peqlab \# 35-1020

Sigma \# P7626

Thermo Scientific \# 23209

Waters \# 186000305

Sigma-Aldrich \# P7170-1L

Millipore \# 535140

Sigma \# P9620

Qiagen \# 51304

Qiagen \# 28706

Qiagen \# 205413

PeproTech \# 100-18B

Promega \# Z6110

Thermo Fisher Scientific \# 72400047 
Medium (RPMI medium) with HEPES and GlutaMAX

\begin{tabular}{|c|c|}
\hline $\begin{array}{l}\text { Roswell Park Memorial Institute } 1640 \\
\text { Medium (RPMI medium), no glucose }\end{array}$ & Thermo Fisher Scientific \# 11879020 \\
\hline Roti-Histofix 4\% (Histofix) & Carl Roth \# P087.5 \\
\hline Sodium chloride $(\mathrm{NaCl})$ & Sigma \# S7653 \\
\hline Sodium DL-lactate solution $60 \%(\mathrm{w} / \mathrm{w})$ & Sigma-Aldrich \# 2326 \\
\hline Sodium dodecyl sulfate (SDS) & Sigma-Aldrich \# L4509 \\
\hline Sodium fluoride (NaF) & Roth \# P756 \\
\hline Sodium orthovanadate & Sigma-Aldrich \# S6508 \\
\hline StemFlex basal medium (StemFlex medium) & $\begin{array}{l}\text { Thermo Fisher Scientific \# A33493- } \\
01\end{array}$ \\
\hline Steriflip-GP $(0.22 \mu \mathrm{m})$ & Merck Millipore \# SCGP00525 \\
\hline Stripettes: $5 \mathrm{ml}, 10 \mathrm{ml}, 25 \mathrm{ml}, 50 \mathrm{ml}$ & $\begin{array}{l}\text { Sarstedt \# 86.1253.001, } \\
\text { \# 86.1254.001, \# 86.1685.001. } \\
\text { \# 86.1256.001 }\end{array}$ \\
\hline Sucrose & Sigma-Aldrich \# S8501 \\
\hline Superfrost plus adhesion microscope slides & Thermo Scientific \# J1800AMNZ \\
\hline SV total RNA isolation system & Promega \# Z3105 \\
\hline Tetrasodium pyrophosphate $\left(\mathrm{Na}_{4} \mathrm{P}_{2} \mathrm{O}_{7}\right)$ & Merck \# P8010 \\
\hline Thiazovivin (TZV) & Millipore \# 420220 \\
\hline Trifluoroacetic acid (TFA) & Sigma-Aldrich \# 302031 \\
\hline Triton $\mathrm{X}-100$ & Sigma \# X100 \\
\hline Trizma base (Tris) & Sigma \# T1503 \\
\hline Trypsin-EDTA $(0.25 \%)$ & Thermo Fisher Scientific \# 25200056 \\
\hline Tween 20 & Sigma-Aldrich \# P2287 \\
\hline Vectashield mounting medium & $\begin{array}{l}\text { Linaris Biologische Produkte \# H- } \\
1700-2\end{array}$ \\
\hline Versene & Thermo Fisher Scientific \# 15040066 \\
\hline Whatman paper & Sigma-Aldrich \# WHA3030672 \\
\hline White filter cards & Thermo Scientific \# 5991022 \\
\hline$\beta$-Glycerophosphate & Sigma \# 35675 \\
\hline$\beta$-Mercaptoethanol ( $\beta$-ME) & Serva Electrophoresis \# 28625 \\
\hline
\end{tabular}

\section{Millipore water}

Water was filtered with a Milli-Q filter system (Merck, Darmstadt, Germany) to receive ultrapure quality. 
3.2.2. Antibodies for protein detection

Specific antibodies for protein detection using Western blotting were purchased from the suppliers listed in Table 3-3 and diluted as described below.

Table 3-3: List of antibodies for Western blotting

\begin{tabular}{|c|c|}
\hline Antibody & Supplier \\
\hline Anti-GAPDH antibody, monoclonal, mouse, IgM & Sigma-Aldrich \# G8795 \\
\hline Anti-puromycin antibody, monoclonal, mouse, IgG1 kappa & Kerafast, \# EQ0001 \\
\hline Anti- $\beta$-actin antibody, monoclonal, mouse, IgG & Sigma \# A1978 \\
\hline Anti- $\beta$-tubulin antibody, monoclonal, mouse, IgG1 & Sigma-Aldrich \# T8328 \\
\hline Goat anti-mouse-HRP & Millipore \# 401215 \\
\hline $\begin{array}{l}\text { IRDye } 800 \mathrm{CW} \text { donkey anti-mouse IgG secondary } \\
\text { antibody }\end{array}$ & LI-COR \# 926-32212 \\
\hline
\end{tabular}

\section{GAPDH antibody solution}

Anti-GAPDH antibody was diluted 1:10,000 in Tris-buffered saline (TBS)-T, 1\% milk.

\section{Puromycin antibody solution}

Anti-puromycin antibody was diluted 1:1,000 in $5 \mathrm{ml}$ TBS-T.

\section{$\beta$-actin antibody solution}

Anti- $\beta$-actin antibody was diluted $1: 10,000$ in $5 \mathrm{ml} \mathrm{TBS}-\mathrm{T}, 1 \%$ milk.

\section{$\beta$-tubulin antibody solution}

Anti- $\beta$-tubulin antibody was diluted $1: 10,000$ in $5 \mathrm{ml} \mathrm{TBS-T,} 1 \%$ milk.

\section{Goat anti-mouse secondary antibody solution}

Goat anti-mouse antibody was diluted 1:10,000 in $5 \mathrm{ml} \mathrm{TBS-T,} \mathrm{1 \%} \mathrm{milk.}$

Donkey anti-mouse secondary antibody solution

Donkey anti-mouse secondary antibody was diluted 1:15,000 in $5 \mathrm{ml} \mathrm{TBS,} 1 \%$ milk. 
Antibodies for protein detection by immunocytochemistry and their respective dilution ratio are given in Table 3-4.

Table 3-4: List of antibodies for immunofluorescence staining.

\begin{tabular}{l|l|l}
\multicolumn{1}{c|}{ Antibody } & Dilution & \multicolumn{1}{c}{ Supplier } \\
\hline Anti-LIN28, goat, IgG & $1: 300$ & R\&D systems \# AF3757 \\
\hline Anti-NANOG goat, IgG & $1: 200$ & Abcam \# PA5-18406 \\
\hline Anti-OCT4 goat, IgG & $1: 40$ & R\&D systems \# AF1759 \\
\hline Anti-SOX2 mouse, IgG & $1: 50$ & R\&D systems \# MAB2018 \\
\hline Anti-SSEA4 mouse, IgG & $1: 200$ & Abcam \# MC813 \\
\hline Anti-TRA-1-60 mouse, IgM & $1: 200$ & R\&D systems MAB4770 \\
\hline Anti-a-actinin mouse, IgG & $1: 1000$ & Sigma-Aldrich \# A7811 \\
\hline Anti-titin M-Line & $1: 1000$ & Abcam \# ab193218 \\
\hline Goat anti mouse IgM, FITC & $1: 200$ & $\begin{array}{l}\text { Jackson ImmunoResearch } \\
\text { Laboratories \# 115-095-020 }\end{array}$ \\
\hline Donkey anti goat IgG, Alexa Fluor 555 & $1: 1000$ & Thermo Fisher Scientific \# A21432 \\
\hline Donkey anti mouse IgG, Alexa Fluor 488 & $1: 1000$ & Thermo Fisher Scientific \# A21202
\end{tabular}

\section{Antibody solutions for immunofluorescence (IF) staining}

All antibodies for IF staining were diluted in TBS, $1 \%$ milk with the dilution factor specified in Table 3-4.

\subsection{Cell culture media and buffers}

Media, solutions, and buffers were prepared freshly as described below and stored at $+4^{\circ} \mathrm{C}$ for short term or at $-20^{\circ} \mathrm{C}$ for long term.

\section{Amplex red working solution 1}

$1.6 \mu \mathrm{L}$ of $10 \mathrm{mM}$ Amplex red reagent stock solution $+3.2 \mu \mathrm{L} 10 \mathrm{U} / \mathrm{mL}$ HRP stock solution in $315.2 \mu \mathrm{L} 1 \mathrm{x}$ reaction buffer.

\section{Amplex red working solution 2}

$6.5 \mu \mathrm{L} 10 \mathrm{mM}$ Amplex red reagent stock solution $+13 \mu \mathrm{L} 10 \mathrm{U} / \mathrm{mL}$ HRP stock solution in $630.5 \mu \mathrm{L} 1 \mathrm{x}$ reaction buffer.

\section{Blocking buffer}

$5 \%$ milk: $1 \mathrm{~g}$ milk powder in $20 \mathrm{ml}$ TBS-T.

\section{Cardio culture medium}

RPMI medium with HEPES and GlutaMAX (as shipped) and 1x B-27 supplement. 


\section{Cell lysis buffer}

$10 \mathrm{mM}$ HEPES, $50 \mathrm{mM} \mathrm{NaCl}, 0.5 \mathrm{M}$ sucrose, $0.1 \mathrm{mM}$ EDTA, $0.1 \mathrm{mM}$ EGTA, 0.5\%

Triton x 100, 1 mM DTT, $10 \mathrm{mM} \mathrm{Na}_{4} \mathrm{P}_{2} \mathrm{O}_{7}, 100 \mathrm{mM} \mathrm{NaF}, 17.5 \mathrm{mM} \beta$-glycerophosphate, $1 \mathrm{mM}$ PMSF, $10 \mathrm{mM}$ sodium orthovanadate, $5 \mu \mathrm{l} / \mathrm{ml}$ protease inhibitors, and $1 \mu \mathrm{l} / \mathrm{ml} \mathrm{MG}-132 \mathrm{in}$ Millipore water.

\section{Differentiation medium}

RPMI medium with HEPES and GlutaMAX supplemented with $0.02 \%$ L-Ascorbic acid 2phosphate and $0.05 \%$ BSA.

\section{Enhanced chemiluminescence (ECL)}

$100 \mu$ l luminol ( $0.44 \mathrm{~g}$ in $10 \mathrm{ml}$ DMSO) and $44 \mu \mathrm{l}$-coumaric acid ( $0.15 \mathrm{~g}$ in $10 \mathrm{ml}$ DMSO) in $20 \mathrm{ml} 100 \mathrm{mM}$ Tris. Mix well, add $17 \mu \mathrm{H}_{2} \mathrm{O}_{2}$ concentrate (10 mM). Mix well, protect flask from light and store at $4^{\circ} \mathrm{C}$. Re-use up to 5 times within one week.

\section{Freezing medium}

Stem Flex, 10\% DMSO, $2 \mu \mathrm{M}$ TZV.

\section{HbFGF}

$100 \mathrm{mg}$ dissolved in $1 \mathrm{ml}$ of $5 \mathrm{mM}$ Tris and stored at $-20^{\circ} \mathrm{C}$. For use in cell culture the stock was diluted $1: 10$ with $0.1 \%$ BSA for a working concentration of $10 \mathrm{ng} / \mu \mathrm{l}$ and stored at $4{ }^{\circ} \mathrm{C}$.

\section{Iscove medium}

IMDM with GlutaMAX supplemented with $20 \%$ heat-inactivated FBS, 1x NEAA and $450 \mu \mathrm{M}$ MTG.

\section{Running buffer}

$25 \mathrm{mM}$ Tris, $192 \mathrm{mM}$ glycine, 0.1\% SDS in Millipore water

\section{Sample buffer $(4 x)$}

$40 \%$ Glycerol, $40 \%$ SDS, and $1 \mathrm{mM}$ bromophenol blue in $250 \mathrm{mM}$ Tris at $\mathrm{pH} 6.8$ with $100 \mathrm{mM} \mathrm{HCl}$

\section{Selection medium}

RPMI medium without glucose, supplemented with $0.02 \%$ L-Ascorbic acid 2-phosphate, $0.05 \% \mathrm{BSA}$, and $4 \mathrm{mM}$ lactate

\section{TBS}

$10 \mathrm{mM}$ Tris, $150 \mathrm{mM} \mathrm{NaCl}$ in Millipore water, $\mathrm{pH} 7.4$

\section{TBS-T}

TBS + 0.1\% Tween 20 


\section{Transfer buffer}

$25 \mathrm{mM}$ Tris, $192 \mathrm{mM}$ glycine, 10\% methanol in Millipore water

Tris-borat-EDTA (TBE)-buffer

$445 \mathrm{mM}$ Tris base, $445 \mathrm{mM}$ boric acid, 20\% 0.5 EDTA pH 8.0, in Millipore water 


\section{Methods}

\section{1. iPSC culture and passaging}

4.1.1. iPSC culture

iPSCs were cultured with E8 medium with daily media changes on Geltrex-coated dishes in a humidified incubator at $37^{\circ} \mathrm{C}$ and $5 \% \mathrm{CO}_{2}$ (standard conditions). To coat the dishes, $2 \mathrm{mg}$ frozen Geltrex was dissolved in $12 \mathrm{ml}$ ice cold DMEM-F1. The dishes were covered with the Geltrex solution and incubated at $37^{\circ} \mathrm{C}$ for $30-60$ minutes $(\min )$ or at $4^{\circ} \mathrm{C}$ overnight.

\subsection{2. iPSC passaging with Versene on Geltrex}

iPSCs were passaged twice a week to avoid spontaneous differentiation favored by high cell density. Geltrex-coated plates, E8 medium, and Versene were warmed to room temperature (RT). Old media was aspirated, iPSCs were washed twice with Versene solution and carefully incubated with Versene for 3-5 min with minimal movement at RT. When iPSCs began to detach, Versene was aspirated and the cells were detached from the plate with E8 medium supplemented with $2 \mu \mathrm{M} \mathrm{TZV}$. The desired number of iPSCs was transferred onto the Geltrex-coated plates and cultured with E8 and $2 \mu \mathrm{M} \mathrm{ROCK}$ inhibitor TZV under standard conditions over night. The following day the medium was replaced to E8 media without TZV.

iPSCs from DCM 1, ctrl-DCM 2, and gene-corrected ACT-iPSCs were cultured in StemFlex medium. StemFlex medium was changed every other day.

\subsection{Spontaneous differentiation}

Spontaneous differentiation into different germ layers showed the pluripotent capacity of iPSCs. Our media did not contain factors that direct the differentiation into a specific celltype. To generate embryoid bodies (EBs), iPSCs of about $80 \%$ confluency were grown on mouse embryonic fibroblasts (MEFs) feeder layer and then transferred to a non-coated $6 \mathrm{~cm}$ suspension culture dish in DMEM-F12 with GlutaMAX and 20\% Knock-Out Serum Replacement, $1 \%$ NEAA, $1 \% \quad \beta-M E, 10 \mathrm{ng} / \mathrm{ml}$ hbFGF. Cells were incubated at $37^{\circ} \mathrm{C}$ overnight. When EBs had formed, they were carefully washed with Iscove medium, transferred into a non-coated $6 \mathrm{~cm}$ suspension culture dish, and cultivated in Iscove medium in suspension at $37^{\circ} \mathrm{C}$ for eight days. The medium was changed on day four and seven after the start of differentiation. On day eight, about 30 EBs were plated on $0.1 \%$ gelatin-coated culture plates and on $0.1 \%$ gelatin-coated culture plates containing glass cover-slips, respectively. EBs were cultivated for another 25 days in Iscove medium, which was replaced every two days. EBs in the culture plates were used for semiquantitative polymerase chain reaction (PCR).

\subsection{Directed differentiation and cardiomyocyte culture}

For each donor, two iPSC lines were directly differentiated in vitro into $95 \%$ pure cardiomyocytes (CMs) according to protocols in our laboratory (Borchert et al., 2017). 
Differentiation was initiated by culturing iPSCs in differentiation media plus $4 \mu \mathrm{M} \mathrm{CHIR}$ for 48 hours (48 h). CHIR inhibits GSK3 and hence activates the canonical Wnt pathway. After $48 \mathrm{~h}$, the cells were incubated with $5 \mu \mathrm{M}$ IWP2 in differentiation media for another $48 \mathrm{~h}$ to inhibit Wnt signalling. After that, cells were cultured for $48 \mathrm{~h}$ in plain differentiation media and subsequently in cardio culture medium. Cardio culture medium was changed every two days. Three to four weeks after the start of the differentiation the cells were metabolically selected by culturing them in selection media for $4-5$ days. iPSC-CMs were cultured with two media changes per week for at least 60 days to receive matured iPSC-CMs. Five to seven days before the respective experiment, iPSC-CMs were replated to control cell number and to grow them on an appropriate dish. iPSC-CMs were detached from the culture plate with $0.25 \%$ trypsin/EDTA for $5-7 \mathrm{~min}$ at $37^{\circ} \mathrm{C}$. Detached cells were transferred to a $15 \mathrm{ml}$ tube and the trypsin reaction was stopped with FBS. The cell solution was centrifuged for $5 \mathrm{~min}$ at $200 \times \mathrm{g}$. The supernatant was discarded, cells were resuspended in $1 \mathrm{ml} \mathrm{FBS}$, counted in a Thoma-counting chamber, and seeded on Geltrex-coated plates with cardio culture medium, $20 \% \mathrm{FBS}$, and $2 \mu \mathrm{M} \mathrm{TZV}$. After $48 \mathrm{~h}$, the medium was changed to cardio culture. If not indicated otherwise, two- to three-month-old iPSC-CMs were exposed to DOX for $24 \mathrm{~h}$ with concentrations ranging from $0.1 \mu \mathrm{M}$ DOX to $5 \mu \mathrm{M}$ DOX. Further treatment, harvest or other handling was dependent on the assay and is described with the respective experiment.

\subsection{DOX measurements with HPLC}

4.4.1. Sample preparation for intracellular DOX measurement Intracellular DOX levels were analyzed according to an adapted protocol for dihydroethidium (DHE) measurement (Fernandes et al., 2007). DOX-challenged iPSC-CMs were washed twice with PBS plus $100 \mu \mathrm{M}$ DTPA (PBS-DTPA) and immediately lysed with $500 \mu \mathrm{l}$ acetonitrile. Cells were detached from the plate with a cell scraper. Detached lysates were transferred to $1.5 \mathrm{ml}$ tubes and centrifuged at $10,000 \times \mathrm{g}$ for $10 \mathrm{~min}$ in a cooled table-top centrifuge (Eppendorf $5418 \mathrm{R}$ ). The supernatant was transferred to a fresh tube, dried in a vacuum concentrator (Speed Vac with vacuum pump and refrigerated vapor trap, Thermo Fisher Scientific), resuspended in PBS-DTPA, transferred to a $300 \mu \mathrm{HPLC}$ tube, and lined up in a Dionex UltiMate 3000 Autosampler (Thermo Scientific) at $4{ }^{\circ} \mathrm{C}$ in the dark for HPLC measurement (see 4.4.3).

\subsubsection{Subcellular fractionation for DOX detection}

DOX-treated iPSC-CM lysates were fractionated throught ultracentrifugation to isolate particular cellular compartments: 1) mitochondrial and nuclear fraction (mito fraction), 2) membrane fraction, 3) cytoplasmic fraction to determine the subcellular distribution of DOX. The separate fractions were measured individually with HPLC for DOX content. First, two million DOX-treated iPSC-CMs (1 $\mu \mathrm{M}$ DOX for $24 \mathrm{~h}$ ) were harvested, lysed in $150 \mu \mathrm{l}$ Tris- $\mathrm{HCl}$ $0.1 \mathrm{M}$ lysis buffer, and sonicated $2 \times 10 \mathrm{~s}$ at 8 Watts with a Branson Sonifier 150 . Subcellular 
fractionation was achieved with ultracentrifugation as described before (Laurindo et al., 2002) with minor changes. To receive the mito fraction, sonicated lysates were centrifuged at $18,000 \times \mathrm{g}$ for $30 \mathrm{~min}$ in a table-top centrifuge (Eppendorf, $5418 \mathrm{R}$ ) at $4^{\circ} \mathrm{C}$. $140 \mu \mathrm{l}$ of the supernatant of each condition were transferred to a $1.5 \mathrm{ml}$ ultracentrifuge tube, and centrifuged at 47,000 revolutions per minute (rpm) for $1 \mathrm{~h}$ at $4^{\circ} \mathrm{C}$ in a Beckman Coulter Optima MAX centrifuge with a TLA-55 rotor. The resulting pellet was the membrane fraction. The supernatant was transferred to a new $1.5 \mathrm{ml}$ eppendorf tube and marked as 'cytoplasmic fraction' (Figure 4-4).

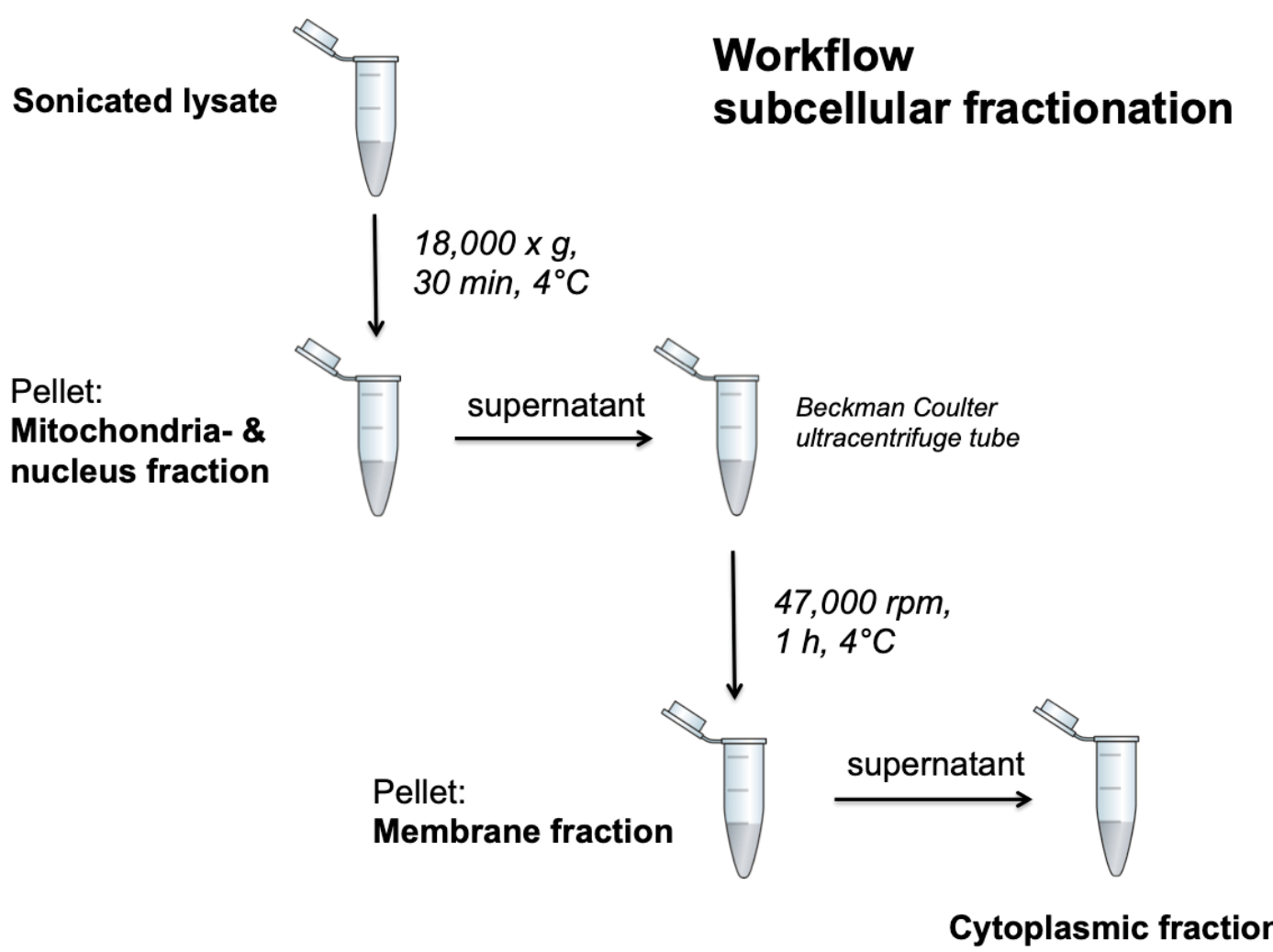

Figure 4-4: Schematic overview of the membrane isolation workflow.

The mito fraction pellets and the membrane fraction pellets were resuspended in $100 \mu \mathrm{l}$ PBS and sonicated. Five $\mu \mathrm{l}$ from each fraction were used for a Bradford assay to determine protein concentration as reference (see 4.6.1). $100 \mu \mathrm{l}$ acetonitrile were added to the rest of the samples, and the tubes were immediately vortexed for $10 \mathrm{~s}$. Then, the samples were centrifuged at $18,000 \times \mathrm{g}$ for $15 \mathrm{~min}$ in a table-top centrifuge (Eppendorf) at $4^{\circ} \mathrm{C}$. The supernatant was transferred to a new tube and dried in a Speed Vac with vacuum pump and a refrigerated vapor trap (Thermo Fisher Scientific). Dried samples were resuspended in 100 $\mu \mathrm{I}$ PBS and measured with HPLC.

4.4.3. HPLC measurement and data processing The HPLC set-up consisted of the following units: Flow manager, UV unit, pump (all Dionex), and a Micro LC column (Phenomenex, Torrance, CA, USA). Peak capacity was increased 
with a Thermo Scientific nanoViper connection. Injection volume was $25 \mu$ per sample and the flow rate was set to $0.400 \mathrm{ml} / \mathrm{min}$ with a pressure of approximately 70 bar. Solution $A$ was $>99 \%$ HPLC-grade acetonitrile with $0.1 \%$ TFA and solution B was Millipore grade l-filtered water with $0.1 \%$ TFA. Initial solution $A$ to solution $B$ ratio was $85 \%: 15 \%$, gradually shifting to $15 \%: 85 \%$ over the measurement period of $40 \mathrm{~min}$. DOX peaks were identified by a reference injection of diluted DOX and appeared after a retention time of approximately $16 \mathrm{~min}$. For each peak the area under the curve was considered. Peak analysis was carried out with Chromeleon 7 software.

\subsection{Nucleic acid isolation and PCR}

\subsubsection{RNA isolation}

Pellets of iPSCs and embryonic bodies, respectively, were snap frozen and stored at $-80^{\circ} \mathrm{C}$. The RNA was isolated with the SV Total RNA Isolation System according to the manufacturer's protocol. Briefly, the pellets were lysed with 400-600 $\mu$ RNA lysis buffer supplemented with $\beta-\mathrm{ME}(20 \mu \mathrm{l} / \mathrm{ml})$. Equal volumes of $95 \%$ ethanol were added to the lysate and the sample was transferred to a spin-basket tube. The samples were washed with RNA wash solution and incubated with DNase I mix for 15 min at RT. The reaction was stopped with $200 \mu$ of DNase stop solution. The samples were washed twice and the RNA was eluted with $100 \mu$ nuclease-free water. RNA concentration was determined with a NanoPhotometer N60 (IMPLEN, Munich, Germany) at 260/280 nm and the samples were stored at $-80^{\circ} \mathrm{C}$.

\subsubsection{DNA isolation with QIAamp DNA Mini Kit} Genomic DNA was isolated from iPSCs with the QIAamp DNA Mini Kit according to the manufacturer's instructions. The genomic DNA was eluted in $100 \mu \mathrm{l}$ nuclease-free water. The genomic DNA yield was measured with a NanoPhotometer N60 (IMPLEN, Munich, Germany) at $260 / 280 \mathrm{~nm}$ and the samples were stored at $-80^{\circ} \mathrm{C}$.

\subsubsection{DNA amplification with PCR}

To synthesize complementary DNA (cDNA) from our isolated RNA, we used the QuantiNova Reverse Transcription Kit according to the manufacturer's protocol. In a first step to remove residual genomic DNA from the isolated RNA, $100 \mathrm{ng}$ RNA and $1 \mu \mathrm{l}$ of genomic DNA removal Mix $(1 \mathrm{x})$ were mixed in nuclease-free water to a total volume of $15 \mu \mathrm{l}$. The mix was incubated at $45^{\circ} \mathrm{C}$ for $2 \mathrm{~min}$ and placed on ice immediately. For reverse transcription, genomic DNA-free RNA (15 $\mu \mathrm{l}), 2 \mu \mathrm{l}$ of the reverse transcription mix (1x), and $0.5 \mu \mathrm{l}$ of the reverse transcription enzyme were mixed in nuclease-free water to a total volume of $20 \mu$. The reverse transcription mix was incubated in a PCR cycler at $25^{\circ} \mathrm{C}$ for $3 \mathrm{~min}$, at $45^{\circ} \mathrm{C}$ for $10 \mathrm{~min}$ and eventually at $85^{\circ} \mathrm{C}$ for $5 \mathrm{~min}$. For extended storage, synthesized cDNA was placed at $-20^{\circ} \mathrm{C}$. 
Amplification of genomic DNA and cDNA sequences was done with PCR. The PCR, first described in 1986 (Mullis et al., 1986), contained the following three basic reaction steps, which are repeated in various cycles: 1) denaturation of introduced DNA, 2) annealing of oligonucleotides at the matrix, and 3) amplification of the target DNA sequence. To initiate amplification, two single stranded oligonucleotides (primers) were needed that served as a start signal for the DNA-polymerase. One of these oligonucleotides is complementary to the sequence at the 5' end of the sequence to be amplified (forward primer; for), one is complementary to the 3 ' end of the opposite strand (reverse primer; rev). Components of the PCR reaction mix (Table 4-1) and details of the reaction steps (Table 4-2) are displayed below.

Table 4-1: Required components and their volume for $P C R$

Table 4-2: Reaction steps during PCR

\begin{tabular}{l|l} 
Components for PCR & $\begin{array}{l}\text { Volume for } \\
\text { one reaction } \\
(\mathbf{2 5} \boldsymbol{\mu l} \text { final) }\end{array}$ \\
\hline cDNA & $1 \mu \mathrm{l}$ \\
\hline Nuclease-free $\mathrm{H}_{2} \mathrm{O}$ & $15.3 \mu \mathrm{l}$ \\
\hline $\begin{array}{l}5 x \text { Green GoTaq } \\
\text { reaction buffer }\end{array}$ & $5 \mu \mathrm{l}$ \\
\hline 10 mM dNTPs & $1.6 \mu \mathrm{l}$ \\
\hline Forward primer $(10 \mu \mathrm{M})$ & $1 \mu \mathrm{l}$ \\
\hline Reverse primer $(10 \mu \mathrm{M})$ & $1 \mu \mathrm{l}$ \\
\hline $\begin{array}{l}\text { GoTag DNA } \\
\text { polymerase }\end{array}$ & $0.1 \mu \mathrm{l}$
\end{tabular}

\begin{tabular}{l|l|l} 
Process & Temperature & Time \\
\hline Denaturing & $95^{\circ} \mathrm{C}$ & $3 \mathrm{~min}$ \\
\cline { 2 - 3 } & $94^{\circ} \mathrm{C}$ & $15 \mathrm{~s}$ \\
\hline Annealing & $\begin{array}{l}\text { Listed in } \\
\text { Table 4-3 }\end{array}$ & $15 \mathrm{~s}$ \\
\hline Synthesis & $72^{\circ} \mathrm{C}$ & $30 \mathrm{~s}$ \\
\cline { 2 - 3 } & $72^{\circ} \mathrm{C}$ & $10 \mathrm{~min}$ \\
\hline End & $4^{\circ} \mathrm{C}$ & $\infty$
\end{tabular}

Detailed primer sequences and reaction mixes for reverse transcription and PCR are listed in Table 4-3.

Table 4-3: Primer sequences for PCR primers.

\begin{tabular}{|c|c|c|c|c|c|c|}
\hline Target & & Sequence $\left(5^{\prime} \rightarrow 3^{\prime}\right)$ & $\begin{array}{l}\text { Product size } \\
\text { (base pairs) }\end{array}$ & $\begin{array}{l}\text { Annealing } \\
\text { temperatur } \\
\text { e }\left[{ }^{\circ} \mathrm{C}\right]\end{array}$ & $\begin{array}{l}\text { Cycle } \\
\text { s }\end{array}$ & Used for \\
\hline \multirow[t]{2}{*}{ AFP } & For: & $\begin{array}{l}\text { ACTCCAGTAAACC } \\
\text { CTGGTGTTG }\end{array}$ & 255 & 60 & 33 & PCR \\
\hline & Rev: & $\begin{array}{l}\text { GAAATCTGCAATG } \\
\text { ACAGCCTCA }\end{array}$ & & & & \\
\hline \multirow[t]{2}{*}{$A L B$} & For: & $\begin{array}{l}\text { CCTTTGGCACAAT } \\
\text { GAAGTGGGTAACC }\end{array}$ & 355 & 62 & 43 & $\begin{array}{l}\text { PCR } \\
\text { PCR }\end{array}$ \\
\hline & Rev: & $\begin{array}{l}\text { CAGCAGTCAGCCA } \\
\text { TTTCACCATAGG }\end{array}$ & & & & \\
\hline cTNT & For: & $\begin{array}{l}\text { GACAGAGCGGAAA } \\
\text { AGTGGGA }\end{array}$ & 305 & 62 & 26 & PCR \\
\hline
\end{tabular}




\begin{tabular}{|c|c|c|c|c|c|c|}
\hline & Rev: & $\begin{array}{l}\text { TGAAGGAGGCCA } \\
\text { GGCTCTAT }\end{array}$ & & & & \\
\hline \multirow[t]{2}{*}{ GAPDH } & For: & $\begin{array}{l}\text { AGAGGCAGGGAT } \\
\text { GATGTTCT }\end{array}$ & 258 & 60 & 30 & PCR \\
\hline & Rev: & $\begin{array}{l}\text { TCTGCTGATGCCC } \\
\text { CCATGTT }\end{array}$ & & & & \\
\hline \multirow[t]{2}{*}{ HPRT } & For: & $\begin{array}{l}\text { CAAAGATGGTCAA } \\
\text { GGTCGC }\end{array}$ & 81 & 60 & 40 & PCR \\
\hline & Rev: & $\begin{array}{l}\text { CAAATCCAACAAA } \\
\text { GTCTGGCT }\end{array}$ & & & & \\
\hline \multirow[t]{2}{*}{ LIN28 } & For: & $\begin{array}{l}\text { AGTAAGCTGCACA } \\
\text { TGGAAGG }\end{array}$ & 410 & 52 & 30 & PCR \\
\hline & Rev: & $\begin{array}{l}\text { ATTGTGGCTCAAT } \\
\text { TCTGTGC }\end{array}$ & & & & \\
\hline \multirow[t]{2}{*}{ NANOG } & For: & $\begin{array}{l}\text { AGTCCCAAAGGCA } \\
\text { AACAACCCACTTC }\end{array}$ & 164 & 64 & 36 & PCR \\
\hline & Rev: & $\begin{array}{l}\text { ATCTGCTGGAGGC } \\
\text { TGAGGTATTTCTG } \\
\text { TCTC }\end{array}$ & & & & \\
\hline \multirow[t]{2}{*}{ OCT4 } & For: & $\begin{array}{l}\text { GACAACAATGAAA } \\
\text { ATCTTCAGGAGA }\end{array}$ & 473 & 59 & 36 & PCR \\
\hline & Rev: & $\begin{array}{l}\text { TTCTGGCGCTTAC } \\
\text { AGAACCA }\end{array}$ & & & & \\
\hline \multirow[t]{2}{*}{ RAC2 } & For: & $\begin{array}{l}\text { TTGCCCTGAGAAC } \\
\text { CAAGACC }\end{array}$ & 299 & 60 & 33 & $\begin{array}{l}\text { PCR, } \\
\text { sequencing }\end{array}$ \\
\hline & Rev: & $\begin{array}{l}\text { CGCTGCTATTTCA } \\
\text { TGGCTGG }\end{array}$ & & & & \\
\hline \multirow[t]{2}{*}{ SOX2 } & For: & $\begin{array}{l}\text { ATGCACCGCTACG } \\
\text { ACGTGA }\end{array}$ & 437 & 60 & 34 & PCR \\
\hline & Rev: & $\begin{array}{l}\text { CTTTTGCACCCCT } \\
\text { CCCATTT }\end{array}$ & & & & \\
\hline \multirow[t]{2}{*}{$T H$} & For: & $\begin{array}{l}\text { GCGGTTCATTGGG } \\
\text { CGCAGG }\end{array}$ & 215 & 60 & 34 & PCR \\
\hline & Rev: & $\begin{array}{l}\text { CAAACACCTTCAC } \\
\text { AGCTCG }\end{array}$ & & & & \\
\hline
\end{tabular}

4.5.4. Agarose gel electrophoresis

To determine the presence and size of amplified DNA sequences, PCR products were fractioned with gel electrophoresis. The samples and a DNA ladder were pipetted into the pockets of an $1.5 \%$ TBE-agarose gel with $0.004 \%$ Midori green. The gel was run in TBE buffer at $120 \mathrm{~V}$ for $20 \mathrm{~min}$. Fluorescence was captured with a Chemidoc XRS+ imager (BioRad, Feldkirchen, Germany).

\section{6. $\quad$ mRNA translation assay with puromycin and Western blotting}

4.6.1. Cell lysis and sample preparation

iPSC-CMs were treated with $1 \mu \mathrm{M}$ DOX in $1.5 \mathrm{ml} /$ well cardio culture medium for $24 \mathrm{~h}$. Untreated controls were cultured in $1.5 \mathrm{ml} /$ well cardio culture medium without DOX.

Before harvesting, $1 \mu \mathrm{M}$ puromycin $(7.5 \mu \mathrm{l}$ from $200 \mu \mathrm{M}$ stock in $1.5 \mathrm{ml}$ medium) was added to each well and iPSC-CMs were incubated for $20 \mathrm{~min}$ at $37^{\circ} \mathrm{C}$ in the dark. iPSC-CMs were rinsed 
twice with PBS, and harvested with $120 \mu$ l cell lysis buffer. Lysates were sonicated and protein content was determined with Bradford reagent according to the manufacturer's recommendation. Briefly, $5 \mu \mathrm{l}$ of protein samples or a protein standard dilution series were pipetted in triplicates onto a 96-well plate. $250 \mu$ of the Bradford reagent were added per well and the samples were incubated for $10 \mathrm{~min}$ at RT. Afterwards, the absorption was detected photospectroscopically with a plate reader (Tecan, Männedorf, Switzerland) at $595 \mathrm{~nm}$. The absorption values of the protein standard series were used to calculate a standard curve. The standard curve was used to calculate sample protein concentrations from the absorption values of the samples.

During the incubation time of the Bradford assay, $40 \mu \mathrm{l}$ of $4 \mathrm{x}$ sample buffer and $5 \% \beta$-ME (8 $\mu \mathrm{l}$ per sample) were added to the iPSC-CMs protein samples. They were denaturated for $5 \mathrm{~min}$ at $90^{\circ} \mathrm{C}$ and placed on ice to cool down.

4.6.2. SDS-gel electrophoresis and Western blot

Proteins were separated with SDS-polyacrylamide electrophoresis as described before (Laemmli, 1970). To this end, equal amounts of protein (15 $\mu \mathrm{g}, 8-15 \mu \mathrm{l}$ of lysate) were loaded into the pockets of a precast gradient gel (4-12\%). The gel was run for approximately $45 \mathrm{~min}$ at $160 \mathrm{~V}$ (PowerPac Basic Power Supply, BioRad) in 1x running buffer. After the run, the gel was taken out of the casing and the proteins were transferred to a nitrocellulose membrane with the Western blot approach (Towbin et al., 1979). The transfer block contained (from bottom to top) sponge, Whatman paper, the polyacrylamide gel, nitrocellulose blotting membrane, Whatman paper, and sponge. All parts were briefly washed in transfer buffer before assembly. The transfer assembly was placed into a transfer chamber (Bio-Rad) together with an ice-pad and transfer buffer. The transfer ran for $80 \mathrm{~min}$ at $70 \mathrm{~V}$ (PowerPac Basic Power Supply, Bio-Rad). After blotting, the membrane was taken out, cut to size and stained with Ponceau Red solution to confirm successful transfer.

\subsubsection{Protein detection with specific antibodies}

Ponceau staining was washed out with water and the membrane was blocked in $20 \mathrm{ml}$ blocking buffer for $1 \mathrm{~h}$. The membrane was incubated with primary anti-puromycin antibody solution over-night on a shaker at $4{ }^{\circ} \mathrm{C}$. The following day, the membrane was washed 3 times for 15 min with TBS-T and incubated with the secondary antibody for $45 \min$ to $1 \mathrm{~h}$ at RT. Afterwards, the membrane was washed three times for 15 min with TBS-T. After the last washing step, the membrane was incubated with $2-3 \mathrm{ml}$ of ECL solution for $2 \mathrm{~min}$. Then, the ECL solution was removed, the membrane was dabbed and luminescence was captured with a medical X-ray film. Exposure times varied according to the antibody. Subsequently, the film was developed in an automated developer (X-Ray film Processor, Optimax 2010; Protec Oberstenfeld, Germany). 
For loading control, the membrane was rehydrated in Millipore water, blocked in blocking solution for $1 \mathrm{~h}$ at RT and incubated overnight at $4^{\circ} \mathrm{C}$ with antibodies directed against glyceraldehyde 3-phosphate dehydrogenase (GAPDH), $\beta$-actin, or $\beta$-tubulin. The following day, the membrane was washed three times with PBS for $15 \mathrm{~min}$, incubated with LICOR IRDye $800 \mathrm{CW}$ donkey anti-mouse IgG secondary antibody (1:15,000) in TBS for 40 min at RT, and then washed three times with TBS. Fluorescent signals were measured with an Odyssey CLx Imaging System (LI-COR).

Signal quantification was performed with FIJl. Average lane intensities were normalized to reference band intensities.

\subsection{Genome editing}

\subsubsection{Sequence design and iPSCs preparations}

To edit the SNP in the RAC2 gene, we chose the CRISPR/Cas9 system provided by IDT. Guide RNA and homology-directed repair (HDR)-template were designed with IDT and mismatch scores were tested with CRISPOR. The final sequence for the guide RNA was selected based on the distance of the cutting site to the SNP and the predicted mismatches. We chose the guide RNA which had the closest cutting site to the SNP (5 base pairs) and a low number of predicted mismatches. Based on the guide RNA, the HDR template was designed and both were ordered from IDT.

iPSCs from our target cell line ACT1.10 were thawed and cultured for at least one week before genome editing. The passage of the used iPSC line did not exceed 20 . On the day of the transfection, the iPSCs had a confluency of 70-80\% (1-2 million iPSCs per 6-well). Before transfection, cell morphology and density were documented by taking brightfield images with an Axio Oberserver light microscope (Carl Zeiss, 4x and 10x magnification).

\subsubsection{Complex formation and transfection preparation}

In preparation of the assay, guide RNA, Alt-R CRISPR-Cas9 tracrRNA, and the Alt-R Cas9 Electroporation enhancer were resuspended in TE buffer to an $100 \mu \mathrm{M}$ stock solution, respectively. Five $\mu \mathrm{l}$ of the guide RNA stock $(100 \mu \mathrm{M})$ and $5 \mu \mathrm{l}$ of the Alt-R CRISPR-Cas9 tracrRNA stock $(100 \mu \mathrm{M})$ were mixed in a $0.5 \mathrm{ml}$ reaction tube and incubated at $95^{\circ} \mathrm{C}$ for $5 \mathrm{~min}$. The mixture was cooled down to RT. For final complex formation, $6 \mu$ l of the CRISPRRNA mix and $2 \mu$ of the Cas 9 Nuclease were mixed in a $1.5 \mathrm{ml}$ tube and incubated for $20 \mathrm{~min}$ at RT. Then, the complex was warmed to $37^{\circ} \mathrm{C}$ in the incubator. The CRISPR-RNACas9 complex, the Alt-R Cas9 Electroporation enhancer, the nucleofector solution and the HDR template (resuspended in IDTE to $100 \mu \mathrm{M}$ stock) were mixed in a $1.5 \mathrm{ml}$ reaction tube according to Table 4-4. 
Table 4-4: Components of the nucleofector solution.

\begin{tabular}{l|l} 
Component & Amount \\
\hline Nucleofector solution & $82 \mu \mathrm{l}$ \\
\hline Nucleofector supplement & $18 \mu \mathrm{l}$ \\
\hline CRISPR-RNA-Cas9 complex & $8 \mu \mathrm{l}$ \\
\hline $\begin{array}{l}\text { Alt-R Cas9 electroporation enhancer } \\
(100 \mu \mathrm{M})\end{array}$ & $1 \mu \mathrm{l}$ \\
\hline HDR template $(100 \mu \mathrm{M})$ & $3 \mu \mathrm{l}$
\end{tabular}

After appropriate mixing, the solution was incubated for 5 min at RT. Before use, it was warmed to $37^{\circ} \mathrm{C}$.

\subsubsection{Transfection}

iPSCs from the ACT1.10 line were pretreated with $2 \mu \mathrm{M}$ TZV for $60 \mathrm{~min}$ before transfection. iPSCs were detached from the plate with Versene and washed off the plate with StemFlex medium. Two million iPSCs were transferred into a new $15 \mathrm{ml}$ falcon tube and spun down for three min at $200 \times \mathrm{g}$. The supernatant was aspirated and the cells were resuspended in the prepared nucleofector mix. The mixture was then transferred to a nucleofector cuvette and transfected with program B-016 of the Nucleofector 2b Device. The iPSCs were then diluted in $1 \mathrm{ml}$ StemFlex medium and plated equally on three new wells of a 6-well plate with Stem Flex/TZV and Penicillin/Streptomycin. To test the efficacy of an HDR template enhancer, we added $20 \mu \mathrm{M}$ and $30 \mu \mathrm{M}$ HDR template enhancer to two wells of the 6-well plate, respectively. One well was not treated with HDR template enhancer and served as negative control. The transfected cells were cultured for $48 \mathrm{~h}$ at $37^{\circ} \mathrm{C}$ and $5 \% \mathrm{CO}_{2}$. Then, the medium was changed to StemFlex medium with $2 \mu \mathrm{M}$ TZV. On day six after transfection, iPSCs were singularized and the rest was harvested for amplicon sequencing and cryo-preserved (4.7.4).

\subsubsection{Singularization of the transfected cells}

In order to obtain single cell colonies, transfected iPSCs were singularized through Versene treatment and pipetted up and down for two to four times. iPSCs were counted and 600, 800, $1000,1200,1400$, and 1600 cells were seeded onto a well of 6 -well plate, respectively. One half of the remaining cells was centrifuged for three $\min$ at $200 \times \mathrm{g}$, the supernatant aspirated, resuspended in freezing medium and cryo-preserved with a Mr. Frosty Freezing Container at $-80^{\circ} \mathrm{C}$. The other half of the remaining cells was centrifuged at $13,000 \mathrm{rpm}$ for one min, the supernatant was aspirated and the pellet snap-frozen in liquid nitrogen. 


\subsubsection{Picking colonies}

After approximately one week, single iPSC colonies were picked with heat-modified glass Pasteur pipettes and each colony was transferred to one well of a 12-well plate, respectively. Singularized colonies were cultured with Stem Flex media until they were confluent. Confluent colonies were transferred to a well of a 6-well plate and once that was confluent, iPSCs were harvested for Sanger sequencing and for cryo-preservation.

\subsubsection{Sanger sequencing}

To test the genomic status of gene-edited iPSC colonies, iPSCs were harvested and sent for Sanger sequencing (Microsynth seqlab). iPSCs were harvested from one well of a 6-well plate when they were confluent and genomic DNA (gDNA) was isolated as described under 4.5.2. The region around the SNP was amplified with Rac2-specific primers with PCR (4.5.3), and PCR products were reviewed for matching band size and purity with gel electrophoresis (4.5.4). Rac2 bands were cut out of the agarose gel and the PCR product was purified with a QIAquick gel extraction kit according to the manufacturer's instructions. The purified product was sent to Microsynth seqlab for Sanger sequencing and results were processed with the BioEdit software.

\subsection{Telomere length and TERT overexpression}

\subsubsection{Telomere length assay with $P C R$}

Telomere length was compared between different iPSC lines and between iPSCs and iPSCCMs of the same line with PCR as reported by Cawthon (Cawthon, 2002). In this approach, the Telomere-to-Single Copy Gene (T/S) ratio is determined, which is proportional to the average telomere length in a cell (Cawthon, 2002). The described protocol was optimized following reported improvements (Martinez-Delgado et al., 2011). Telomere length assays were carried out by Shambhabi Chatterjee in the group of Dr. Chrisitan Bär at the Medizinische Hochschule Hannover. The exact procedure was recently described in detail by our collaborators (Chatterjee et al., 2018). In short, DNA from iPSC and iPSC-CMs was extracted and amplified with primers for the telomere repeat sequence and a reference single copy gene (36B4) by PCR. The relative telomere length was determined by the ratio of the telomere repeat sequence to the reference single copy gene.

4.8.2. Telomere length assay with quantitative fluorescent in situ hybridization Our group prepared the cells for the quantitative fluorescent in situ hybridization (qFISH) analysis: iPSCs and iPSC-CMs were detached with Versene solution and trypsin/EDTA, respectively. For each cell type two concentrations were prepared. For iPSCs one solution contained $7.5 \times 10^{5}$ cells $/ \mathrm{ml}$ and the other $5 \times 10^{5} \mathrm{cell} / \mathrm{ml}$. For iPSC-CMs the concentrations were $1 \times 10^{6} \mathrm{cells} / \mathrm{ml}$ and $7.5 \times 10^{5} \mathrm{cells} / \mathrm{ml}$. $100 \mu \mathrm{l}$ of each solution were spun on Superfrost microscope slides with white filter cards in a Cytospin 4 centrifuge (Thermo Scientific) at 800 rpm for $5 \mathrm{~min}$. Samples were air-dried and stored at $4^{\circ} \mathrm{C}$. qFISH assays were carried out by 
Shambhabi Chatterjee in the group of Dr. Chrisitan Bär at the Medizinische Hochschule Hannover according to previously reported protocols (Canela et al., 2007). In short, cells were incubated with a hybridization solution containing a fluorophore-conjugated peptide nucleic acid probe. After hybridization, samples were imaged with a fluorescent microscope. Quantitative image analysis of fluorescence intensities was applied to calculate the relative telomere length.

\subsubsection{AAV6 treatment}

TERT overexpression in the iPSC-CMs was induced with AAV6 vectors. AAV6 vectors were produced in the group of Dr. Christian Bär at the Medizinische Hochschule Hannover. AAV6 human TERT (hTERT) contains cytomegalovirus (CMV) promoter and enhancer sequences. Furthermore, it contains ampicillin resistance and the sequence is flanked by AAV2 inverted terminal repeats (ITRs; Figure 4-1). Similarly, AAV6 empty vector is also regulated through a CMV promoter and enhancer. It has ampicillin resistance, and AAV2 ITRs (Figure 4-2). Instead of the hTERT sequence, the empty vector has a poly(A) signal (Figure 4-2). To correctly calculate the multiplicity of infection (MOI), iPSC-CMs were counted and $5 \times 10^{5}$ cells/well were seeded onto a 6-well-plate. Three days later, the medium was changed to cardio culture medium ( $2 \mathrm{ml} /$ well) and another $24 \mathrm{~h}$ later AAV6 at an MOI of $1 \times 10^{4}$ units/cell was added diluted in fresh cardio culture medium $(2 \mathrm{ml} /$ well).

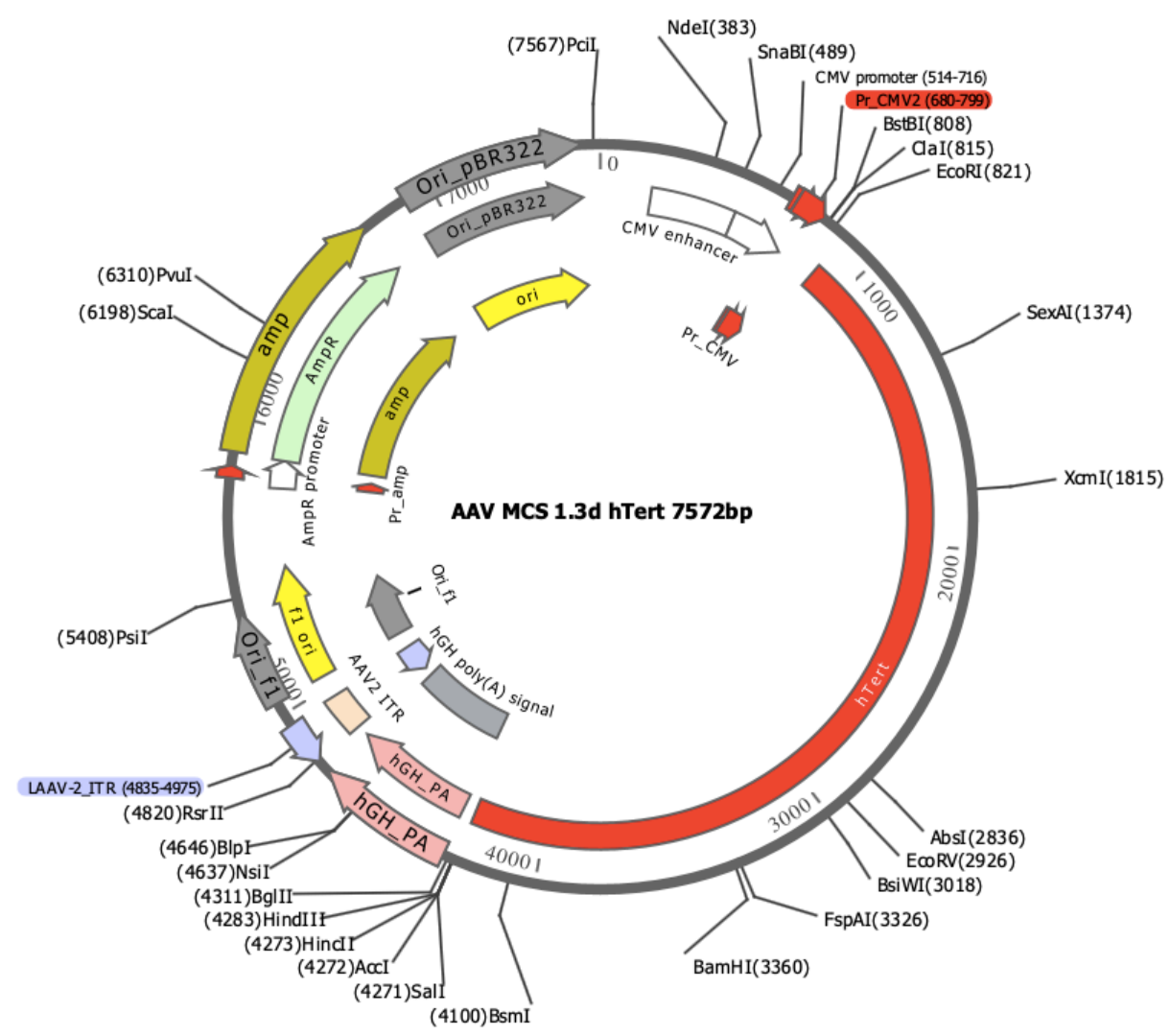

Figure 4-1: Circular map of the AAV6 hTERT vector. 
The AAV6 hTERT vector contained a CMV enhancer and promoter, the hTERT sequence, ampicillin resistance, and AAV2 inverted terminal repeat sequences.

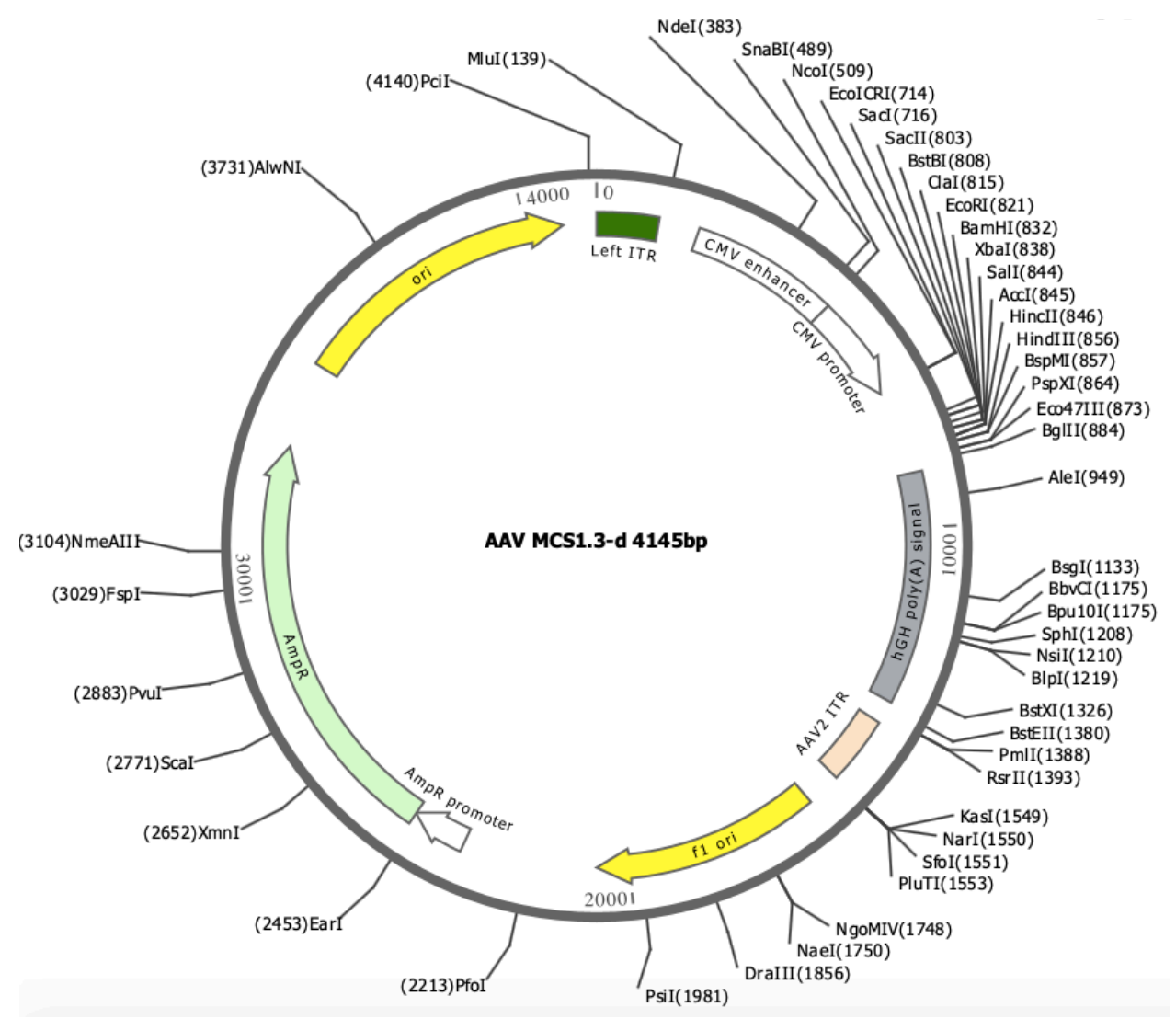

Figure 4-2: Circular map of AAV6 empty vector.

The AAV6 empty vector contained a CMV enhancer and promoter, a poly(A) signal, ampicillin resistance, and AAV2 inverted terminal repeat sequences.

After three days, the medium was changed to virus-free cardio culture medium and iPSCCMs were cultured for seven days to recover from virus treatment. After the recovery period, cells were treated with $0.5 \mu \mathrm{M}$ DOX for three days and subsequently the apoptosis and the Amplex red assays were carried out respectively.

\subsubsection{Apoptosis assay with flow cytometry}

The rate of apoptosis in our samples was observed with the APC-Annexin $V$ apoptosis detection kit. The cells were prepared according to the manufacturer's protocol. Briefly, for each, condition we used $1.5 \times 10^{5}$ iPSC-CMs. iPSC-CMs were washed with PBS and incubated with $0.25 \%$ Trypsin/EDTA for $12 \mathrm{~min}$ to disassociate. To get a complete picture of apoptosis and necrosis, the supernatant was transferred into a conical tube prepared with $500 \mu \mathrm{FCS}$. The cells were segregated from the supernatant through centrifugation at $200 \mathrm{xg}$ for $3 \mathrm{~min}$. The samples were resuspended in $1 \mathrm{ml} \mathrm{PBS}$ and subsequently washed three times. After the last washing step, iPSC-CMs were resuspended in Annexin $\mathrm{V}$ binding buffer. $100 \mu \mathrm{l}$ of the suspension were transferred into one of four flow cytometry tubes, respectively. APC-Annexin $\mathrm{V}(5 \mu \mathrm{l})$ and propidium iodide $(\mathrm{Pl} ; 10 \mu \mathrm{l})$ were added to the tubes 
according to the following scheme: control (none), Annexin single staining (Annexin V), PI single staining $(\mathrm{PI})$, and double staining (Annexin $+\mathrm{PI})$. The staining-iPSC-CM solutions were incubated for $15 \mathrm{~min}$ in the dark at RT. Afterwards, $400 \mu \mathrm{l}$ of Annexin $\mathrm{V}$ binding buffer were added to each tube and the samples were measured with a BD FACS Canto II (BD Biosciences) flow cytometer. Unstained and single stainings served as references for negative and Annexin-/PI-positive cell populations, respectively. Flow cytometry data was analyzed with Flowing Software 2.0.

\subsubsection{Extracellular ROS measurement}

Extracellular $\mathrm{H}_{2} \mathrm{O}_{2}$ in the supernatant of iPSC-CMs was assessed with an Amplex red kit (Zhou et al., 1997). In preparation for the assay, $\mathrm{H}_{2} \mathrm{O}_{2}$ in increasing concentrations from $0 \mu \mathrm{M}$ to $1 \mu \mathrm{M}\left(10 \mu \mathrm{M} \mathrm{H}_{2} \mathrm{O}_{2}\right.$ stock solution diluted in Amplex red reaction buffer) was pipetted into the first row of a black 96-well plate, as depicted in Table 4-5. The values of these wells were used to calculate a standard curve. $\mathrm{H}_{2} \mathrm{O}_{2}$ values were calculated according to the standard curve measurements. In total, $5 \times 10^{5}$ iPSC-CMs per well were cultured in a 6 -well plate and treated with DOX and AAV6 as indicated. On the day of the assay, iPSC-CMs were washed twice with reaction buffer and incubated with $320 \mu \mathrm{l}$ working solution 1 for $30 \mathrm{~min}$ in the dark at $37^{\circ} \mathrm{C}$. Immediately after treatment of the cells, $50 \mu$ of working solution 2 were added to each well of the $\mathrm{H}_{2} \mathrm{O}_{2}$ standards, and the plate was incubated together with the iPSC-CMs for $30 \mathrm{~min}$ in the dark at $37^{\circ} \mathrm{C}$.

Table 4-5: $\mathrm{H}_{2} \mathrm{O}_{2}$ standard for Amplex red measurement

\begin{tabular}{l|l|l|l|l|l|l|l|l|l|l|l}
$\mathbf{1}$ & $\mathbf{2}$ & $\mathbf{3}$ & $\mathbf{4}$ & $\mathbf{5}$ & $\mathbf{6}$ & $\mathbf{7}$ & $\mathbf{8}$ & $\mathbf{9}$ & $\mathbf{1 0}$ & $\mathbf{1 1}$ & $\mathbf{1 2}$ \\
\hline $0 \mu \mathrm{M}$ & $0 \mu \mathrm{M}$ & $0.05 \mu \mathrm{M}$ & $0.05 \mu \mathrm{M}$ & $0.1 \mu \mathrm{M}$ & $0.1 \mu \mathrm{M}$ & $0.25 \mu \mathrm{M}$ & $0.25 \mu \mathrm{M}$ & $0.5 \mu \mathrm{M}$ & $0.5 \mu \mathrm{M}$ & $1 \mu \mathrm{M}$ & $1 \mu \mathrm{M}$ \\
$\mathrm{H}_{2} \mathrm{O}_{2}$ & $\mathrm{H}_{2} \mathrm{O}_{2}$ & $\mathrm{H}_{2} \mathrm{O}_{2}$ & $\mathrm{H}_{2} \mathrm{O}_{2}$ & $\mathrm{H}_{2} \mathrm{O}_{2}$ & $\mathrm{H}_{2} \mathrm{O}_{2}$ & $\mathrm{H}_{2} \mathrm{O}_{2}$ & $\mathrm{H}_{2} \mathrm{O}_{2}$ & $\mathrm{H}_{2} \mathrm{O}_{2}$ & $\mathrm{H}_{2} \mathrm{O}_{2}$ & $\mathrm{H}_{2} \mathrm{O}_{2}$ & $\mathrm{H}_{2} \mathrm{O}_{2}$
\end{tabular}

After the incubation period, working solution 1 from each well was transferred into a new reaction tube. From each tube, $100 \mu$ of solution were pipetted into three wells of the black 96-well plate, respectively. Luminescence was measured with a Mithras ${ }^{2}$ LB 943 plate reader (Berthold Technologies, Bad Wildbad, Germany) at RT with the following settings: fluorescence was measured at $540 \mathrm{~nm}$ excitation (filter F540) and $620 \mathrm{~nm}$ emission (filter Em600/10) for $0.1 \mathrm{sec}$ with $0.2 \mathrm{sec}$ delay and $40 \%$ of the lamp energy. Measurement values were processed with Microsoft excel and plotted with Graphpad Prism 7.

\subsection{Immunocytochemistry staining}

Expression of cellular proteins was shown with immunocytochemistry staining as described earlier by our group (Borchert et al., 2017; Haupt, 2018). In short, iPSCs or iPSC-CMs were cultured on glass cover slips, fixed with Histofix for $20 \mathrm{~min}$ at RT and blocked with $1 \% \mathrm{BSA} / \mathrm{PBS}$ overnight at $4^{\circ} \mathrm{C}$. When staining intracellular proteins, iPSCs or iPSC-CMs 
were permeabilized with $0.1 \%$ Triton $\mathrm{X}-100$ for $10 \mathrm{~min}$ at RT. All samples were incubated with the respective primary antibody overnight at $4^{\circ} \mathrm{C}$ in a humidified chamber. The following day, the glass cover slips were washed three times in PBS and then incubated with a corresponding secondary antibody for $1 \mathrm{~h}$ at $37^{\circ} \mathrm{C}$. Afterwards, the cover slips were washed three times in PBS and then the nuclei were stained with DAPI for $10 \mathrm{~min}$ at RT in the dark. Then, the cover slips were washed three times in PBS and once in Millipore water. Finally, the cover slips were mounted onto microscopy slides with Vectashield mounting medium and sealed with nail polish. Images were captured with a Zeiss Axio Observer.Z1 microscope and a Zeiss AxioCam MRm 1.4MP camera. Detailed information about the used antibodies is listed in Table 3-4.

\subsection{Cell size}

Cell size was determined with CASY cell counter, which allowed fast three-dimensional measuring of iPSC-CMs.

Ten days before the assay, 25,000 iPSC-CMs were seeded onto each well of a 12-well plate and cultured in cardio culture medium under standard conditions. On the day of the assay, brightfield images of each well were captured for reference. Then, iPSC-CMs were detached from the plate with trypsin/EDTA, diluted in CASY buffer and measured with the CASY device. The limits for detection were set to $14.99 \mu \mathrm{m}$ (minimum) and $45.02 \mu \mathrm{m}$ (maximum) according to the expected size range for iPSC-CMs. Each sample from an individual well was counted three times, and mean diameter and mean volume were displayed in excel files and plotted with GraphPad Prism 7. Our former group member Ahmed Wagdi automated parts of the analysis with Python scripts.

\subsection{Analysis of sarcomeric organization}

The myofilament structure was observed with a-actinin staining (see Immunocytochemistry staining). To assess its degree of organization, tubular structures in the images were emphasized with the "Tubeness" plugin of the FIJI software. Then we applied the fast Fourier transform algorithm (FFTA). After applying FFTA, the frequency domains were radially integrated with "Radial Profile Plot" from Paul Baggethun (as in Ball et al., 2015; Chlanda et al., 2016; Taffoni et al., 2019). These steps were performed by a macro for FIJI, which was written and extended by the former group members Thomas Borchert and Dr. Luis Haupt (Haupt, 2018). A Python script written by Ahmed Wagdi automated and accelerated the process. The resulting intensity profiles were analyzed with Lab Chart software (BD Instruments). The relative amplitude of the first peak positively correlates with the regularity of $\alpha$-actinin striations, and it was used as data points for sarcomeric regularity. 


\subsection{Statistical analysis}

Individual data points are depicted as dots, with boxes indicating the mean +/- standard error of the mean (SEM). Outliers were identified with ROUT (robust regression followed by outlier identification) (Motulsky and Brown, 2006), $Q=1$ with Prism (GraphPad). Statistical significance of data sets consisting of two groups was tested with a two-tailed Student's ttest. Data sets with more than two groups were tested with one-way analysis of variance (ANOVA) and multiple comparisons between treatments were corrected according to Tukey and multiple comparisons between patients were corrected according to Sidak. Data sets where two factors were influenced were tested with two-way ANOVA. Multiple comparisons of two-way ANOVAs were corrected according to Tukey or Sidak. Significances were indicated with ${ }^{*}=p<0.05 ;{ }^{* *}=p<0.01 ;{ }^{* * *}=p<0.001$. Exact $p$ values and confidence intervals are listed in the appendix. In all analyses, Prism versions 7 and 8 (GraphPad) were used.

\subsection{Software for representation of data}

Graphs were plotted with GraphPad Prism versions 7 and 8. Figure 4-1 and Figure 4-2 were created with DNADynamo. Visualization of sequencing data was performed with BioEdit (Figure 5-7 and Figure 5-13). Figure 2-2, Figure 2-3, and Figure 5-8 were designed on the BioRender.com platform. The arrangement of figures was done with CorelDRAW. 


\section{Results}

\subsection{Basic impact of DOX}

Other groups and we have shown that ACT can be modeled with iPSC-CMs. ACT-iPSC-CMs have shown a higher susceptibility to the damaging effects of DOX compared to controliPSC-CMs. The differences are manifested in higher ROS production, disruption of sarcomeric regularity, increased rates of apoptosis, and aberrant calcium handling (Burridge et al., 2016; Haupt, 2018).

\subsubsection{Resorption and retention of DOX in an ACT model}

In order to accurately describe the molecular mechanisms underlying the previously mentioned changes, it is important to understand the resorption of DOX. To date, there have been no publications addressing subcellular and time-dependent distributions of DOX in human cardiomyocytes. The initial aim of this study was to identify resorption rates, possible maximal resorption doses, retention, and subcellular distribution of the DOX in iPSC-CMs. Therefore, we used the previously characterized iPSC-CM cohort from a former study in our lab (Haupt, 2018). Briefly, five patients were selected from the RICOVER-60 trial, which investigated the outcome of anthracycline treatment of elderly B-cell lymphoma patients (Pfreundschuh et al., 2008). Three of the cancer patients had developed ACT and are assigned here as ACT patients. Two other patients, who had received similar accumulative doses of DOX, did not develop ACT and served as control patients. The three ACT patients carried homozygous or heterozygous SNPs in genes encoding subunits of Nox2. The two control patients had wild-type (wt) sequences at these positions. These SNPs have been linked to the occurrence of ACT (Wojnowski et al., 2005), and were also described in the RICOVER-60 trial (Reichwagen et al., 2015). The five selected patients donated skin biopsies that were used to extract skin fibroblasts. These were reprogrammed into iPSCs by introducing the Yamanaka factors with a Sendai virus. Characterized iPSCs were cultured in feeder-free culture and differentiated into iPSC-CMs as described previously (Borchert et al., 2017; Haupt, 2018). The resulting iPSC-CMs from ACT patients and iPSC-CMs from the controls showed patient-specific genetic backgrounds and were used in this study to reveal potential differences between the two groups regarding their reactions to DOX exposure.

iPSC-CMs of ACT patients and controls were treated with DOX for different periods or with various concentrations. Immediately after the respective treatment period, iPSC-CMs were lysed and the samples were processed for HPLC measurement. To address reported longterm damages caused by DOX (Middleman et al., 1971; Serrano et al., 1999), in one part of the study, iPSC-CMs were given time to recover from the treatment. Afterwards, the iPSCCMs were washed with PBS, harvested with acetonitrile and the amount of intracellular DOX was determined with HPLC. As a reference, DOX diluted in PBS was also injected, which resulted in a specific peak around $16 \mathrm{~min}$ in the fluorescent channel $(510 \mathrm{~nm}$ excitation/595 
$\mathrm{nm}$ emission). HPLC peak analysis was performed with Chromeleon 7 software and the area under the curve was measured for each peak.

\subsubsection{Concentration-dependent DOX resorption}

From previous studies in the lab, we know that DOX exposure leads to increased extra- and intracellular levels of ROS, aberrant calcium handling, sarcomeric irregularity and increased rates of apoptosis in iPSC-CMs from both groups, ACT patients and control patients (Haupt, 2018). Regarding ROS levels, sarcomeric regularity, and apoptosis, the observed detrimental effects were enhanced with increasing DOX concentrations (Haupt, 2018). Intracellular $\mathrm{H}_{2} \mathrm{O}_{2}$ levels in both groups doubled after treatment with $5 \mu \mathrm{M}$ DOX compared to treatment with $0.25 \mu \mathrm{M}$ DOX (Haupt, 2018). Apoptosis rates in both groups were about 1.5 times higher with $1 \mu \mathrm{M}$ DOX as compared to $0.5 \mu \mathrm{M}$ DOX when treated for $24 \mathrm{~h}$ (Haupt, 2018). After treatment for $72 \mathrm{~h}$, apoptosis rates in both groups were approximately three times higher with $1 \mu \mathrm{M}$ DOX as compared to $0.5 \mu \mathrm{M}$ DOX. Furthermore, the apoptosis rate in both groups tripled after $72 \mathrm{~h}$ with $1 \mu \mathrm{M}$ DOX, compared to $24 \mathrm{~h}$ with the same concentration (Haupt, 2018). These results showed that DOX-triggered effects in IPSC-CMs are dose and time dependent. However, it is still unknown whether DOX accumulates in iPSC-CMs and intracellular DOX levels depend on treatment time and concentration.
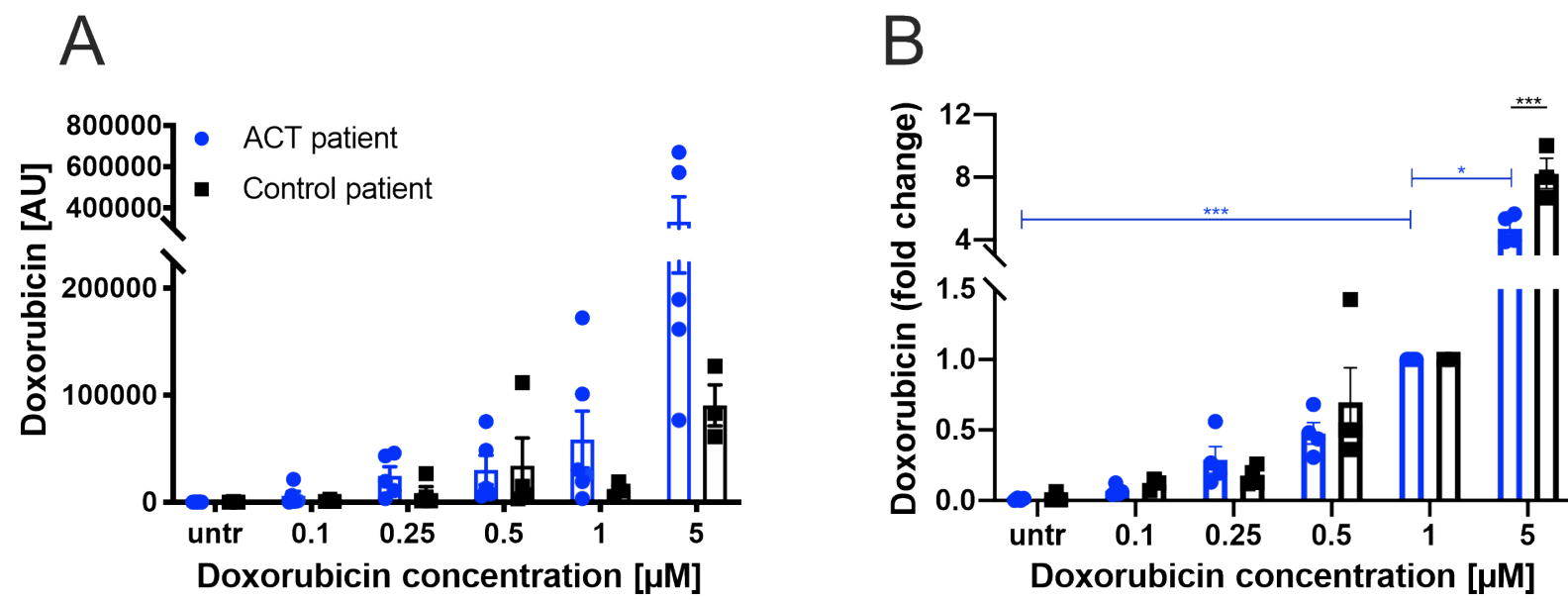

Figure 5-1: Intracellular DOX levels in iPSC-CMs increased with rising DOX concentrations.

The intracellular DOX concentration increased with higher treatment concentrations of DOX in the culture medium. A: Intracellular DOX values increased with higher DOX concentrations in both ACT patient (blue columns) and control (black columns) groups. At concentrations of $1 \mu \mathrm{M}$ and $5 \mu \mathrm{M}$ DOX, the intracellular DOX content was higher in ACT-iPSC-CMs compared to control iPSC-CMs. B: When values were normalized to the $1 \mu \mathrm{M}$ treatment conditions, intracellular DOX levels in ACT-iPSC-CMs were significantly higher at $1 \mu \mathrm{M}$ DOX compared to untreated. Untr=untreated. $\mathrm{AU}=$ arbitrary unit. $\mathrm{n}$ = one independent iPSC-CM differentiation experiment. ACT patient: $n=$ five differentiations from four iPSC lines from two patients. Control patient: $n=$ three differentiations from three iPSC lines from two patients. Statistical analysis was performed with two-way ANOVA or mixed-effects analysis. $\mathrm{P}$ values are presented as followed: $\mathrm{p}>0.05=* ; \mathrm{p}>0.01=* * ; \mathrm{p}>0.005=* * *$. Bars indicate mean values +/- SEM. 
To determine the resorption of DOX, iPSC-CMs were challenged with various DOX concentrations from $0.1 \mu \mathrm{M}$ to $5 \mu \mathrm{M}$ DOX in the culture medium for $24 \mathrm{~h}$ at standard cell culture conditions $\left(37^{\circ} \mathrm{C}, 5 \% \mathrm{CO}_{2}\right)$. The results show that intracellular DOX concentrations increased with rising DOX treatment concentrations (Figure 5-1A). Notably, the increase of intracellular DOX was highly associated with DOX treatment concentrations, e.g. $1 \mu \mathrm{M}$ DOX in ACT-iPSC-CMs resulted on average in an area under the curve value of 58,598 , which is close to double the value of $0.5 \mu \mathrm{M}(30,120)$, and approximately a fifth of the value of $5 \mu \mathrm{M}$ $(334,208)$. DOX was detectable at the lowest tested media concentration of $100 \mathrm{nM}$ DOX and did not reach a plateau in the tested range up to $5 \mu \mathrm{M}$ (Figure 5-1A). Although not significant, DOX levels were higher in ACT-iPSC-CMs compared to control-iPSC-CMs under nearly all tested concentrations. When normalized to $1 \mu \mathrm{M}$ DOX, the intracellular increase in DOX concentration reached significance at $1 \mu \mathrm{M}$ DOX in the ACT patient group compared to the untreated iPSCs (Figure 5-1B). Comparing normalized values, intracellular DOX concentrations were significantly higher in control patients than in ACT patients at $5 \mu \mathrm{M}$ DOX. While the absolute DOX levels were higher in ACT-iPSC-CMs compared to controliPSC-CMs, the normalized values show that the correlation to treatment concentrations is prominent in both groups (Figure 5-1B).

\subsubsection{Time-dependent DOX resorption}

To estimate the kinetics of DOX resorption, iPSC-CMs from both groups were challenged with $1 \mu \mathrm{M}$ DOX and cultured in standard conditions for time periods between 30 min to $72 \mathrm{~h}$. Already after $30 \mathrm{~min}$, DOX was detectable in iPSC-CMs of both groups (Figure 5-2A). After $2 \mathrm{~h}$ and $6 \mathrm{~h}$ of treatment, DOX values were significantly higher in the control group compared to untreated iPSC-CMs, whereas the absolute DOX values were higher in the ACT patient group compared to controls. The intracellular amount of DOX increased with treatment time and reached its peak after $48 \mathrm{~h}$. After $72 \mathrm{~h}$ of treatment, the values decreased and were comparable to the values at the $24 \mathrm{~h}$ time point (Figure 5-2B).
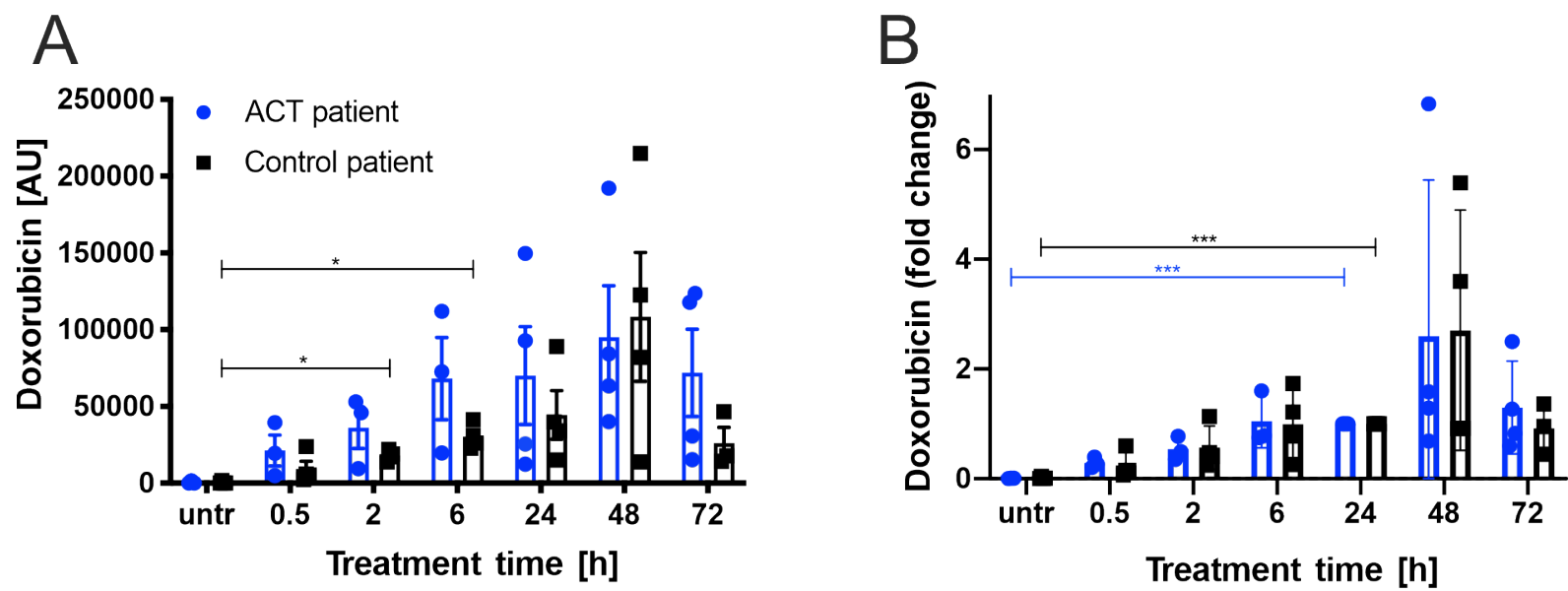

Figure 5-2: Intracellular DOX levels in iPSC-CMs rose with increasing DOX treatment time. 
Intracellular DOX concentration increased with longer treatment time of $1 \mu \mathrm{M}$ DOX in the culture medium. A: Intracellular DOX levels increased with higher treatment time up to $48 \mathrm{~h}$ in ACT- (blue columns) and control-iPSC-CMs (black columns), but decreased after $72 \mathrm{~h}$ DOX treatment. B: When normalized to the $24 \mathrm{~h}$ time point, the results showed significantly higher DOX levels in both groups at $24 \mathrm{~h}$ compared to untreated controls. $\mathrm{AU}=$ arbitrary unit. $\mathrm{n}=$ one independent $\mathrm{iPSC}$-cardiomyocyte differentiation experiment. ACT patients: $n=$ four differentiations from two cell lines from one patient. Control patients: $n=$ four differentiations from two cell lines from one patient. Statistical analysis was performed with two-way ANOVA or mixed-effects analysis. $P$ values are represented as followed: $p>0.05=* ; p>0.01=* * ; p>0.005=* * *$. Bars indicate mean values $+/-$ SEM.

Normalization to the $24 \mathrm{~h}$ DOX treatment time point shows that the kinetics of intracellular DOX resorption was comparable in ACT- and control-iPSC-CMs (Figure 5-2B). DOX levels at the $24 \mathrm{~h}$ time point were significantly higher compared to untreated iPSC-CMs in both ACTand control-iPSC-CMs (Figure 5-2B).

\subsubsection{DOX content after washout}

Chronic ACT can manifest itself many years or even decades after cancer treatment (reviewed by Minotti et al., 2004). It is conceivable that a small amount of DOX remains in cardiomyocytes over a long period of time; hence, continuously causing damage that becomes apparent in the long term. To test this hypothesis, iPSC-CMs were treated with $1 \mu \mathrm{M}$ DOX for $24 \mathrm{~h}$ and cultured under standard conditions for three days and seven days (Figure 5-3A). After the treatment period (time point 0 ), the medium was removed and the iPSC-CMs were cultured in regular cardio culture medium without DOX. For iPSC-CMs that were harvested at time point seven, cardio culture medium was changed at time point three (Figure 5-3A).
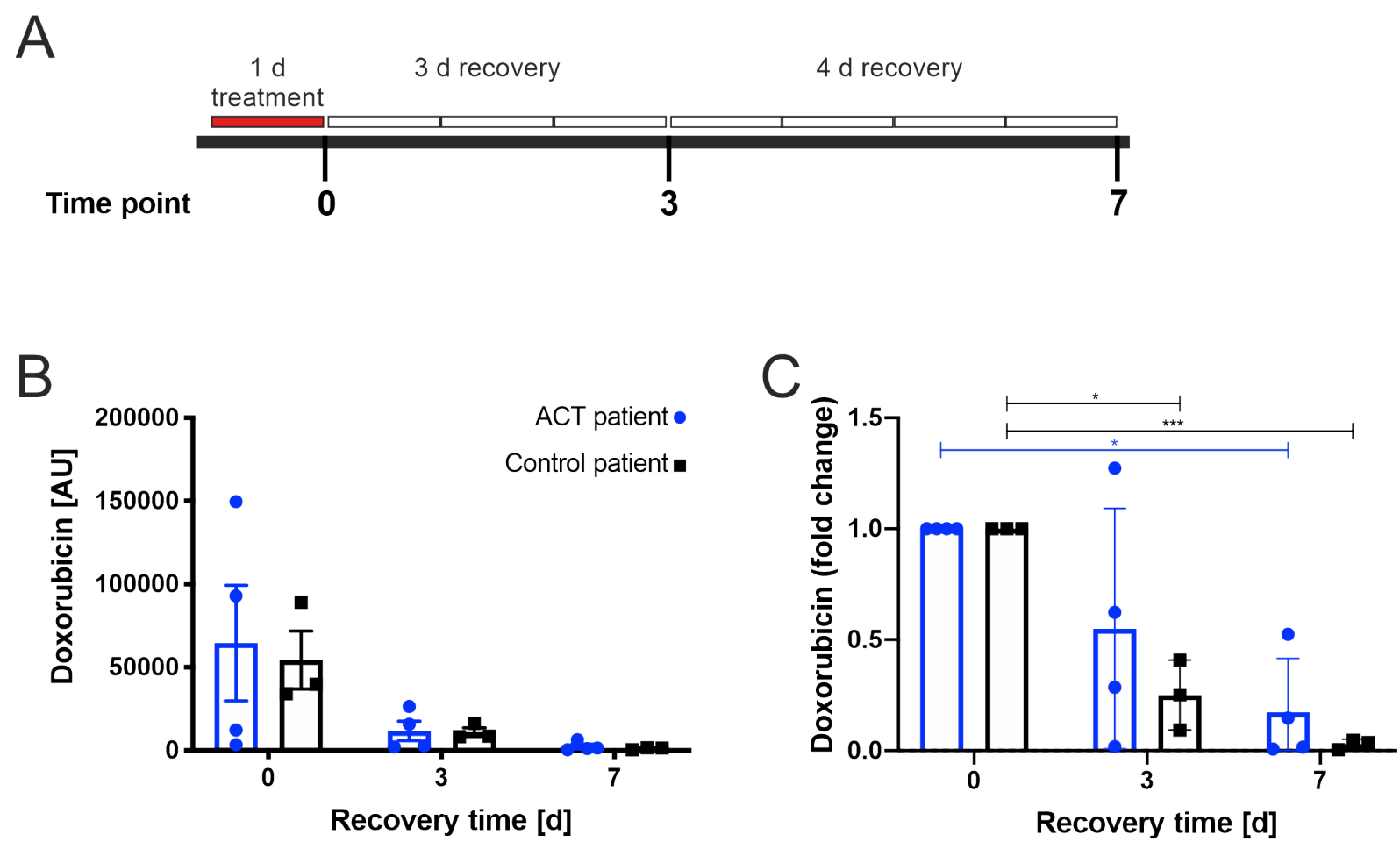

Figure 5-3: Decrease of intracellular DOX in iPSC-CMs after recovery period. 
Intracellular DOX levels decreased after one day of recovery and reached the detection threshold after seven days of recovery. A: The time line visualizes treatment and recovery periods. iPSC-CMs were treated with $1 \mu \mathrm{M}$ DOX for one day. iPSC-CMs were harvested, or medium was changed at time point zero, after three days, or after seven days after DOX treatment. B: The decrease of intracellular DOX levels after recovery was comparable between ACT-iPSC-CMs (blue columns) and control-iPSC$\mathrm{CMs}$ (black columns). C: When normalized to time point zero (one day of DOX treatment, $\mathrm{O} \mathrm{h}$ recovery), control-iPSC-CMs showed a significant decrease after three days. In ACT-iPSC-CMs, DOX was significantly decreased after seven days of recovery. $A U=$ arbitrary unit. $n=$ one independent iPSC-cardiomyocyte differentiation experiment. ACT patient: $n=$ four differentiations from two iPSC lines from one patient. Control patient: $n=$ three differentiations from two iPSC lines from one patient. Statistical analysis was performed with two-way ANOVA or mixed-effects analysis. $P$ values were represented as followed: $p>0.05=* ; p>0.01 *^{* *} ; p>0.005=* * *$. Bars indicate mean values + /- SEM.

Three days of recovery resulted in a significant decrease of intracellular DOX of approximately $75 \%$ in both the ACT- and control group (Figure 5-3B). After seven days of recovery, the abundance of DOX was below or close to the detection limit in both groups (Figure 5-3B). Notably, when values were normalized to time point 0 , the decrease of intracellular DOX became significant after three days in the control group (Figure 5-3C), whereas a similar (25\% in control vs. $17.3 \%$ in ACT) significant decrease in intracellular DOX was reached in ACT-iPSC-CMs after seven days of recovery (Figure 5-3C).

These results indicate that a significant DOX amount did not remain in the iPSC-CMs for extended periods and that the washout was faster in iPSC-CMs from control patients than in iPSC-CMs from ACT patients. This supports the 'hit-and-run' model of DOX-induced damage, where DOX induces changes that continue to affect the cell past the period when DOX is present (Vesell and Passananti, 1971; Cowgill et al., 2019). In line with that, our group has shown that DOX treatment leads to enhanced extracellular $\mathrm{H}_{2} \mathrm{O}_{2}$ levels for up to three weeks after treatment (Haupt, 2018). Immediately after $24 \mathrm{~h}$ of $0.25 \mu \mathrm{M}$ DOX treatment, $\mathrm{H}_{2} \mathrm{O}_{2}$ production in ACT-iPSC-CMs and control-iPSC-CMs was increased by twofold (Haupt, 2018). One week after DOX treatment, $\mathrm{H}_{2} \mathrm{O}_{2}$ levels were increased 3- to 4-fold and three weeks after DOX treatment, the increase in $\mathrm{H}_{2} \mathrm{O}_{2}$ production was still two-fold compared to untreated iPSC-CMs (Haupt, 2018). Taken together, we have shown that DOX is cleared from iPSC-CMs within one week, while DOX-induced effects, such as enhanced $\mathrm{H}_{2} \mathrm{O}_{2}$ production, remain for several weeks.

\subsubsection{Subcellular distribution of DOX}

DOX is described to bind to the mitochondrial phospholipid cardiolipin, hence, we expected the drug to accumulate prevalently in close proximity to mitochondria rather than in other cell compartments (Nicolay et al., 1984; Goormaghtigh et al., 1990; Aryal and Rao, 2016). Using differential ultracentrifugation, we separated the mitochondrial and nucleus fraction, termed here mito fraction, from the membrane fraction and cytoplasm (Laurindo et al., 2002). The amount of DOX per fraction was then determined with HPLC. 
A Total doxorubicin

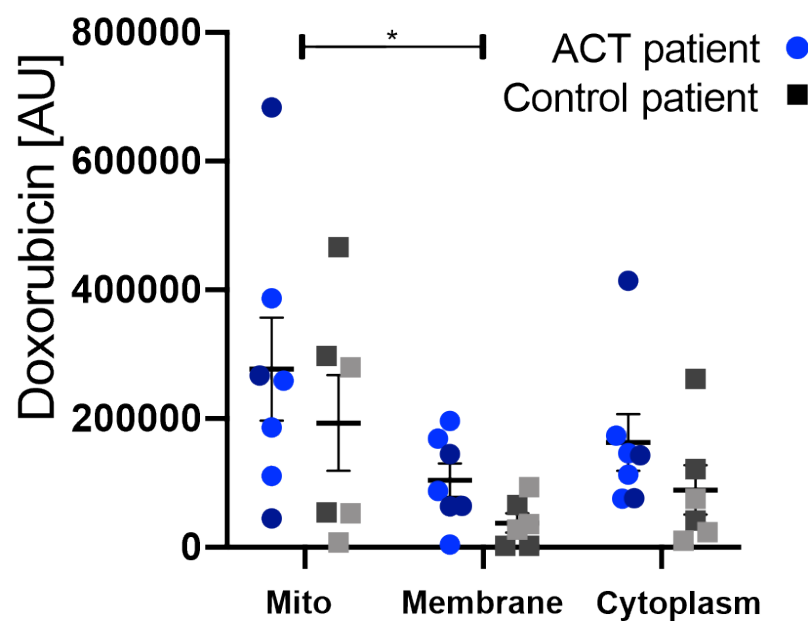

B
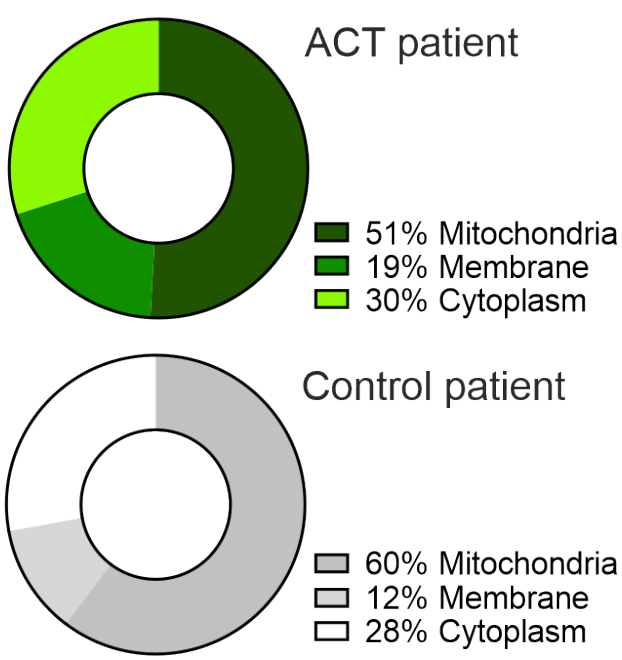

Figure 5-4: Subcellular distribution of DOX in iPSC-CMs after treatment with $1 \mu \mathrm{M}$ DOX for $24 \mathrm{~h}$.

Three distinct cell fractions were analyzed with HPLC. A: Measuring total DOX content, the mito fraction showed the highest DOX levels among the three fractions. The DOX levels in the mito fractions were significantly higher than in the membrane fractions. B: The ring charts illustrate the distribution of DOX over the three fractions in the ACT- and control group. In ACT-iPSC-CMs, $51 \%$ of DOX was detected in the mito fraction. In control-iPSC-CMs, $60 \%$ of DOX was found in the mito fraction. $\mathrm{AU}=$ arbitrary unit. Statistical analysis was performed with two-way ANOVA. $\mathrm{n}=$ seven independent differentiations from two cell lines from two patients (ACT-iPSC-CMs), $n=$ six independent differentiations from two cell lines from two patients (control-iPSC-CMs). For A, different shades of blue and grey indicate data points from different patients. The $p$ value was represented as followed: $\mathrm{p} 0.04=*$. Horizontal bars indicate the mean $+/$ - SEM.

Mean DOX levels were higher in the mito fraction compared to membrane or cytoplasmic fraction in both ACT- and control-iPSC-CMs (Figure 5-4A). DOX levels in the mito fractions were significantly higher than in the membrane fractions (Figure 5-4A, appendix). For all tested fractions (mito, membranes, cytoplasm), the mean DOX levels were higher in ACTiPSC-CMs compared to control-iPSC-CMs. Focusing on the distribution of DOX among the three fractions, in both groups the highest percentage of DOX was detected in the mito fraction, followed by the cytoplasm, and the least in the membrane fraction (Figure 5-4A). In detail, in the ACT patient group, $51 \%$ of DOX was detected in the mito fraction, $30 \%$ in the cytoplasm and $19 \%$ in the membrane fraction (Figure 5-4B). A similar DOX distribution could be observed in the control-iPSC-CMs with $60 \%$ of the whole amount of DOX in the mito, $28 \%$ in the cytoplasm and $12 \%$ in the membrane fraction (Figure 5-4B). Comparing these percentages between the groups, a smaller mitochondrial percentage was observed in the ACT patient group $(51 \%)$ than in the control patient group $(60 \%)$. Furthermore, in both groups less than $20 \%$ of DOX was detected in the membrane fraction $(19 \%$ in the ACT patient group and $12 \%$ in the control patient group). It should be noted that the membranefraction pellets were considerably smaller in size and volume than the pellets containing the mito fractions and the cytoplasm. To achieve a more informative measure of DOX 
distribution, initial HPLC values were normalized to respective protein levels of the individual cell fractions.

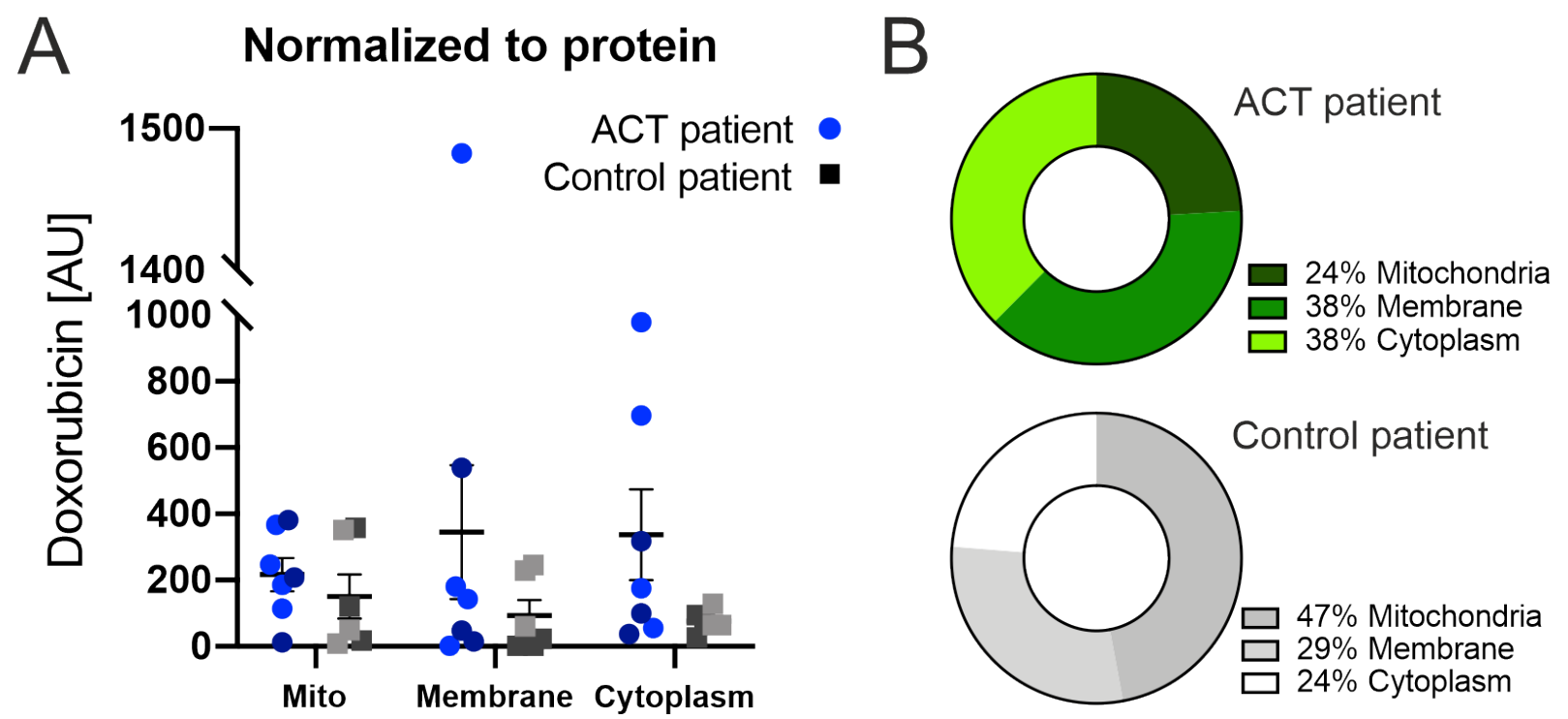

Figure 5-5: DOX is distributed relatively evenly throughout fractions of DOX-treated iPSC-CMs.

A: Fractions of ACT-iPSC-CMs contained higher amounts of DOX compared to control-iPSC-CMs. B: In the ACT-iPSC-CMs, DOX was distributed evenly between the membrane and the cytosolic fraction (both $38 \%$ ), with less DOX in the mito fraction (24\%). In the control-iPSC-CMs nearly half of DOX was detected in the mito fraction (47\%). The rest of the DOX was distributed almost equally between the membrane (29\%) and the cytosolic fraction (24\%). AU= arbitrary unit. Statistical analysis was performed with two-way ANOVA. $n=7$ independent differentiations from 2 cell lines from 2 patients (ACT patient group), $n=6$ independent differentiations from 2 cell lines from 2 patients (Control patient group). Different shades of blue and grey indicate data points from different patients (A). Horizontal bars indicate the mean +/- SEM.

When normalized to the protein content, DOX distribution occurred relatively evenly throughout the fractions. Higher levels of DOX were detected in ACT patients compared to control patients, with an accumulative value of 898 [AU] in ACT-iPSC-CMs compared to 319 $[A U]$ in control-iPSC-CMs (Figure 5-5A). In each of the three fractions, the DOX value was higher in ACT-iPSC-CMs than in control-iPSC-CMs. In the mito fraction, the values were 217 in ACT vs. 151 in control; in the membrane fraction 344 in ACT vs 94 in control; and in the cytoplasmic fraction 337 in ACT vs 76 in control (Figure 5-5A). In the ACT patient group, DOX was distributed evenly between the membrane and the cytosolic fraction (both $38 \%$ ), with less DOX in the mito fraction (24\%). In the control patient group, nearly half of the total amount of DOX was detected in the mito fraction (47\%). DOX levels in the membrane and cytosolic fractions of the control patient group were similar $(29 \%$ in membrane fraction and $24 \%$ in the cytoplasm). While in the control patient group a high level of intracellular DOX was assigned to the mito fraction $(47 \%)$, the mean percentage of this fraction was lower in the ACT patient group (24\%) (Figure 5-5B). Overall, DOX levels in each fraction were higher 
in ACT-iPSC-CMs compared to control-iPSC-CMs. In conclusion, intracellular DOX concentrations differed between ACT-iPSC-CMs and control-iPSC-CMs.

\subsection{Effects of DOX treatment on mRNA translation}

Chronic DOX treatment can have a profound impact on the gene expression profile of cardiac cells (Berthiaume and Wallace, 2007; Holmgren et al., 2015, 2018; Li et al., 2019). Our group has previously found that a set of the investigated genes, including genes encoding for proteins that compose sarcomeric structure or act in calcium homeostasis, were down-regulated after DOX treatment (Haupt, 2018). Therefore, we aimed to determine to what extent DOX treatment would have a global effect on mRNA translation in an iPSC-CM ACT model. To investigate this question, we applied the SUnSET approach to detect potential changes in mRNA translation (Schmidt et al., 2009) and incubated DOX-treated iPSC-CMs and untreated iPSC-CMs with $1 \mu \mathrm{M}$ puromycin for $20 \mathrm{~min}$ at $37^{\circ} \mathrm{C}$ prior to lysis. Puromycin content in iPSC-CMs was detected with an anti-puromycin antibody and Western blotting. 


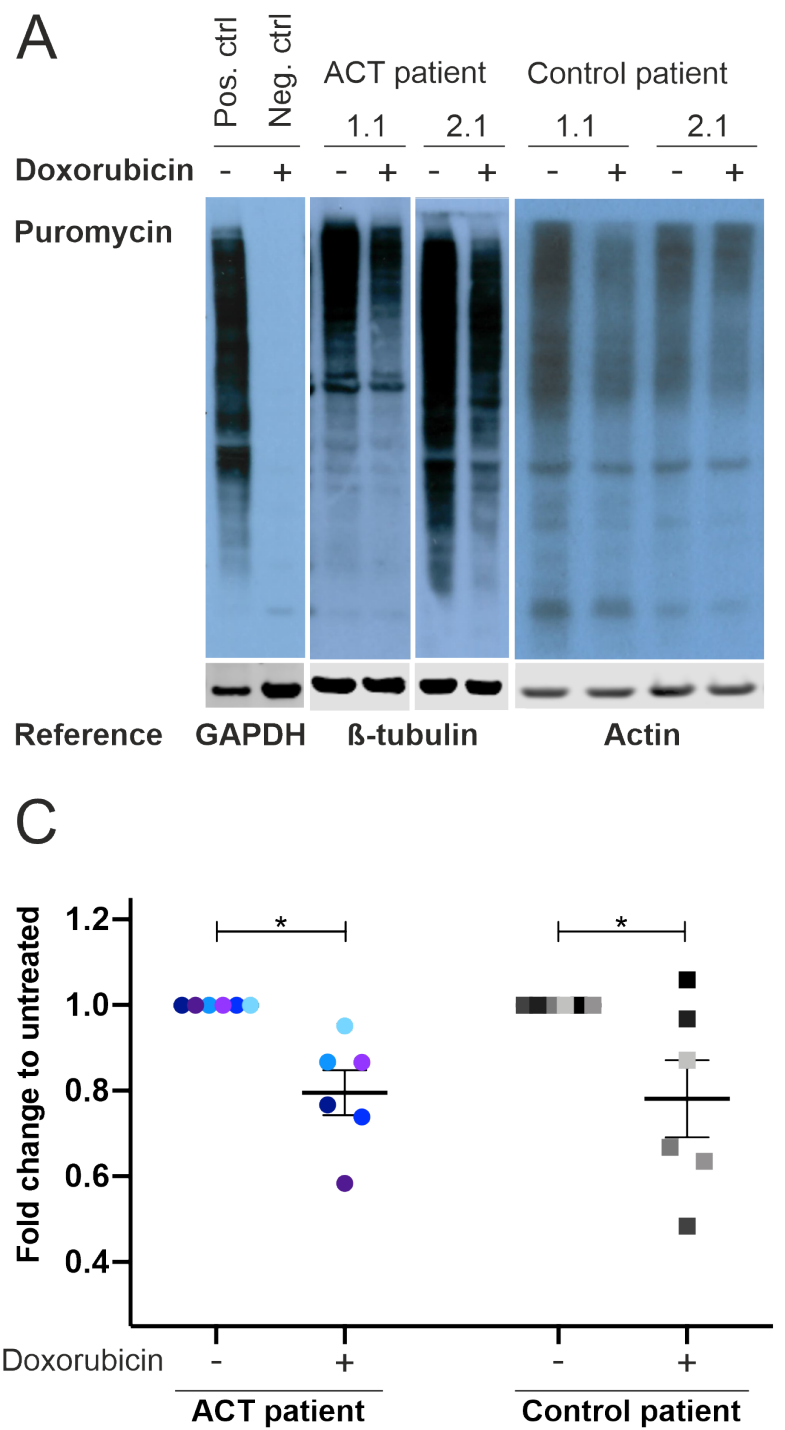

\section{B}

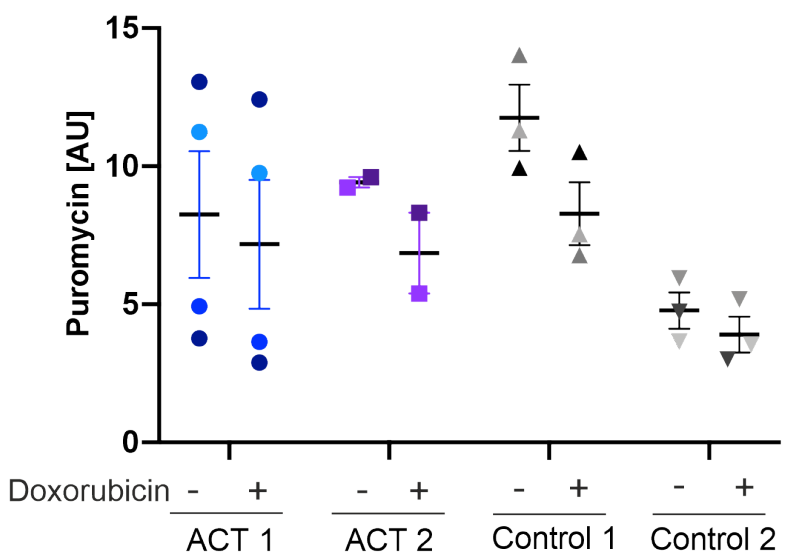

D

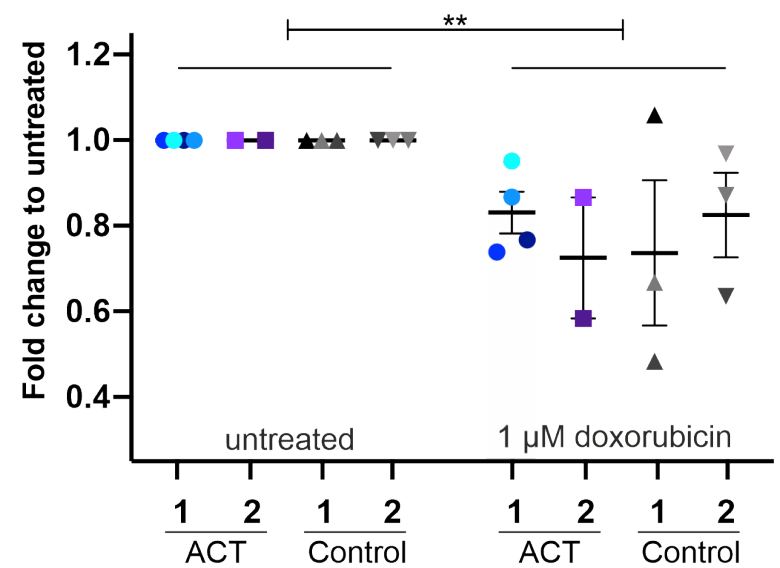

Figure 5-6: Global mRNA translation was decreased in iPSC-CMs after treatment with $1 \mu \mathrm{M}$ DOX for $24 \mathrm{~h}$.

mRNA translation was investigated with Western blot after puromycin treatment. Abundance of puromycin positively correlated with translation rates. A: Representative images of puromycin abundance after Western blotting. Positive control (Pos ctrl): Lysate from H9c2 human cardiomyoblast cell line incubated with $1 \mu \mathrm{M}$ puromycin for $30 \mathrm{~min}$. Negative control (Neg ctrl): lysate from DOX-treated control patient 2 iPSC-CMs without puromycin. As reference proteins GAPDH (left), $\beta$-tubulin (center), and actin (right) were used. B: Signal intensities per patient with and without DOX treatment. Values did not differ significantly between any cell lines. C: DOX treatment significantly reduced puromycin levels in all tested iPSC-CMs from both ACT-iPSC-CMs and controliPSC-CMs. The reduction was similar between the groups. D: Fold change of puromycin intensities per iPSC line with DOX treatment normalized to matched untreated values. DOX treatment had a significant negative impact on puromycin levels. $A U=$ arbitrary unit. Statistical analysis was performed with two-way ANOVA (B, D) and unpaired t-test $(\mathbf{C}) . n=$ signal intensities from one lane. Each lane is one independent differentiation. In total: six independent differentiations from two cell lines from two patients (respectively per group). $P$ values were represented as followed: $p>0.05=*$; $p>0.01=* *$. Horizontal bars indicate the mean $+/$ SEM.

Western blots of DOX-treated iPSC-CMs showed lower signals for puromycin compared to untreated iPSC-CMs from the same group. As a negative control, we used DOX-treated 
iPSC-CMs from ACT patient 2 without puromycin treatment and found one faint unspecific band (Figure 5-6A), while the positive control (internal control: lysate from H9c2 cells treated with $1 \mu \mathrm{M}$ puromycin for $30 \mathrm{~min}$ ) showed a strong signal. All quantification values came from two blots, in which actin was used as reference (Figure 5-6B-D). The representative blots in Figure $5-6 \mathrm{~A}$ are partially from earlier blots, where $\beta$-tubulin or GAPDH were used as reference. Quantified signal intensities confirmed a reduction in signal after DOX treatment, which was consistent throughout all tested cell lines (Figure 5-6B). Signal intensity and reduction of signal were in a similar range across all cell lines and did not differ significantly. Overall, DOX treatment led to a significant decrease of mRNA translation (Figure 5-6C). The decrease in translation after DOX treatment was significant in both ACT- and control-iPSCCMs (Figure 5-6D). There was no significant difference in mean signal reduction between the ACT patient and control patient group (Figure 5-6D), indicating that the DOX-induced decrease in translational processes is similar in ACT- and control-iPSC-CMs.

\subsection{Correcting a SNP with CRISPR/Cas9 gene editing} ACT patients and controls were selected according to the presence or absence of specific SNPs in genes that encode for subunits of the NADHPH oxidase. In this study, we focused on the SNP rs13058338 in the RAC2 gene (see 2.1.3). This genetic variant was described in clinical studies on the role of this SNP in ACT (Wojnowski et al., 2005; Reichwagen et al., 2015; Vulsteke et al., 2015; Leong et al., 2017). Table 5-1 presents an overview of characteristics of rs 13058338 in the RAC2 gene and shows the genotypes of the ACT cell lines in our group. A SNP-corrected ACT-iPSC line and its comparison to native iPSC-CMs from both ACT as well as control patients may reveal whether this polymorphism in the $R A C 2$ gene is mechanistically linked to ACT.

There are several approaches that could be used to investigate the impact of the described SNP on ACT development. First, one could test the activity of activated downstream complexes. In the case of ACT, Nox2 is the most promising downstream protein complex. Its activity can be either tested by complex assembly at the cell membrane or by direct measurement of its main product, superoxide. The low endogenous abundance of the Nox2 subunits p47phox and gp91phox in the iPSC-CMs made it difficult to precisely determine protein levels and their assembly by means of Western blotting. Instead, we conducted superoxide measurements of iPSC-CM membrane fractions with the ROS-sensing probe DHE. Assessment of the functional properties with the DHE probe was hampered by crossreactions with DOX (data not shown), and therefore was unable to produce reliable data. In another approach to assess the importance of the described SNP in the development of ACT, we aimed to correct the SNP in an ACT-iPSC line by reverting the polymorphism to the wt sequence. Then, functional assays, such as measurement of ROS production and calcium 
homeostasis, in the corrected iPSC-CMs may give hints whether these cells behave more like iPSC-CMs from the ACT patient group or from the control group.

Table 5-1: Overview of the single-nucleotide polymorphism rs13058338 in the RAC2 gene.

\begin{tabular}{|c|c|c|c|c|c|c|c|c|c|c|}
\hline \multirow[t]{2}{*}{ Gene } & \multirow[t]{2}{*}{ SNP } & \multirow[t]{2}{*}{ Genotype } & \multirow{2}{*}{$\begin{array}{l}\text { Occurrence } \\
\text { in general } \\
\text { population }\end{array}$} & \multirow[t]{2}{*}{$\begin{array}{l}\text { Nucleotide } \\
\text { exchange }\end{array}$} & \multirow[t]{2}{*}{$\begin{array}{l}\text { Effect of } \\
\text { SNP }\end{array}$} & \multicolumn{5}{|c|}{$\begin{array}{c}\text { Genotype of ACT cohort cell } \\
\text { lines }\end{array}$} \\
\hline & & & & & & $\begin{array}{l}\text { AC } \\
\text { T } 1\end{array}$ & $\begin{array}{l}\text { AC } \\
\text { T2 }\end{array}$ & $\begin{array}{l}\text { AC } \\
\text { T 3 }\end{array}$ & $\begin{array}{l}\text { Ctr } \\
11\end{array}$ & $\begin{array}{l}\text { Ctr } \\
12\end{array}$ \\
\hline Rac2 & $\begin{array}{c}\text { rs1305 } \\
8338\end{array}$ & $\begin{array}{l}T T=w t \\
A T / A A\end{array}$ & -15\% & $\begin{array}{l}\text { GRCh38 (hg38): } \\
\text { g.37236730T>A }\end{array}$ & $\begin{array}{l}\text { Unknown; } \\
\text { SNP in } \\
\text { intronic } \\
\text { region }\end{array}$ & AA & AT & AT & TT & TT \\
\hline
\end{tabular}

\subsubsection{SNP confirmation and primer validation}

Before attempting to correct the SNP in an ACT-iPSC line, we aimed to confirm again the presence of this aberration in the iPSC lines. Therefore, iPSCs from ACT patient 1 cell line 10 (ACT 1.10), ACT patient 2 cell line 14 (ACT 2.14), as well as control patient 1 cell line 2 (ACT K 1.2), and control patient 2 cell line 9 (ACT K 2.9) were collected, their gDNA extracted, amplified by PCR, and finally sequenced.

A

ACT 1.10

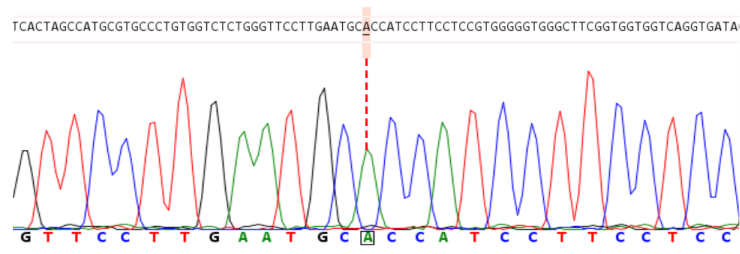

AA

C

ACT K 1.2

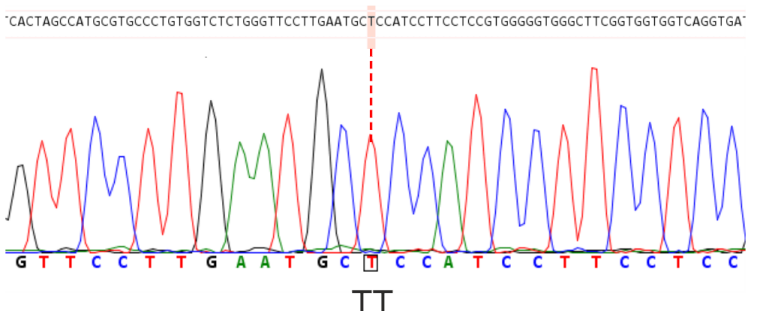

B

ACT 2.14

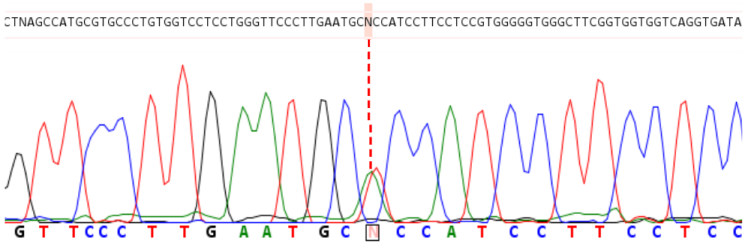

AT

$\mathrm{D}$

ACT K 2.9

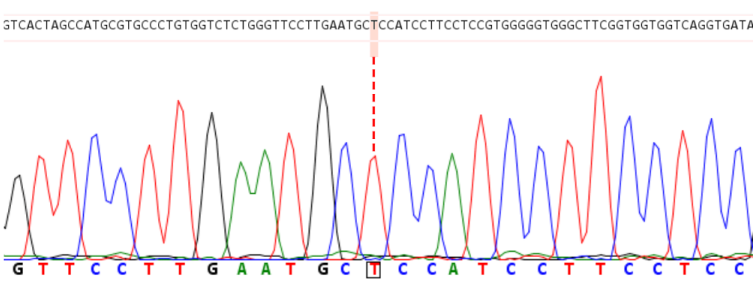

TT

Figure 5-7: Sanger sequencing results for SNP rs13058338.

Two ACT-iPSC lines and two control iPSC lines were tested with Sanger sequencing in the RAC2 gene for the SNP rs13058338. A: In ACT patient 1, the homozygous mutation was confirmed. B: ACT patient 2 carried a heterozygous mutation at that location. C, D: Both control-iPSC lines expressed the wild-type sequence.

Sanger sequencing (carried out by Microsynth AG, Balgach, Switzerland) confirmed that iPSCs of ACT patient 1 carried a homozygous mutation in RAC2 (SNP rs13058338) with AA instead of TT, and ACT patient 2 carried a heterozygous mutation (AT; Figure 5-7). iPSCs of 
control patient 1 and control patient 2 showed a wt sequence at these positions (TT; Figure $5-7)$.

\subsubsection{Design of HDR templates and guide RNAs}

The aim of genome editing was to correct the SNP in RAC2 to the respective wt sequence. To specifically target RAC2, a gRNA and an HDR template were designed (Figure 5-8). Two different online tools were used to design the gRNA sequence and to select the most promising outputs. Initially, the 'Custom Alt-R CRISPR-Cas9 guide RNA' tool was used, which is provided by Integrated DNA Technologies (IDT; Coralville, IA, USA). As a second parameter, CRISPOR.org was used (Concordet and Haeussler, 2018). The binding sequence for the gRNA, the sequence for the HDR template, and the predicted scores from both tools are depicted in Figure 5-8. The selected gRNA was chosen because it cuts close to the SNP (five base pairs [bps] upstream) and had a low off-target score. The off-target score includes potential mismatches in which the gRNA binds to sequences that differ in one or more bps from the gRNA sequence. For zero and one mismatches, the chosen gRNA is predicted to bind to no sequences other than the target sequence. For any two mismatches, the gRNA is predicted to potentially bind to four off-target sequences and for three mismatches to 35 . This favorable off-target score and the proximity of the cutting site to the SNP determined the choice of gRNA and the derived HDR template.

A

Sequence around Single nucleotide polymorphism

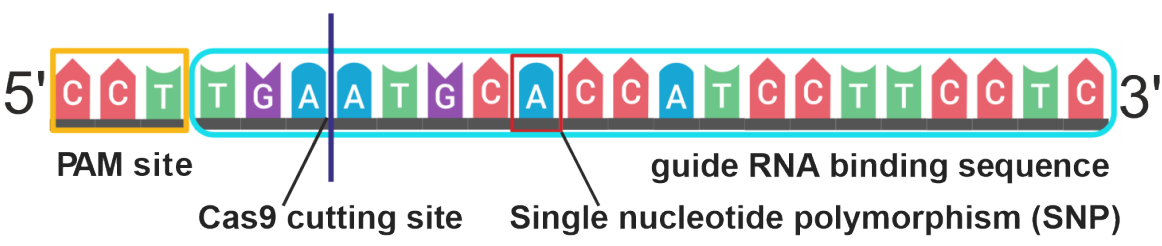

Off target/mismatch predictions:

IDT: 0

CRISPOR: 0-0-4-35-261 for 0-1-2-3-4 mismatches

B

HDR template

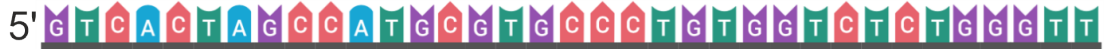

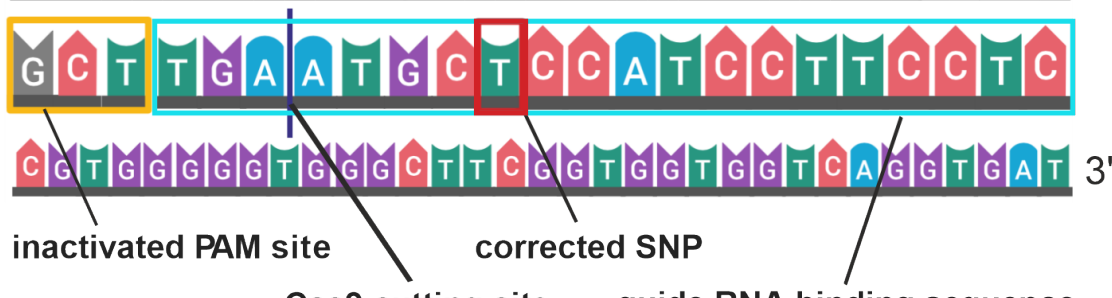

Cas9 cutting site guide RNA binding sequence

Figure 5-8: Nucleotide sequences around the SNP with the gRNA binding sequence and the HDR template. 
A: The gRNA binding sequence contained the SNP (red box), which was located seven nucleotides downstream of the next PAM site (yellow box). The Cas9 cutting site was five base pairs upstream of the SNP. Prediction scores of two independent databases calculated low probabilities for mismatches. A mismatch of ' 2 ' means that the guide RNA is expected to bind to a sequence that differs in two bases from the intended binding sequence. The CRISPOR score indicates that in this case, there are four off-site targets. For any three mismatches, there are 35 targets, etc. B: The HDR template contained 45 nucleotides on each side of the SNP. The mutant nucleotide itself was swapped with the wild-type nucleotide ( $T$ for A, red box). To limit Cas9 activity, the PAM site was destroyed with a synonymous mutation ( $G$ for $C$, yellow box). Since the PAM site is located in an intronic region, no coding triplets are defined. PAM: Protospacer adjacent motif. SNP: Singlenucleotide polymorphism.

\subsection{3. iPSC electroporation and culture with HDR enhancer}

The CRISPR-RNA-Cas9 complex was transfected into iPSCs using electroporation. Two days prior to transfection, iPSCs from the ACT 1.10 line were seeded out and cultured in StemFlex medium under standard conditions (Figure 5-9). On day zero, iPSCs were electroporated with the CRISPR-RNA-Cas9 complex, gRNA, and the Rac2-specific HDR template, which resulted in an iPSC line named ACT 1.10_R. Cas9-induced DSBs can be resolved through both NHEJ and HDR, although the predominant repair mechanism is NHEJ (Frit et al., 2014). The integration of the HDR template relies on the HDR repair pathway. To increase the rate of DSB repair through HDR, we tested the small molecule reagent 'Alt-R HDR enhancer' (IDT, Coralville, IA, USA), which has been described by the manufacturer to enhance gene editing by $50 \%-400 \%$ in different human cell lines (Schubert et al., 2018). The HDR enhancer was added directly to the culture medium after electroporation for $24 \mathrm{~h}$ at two different concentrations (Figure 5-9). Since the HDR enhancer is dissolved in DMSO, the highest recommended dose is $30 \mu \mathrm{M}$ to avoid cytotoxicity. We used $20 \mu \mathrm{M}$ and $30 \mu \mathrm{M}$, respectively, and cultured an additional well of electroporated iPSCs without HDR enhancer. After one day, the HDR enhancer was removed and the iPSCs were cultured in StemFlex medium for additional five days (Figure 5-9).

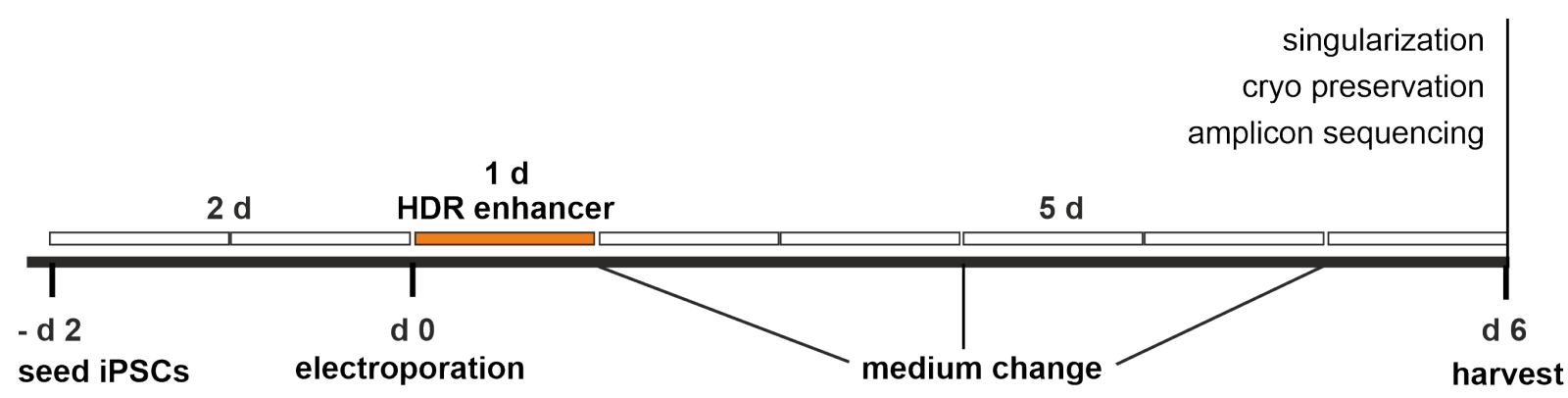

Figure 5-9: Time line of CRISPR/Cas9 gene editing with electroporation.

iPSCs were cultured for two days prior to electroporation. Six days after electroporation, iPSCs were harvested for further processing.

On day one after electroporation, iPSCs were examined for growth, density, and degree of cell debris in the culture medium. iPSCs of the non-transfected control were attached to the plate (Figure 5-10, left). Transfected iPSCs showed signs of distress, such as detaching from 
the plate (Figure 5-10, center left), where cells appear as bright, round balls. iPSCs which were treated with $20 \mu \mathrm{M}$ HDR enhancer showed even lower cell density (Figure 5-10, center right) and visibly more debris in the culture medium that was even more prominent with an HDR enhancer concentration of $30 \mu \mathrm{M}$ (Figure 5-10, right). Overall, addition of HDR enhancer led to more cell debris, indicating elevated cell death and resulted in a lower number of cells. More important than cell numbers was the rate of successful editing, which was estimated with Amplicon sequencing.

\section{ACT 1.10.R}

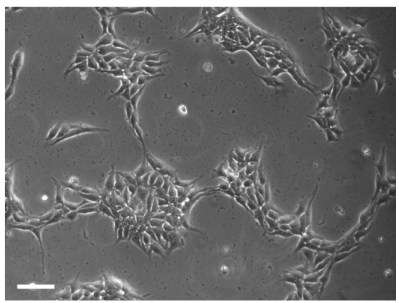

non-transfection control

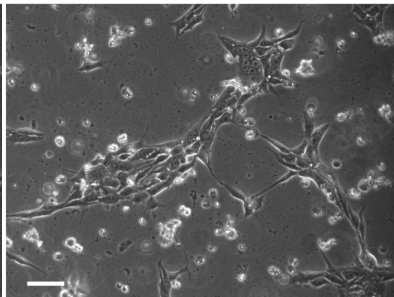

No HDR enhancer

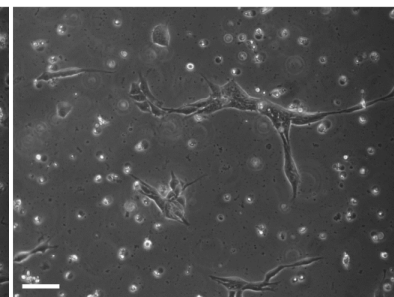

$20 \mu \mathrm{M}$ HDR enhancer

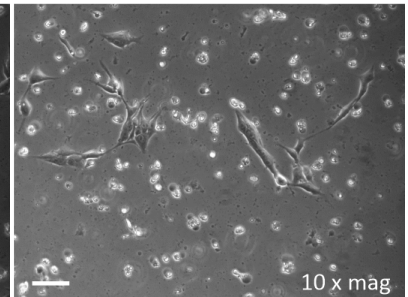

$30 \mu \mathrm{M}$ HDR enhancer

Figure 5-10: Representative bright field images of iPSCs one day after electroporation for CRISPR/Cas9 gene editing.

To enhance efficiency of the HDR, a HDR enhancer solution was added to the culture medium at concentrations of $20 \mu \mathrm{M}$ and $30 \mu \mathrm{M}$. Non-transfected iPSCs showed no signs of distress (far left), while transfected iPSCs detached from the plate (center left). HDR enhancer-treated iPSCs showed lower cell density and visibly more debris in the culture medium (center and far right). 10x magnification, scale bars $=20 \mu \mathrm{m}$.

\subsubsection{Amplicon sequencing}

Sanger sequencing is not suitable to detect SNPs in mixed samples, because the low number of reads does not allow a distinction between reading errors and the actual nucleotide polymorphism. Sequencing with a higher number of reads, so-called ultra-deep sequencing of PCR products or amplicon sequencing, allows confident detection of differences in specific regions of the genome. Therefore, the percentage of positive gene-

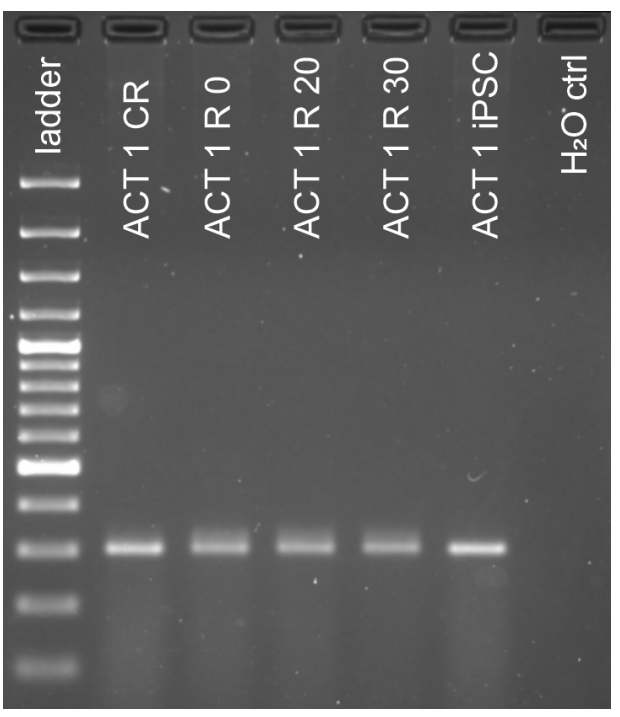

Figure 5-11: PCR product of RAC2 amplification

Electroporated cells (ACT 1 CR, ACT 1R) and untreated controls (ACT 1 iPSC) were tested for RAC2 mRNA expression. PCR products for RAC2 showed bands at the expected height of $299 \mathrm{bp}$. 
edited cell clones can be predicted and the number of clones calculated for analysis.

Six days after electroporation, the electroporated cells were detached, pooled, and counted (Figure 5-9). 6600 cells were used for singularization. The rest were divided into two halves: one portion was frozen for future culturing and the other part was harvested to test if the HDR emplate was introduced. To this end, genomic DNA was isolated and forward and reverse primers for RAC2 were used to amplify the region of interest with PCR. Five $\mu \mathrm{l}$ of the PCR product were loaded and run on an $1.5 \%$ agarose gel to confirm the correct size of the product and specificity of the primers (Figure 5-11). Bands for Rac2 ran at the expected height of $299 \mathrm{bp}$ and no nonspecific bands were visible, indicating high primer specificity (Figure 5-11). Subsequently, $500 \mathrm{ng}$ of PCR product were used for amplicon sequencing, which was carried out by GENEWIZ (South Plainfield, NJ, USA).

Table 5-2: Amplicon sequencing results from batches of iPSCs after CRISPR/Cas9 editing.

\begin{tabular}{|c|c|c|c|c|c|c|}
\hline Batch & SNP & $\begin{array}{l}\text { Total } \\
\text { Reads }\end{array}$ & Insertions & Deletions & $\begin{array}{l}\text { Indel } \\
\text { frequency }\end{array}$ & $\begin{array}{c}\text { HDR } \\
\text { frequency } \\
\text { (homozygous) }\end{array}$ \\
\hline $\begin{array}{c}\text { ACT1.10_R } \\
\text { no HDR } \\
\text { enhancer }\end{array}$ & \multirow{3}{*}{$\begin{array}{c}\text { rs13058338 } \\
\text { (RAC2) }\end{array}$} & 57050 & 3875 & 6817 & $\begin{array}{c}10692 \\
(44.6 \%)\end{array}$ & $\begin{array}{c}1573 \\
(6.6 \%)\end{array}$ \\
\hline $\begin{array}{c}\text { ACT1.10_R } \\
20 \mu \mathrm{M} \text { HDR } \\
\text { enhancer }\end{array}$ & & 56321 & 3105 & 6370 & $\begin{array}{c}9475 \\
(39.1 \%)\end{array}$ & $\begin{array}{c}2196 \\
(9.1 \%)\end{array}$ \\
\hline $\begin{array}{c}\text { ACT1.10_R } \\
30 \mu \mathrm{M} \text { HDR } \\
\text { enhancer }\end{array}$ & & 52624 & 2616 & 5616 & $\begin{array}{c}8232 \\
(36.3 \%)\end{array}$ & $\begin{array}{c}2268 \\
(10.0 \%)\end{array}$ \\
\hline
\end{tabular}

The results of the amplicon sequencing indicated the percentage of alleles that contain the desired HDR template (Table 5-2). Furthermore, Table 5-2 contains the total sequencing reads per sample (total reads), the number of detected insertions in the target sequence, and the number of deletions in the sequence. The sample without HDR enhancer had the greatest number of reads $(57,050)$, followed by the lower HDR enhancer concentration $(56,321)$ and the higher HDR enhancer concentration $(52,624)$. Most insertions were detected in the no-HDR-enhancer sample (3875), followed again by the samples with low (3105) and high (2616) HDR enhancer concentration. The trend continues when looking at deletions: Deletions were detected roughly twice as often as insertions, with the greatest number in the sample without enhancer (6817), followed by $20 \mu \mathrm{M}$ HDR enhancer (6370), and $30 \mu \mathrm{M}$ HDR enhancer. The indel frequency of the tested batches decreased from $44.6 \%$ 
(no enhancer) to $36.3 \%$ (30 $\mu \mathrm{M}$ HDR enhancer) with increasing HDR enhancer concentrations. More importantly, the HDR template frequency increased with HDR enhancer from $6.6 \%$ without enhancer to $10.0 \%$ with $30 \mu \mathrm{M}$ HDR enhancer. There was only a small difference in the occurrence of HDR frequencies between $20 \mu \mathrm{M}$ HDR enhancer $(9.1 \%)$ and $30 \mu \mathrm{M}$ HDR enhancer (10.0\%). These values gave a rough estimate of how many single iPSC colonies needed to be picked, cultured, harvested and analyzed in order to find the desired quantity of correctly edited clones.

\subsubsection{Sanger sequencing of individual clones}

While amplicon sequencing was carried out, 48 clones were picked from the ACT 1.10_R cell line. Each clone was transferred into one well of a 12-well plate and cultured until confluent enough for passage into one well of a 6 -well plate. Once the clones in the 6-well plate reached confluency, the cells were harvested, snap frozen in liquid nitrogen, and stored at $80^{\circ} \mathrm{C}$. The results from amplicon sequencing allowed us to estimate the success rate of gene editing. To precisely determine the actual number of homozygously and heterozygously edited clones, Sanger sequencing of individual clones was performed. Genomic DNA was isolated, Rac2 was amplified with specific primers, and PCR products were sent for Sanger sequencing (carried out by Microsynth AG, Balgach, Switzerland). 


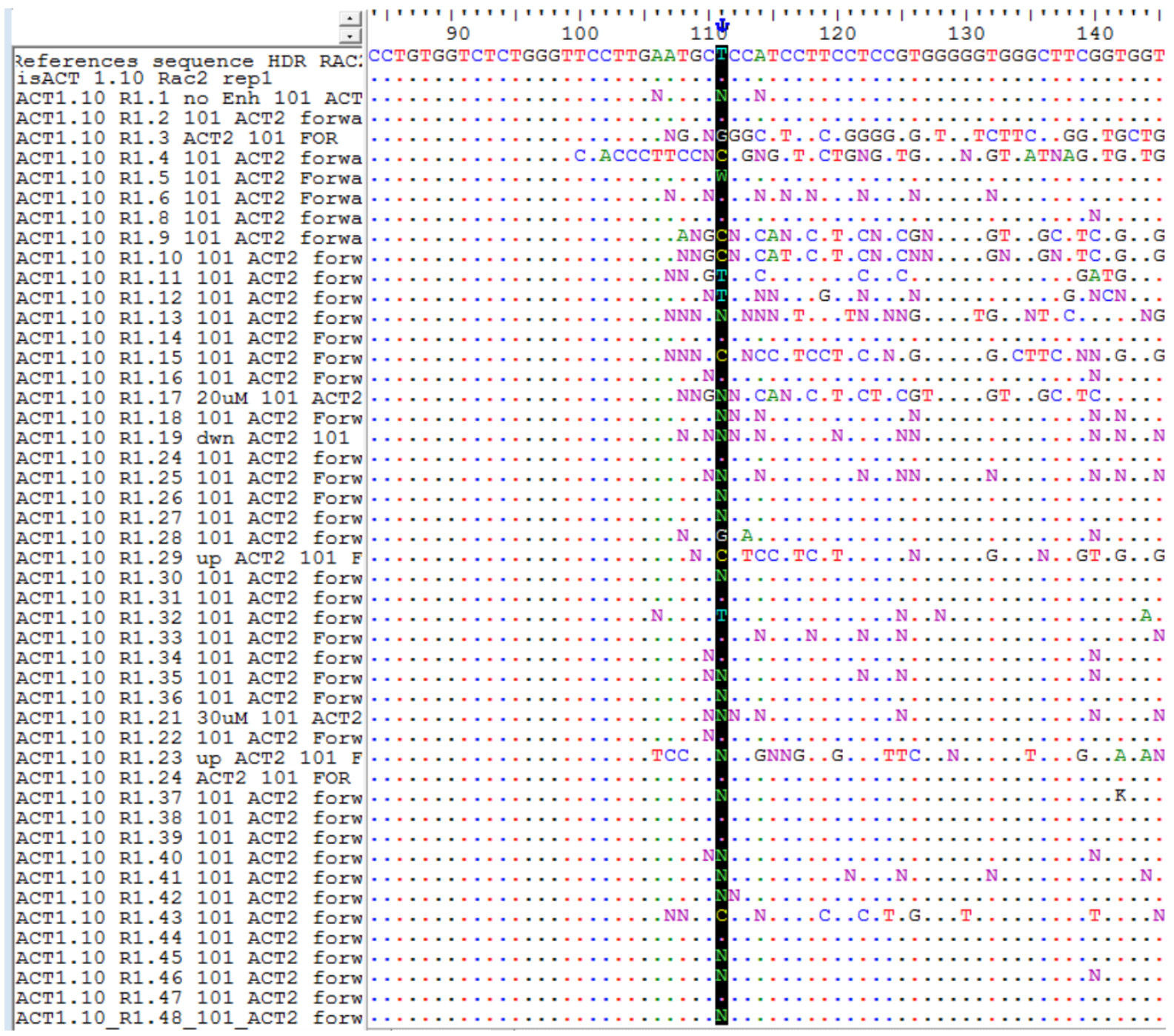

Figure 5-12: Screen shot from the BioEdit software.

Sequences from CRISPR/Cas9-edited clones were compared to a reference sequence (top). Matching nucleotides are indicated with a dot in the corresponding color. Homozyously aberrant nucleotides were named ( $C, T, G, A)$ and heterozygous or indefinite aberrations were marked as $\mathrm{N}$. The location of the SNP was highlighted in black.

Sanger sequencing results were lined up and visualized with the BioEdit software (Figure 5-12). The software automatically evaluated what kinds of edits occurred (Table 5-3). The results showed that regardless of HDR enhancer treatment, heterozygous edits were more common than homozygous edits. While heterozygous edits were much more frequent in iPSCs treated with HDR enhancer (56.25\%), homozygous edits seemed to be more frequent in untreated cells $(12.5 \%)$. 
Table 5-3: Editing efficiency of approaches with and without addition of HDR enhancer according to BioEdit software.

Editing was tested with Sanger sequencing of the genomic region around the target site.

\begin{tabular}{c|c|c|c|c} 
Approach & $\begin{array}{c}\text { \# clones } \\
\text { sequenced }\end{array}$ & $\begin{array}{c}\text { \# clones with } \\
\text { edits (\%) }\end{array}$ & $\begin{array}{c}\text { homozygous } \\
\text { edits (TT) }\end{array}$ & $\begin{array}{c}\text { heterozygous } \\
\text { edits (AT) }\end{array}$ \\
\hline $\begin{array}{c}\text { ACT1.10_R } \\
\text { no HDR enhancer }\end{array}$ & 16 & $\begin{array}{c}6 \\
(37.5 \%)\end{array}$ & $\begin{array}{c}2 \\
(12.5 \%)\end{array}$ & $\begin{array}{c}4 \\
(25 \%)\end{array}$ \\
\hline $\begin{array}{c}\text { ACT1.10_R } \\
\text { 20 } \mu \text { M HDR enhancer }\end{array}$ & 16 & $\begin{array}{c}10 \\
(62.5 \%)\end{array}$ & $\begin{array}{c}(6.25 \%) \\
(56.25 \%)\end{array}$ \\
\hline $\begin{array}{c}\text { ACT1.10_R } \\
\text { 30 } \mu \text { M HDR enhancer }\end{array}$ & 16 & 9 & 0 & 9 \\
& & $(56.25 \%)$ & $(0 \%)$ & $(56.25 \%)$
\end{tabular}

Upon closer assessment of the sequencing peaks, it became apparent that some sequencing results were inconclusive and needed more detailed investigation ('uninformative'). This could be due to a "messy" genotype after editing or there might be clones with a mixed allele type. Reviewing every sequencing plot individually painted a different picture than the initial results (Table 5-4). The number of edited clones per approach stayed the same. However, the rate of uninformative results was much higher in samples with HDR enhancer (37.5\% in both cases) compared to non-treated (6.25\%). CRISPR/Cas9 editing without subsequent HDR enhancer treatment resulted in one clone with a homozygous edit and four clones with heterozygous edits. The batch treated with $20 \mu \mathrm{M}$ HDR enhancer resulting also in one clone with a homozygous edit and only in three clones with heterozygous edits. Clones from the batch with $30 \mu \mathrm{M}$ HDR enhancer performed the worst, without any clones with homozygous edits and three clones with heterozygous edits.

Table 5-4: Sanger sequencing of individual clones from CRISPR/Cas9-edited iPSCs.

\begin{tabular}{c|c|c|c|c|c} 
Approach & $\begin{array}{c}\text { \# clones } \\
\text { sequenced }\end{array}$ & $\begin{array}{c}\text { \# clones with } \\
\text { edits (\%) }\end{array}$ & $\begin{array}{c}\text { homozygous } \\
\text { edits (TT) }\end{array}$ & $\begin{array}{c}\text { heterozygous } \\
\text { edits (AT) }\end{array}$ & Uninformative \\
\hline $\begin{array}{c}\text { ACT1.10_R } \\
\text { no HDR enhancer }\end{array}$ & 16 & $\begin{array}{c}6 \\
(37.5 \%)\end{array}$ & $\begin{array}{c}1 \\
(6.25 \%)\end{array}$ & $\begin{array}{c}(25 \%) \\
(6.25 \%)\end{array}$ \\
\hline $\begin{array}{c}\text { ACT1.10_R } \\
20 \mu \text { M HDR enhancer }\end{array}$ & 16 & $\begin{array}{c}10 \\
(62.5 \%)\end{array}$ & $(6.25 \%)$ & $(18.75 \%)$ & 6 \\
\hline ACT1.10_R & 16 & 9 & 0 & 3 & $(37.5 \%)$ \\
$30 \mu$ M HDR enhancer & & $(56.25 \%)$ & $(0 \%)$ & $(18.75 \%)$ & $(37.5 \%)$
\end{tabular}

Successfully edited iPSC lines are currently cultured and tested for stable gene editing with Sanger sequencing (Figure 5-13). To confirm heterozygous and homozygous iPSC lines, singularization steps will follow. A conclusion about the use of the HDR enhancer can only be drawn after the complete assessment of the gene-edited iPSC lines. 
ACT 1 R1.26

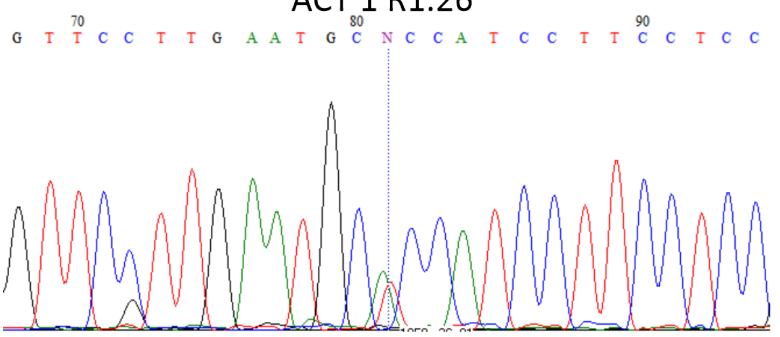

AT
ACT 1 R1.32

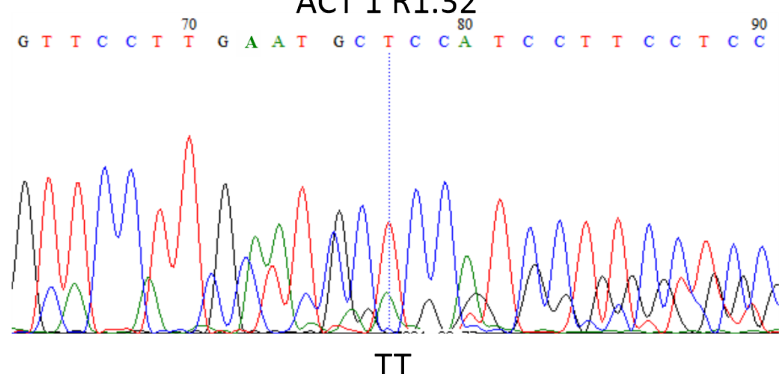

Figure 5-13: Representative depiction of Sanger sequencing results.

The sequence on the left was the iPSC line ACT 1 R1.26 that was edited at the site of the SNP rs13058338. The edit resulted in the genotype AT at the site of the SNP (left). In ACT 1 R1.32, the edit resulted in a homozygous edit (TT) at the relevant location (right).

\subsection{Telomere length and elongation}

Telomeres are repetitive DNA sequences at the end of chromosomes that cap and shield the chromosomes from damage and fusion (Blasco, 2005). Telomeres shorten with every cell division and decrease over our lifetimes because the telomerase enzyme complex, which can elongate telomeres, is silenced in the majority of cell types at birth (Blasco, 2005). Activation of TERT, the catalytic subunit of the telomerase enzyme complex, in adults supports cancer cell survival and drives cancer progression (Liu, 1999; Ducrest et al., 2002). Hence, most telomere research has been related to cancer or aging (Hanahan and Weinberg, 2000; López-Otín et al., 2013). With the finding that telomerase activity is linked to mtDNA protection (Haendeler et al., 2009), and that TERT overexpression reduces endogenous ROS production (Indran et al., 2011), telomerase research became highly relevant for the field of anthracycline-induced cardiotoxicity. Furthermore, it was reported that telomerase activation increased cellular lifespan by restoring telomeres that were shortened by oxidative stress (von Zglinicki, 2002, 2006). More recently, it was shown that cardiomyocyte-specific telomere shortening plays a role in age-associated and genetic forms of DCM (Chang et al., 2016; Chang and Blau, 2018). Furthermore, telomerase activation has protective effects in mice after MI (Bär et al., 2014). In addition, telomerase has antiapoptotic characteristics in CM (Oh and Schneider, 2002). Telomerase activity is not only interesting when looking at $\mathrm{CM}$. In stem cells, telomere stability is maintained through balancing elongation and trimming (Flores et al., 2005; Sharpless and DePinho, 2007).

\subsubsection{Telomere length}

Our collaborators, Prof. Thum and Dr. Bähr at the Medizinische Hochschule Hannover, previously detected telomere shortening in mice after DOX treatment (Bär et al., 2014; Bär and Thum, 2017). With regard to ACT modeling in iPSC-CMs, we wanted to address two questions:

1) Does telomere length differ between iPSCs and corresponding iPSC-CMs? 
2) Does telomere length differ between iPSC-CMs from control patients compared to iPSC-CMs from ACT patients?

To determine telomere length in IPSCs and iPSC-CMs, we prepared samples for PCR and qFISH, and sent them to Hanover for analysis, where PhD candidate Shambhabi Chatterjee carried out the assay in the lab of Dr. Christian Bähr.
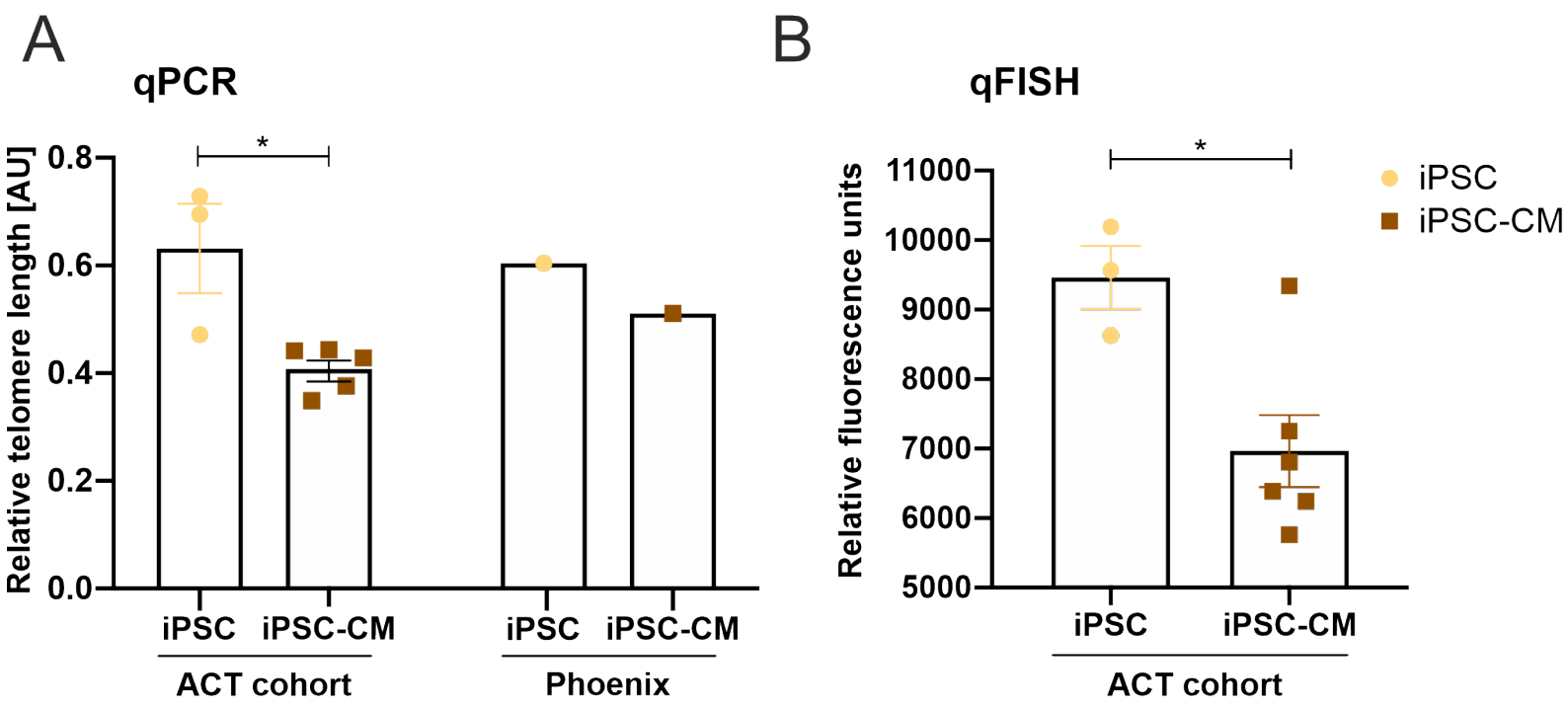

Figure 5-14: Telomere lengths differed between iPSCs and iPSC-CMs.

Telomere lengths were measured with qPCR and qFISH. In addition to iPSCs and iPSC-CMs from ACT patients and controls, Phoenix iPSCs and iPSC-CMs were included as an internal control. A: qPCR results indicate that telomeres of iPSCs are longer than telomeres of matched iPSC-CMs. B: $\mathrm{qFISH}$ results show that telomeres are longer in iPSCs than in iPSC-CMs.. AU=Arbitrary unit. A: iPSC: $n=$ three samples from three cell lines from two patients. iPSC-CMs: $n=$ six independent differentiations from three cell lines, from two patients. (Phoenix: $n=1$ ) B: iPSC: $n=$ three samples from three cell lines from two patients. iPSC-CMs: $\mathrm{n}=$ five independent differentiations from three cell lines, from two patients. Phoenix was used as a positive control. Statistical analysis was performed with an unpaired t-test. $\mathrm{P}$ values were represented as followed: $\mathrm{p}>0.05=* ; \mathrm{p}>0.01=* *$. Horizontal bars indicate the mean $+/$ - SEM.

To answer the question whether telomere length changes during cardiac differentiation, we analyzed telomere lengths in iPSCs and iPSC-CMs from ACT patient and control cell lines via quantitative PCR (qPCR) and qFISH. The analysis of telomeric repeats via qPCR showed that the relative length of telomeres is $50 \%$ longer in iPSCs than in iPSC-CMs (Figure 5-14A). An additional way to determine telomere length is with $\mathrm{qFISH}$. With this approach iPSC telomeres were 1.3-fold longer than iPSC-CM telomeres (Figure 5-14B). Hence, the qFISH results confirmed that telomeres of iPSCs are longer than telomeres of iPSC-CMs. 


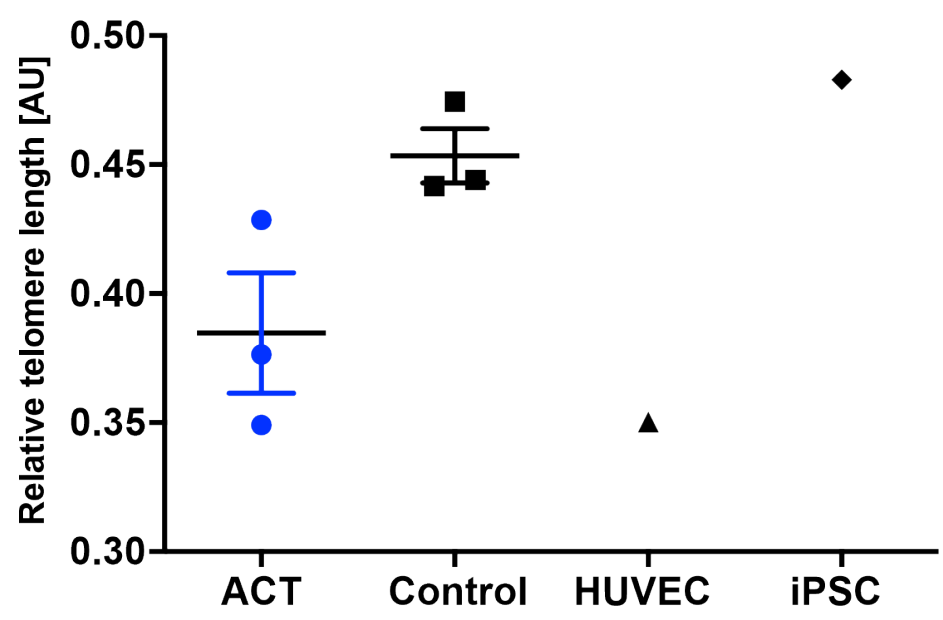

Figure 5-15: qPCR results from telomere analysis.

Telomere analysis of iPSC-CMs indicated that telomeres were shorter in ACT patients compared to control patients. $n=$ three independent differentiation experiments of one cell line from one ACT and control patient, respectively. $\mathrm{AU}=$ arbitrary units. Statistical analysis was performed with an unpaired t-test. Horizontal bars indicate the mean +/- SEM. HUVEC cells were used as marker for short telomeres in comparison to Phoenix-iPSCs with longer telomeres.

In the next set of experiments, we compared explicitly the telomere length in iPSC-CMs from ACT patients with controls. We found that the relative length of telomeres was shorter in ACT-iPSC-CMs compared to control-iPSC-CMs (Figure 5-15).

This initial set of experiments showed that there are differences in telomere length between iPSCs and iPSC-CMs. Furthermore, iPSC-CMs from ACT patients were found to have shorter telomeres compared to control patients. Clearly, these preliminary data sets need to be supported by additional observations. So far, there is an indication that telomeres are shorter in iPSC-CMs from ACT patients compared to control patients.

\subsubsection{Optimization of AAV6-DOX-apoptosis assay}

Telomerase activity in adult cardiomyocytes is thought to be advantageous against senescence and aging. A decline in telomerase activity in adult rat hearts through aging has been described (Leri et al., 2000). TERT expression and telomerase activity are downregulated in the adult rat heart (Oh and Schneider, 2002). Longer telomeres are thought to render cells more resistant to DOX-related cytotoxicity (Bär et al., 2014; Beyer et al., 2016; Quryshi et al., 2018). Our approach to achieve longer telomeres was to transduce iPSC-CMs with AAV6 vectors overexpressing telomerase regulated by a CMV promoter. To determine the optimal MOI, iPSC-CMs were transduced with AAV6-green fluorescent protein (GFP) vectors, which allowed for a simple read-out of transduction rate. We tested MOls of $1 \times 10^{3}$, $5 \times 10^{3}$, and $1 \times 10^{4}$.

Transduced iPSC-CMs were monitored with a fluorescent microscope. The first green fluorescent iPSC-CMs were visible after $24 \mathrm{~h}$ in wells that were infected with MOls of $5 \times 10^{3}$ and $1 \times 10^{4}$, respectively (Figure 5-16A). After $48 \mathrm{~h}$, fluorescent iPSC-CMs were visible in all 
AAV6-GFP-infected wells. The highest number of fluorescent iPSC-CMs was detected six days after infection, with little difference between the MOls $5 \times 10^{3}$ and $1 \times 10^{4}$.

A

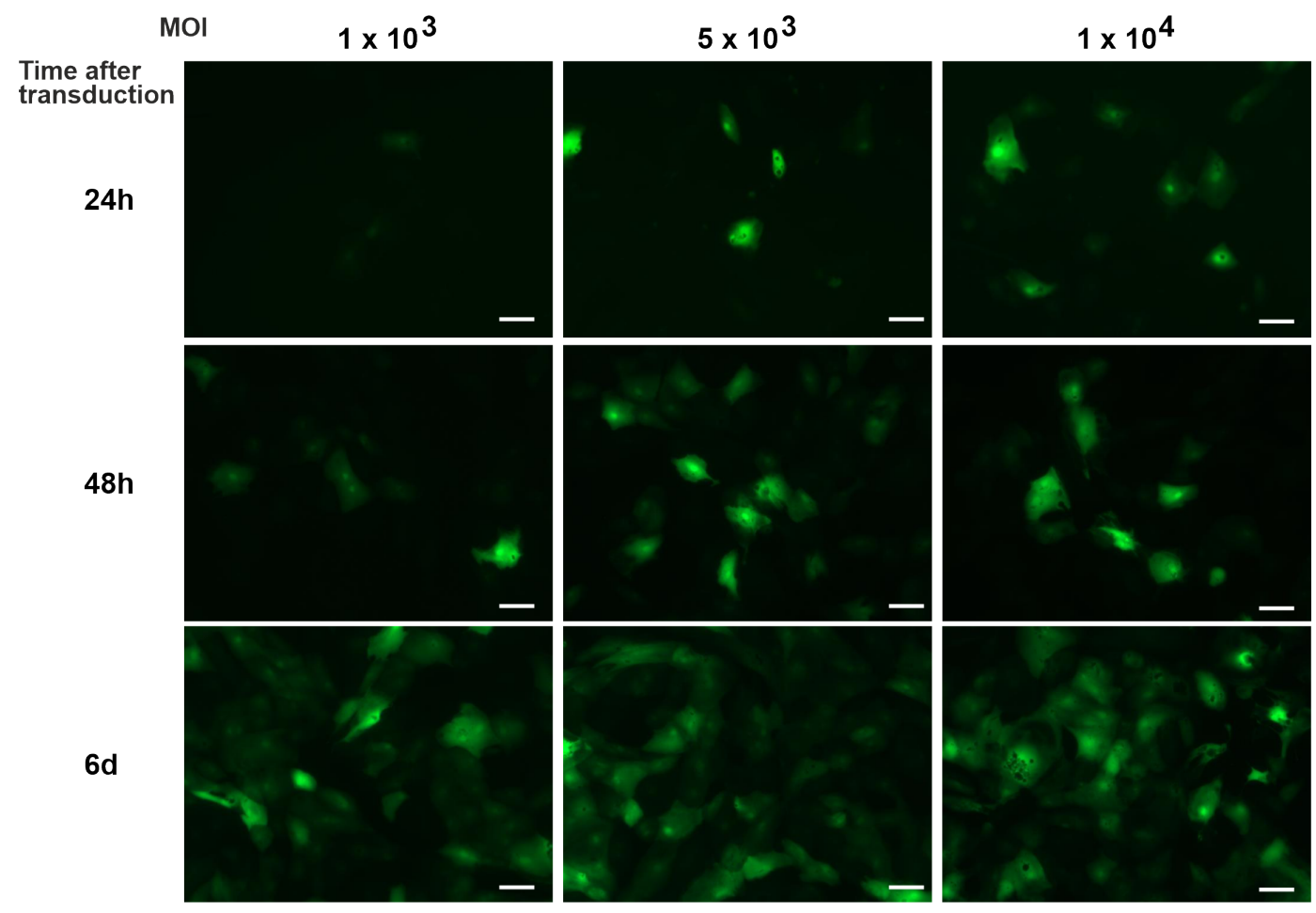

B
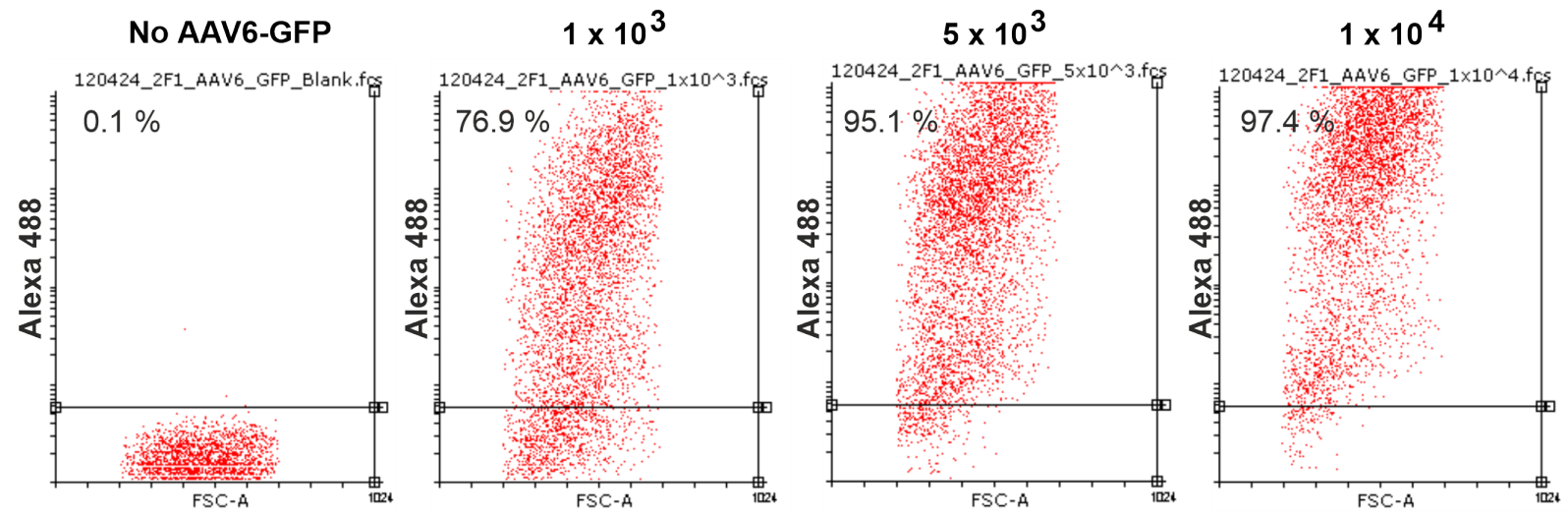

Figure 5-16: MOI optimization for AAV6 infection of iPSC-CMs.

iPSC-CMs from a control line were transduced with three different MOIs. A: Fluorescent images show treated cells at $24 \mathrm{~h}, 48 \mathrm{~h}$ and six days after transduction. First fluorescent iPSC-CMs were visible after $24 \mathrm{~h}$ in wells that were treated with MOls of $5 \times 10^{3}$ and $1 \times 10^{4}$. After $48 \mathrm{~h}$, fluorescent iPSCCMs were visible in all AAV6-GFP-infected wells. Scale bars $=20 \mu \mathrm{M}$. B: Representative scatter plots visualize the portion of GFP-positive cells six days after AAV6-GFP transduction measured with flow cytometry. For each plot 10,000 iPSC-CMs were measured. Untreated iPSC-CMs were used to determine the baseline (left). Increasing MOIs resulted in increased percentages of GFP-positive cells. The highest number of fluorescent cells was detected after six days, with $95.1 \%$ in iPSC-CMs transduced with an $\mathrm{MOI}$ of $5 \times 10^{3}$, and $97.4 \%$ in iPSC-CMs with an MOI of $1 \times 10^{4}$. 
A more detailed measure of GFP-positive iPSC-CMs was achieved with flow cytometry. On day six, iPSC-CMs were detached and analyzed for GFP expression. Using an MOI of $1 \mathrm{x}$ $10^{3}$, the transduction rate was $77 \%$. An MOI of $5 \times 10^{3}$ resulted in $95 \%$ GFP-positive cells six days after transduction (Figure 5-16B). Doubling the MOI to $1 \times 10^{4}$ increased the transduction rate only to $97 \%$ GFP-positive cells. Therefore, we determined $5 \times 10^{3}$ as our $\mathrm{MOI}$ of choice and used it for the following AAV6-tert experiments.

We hypothesized that iPSC-CMs with enhanced telomerase activity would be more resistant to DOX treatment than iPSC-CMs with low telomerase activity. Initial transductions showed that AAV6 infection alone caused cell stress, which manifested in detached iPSC-CMs and debris in the culture medium (Figure 5-17). These indicators for cellular stress were observed with bright field microscopy in iPSC-CMs that were infected either with AAV6 GFP or AAV6 TERT (Figure 5-17 center and right). Four days after transduction, iPSC-CMs that were infected with AAV6 TERT showed decreased cell density (Figure 5-17), indicating cell death. Hence, AAV6 transduction might increase apoptosis levels at baseline. To test for increased resistance to DOX treatment, we looked at apoptosis rates with the Annexin $\mathrm{V}$ apoptosis kit for flow cytometry. To avoid increasing stress through DOX treatment and AAV6 transduction, DOX treatment was initiated $48 \mathrm{~h}$ after AAV6 transduction. iPSC-CMs were treated with $0.25 \mu \mathrm{M}$ DOX or $1 \mu \mathrm{M}$ DOX for $72 \mathrm{~h}$, and the apoptosis assay was carried out on day five after AAV6 transduction (Figure 5-18A).

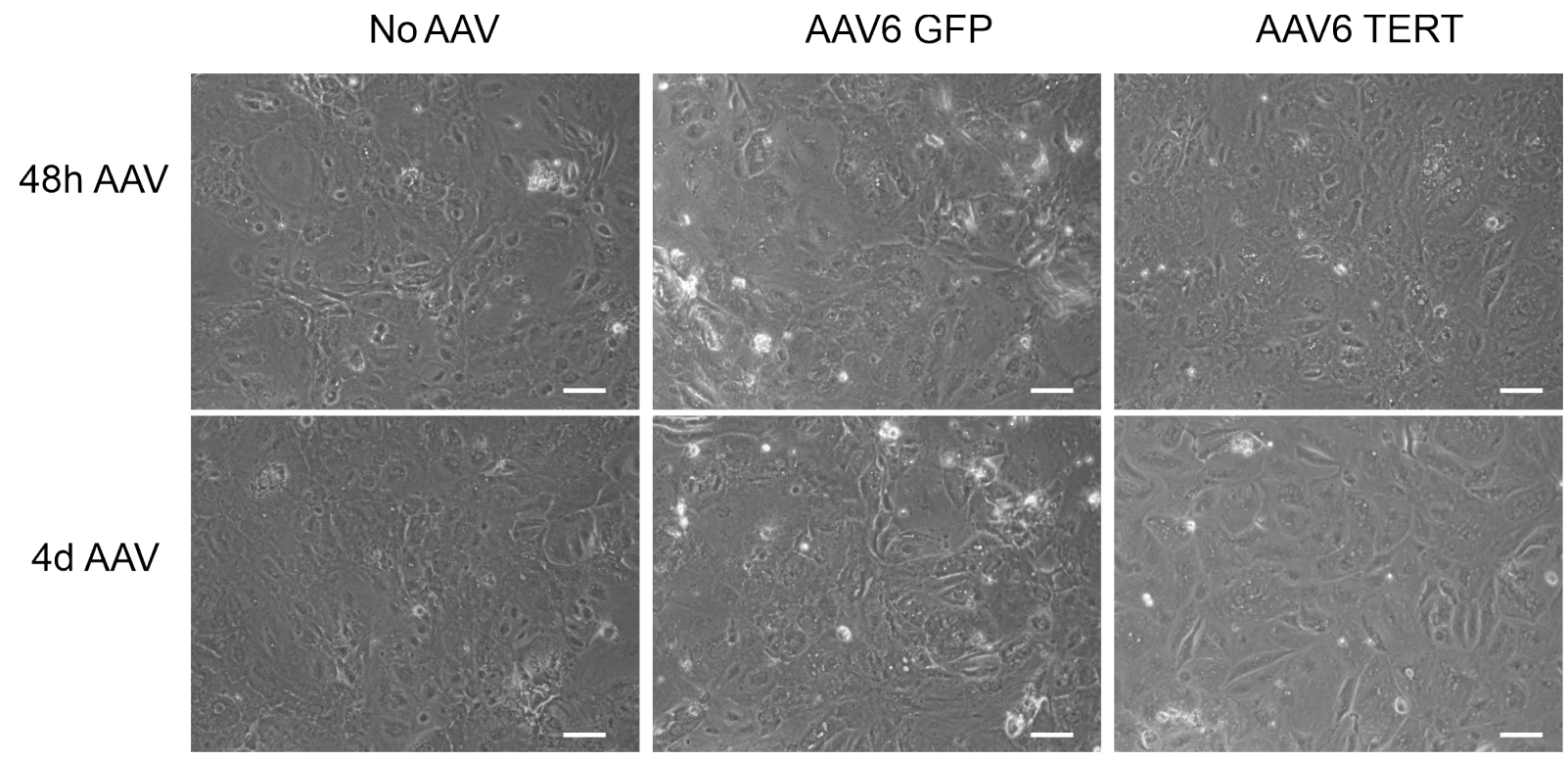

Figure 5-17: Bright field microscopy images of iPSC-CMs after AAV6 vector transduction.

AAV6-transduced iPSC-CMs were compared to uninfected controls. AAV6 transduction itself appeared to have a negative effect on iPSC-CMs, which was more prominent with AAV6-GFP transduction. Scale bars $=20 \mu \mathrm{m}$. 
Bright field microscopy images of iPSC-CMs, which were transduced with AAV6 for $48 \mathrm{~h}$ followed by DOX treatment for $72 \mathrm{~h}$ showed detached iPSC-CMs and a decrease in growth density (Figure 5-18B).

A

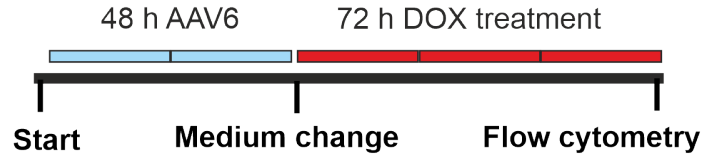

B

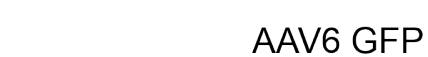

Doxorubicin

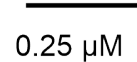

$1 \mu \mathrm{M}$

(AAV)

Oh

(48h)
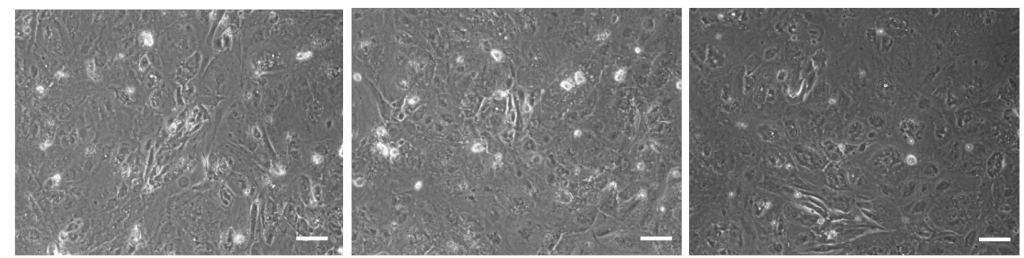

AAV6 TERT

$48 \mathrm{~h}$

$(4 d)$
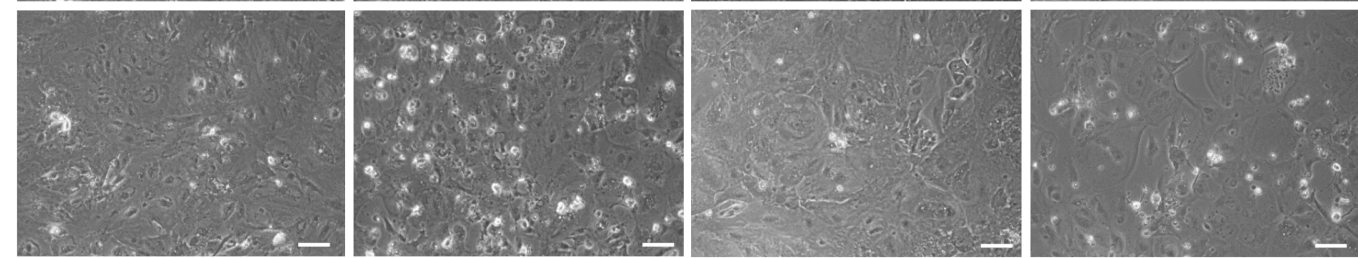

C
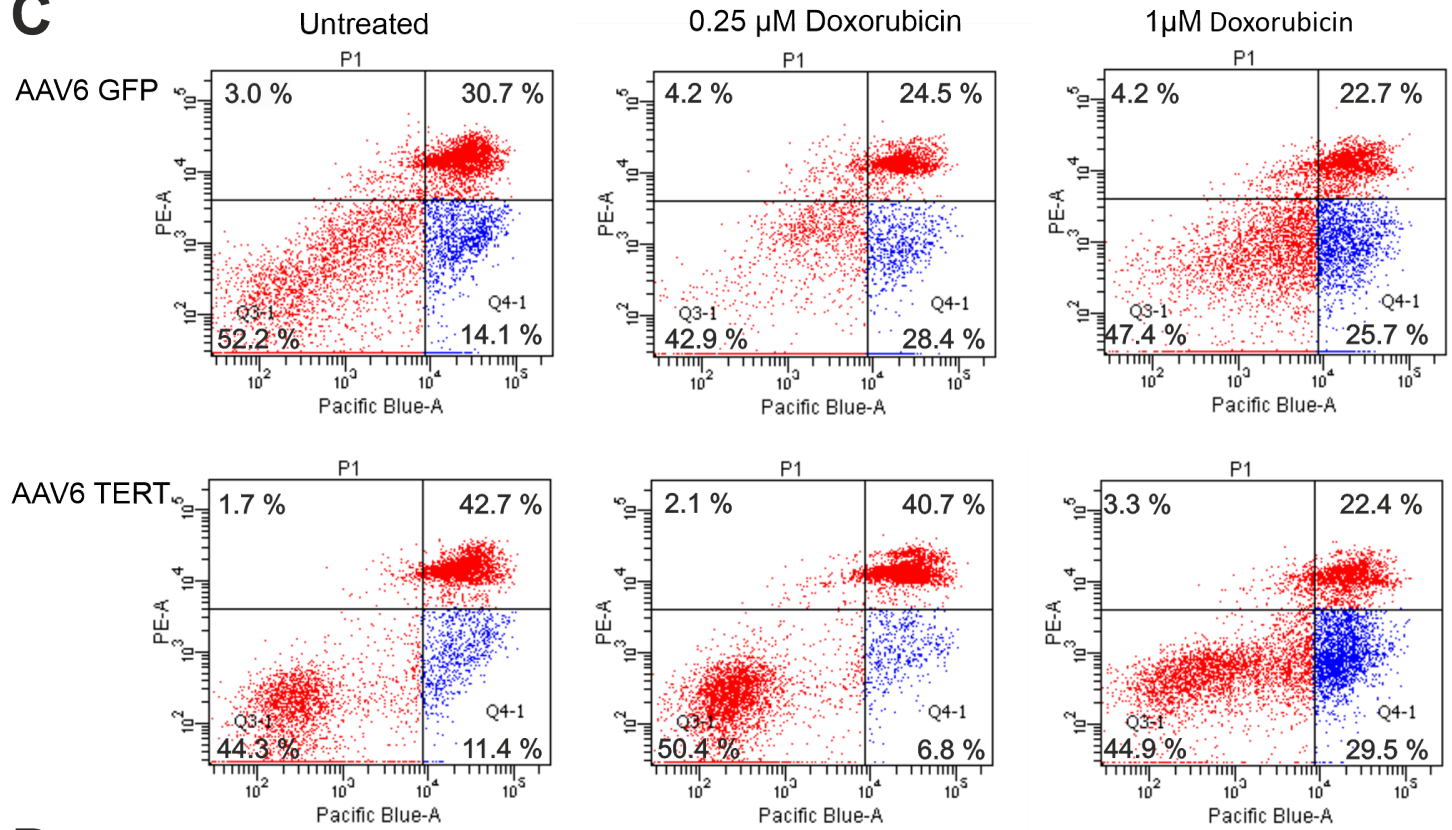

D

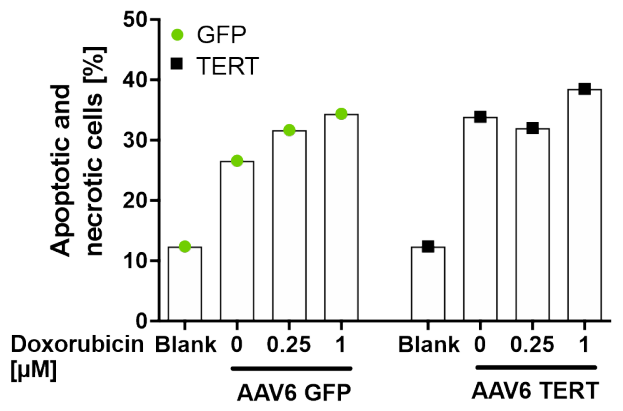

Figure 5-18: Apoptosis assay of DOX-treated, AAV6-transduced iPSC-CMs. 
A: Time line of AAV6 transduction and DOX treatment. B: Bright field microscopical images of iPSCCMs. iPSC-CMs were infected with AAV6 vectors and treated with $0.25 \mu \mathrm{M}$ DOX and $1 \mu \mathrm{M}$ DOX, respectively. AAV6-transduced- and DOX-treated iPSC-CMs were more often detached from the culture plate than untreated iPSC-CMs. Scale bars $=20 \mu \mathrm{M}$. C: Representative scatter plots of flow cytometry with Annexin V/PI apoptosis kit showed a shift in cell distribution after DOX treatment. D: Summary of flow cytometrical results. AAV6 vector transduction increased the percentage of apoptotic and necrotic iPSC-CMs. Treatment with DOX increased the apoptosis rate further. $\mathrm{n}=$ one differentiation of the ACT 3.11 iPSC line.

AAV6 transduction alone, regardless of AAV6 GFP or AAV6 TERT, was sufficient to result in detached iPSC-CMs (Figure 5-18B, top row). Additional DOX treatment increased the appearance of detached iPSC-CMs and $1 \mu \mathrm{M}$ DOX had a greater impact than $0.25 \mu \mathrm{M}$ DOX (Figure 5-18B). Apoptosis assays with flow cytometry confirmed the initial observation. Representative scatter blots show that AAV6 GFP-transduced iPSC-CMs were $48 \%$ apoptotic and necrotic (Figure 5-18C). With DOX treatment, this rate increased to $43 \%$ $(0.25 \mu \mathrm{M}$ DOX) and 47\% (1 $\mu \mathrm{M}$ DOX). iPSC-CMs that were transduced with AAV6 TERT had similar apoptosis and necrotic rates with 56\% (no DOX), 50\% (0.25 $\mu \mathrm{M}$ DOX), and 55\% $(1 \mu \mathrm{M}$ DOX). With both AAV6 vectors the apoptosis and necrosis rate was higher with $0.25 \mu \mathrm{M}$ DOX treatment compared to $1 \mu \mathrm{M}$ DOX treatment (Figure 5-18C). Due to the high stress in iPSC-CMs treated with $1 \mu \mathrm{M}$ DOX, iPSC-CMs might go into apoptosis or necrosis earlier, and cannot be detected by the flow cytometry assay at day five. The reduced iPSC$\mathrm{CM}$ density on the bright field images supported that explanation (Figure 5-18B). Compared to untreated iPSC-CMs, AAV6-transduced iPSC-CMs had a two to three times higher apoptosis rate (Figure 5-18D). Contrary to our expectations, DOX increased the apoptosis and necrosis rates less than AAV6 transduction. $1 \mu \mathrm{M}$ DOX treatment accounted for an increase of apoptosis and necrosis of eight (GFP) and four percentage points, respectively (Figure 5-18D).

To limit the stress on the iPSC-CMs and to allow for a longer recovery time, the treatment protocol was adjusted and DOX treatment was started seven days after the end of the AAV6 transduction (Figure 5-19A). Furthermore, DOX concentration was set to $0.5 \mu \mathrm{M}$, which was more tolerable to the iPSC-CMs than $1 \mu \mathrm{M}$ DOX. These adjustments should not negatively influence TERT expression, because it is stable for up to 14 days after transduction (Dr. Christian Bär, personal communication).

\subsubsection{AAV6 TERT apoptosis assay}

With the previously mentioned improvements, the flow cytometry apoptosis assay was repeated. Representative scatter plots showed that iPSC-CMs without DOX treatment had relatively low apoptosis and necrosis rates (Figure 5-19B). For AAV6-untransduced iPSCCMs and AAV6 GFP-transduced iPSC-CMs, the rate of non-apoptotic iPSC-CMs was approximately $75 \%$. AAV6 TERT-treated iPSC-CMs had a slightly lower non-apoptotic rate with $69 \%$ (Figure 5-19B). While apoptosis rates of DOX-untreated iPSC-CMs were nearly 
identical between empty AAV6, and AAV6-untransduced groups (11\%-15\%), apoptosis rates were slightly elevated with AAV6-TERT transduction alone (18\%; Figure 5-19C). Treatment with $0.5 \mu \mathrm{M}$ DOX led to an increase in apoptosis rates in all AAV6 conditions (Figure 5-19C). In AAV6-untransduced iPSC-CMs the DOX-induced increase of apoptosis rate was 2.8- and 1.8-fold in ACT- and control iPSC-CMs, respectively (Figure 5-19D). In empty AAV6transduced iPSC-CMs, the increase after DOX treatment was the lowest with 1.8-fold in ACT-iPSC-CMs and 1.5-fold in control iPSC-CMs. TERT-transduced iPSC-CMs showed an increase of DOX-induced apoptosis of 2-fold and 1.5-fold for ACT and control iPSC-CMs, respectively (Figure 5-19D).

Overall, AAV6-TERT transduction did not lead to a significant decrease of apoptosis and necrosis rates. Notably, there was a non-significant trend that DOX-induced apoptosis and necrosis rates increased more in ACT-iPSC-CMs compared to control-iPSC-CMs (Figure 519C, D), which is in line with previous findings of our group (Haupt, 2018). Apoptosis and necrosis rates decreased slightly with empty AAV6 transduction compared to AAV6untransduced iPSC-CMs after DOX treatment, but no vector rescued the iPSC-CMs from DOX-induced apoptosis and necrosis. 
A

\begin{tabular}{|l|l|l|l|}
\hline Start & Medium change & Medium change & Medium change
\end{tabular}

B
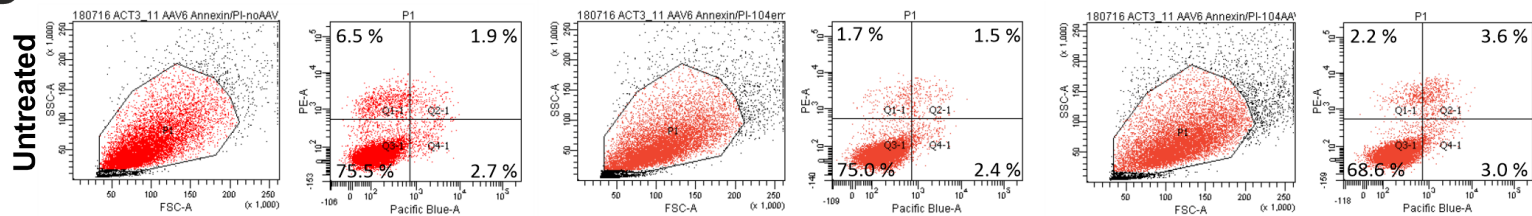

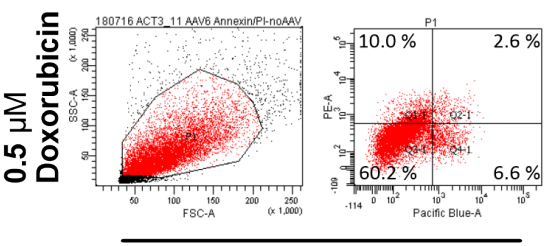

Blank

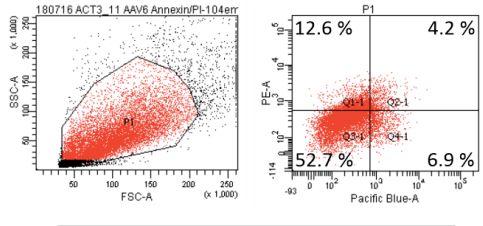

Empty AAV6

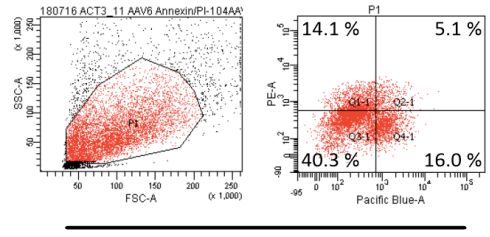

AAV6 TERT

C

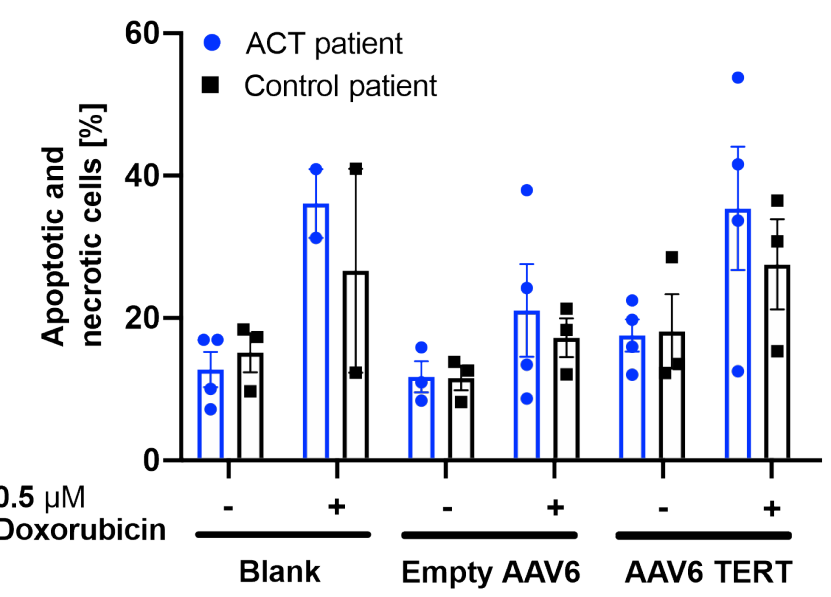

D

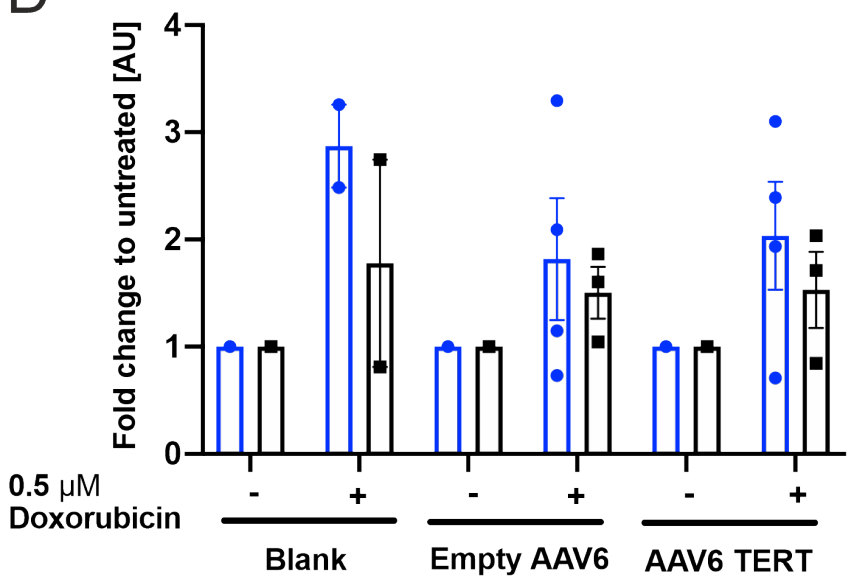

Figure 5-19: Flow cytometry apoptosis assay of iPSC-CMs after TERT overexpression and DOX treatment.

A: Time line of AAV6 transduction, recovery period, and DOX treatment. B: Representative scatter plots show gate setting for cell size, and in the other field for red fluorescence (y axis) and blue fluorescence (x axis). Scatter plots show distinctly different cell distribution depending on DOX concentration. Panels of individual scatter plots indicate necrotic (Q1- top left), late apoptotic (Q2- 
top right), early apoptotic (Q3- bottom right), and non-apoptotic iPSC-CMs (Q4- bottom left). Numbers indicate the percent values of total cell count. The difference between the sum of percentage values and $100 \%$ is the number of counted events outside the gated population. C: Quantified flow cytometry data show that DOX treatment increased the mean percentage of apoptotic and necrotic iPSC-CMs in each group. D: Fold-change data indicates a significant increase in apoptotic iPSC-CMs after DOX treatment in the ACT patient group without vector treatment. The increase of DOX-induced apoptosis rate did not change significantly with vector treatment. $A U=$ arbitrary unit. $\mathrm{n}=$ one independent iPSC-CM differentiation. ACT patient and control patient: $n=$ two to four differentiations from one iPSC line from one patient, respectively. Statistical analysis was performed with two-way ANOVA. Horizontal bars indicate the mean +/- SEM.

\subsubsection{AAV6 TERT ROS assay}

Apoptosis can be triggered through different pathways and cells have a plethora of compensational mechanisms in place, so not every form of distress leads to self-destruction. Therefore, the measurement of the apoptosis rate has limitations when assessing the effects of DOX treatment. A more direct effect of DOX is the increase in ROS, which can be measured with the Amplex red assay (Zhou et al., 1997). iPSC-CMs were transduced with AAV6 vectors and challenged with $0.5 \mu \mathrm{M}$ DOX for $72 \mathrm{~h}$, as described before. Subsequently, the iPSC-CMs were incubated with an Amplex red probe and luminescence was measured with a plate reader. 

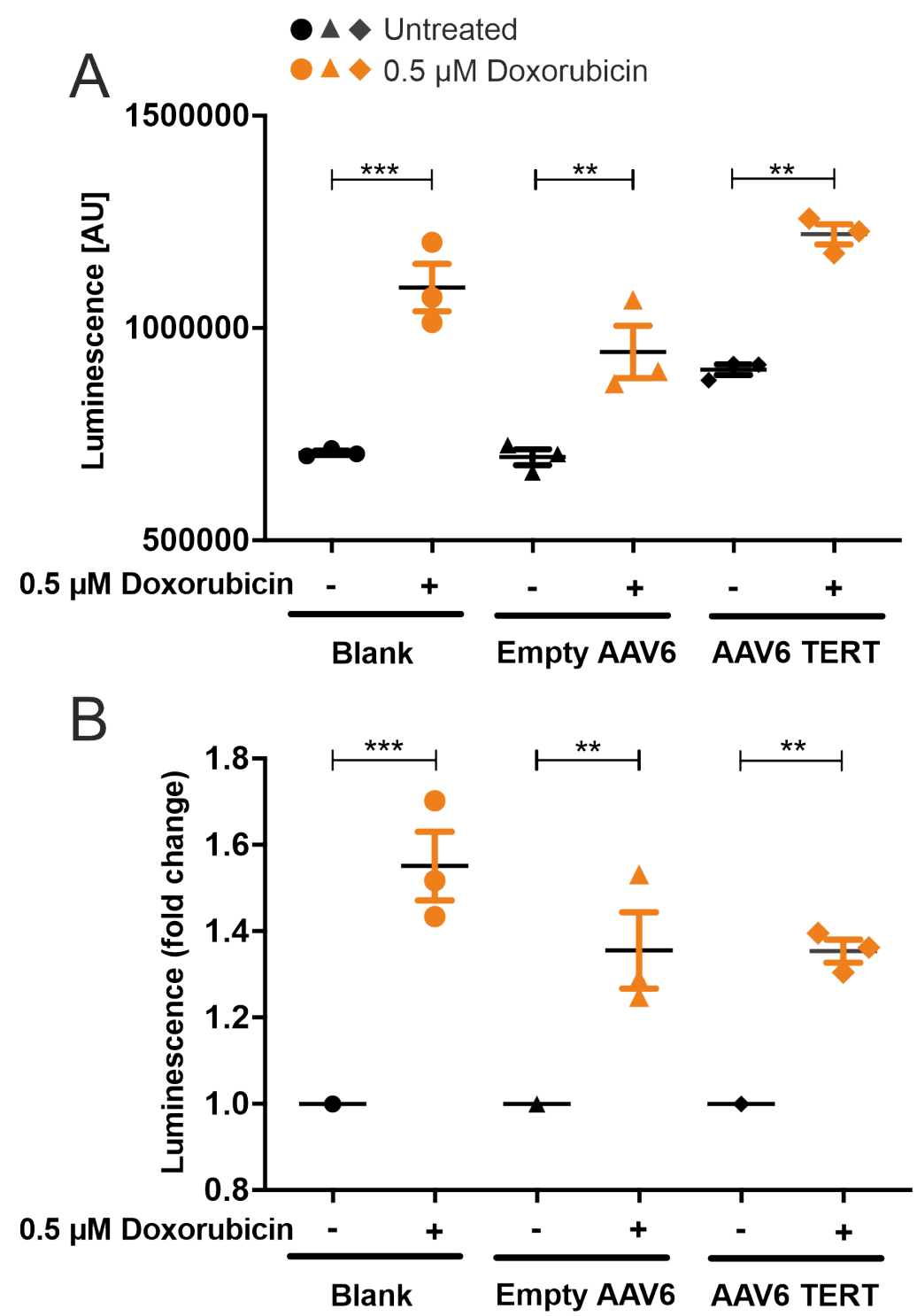

Figure 5-20: DOX treatment increased extracellular ROS in iPSC-CMs as measured with Amplex red.

ROS production of iPSC-CMs was indicated through Amplex red luminescence. iPSC-CMs from ACT patient 3 were infected with AAV6 vectors and treated with $0.5 \mu \mathrm{M}$ DOX. Uninfected iPSC-CMs served as reference. A: DOX-treated iPSC-CMs produced more ROS than untreated cells. This was consistent throughout the groups. B: When luminescence values were normalized to respective DOXuntreated controls, vector-treated samples had a smaller increase in ROS after DOX compared to blank controls. $\mathrm{AU}=$ arbitrary unit. $\mathrm{n}=$ single measurement of luminescence in one well of a 96-well plate. The dots per condition represent triplicate measurements of one differentiation from one iPSC line from ACT patient 3. P values were represented as followed: $p>0.05=* ; p>0.01{ }^{* *} ; p>0.005=$ $* * *$. Statistical analysis was performed with two-way ANOVA. Multiple comparisons were corrected according to Sidak. Horizontal bars indicate the mean +/- SEM.

In all groups, there was a significant increase in ROS after DOX treatment. AAV6 TERTtransduced iPSC-CMs produced more ROS at baseline compared to untreated cells and empty vector-treated iPSC-CMs (Figure 5-20A). The luminescence values after DOX treatment did not differ significantly between the groups. When normalized to respective untreated values, the fold-change increase in ROS after DOX treatment was slightly lower in vector- transduced samples, however, no difference was seen between empty AAV6 and 
AAV6 TERT (Figure 5-20B). Overall, the results of the Amplex red assay were comparable to the outcome of the apoptosis assay.

\subsection{Characterization of the DCM cohort}

ACT can be described as an induced form of DCM. To assess if some of the investigated disease mechanisms are specific to ACT, or if they are general heart failure symptoms, we added iPSC-CMs from DCM patients to the project.

One patient diagnosed with DCM end stage heart failure followed by heart transplantation and one healthy control donor were willing to donate somatic material for iPSC generation. We established and characterized iPSCs and iPSC-CMs from these individuals and included them in this study as a reference for heart failure without anthracycline induction. This helped to differentiate between ACT-specific disease mechanisms and general heart failure processes.

\subsection{1. iPSC characterization}

Reprogramming for all somatic cells of the different donors was performed in former studies of the group with the non-integrative Sendai virus (Table 3-1). Characterization of newly generated cells is crucial because iPSCs that have undergone only partial reprogramming might not be capable of differentiation into iPSC-CMs sufficiently (Koyanagi-Aoi et al., 2013; reviewed by Takahashi and Yamanaka, 2016). To confirm stemness and pluripotency, two cell lines per donor were selected and tested for stem cell-like morphology and expression of characteristic genes and proteins. 


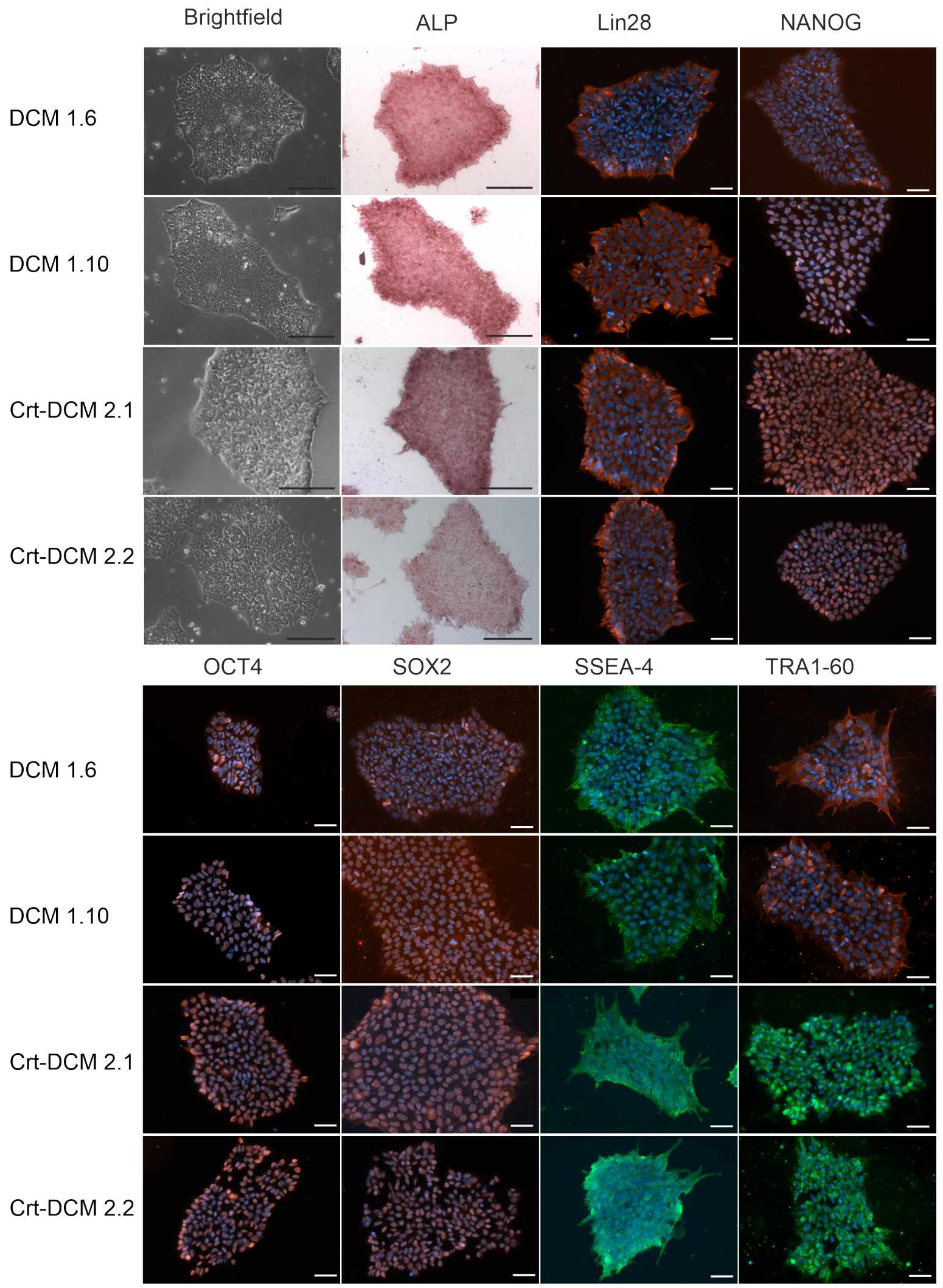

Figure 5-21: The generated iPSCs show pluripotency characteristics.

The iPSC lines (representative for DCM patient 1 (DCM 1.6 and DCM 1.10), and control (Crt-DCM 2.1 and Crt-DCM 2.2) showed typical morphology for human pluripotent stem cells and positive staining for alkaline phosphatase (top left panels). Immunocytochemistry staining with antibodies specific for LIN28, NANOG, Oct-4, SOX2, SSEA-4, and TRA-1-60, respectively, showed specific signals for respective targets. Scale bars $=50 \mu \mathrm{m}$. 


\subsubsection{Morphology, alkaline phosphatase expression, and pluripotency markers}

Stem cells have a characteristic morphology, as they are relatively small, round, and form colonies that have a clear boundary towards the rest of the plate. Investigated iPSCs from the DCM patient and the Crt-DCM donor showed typical morphology as described for human pluripotent stem cells and showed high alkaline phosphatase activity (Figure 5-21, top left panels). Presence of pluripotency proteins was investigated with immunofluorescence analysis. The acquired images revealed that the investigated cells were positive for pluripotency markers. Staining of transcription factors like homeobox protein NANOG (NANOG), Oct-4, and SOX2 resulted in nuclear signals. Immunopositive signals from the keratin sulfate antigen TRA-1-60 and stage-specific embryonic antigen 4 (SSEA-4) were seen in the cell membrane. The RNA-binding protein Lin-28 homolog A (LIN28; encoded by LIN28) (Tsialikas and Romer-Seibert, 2015) was located in the cytoplasm (Figure 5-21). To test the abundance of mRNA of pluripotency markers in the cells, we performed semiquantitative RT-PCR with primers for SOX2, Oct-4, NANOG, and LIN28. Equal loading was controlled through the housekeeping genes HPRT (for Crt-DCM 2.1 and 2.2) or GAPDH (for DCM 1.6 and DCM 1.10). All investigated pluripotency genes were up-regulated and highly expressed in the iPSC lines compared to the parental somatic cells (PBMCs from CrtDCM). Somatic cells showed a band for HPRT, but were negative for stem cell markers (Figure 5-22).

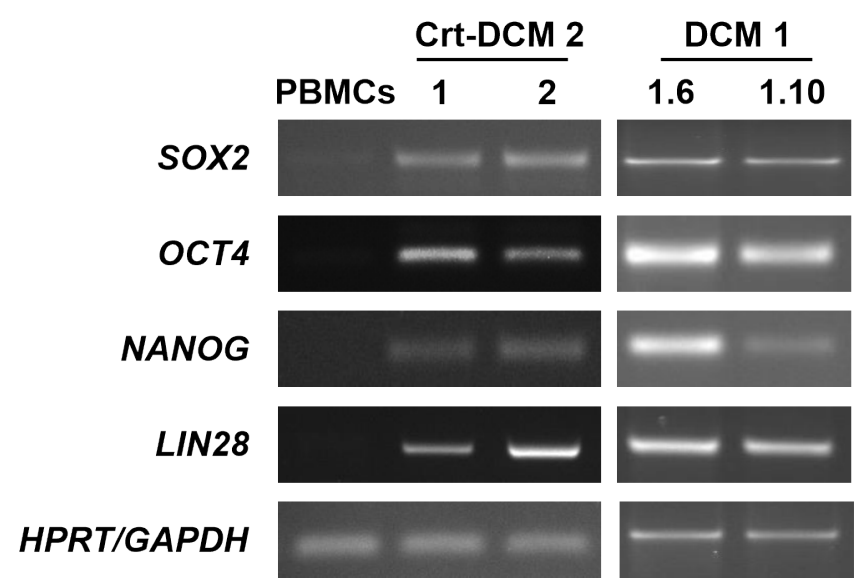

Figure 5-22: Expression of pluripotency genes in Crt-DCM and DCM lines on mRNA level.

Semiquantitative RT-PCR showed expression of SOX2, Oct-4, NANOG, and LIN28 in all analyzed iPSC lines. No expression was seen in PBMCs used for reprogramming of Crt-DCM 2.1 and Crt-DCM 2.2. HPRT was used as reference gene for Crt-DCM lines and GAPDH was used as reference gene for DCM 1-lines.

\subsubsection{Spontaneous differentiation potential in vitro}

The characterization of newly generated iPSCs included their capacity for spontaneous differentiation into three germ layers. For that purpose, we tested the potential for 
spontaneous differentiation in the iPSCs by using the EB methodology and the expression of specific markers for each germ layer. iPSCs of passages no higher than twelve were cultured together with MEFs in suspension for eight days to allow for the development of round EBs. At day eight, EBs were plated on gelatin-coated plates and cultured for an additional 25 days. During this time, cells grow out of the EBs while differentiating into all three germ layers. At days 0,8 , and $8+25$, samples were taken, RNA was isolated and differentiated cells were tested for expression of genes characteristic of all three germ layers with semiquantitative RT-PCR (Figure 5-23). MEFs were used as negative control.

\section{Crt-DCM $2.1 \quad$ Crt-DCM 2.2}

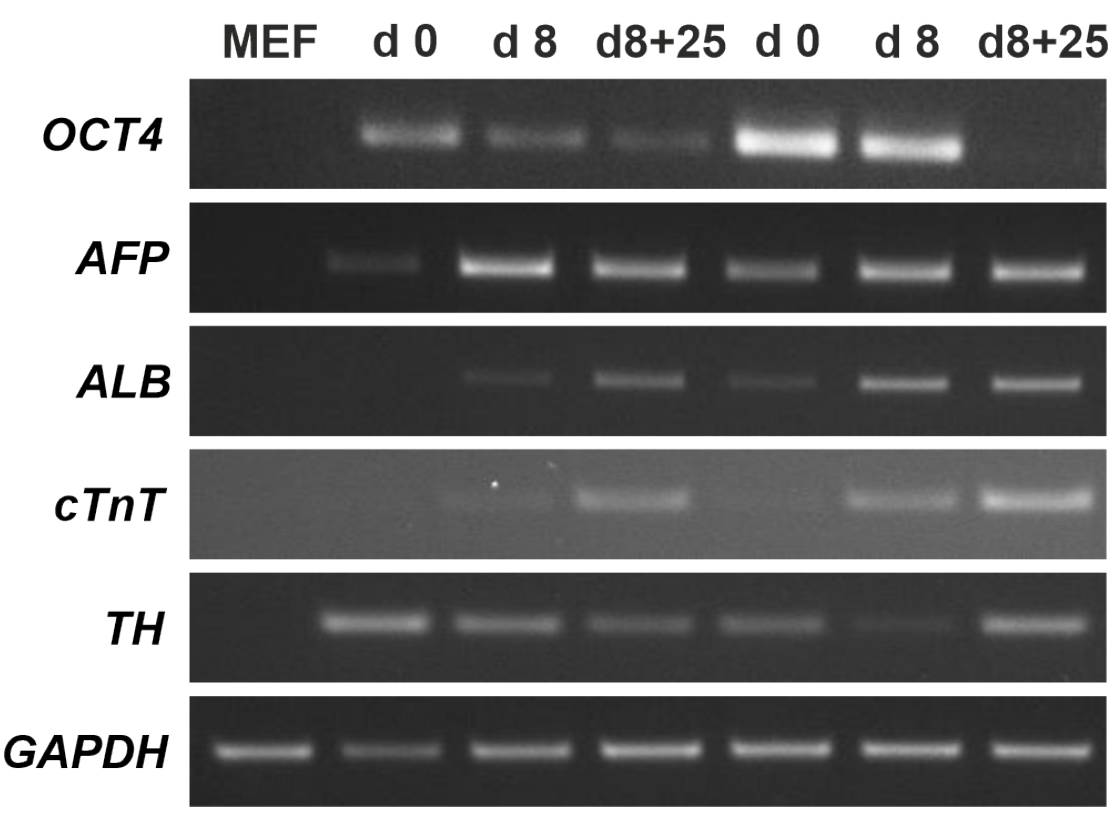

Figure 5-23: PCR results show expression of germ layer marker genes at three time points during spontaneous differentiation.

MEFs served as negative control. MEF = mouse embryonic fibroblasts.

RT-PCR results showed a decline in the expression of the stem cell marker Oct-4 in a time dependent manner in both cell lines tested (Crt-DCM 2.1 and Crt-DCM 2.2) with a remaining Oct-4 expression at time point $8+25$ in Crt-DCM 2.1 (Figure 5-23) suggesting a few cells were undifferentiated at that time point. Furthermore, in all cell lines the expression of endodermal markers, $A F P, A L B$, and a mesodermal marker, $c T n T$, increased with differentiation time. The ectodermal marker tyrosine hydroxylase $(T H)$ was already expressed in undifferentiated cells, which was unexpected. This signal might have come from cells that had spontaneously differentiated during stem cell culture. The clear expression of $\mathrm{TH}$ at day $8+25$ was according to our expectations. The expressions of these markers indicate that our iPSCs were capable of differentiating into cells of all three germ layers. 
Taken together, the cells derived from PBMCs of Crt-DCM or skin fibroblasts of DCM patient had a stem cell-like morphology, expressed characteristic stem cell markers on mRNA and protein level, and were capable of differentiating into all three germ layers, which leads to the conclusion that these cells are induced for pluripotency.

\subsubsection{Generation and characterization of iPSC-derived cardiomyocytes} To obtain a high number of pure cardiomyocytes, Crt-DCM and DCM-iPSCs were differentiated in cardiomyocytes as described before. iPSC-CMs were cultured for at least 60 days after onset of differentiation before they were used for experiments. Ten to 14 days after initiation of differentiation, the distinctive characteristic of contraction could be observed for the first time. To confirm the manifestation of cardiomyocyte cell structure, iPSC-CMs were stained for alpha-actinin (Figure 5-23 A, B) using immunocytochemistry. The 60-day-old Crt-DCM and DCM-iPSC-CMs had a heterogenous morphology from round to long-shaped cells (Figure 5-24). Alpha-actinin staining of Crt-DCM-iPSC-CMs showed a highly organized sarcomeric structure with clear striations. We observed also bi- and multinucleated cells in both cell lines.
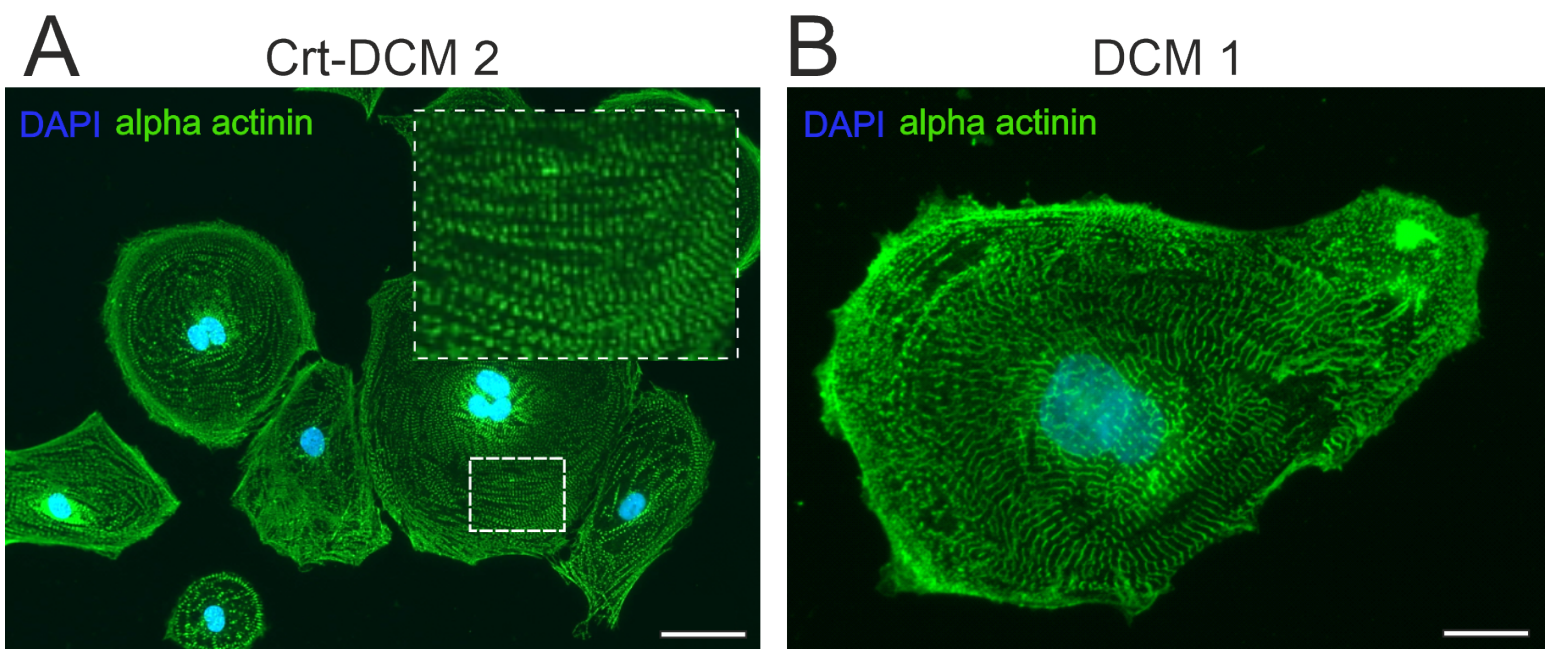

Figure 5-24: Immunofluorescence staining of alpha-actinin in iPSC-CMs.

Crt-DCM 2 iPSC-CMs (A) and DCM 1 iPSC-CMs (B) were stained for alpha-actinin (green) and DAPI (blue). Both cell lines showed characteristic cardiac structure with regular, striated alpha-actinin organization. Scale bars $=100 \mu \mathrm{m}(\mathbf{A})$ and $20 \mu \mathrm{m}$ (B).

\subsubsection{Cell size measurement}

DCM and hypertrophic cardiomyopathy ( $\mathrm{HCM}$ ) often occur in a mixed form. To determine if the generated DCM 1 iPSC-CMs differ in volume and size from control cells, we measured the cells with CASY Cell Counter \& Analyzer (OLS OMNI Life Science, Bremen, Germany). To gain a broader understanding of the relation of cell size, an additional iPSC line from a second DCM patient was added to the analysis (DCM 3). The DCM 3-iPSCs were independently generated and characterized in the lab of K. Streckfuß-Bömeke. 
Representative brightfield images of CRT 1-, DCM 1-, and DCM 3-iPSC-CMs showed that iPSC-CMs of the DCM 1 line were smaller compared to the other iPSC-CMs (Figure 5-25A). Results of the volume- and diameter measurements confirmed that iPSC-CMs of the DCM 1 patient were significantly smaller than iPSC-CMs from the control cell line (Figure 5-25B). DCM 3-iPSC-CMs were significantly larger than control and DCM 1-iPSC-CMs. The differences were consistent between volume measurement and diameter measurement (Figure 5-25B).

iPSC-CMs from DCM 1 patient 1 were significantly smaller than controls and cells of DCM patient 3 are significantly larger compared to control cells.

A

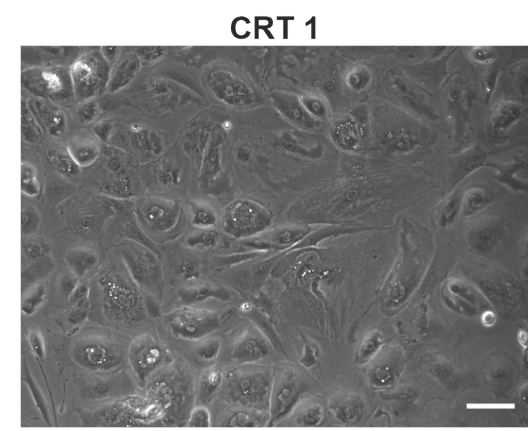

DCM 1

DCM 3
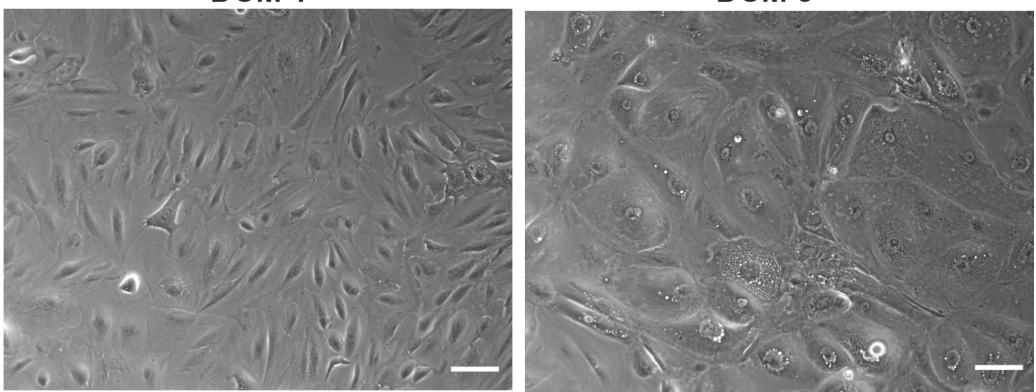

B
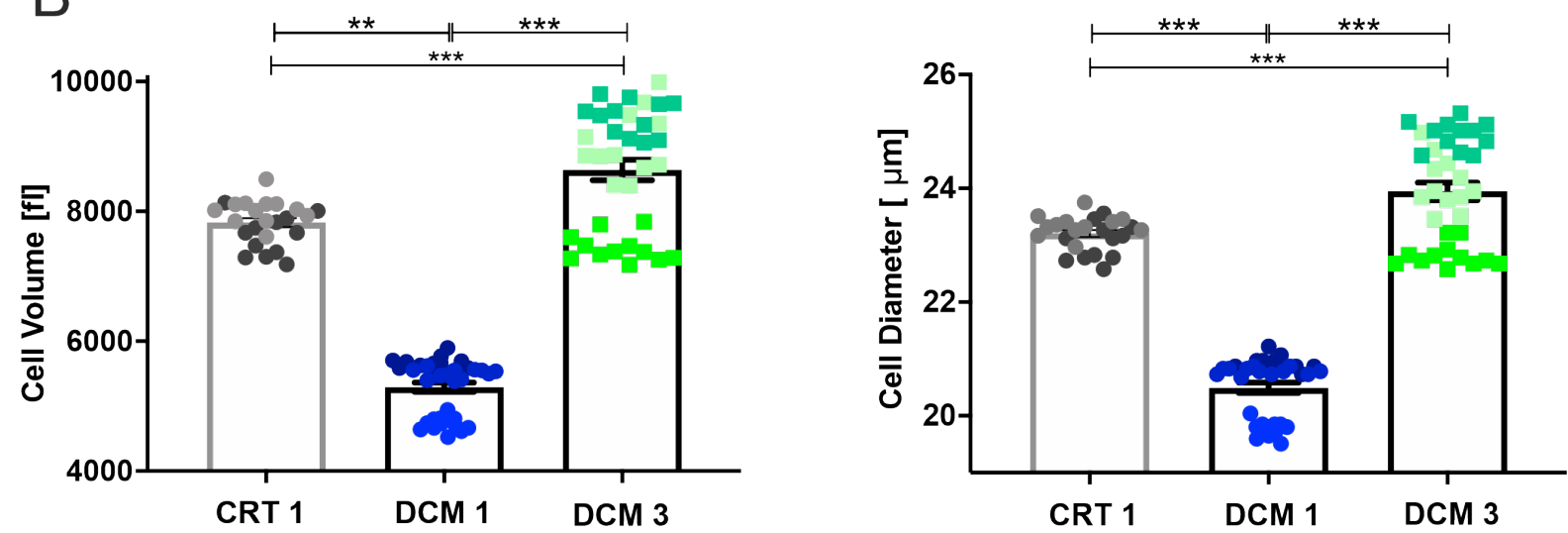

Figure 5-25: iPSC-CM volume and size measurements in 3D with a CASY cell counter.

A: Representative bright field images of iPSC-CMs of CRT 1, DCM 1 and DCM 3 in culture on the day of the CASY measurement. Scale bars $=20 \mu \mathrm{M}$. B: iPSC-CMs from DCM patient 1 (DCM 1) and DCM patient 3 (DCM 3) differed significantly in cell volume from control-iPSC-CMs (CRT 1). DCM 1 cells were significantly smaller than CTR 1 cells and DCM 3 iPSC-CMs were significantly larger. The three tested cell lines differed significantly in cell diameter. $n=$ average cell volume/diameter of approximately 6000 measured cells from one well of a 12-well plate. CTR 1: Two differentiations from one cell line. DCM cell lines: Three differentiations from one cell line, respectively. Statistical analysis was performed with one-way ANOVA. $P$ values were represented as followed: $p>0.05=* ; p$ $>0.01=* * ; p>0.005=* * *$. Bars indicate mean values $+/$ - SEM. 


\subsubsection{Regularity of sarcomeric structure}

A previous study from our group investigated the impact of DOX on the sarcomeric regularity of iPSC-CMs from ACT patients, which were also used for this study (Haupt, 2018). It was shown that sarcomeric regularity in ACT-iPSC-CMs after DOX treatment is reduced and that the reduction is greater in ACT-iPSC-CMs compared to control iPSC-CMs (Haupt, 2018). While ACT and DCM patients shared a common disease phenotype, the main difference between these cardiomyopathies is the trigger of the disease. Therefore, we were interested if effects of DOX on the sarcomere are universal in diseased iPSC-CMs or if the susceptibility is specific to ACT-iPSC-CMs.

Sarcomeres are the smallest functional units of myofibrils. Lesions in these units can have detrimental effects on force development and contractile function (Eschenhagen et al., 2015). In this study, we wanted to investigate whether the previously described reduction in sarcomeric regularity after DOX treatment can also be observed in end-stage heart failure DCM-iPSC-CMs. In this way we can test whether this phenomenon is common in heart failure, specific to DOX-predisposed patients or based on certain genetic backgrounds. To assess sarcomeric integrity, we tested the regularity of sarcomeres in our iPSC-CMs with immunocytochemistry. Application of FFTA on the microscopy images allowed for a qualitative assessment of regularity of the observed structures and therefore for an objective evaluation of sarcomeric structures in patient and control cell lines. 
untreated

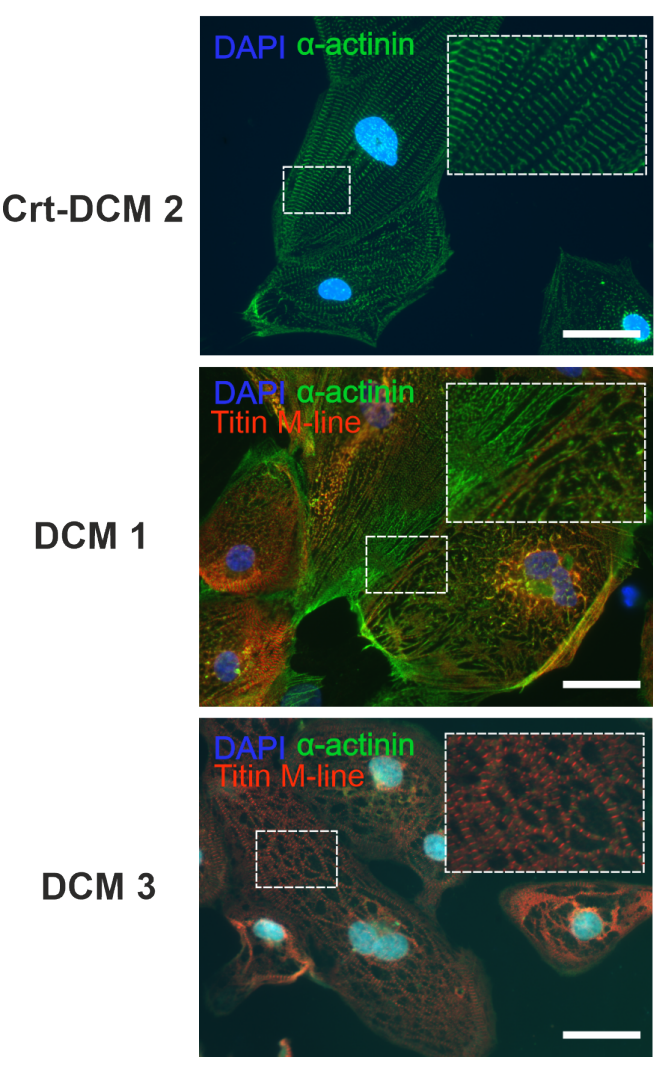

$0.25 \mu \mathrm{M}$
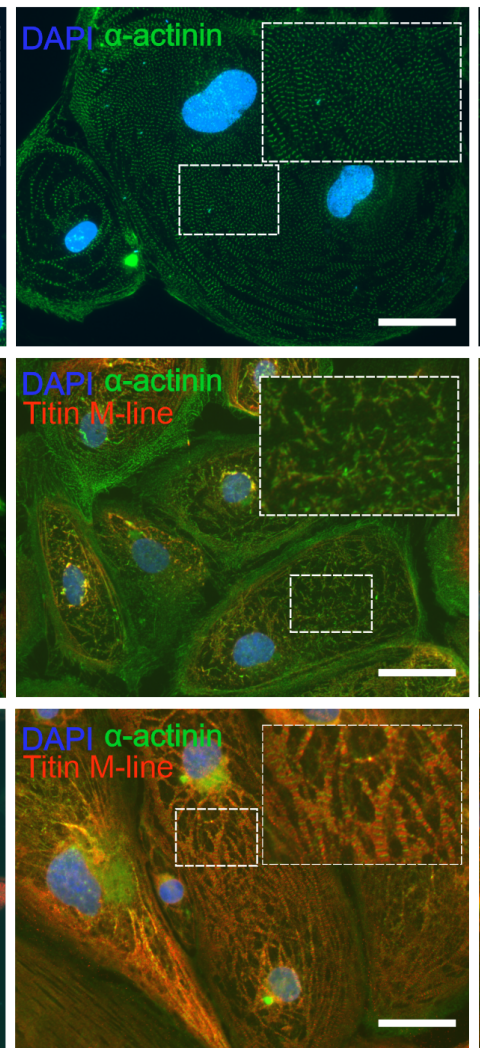

$1 \mu \mathrm{M}$
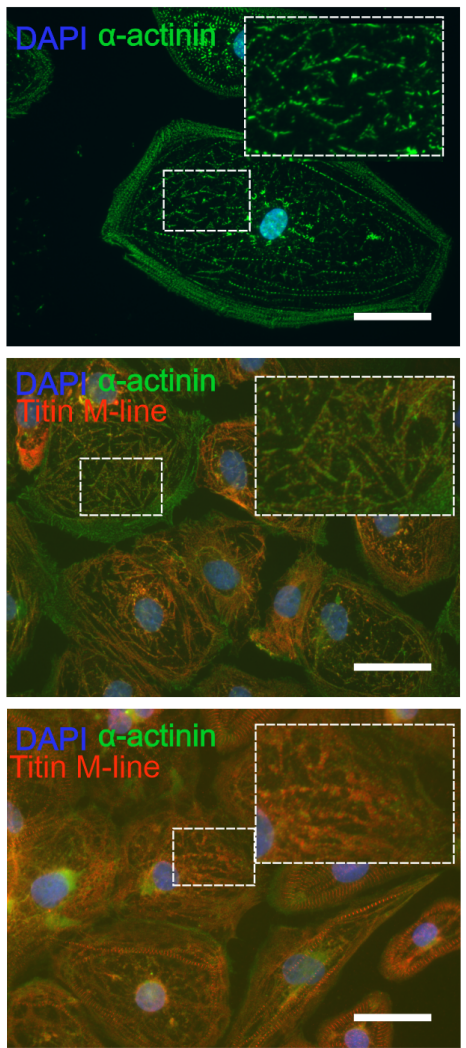

Figure 5-26: Representative $\alpha$-actinin immunofluorescence images of DOX-treated iPSC-CMs and untreated iPSC-CMs.

Cells were stained for alpha-actinin and/or titin. Scale bars $=100 \mu \mathrm{m}$.

Representative images demonstrated regular alpha-actinin structures in untreated Crt-DCM cells, whereas the sarcomeric regularity is impaired upon DOX treatment at low and high concentrations as seen in the $\alpha$-actinin staining (Figure 5-26 upper panel). In DCM 1- and DCM 3-iPSC-CMs, regularity of sarcomeric structures was already impaired without DOX treatment (Figure 5-26). In addition, DOX treatment worsened the impairment in sarcomeric structures of the cells.

Quantification of sarcomeric regularity confirmed the trend seen in the immunofluorescent images, namely that DOX treatment was detrimental to sarcomeric regularity (Figure 5-27A). The DOX-induced decrease in sarcomeric regularity was most prominent in Crt-DCM cells and less clear in iPSC-CMs from DCM 1 and DCM 3 (Figure 5-27A, B). This is in line with the observed reduction in sarcomeric regularity of ACT-iPSC-CMs after DOX treatment (Haupt, 2018). Among all three tested conditions, Crt-DCM-iPSC-CMs showed significantly higher sarcomeric regularity than iPSC-CMs from DCM 1 and DCM 3 (Figure 5-27C). DOX treatment had detrimental effects on sarcomeric regularity in healthy and diseased iPSCCMs. When sarcomeres were intact as in the Crt-iPSC-CMs, the observed DOX effect was more prominent. In contrast, in the DCM 1 and DCM 3-iPSC-CMs, the sarcomeres displayed 
low structural regularity under untreated conditions, and therefore the effect through DOX treatment was small.

Analysis of sarcomeric regularity in the iPSC-CMs from DCM patients showed that treatment for $24 \mathrm{~h}$ with $1 \mu \mathrm{M}$ DOX leads to more irregular sarcomeric structure in iPSC-CMs from CrtDCM, DCM 1, and DCM 3. The most significant decrease of sarcomeric regularity after DOX treatment was detected in Crt-DCM-iPSC-CMs. In iPSC-CMs of DCM 1 and DCM 3, the observed decrease of sarcomeric regularity after DOX treatment was minimal, likely due to highly disorganized sarcomeres before DOX treatment. 
A

Crt-DCM 2

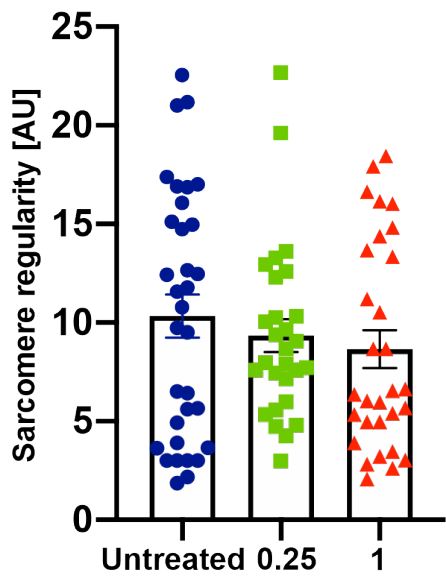

Doxorubicin $[\mu \mathrm{M}]$
DCM 1

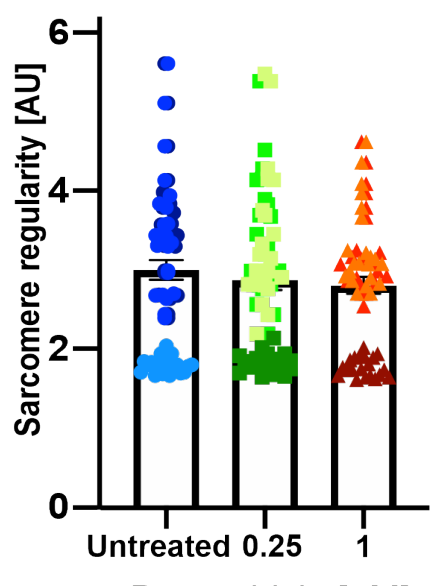

Doxorubicin $[\mu \mathrm{M}]$
DCM 3

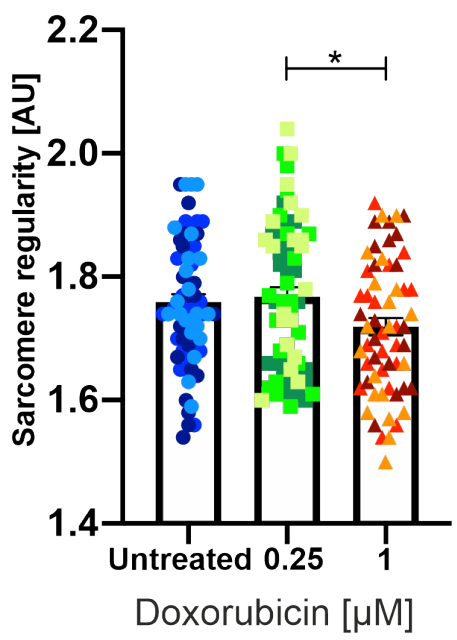

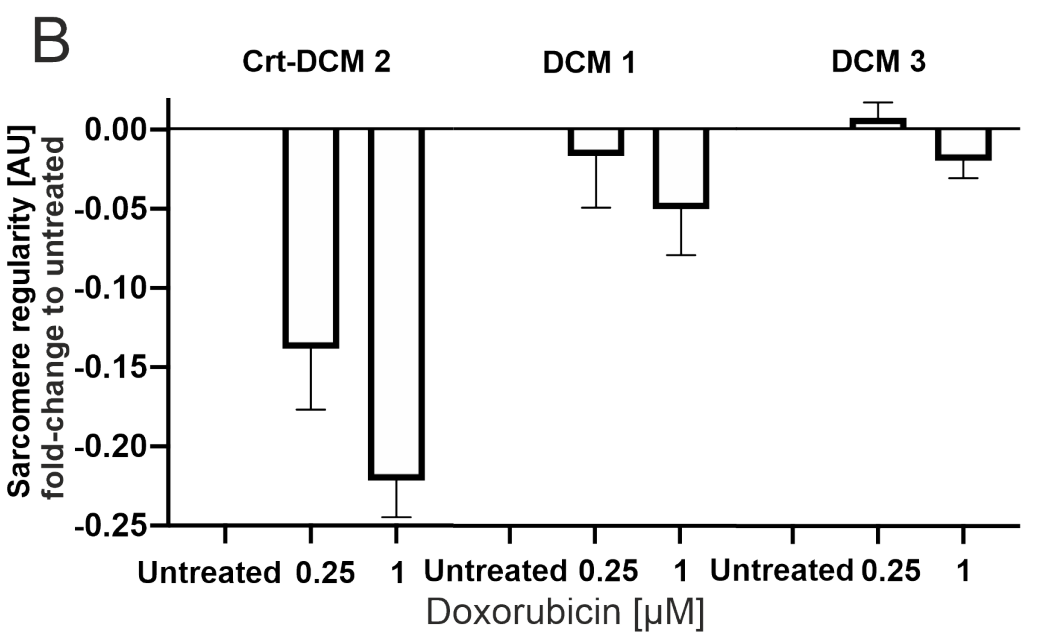

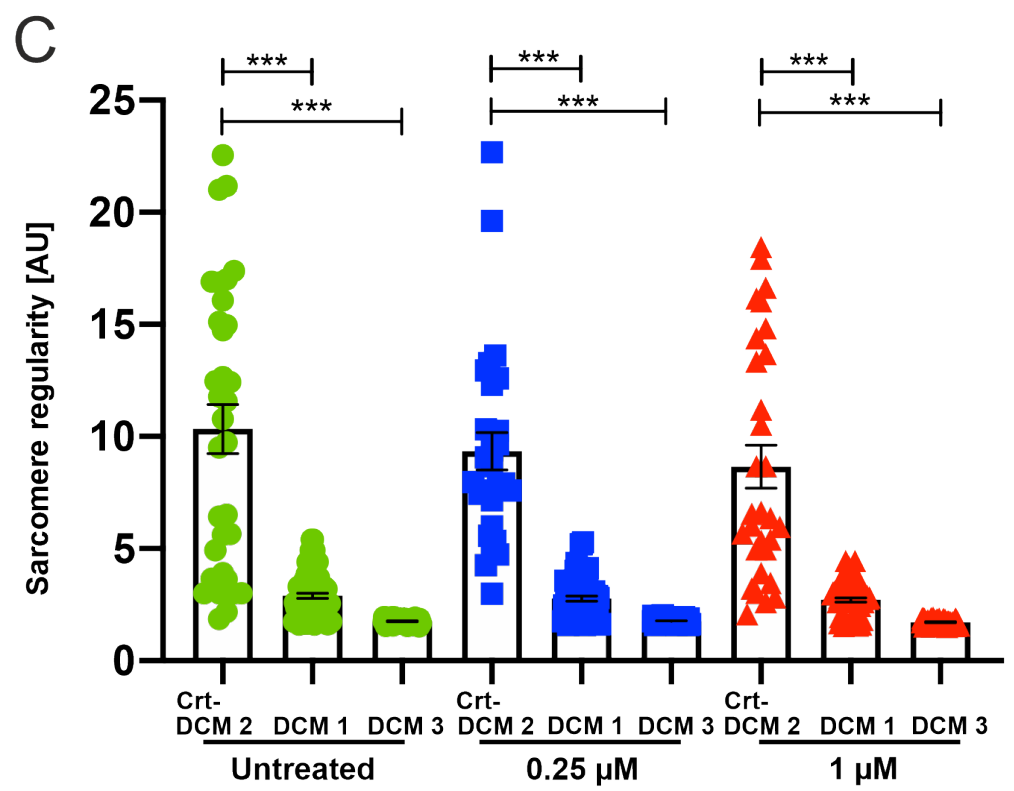

Figure 5-27: Integrity of the sarcomeric structure in iPSC-CMs of different donors.

A: Sarcomeric regularity in iPSC-CMs from Crt-DCM 2, DCM 1 and DCM 3. DOX treatment reduced the sarcomeric regularity in all cell lines. B: DOX-induced relative change in sarcomeric regularity of alpha-actinin-stained iPSC-CMs of Crt-DCM 2, DCM 1 and DCM 3. C: Comparing all three cell lines, sarcomeric regularity is significantly reduced in iPSC-CMs from DCM 1 and DCM 3 compared to iPSC- 
$C M$ of Crt-DCM 2. The significant difference applies to all treatment conditions. $A U=$ arbitrary unit. $n$ = calculated regularity from one microscopy image. Crt-DCM 2: $n=28-33$ images of cell from one differentiation. DCM 1 and DCM 3: $n=61-68$ images from three independent differentiations from one cell line, respectively. Statistical analysis was performed with one-way ANOVA. Multiple comparisons were corrected according to Tukey. $P$ values were represented as followed: $p>0.05=*$; $\mathrm{p}>0.01=* * ; \mathrm{p}>0.005=* * *$. Bars indicate mean values $+/$ - SEM.

\section{Discussion}

\subsection{Uptake, retention, and distribution of DOX in iPSC-CMs}

Accumulative doses of DOX are the most reliable predictor of ACT development (Lefrak et al., 1973; von Hoff et al., 1979; Swain et al., 1997; Lotrionte et al., 2013; Ewer and Ewer, 2015). Although DOX has been long known to cause cardiac myopathies, the exact mechanisms have not been unraveled (Tripaydonis et al., 2019). DOX was shown to bind to mitochondrial cardiolipin extracted from rats in an experimental set-up with cardiolipincontaining monolayers (Goormaghtigh et al., 1990). Binding of DOX and its subsequent reduction to a semiquinone damages the mitochondrial membrane (Mordente et al., 2001). This damage interrupts the electron transport chain and lets electrons leak out into the cytoplasm, causing elevated levels of ROS (Mordente et al., 2001). This effect was shown in rabbit, rat, mouse, pig, monkey, and dog models (Herman and Ferrans, 1997; Robert, 2007; Lamberti et al., 2014). Furthermore, DOX-induced damage was reported in human cells in vitro, with a greater effect in relatively mature iPSC-CMs of 60 days compared to 30-day-old iPSC-CMs (Cui et al., 2019). Studies in mouse and rat models revealed that activation of autophagy and mitophagy following DOX treatment protects from cardiotoxicity (Mitra et al., 2007; Kawaguchi et al., 2012; Hoshino et al., 2013; Xu et al., 2013; Dutta et al., 2014). Hence, dysregulation of endogenous autophagy and mitophagy through DOX are likely to contribute to cardiotoxicity (recently reviewed by Koleini and Kardami, 2017). Furthermore, DOX increases cellular ROS levels by so-called redox cycling (Davies and Doroshow, 1986). DOX is also known to induce DNA double-strand breaks, damaging cellular and mtDNA. A study that used 8-hydroxydeoxyguanosine as a biomarker for DNA damage in rats reported that mtDNA in the heart was affected more by DOX than mtDNA in the liver. Furthermore, mtDNA damage in the heart was twice as severe as nuclear DNA damage in the heart (Serrano et al., 1999). This effect was also prominent in a mouse model (Adachi et al., 1993), and was reported in a model of long-term cardiotoxicity (Hixon et al., 1981). Mitochondria with faulty DNA cannot replicate efficiently. Therefore, mtDNA damage is thought to contribute mainly to the development of late-onset, chronic cardiotoxicity (Wallace, 2003; Lebrecht and Walker, 2007).

Until now it has not been clearly determined how DOX increases ROS, where in the cell it binds, how it triggers long-term toxicity, and where in the cell most DOX accumulates. With this knowledge, it may be possible to limit the cardiotoxic effects of DOX and ultimately 
prevent ACT, without hampering its anti-neoplastic properties. So far, there has not been a study that methodically quantified intracellular DOX levels in human cardiomyocytes. In this study, we used HPLC to analyze the amounts of DOX in IPSC-CMs of ACT patients and control patients after DOX treatment. We varied DOX concentrations, treatment times, and recovery periods and found that intracellular DOX levels positively correlate with DOX concentration and with treatment time. Furthermore, these data show that intracellular DOX levels decrease significantly after a recovery period of three days in control iPSC-CMs and seven days in ACT-iPSC-CMs.

Initially, it was important to establish the HPLC DOX detection approach for biological samples such as iPSC-CMs. We used an adaption of a previously described protocol to measure DHE for ROS analysis (Fernandes et al., 2007). HPLC had been used formerly to determine DOX levels in aqueous solutions (Wood et al., 1990), rat serum, and homogenized lung tissue from pigs (Kümmerle et al., 2003).

Our data show a correlation between the concentration of DOX treatment and intracellular DOX levels. In the tested range up to $5 \mu \mathrm{M}$ DOX, iPSC-CMs did not saturate with DOX. In ACT-iPSC-CMs, intracellular DOX levels were twice as high in samples treated with $1 \mu \mathrm{M}$ DOX compared to $0.5 \mu \mathrm{M}$ DOX. Five times higher DOX concentrations (from $1 \mu \mathrm{M}$ to $5 \mu \mathrm{M}$ ) also resulted in a five-fold increase of intracellular DOX (Figure 5-1A). In a limited number of differentiation experiments, we found higher absolute DOX levels in ACT patient iPSC-CMs compared to control under nearly all conditions (Figure 5-1). This finding requires confirmation using data from more independent differentiation experiments.

It was reported over 40 years ago that DOX enters Ehrlich ascites tumor cells via diffusion (Skovsgaard, 1977). For the lipophilic DOX molecules, cell membranes do not constitute a hurdle (Tripaydonis et al., 2019). In line with these previous findings, there seems to be little regulation by the cell membrane on how much DOX enters iPSC-CMs. In the present study, it was shown that in both the ACT patient and control patient groups, DOX levels in samples treated with $5 \mu \mathrm{M}$ DOX were about five times higher than in samples treated with $1 \mu \mathrm{M} D O X$ (Figure 5-1). Therefore, potentially saturating DOX concentrations have to be higher than 5 $\mu \mathrm{M}$. In cancer patients, DOX concentrations in blood plasma only exceed $5 \mu \mathrm{M}$ for few minutes right after DOX infusion (Greene et al., 1983). If recommendations regarding prolonged infusion of DOX with reduced flow are being followed, saturation might not be reached in clinical settings (Legha et al., 1982; El-Kareh and Secomb, 2000). DOX levels in iPSC-CMs from ACT patients tend to be higher than in control patients. If this difference is due to increased resorption of DOX in ACT patients compared to control patients or if it is due to a difference in efflux efficacy between the two groups remains undetermined. It is unclear whether diffusion is the only mechanism for DOX resorption or whether importers or 
exporters are involved. The SNP rs8187710 in the ATP-binding cassettes subfamily C member 2 (ABCC2) is one of only three SNPs that significantly increased the risk of cardiotoxicity in patients treated with anthracyclines indicating the possible involvement of $A B C$ transporters in $A C T$. $A B C C 2$ is part of a superfamily of transport proteins that transport substrates across the cell membrane in an ATP-dependent manner (Elens et al., 2011). Occurrence of the rs8187710 SNP has been shown to reduce ATPase activity and cause a reduced efflux activity due to an amino acid change in the mRNA sequence (Elens et al., 2011). However, no experimental data exist on the prevention of ACT by rs 8187710 .

For time course experiments, iPSC-CMs were treated with $1 \mu \mathrm{M}$ DOX for up to $72 \mathrm{~h}$ and intracellular DOX values were measured after $0.5,2,6,24,48$, and $72 \mathrm{~h}$ without recovery time between treatment and harvest. We found that treatment time determines intracellular DOX content. Intracellular DOX was already detectable after $0.5 \mathrm{~h}$ of treatment and continued to increase up to $48 \mathrm{~h}$. In ACT-iPSC-CMs, intracellular DOX levels after $48 \mathrm{~h}$ were increased 4.5 -fold compared to the $0.5 \mathrm{~h}$ time point. In the same period, intracellular DOX levels of control iPSC-CMs increased 11.6-fold (Figure 5-2A). Levels between $6 \mathrm{~h}$ and $24 \mathrm{~h}$ treatment time only differed slightly in both groups (ACT and control; Figure 5-2). The lower values at the $72 \mathrm{~h}$ time point can be explained by increased cellular stress and subsequent apoptosis. This hypothesis is underlined by our former studies with these cells, where we showed that the apoptosis rate after $24 \mathrm{~h}$ of $1 \mu \mathrm{M}$ DOX treatment was around $15 \%$ and had doubled after $72 \mathrm{~h}$ of $1 \mu \mathrm{M}$ DOX treatment (Haupt, 2018). Also, Burridge and colleagues reported an apoptosis rate of $55 \%$ in iPSC-CMs that were treated with $1 \mu \mathrm{M}$ DOX for $72 \mathrm{~h}$ (Burridge et al., 2016). To compare intracellular DOX levels for treatment times beyond $48 \mathrm{~h}$, it would be necessary to normalize the HPLC values to cell numbers at sample harvest. In conclusion, our results show that DOX enters iPSC-CMs immediately and that intracellular DOX levels increase over time.

Studies on DOX concentrations in plasma during treatment suggest rapid clearance of the drug from the circulation (Greene et al., 1983). It has not been assessed if DOX molecules remain in human cardiomyocytes for extended periods after treatment. In our work, we treated iPSC-CMs with $1 \mu \mathrm{M}$ DOX for $24 \mathrm{~h}$ and then allowed the cells to recover for 3 and 7 days. Our results demonstrate that three days after treatment, intracellular DOX levels were about $50 \%$ lower than immediately after treatment, and after seven days, DOX levels were close to the detection limit in both groups (Figure 5-3). Normalized values show that intracellular DOX levels were lower in the control patient group after three days and in the ACT patient group after seven days. The clearance rate in the iPSC-CMs from ACT patients may be lower than in cells from control patients. 
One limitation of the recovery assay is that other sources of DOX degradation cannot be excluded. Degradation of DOX in water can be driven by ambient light (Racles et al., 2019). Although later studies claim that DOX is photostable, it is accepted that light can trigger oxidation- and catalyst-driven breakdown (Wood et al., 1990; Kaushik and Bansal, 2015). Therefore, exposure to ambient light was avoided as much as possible. While our samples were shielded from light in the incubators, the abundance of oxygen might have reduced DOX levels. Furthermore, iPSC-CMs were cultured at $37^{\circ} \mathrm{C}$ in full cardio culture medium, including antioxidants, which could promote DOX degradation. DOX is stable at a $\mathrm{pH}$ value of 7 , with significantly decreasing stability in solutions with higher or lower $\mathrm{pH}$ value. We used a Tris-based lysis buffer with a $\mathrm{pH}$ value of 7.4. Additionally, samples were washed and resuspended with PBS, also using a $\mathrm{pH}$ of 7.4. During handling, samples were kept on ice, covered with tin foil, and when lined up for measurement, HPLC samples were shielded from light and cooled to $4^{\circ} \mathrm{C}$. These measures were consistently applied to reduce DOX breakdown. Hence, HPLC sample preparation from our iPSC-CMs was comparable to the described preparation performed by Kümmerle et al. (2003). Normalized recovery data show that a significant reduction of intracellular DOX is reached after three days in control iPSCCMs, while it takes seven days to reach a significant decrease in ACT-iPSC-CMs (Figure $5-3 B)$.

\subsection{Intracellular distribution of DOX}

During membrane isolation, we noted that the mito fraction pellet of DOX-treated cells had a strong red coloration. Since this coloration was only present in pellets from DOX-treated cells, it was likely that it stemmed from the remaining drug, which would be in line with findings that DOX binds to mitochondrial cardiolipin (Goormaghtigh et al., 1980; Aryal and Rao, 2016). To test this hypothesis with quantifiable data, iPSC-CMs from ACT patients and control patients were treated with $1 \mu \mathrm{M}$ DOX for $24 \mathrm{~h}$ and then immediately lysed. The lysates were fractionated with differential ultracentrifugation, which resulted in three distinct cell fractions: the first fraction with mitochondria and nuclei, a fraction with cellular membranes, and the remaining plasma membrane fraction. To produce the fractions, centrifugation was preferred over commercial fractionation kits (for example "Subcellular Protein Fractionation Kit for Cultured Cells", Thermo Scientific), because these kits use different lysis buffers with different $\mathrm{pH}$. In our approach, the $\mathrm{pH}$ was consistent throughout the fractions. Acetonitrile was added to the fractions to dissolve DOX before samples were prepared for HPLC measurement.

We found that more than half of the total DOX was detected in the mito fraction in both ACT and control. The DOX levels in the mito fractions were significantly higher compared to the membrane fractions (Figure 5-4, appendix). The mean DOX amount was insignificantly higher in ACT-derived cells as compared to controls (Figure 5-4). Knowing that DOX has a 
high affinity for mitochondrial cardiolipin (Nicolay et al., 1984; Goormaghtigh et al., 1990; Aryal and Rao, 2016), this result was expected. Generally, the pellet size of the mito fraction was considerably larger than the one of the membrane fractions. Therefore, protein concentrations of the fractions were determined with a Bradford assay and HPLC values were normalized to the respective protein concentrations. Comparing normalized values, the distribution of DOX did not differ between the three fractions. In the control patient group, the mito fraction still had the highest amount of DOX, referred to the total amount in control iPSC-CMs. Looking at fractions of total DOX content in the ACT patient group, DOX values were higher in the membrane fraction and the cytoplasm compared to the mito fraction. This was contrary to our hypothesis, where binding affinity of DOX would suggest that even when normalized to protein content, most DOX would be bound to cardiolipin and hence associated with the mito fraction. Escribá et al. (1995) reported that Daunorubicin, an anthracycline drug like DOX, directly influences levels of membrane-associated $G$ proteins and protein kinase $\mathrm{C}$-alpha beta in the heart. This indicates a direct interaction with membrane proteins. More recently, it was shown in model membranes that DOX location in membranes is mediated by non-covalent interactions such as electrostatic, hydrophobic, and van der Waals interactions (Alves et al., 2017). However, the authors did not specify if that results in a high or low probability of DOX retention in the membrane.

Our findings do not contradict previous observations that DOX has a high affinity for mitochondria. It could be that when cells are challenged with a relatively high dose of DOX as $1 \mu \mathrm{M}$, it binds to certain proteins with high affinity, but due to high plasma levels, it also binds to other proteins in a nonspecific manner. To test this hypothesis, this experiment could be repeated either with lower DOX concentrations or with a recovery period of 24 to $48 \mathrm{~h}$. Measuring DOX distribution after a short recovery period would provide additional information about whether DOX is cleared simultaneously from all cell compartments, or if there are cellular areas in which DOX is retained longer than in others.

Although sample preparation was always done consistently, it should be noted that part of the samples were prepared at the University Hospital Göttingen and others at King's College London. This circumstance could partially explain the variance of data points in Figure 5-1. Another limitation could have been the temporal distance between the measurements because HPLC values depend to some extent on the age of the column and the power of the laser; both of which change over time. Data points from the subcellular distribution experiments (Figure 5-4) were all prepared in one location and measured within a few weeks of each other. They show a smaller variance, which is in line with the described limitation. Nevertheless, the data in Figure 5-1, Figure 5-2, and Figure 5-3 show a clear trend, which might be more prominent when the above mention measures were applied. 
Here we show for the first time the resorption of DOX in human iPSC-CMs. These results provide evidence that DOX molecules do not remain in cardiac cells for extended periods. This implies that long-term cardiotoxicity is driven by impairments that were caused by an initial DOX interaction in a hit-and-run manner.

\subsection{DOX-induced translational decrease in iPSC-CMs}

In gene expression experiments with the described ACT- and control IPSC-CMs, many genes encoding for proteins that compose sarcomeric structure or calcium homeostasis were down-regulated after DOX treatment (Haupt, 2018). To test if this effect would also be seen on the translational level, global mRNA translation in iPSC-CMs after DOX treatment was tested with the surface sensing of translation (SUnSET) method (Schmidt et al., 2009). This approach is nonradioactive, quantifiable, and does not require any pretreatment of the cells. In brief, puromycin, a structural analog of aminoacyl transfer RNAs, is integrated into forming polypeptide chains, which terminates translation. Blocked ribosomes release puromycinmarked polypeptide chains, which in turn accumulate and can be detected with a puromycin antibody. The amount of puromycin signal correlates directly with global mRNA translation. Although this method might have its limitation when used to analyze glucose-starved cells during metabolic selection (Marciano et al., 2018), it worked well in the iPSC-CM model with full cardio culture medium.

In this assay, iPSC-CMs were treated with $1 \mu \mathrm{M}$ DOX for $24 \mathrm{~h}$, which led to a significant reduction in mRNA translation. This trend could be detected in all patients and control cell lines and was significant in both the ACT patient and the control patient groups. The effect of DOX treatment on mRNA translation was comparable in both groups, with a $20 \%$ reduction after $24 \mathrm{~h}$ of $1 \mu \mathrm{M}$ DOX treatment. Our results are in between previously reported rates, where the translation was reduced after $24 \mathrm{~h}$ with $1 \mu \mathrm{M}$ DOX treatment by only $2 \%$ in human keratinocytes (Sauter et al., 2010) and by $75 \%$ in prostate cancer cells (White et al., 2007). White et al. (2007) reported that decreased translation was caused by sustained phosphorylation of elongation factor 2 (EF-2). Elevated ROS levels trigger kinaseindependent phosphorylation of EF-2, which obstructs protein elongation (White et al., 2007). Additionally, in a genome-wide integrative systems biology approach in yeast, it was shown that DOX treatment negatively affected several pathways related to ribosome biogenesis and mRNA translation (Taymaz-Nikerel et al., 2018).

Having established that DOX reduces the global mRNA translation rate, we were also interested if transcription is decreased globally. Therefore, we performed whole transcriptome analysis with next generation sequencing of generated 3D-engineered heart muscle (EHM) from ACT and control iPSC-CMs after DOX treatment. We identified 1106 upregulated differentially regulated genes (DEGs) and 990 down-regulated DEGs in both ACT 
patient and control EHMs after DOX treatment (unpublished data from our group). GO enrichment analysis of DOX-induced DEGs in both control and ACT-EHMs identified significantly increased expression of genes with key functions including programmed cell death (e.g. BCL2L1, FAS, BAX) or regulation of catabolic processes (e.g. STAT3, ABHD5). Furthermore, we found significant decreases in the expression of actin cytoskeletal organization (e.g. ACTA2, MYBPC3, MYH6, MYH7, MYL2, MYL7, TNNI3, TNNT2), muscle contraction (e.g. LDB3, MYBPC3, MYH6, MYL2, MYOM1, SORBS1, STMN1, TCAP, TNNT2), and chromosome organization $(2.19 \%, B R C A 2, T O P 2 A, T O P 2 B)$ in both groups after DOX treatment (data not shown). This shows that while several genes have decreased transcription after DOX treatment, other genes are up-regulated, or not changed at all. Overall, transcription is changed in a much more differentiated way than mRNA translation. Hence, reduced translation after DOX treatment is not a direct result of decreased transcription but must have transcription-independent causes.

mRNA translation initiation is mainly regulated through a conserved mechanism, involving the phosphorylation of the initiation factor elF2 $\alpha$, which is a downstream actor in the mechanistic target of rapamycin (mTOR) pathway (Wang and Proud, 2006). This pathway is a major regulator in cellular metabolism, growth, maintenance of homeostasis, and autophagy (Zhao et al., 2015; Manning and Toker, 2017; Sciarretta et al., 2018a, 2018b). As such, it is a highly interesting target to study in ACT because there have been reports about ROS regulation through the mTOR pathway. Increased mTOR signaling results in increased Nox2 mRNA levels and elevated ROS in endothelial cells (Reho et al., 2019). Also, growth factor-induced ROS in T-cells is mediated through mTOR signaling and NOX activity (Silva et al., 2011). Inhibition of mTOR signaling through DOX treatment was reported to induce cardiac dysfunction through p53 (Zhu et al., 2009).

To gain a complete understanding of the interplay between DOX damage and mTOR signaling, it would be worthwhile to consider another downstream target of mTOR signaling: autophagy. Autophagy is a cellular mechanism in which dysfunctional components are engulfed, degraded, and recycled. Autophagy and its mitochondrial form, mitophagy, are greatly affected by DOX treatment (reviewed by Bartlett et al., 2017). Restoration of autophagy by regulating the AMP-activated protein kinase (AMPK)/mTOR pathway had ameliorating effects on DOX damage in rat cardiomyocytes and a mouse model (Cao et al., 2017). Recently, it was shown that ROS-induced autophagy is inhibited through NF-E2related factor 2 during extended periods of oxidative stress (Kosztelnik et al., 2019). This inhibition is facilitated through delayed down-regulation of AMPK expression.

Even a few years ago the understanding of autophagy was very limited (reviewed by Shintani and Klionsky, 2004). Today, it is known that autophagy is connected to many physiological 
and pathological processes. Autophagosome flux, or autophagic flux, has been described as a quantitative measure of the flow rate of material along the autophagosome pathway (Loos et al., 2014; Galluzzi et al., 2017). With the recently developed innovations in the quantification of autophagic flux, there will be advances in the characterization of autophagy in the development and progression of cardiac diseases (Yoshii and Mizushima, 2017; du Toit et al., 2018). Since NADPH oxidases regulate autophagy in cardiomyocytes, our ACT model with a distinct RAC2-related genetic background is a promising tool to unravel affected pathways in DOX-induced cardiotoxicity (Sciarretta et al., 2015). The findings of Lee et al. (2019) underline the importance of Nox2 regulation in cardiac health. They showed that exercise can restore auto- and mitophagy and down-regulate NOX2 in mice, and hence has a cardioprotective effect even after DOX treatment. It has not been investigated if restoration of autophagy is directly mediated through mTOR signaling, and if mRNA translation would be rescued as well. Our iPSC-CMs provide a platform to investigate these processes in a human model.

Taken together, our findings on the reduction of mRNA translation through DOX treatment and previous reports on DOX effects on oxidative stress, autophagy, and the Akt/mTOR pathway suggest that DOX influences fundamental cellular processes. It is still unknown to which extent the described changes are causes or consequences of the detrimental effects of DOX treatment. This iPSC-CM model is a valuable tool to research the molecular mechanisms behind ACT development in human iPSC-CMs with an ACT-specific genomic background.

\subsection{Gene editing with CRISPR/Cas9}

Clinical studies have established a linkage between a SNP in the RAC2 gene, which encodes for an activator of the Nox2 enzyme complex, and the development of ACT after DOX treatment (Wojnowski et al., 2005; Reichwagen et al., 2015). Although the RAC2 SNP rs13058338 is located in an intronic region, the occurrence of the SNP has been associated with higher mRNA expression of RAC2 and also of the Nox2 subunit p40phox (encoded by NCF4) in granulocytes (Schirmer et al., 2008). Increased activity of Rac2 would result in elevated superoxide levels through Nox2 activity. These findings can help to predict the risk of ACT development before the start of treatment. To gain a deeper understanding, it is necessary to unravel the molecular mechanisms underlying this linkage. The recent advancement of gene-editing tools, in particular CRISPR/Cas-based techniques, made it feasible to delete, insert, or swap gene fragments precisely and reliably (Gasiunas et al., 2012; Jinek et al., 2012; Cong et al., 2013). The most elegant way to determine if the abovementioned SNP contributes to the phenotype at all, is to correct the SNP in our ACT patient cell lines and compare the newly established cells with ACT patients and control patients. 
In our laboratory, gene editing with CRISPR/Cas9 in electroporated iPSCs has resulted in two iPSC lines with homozygous and ten iPSC lines with heterozygous corrections in the RAC2 SNP rs13058338. Separation and detailed analysis of mixed clones is likely to result in more iPSC lines with a SNP-corrected genotype.

The company that provided the reagents for gene editing suggested using an HDR template enhancer to increase the integration frequency. We tested the HDR enhancer with two different concentrations, namely $20 \mu \mathrm{M}$ and $30 \mu \mathrm{M}$. High concentrations of the HDR enhancer can be cytotoxic because it is dissolved in DMSO. Cytotoxicity was estimated by evaluating bright field microscopy images by cell density, amount of cell debris, and confluency. The HDR enhancer clearly showed a dose-dependent cytotoxic effect, which was greater than the stress of electroporation alone. Cell survival was less relevant than HDR template integration frequency, because only few cells with desired editing results are necessary to establish new cell lines. Therefore, it could be possible that the success rate was high, although fewer cells survived the procedure.

Amplicon sequencing gave a first indication of how many homozygous edits occurred. Electroporation without HDR enhancer resulted in an integration frequency of $6.6 \%$. It increased to $9.1 \%$ with $20 \mu \mathrm{M}$ HDR enhancer and was at $10.0 \%$ with $30 \mu \mathrm{M}$ HDR enhancer. Judging from the estimate given by amplicon sequencing, the HDR enhancer has a dosedependent benefit on integration frequency.

Sanger sequencing of individual clones allowed for a more reliable assessment of integration frequency. This showed that the use of an HDR enhancer did in fact increase the number of clones with edits from six without enhancer to ten and nine with $20 \mu \mathrm{M}$ and $30 \mu \mathrm{M}$ HDR enhancer, respectively. For the majority of these edited clones (six out of ten with $20 \mu \mathrm{M}$, and six out of nine with $30 \mu \mathrm{M}$ ), Sanger sequencing results do not allow a concluding remark about editing success, because the results were inconclusive. This could either stem from mixed clones, which happens when picked colonies did not stem from one clone alone. Or the genotype derived from an increased number of cut-repair-cycles at the target site. To further investigate why so many clones show ambiguous genotypes, the clones in question were separated and sequenced again.

To this point, the overall editing rate without HDR enhancer was not worse than with enhancer. In total, we have found two clones with a homozygous edit: one from the batch without HDR enhancer and one treated with $20 \mu \mathrm{M}$ enhancer. Heterozygous edits were expectedly more frequent, with four clones from the preparation without HDR enhancer and three clones with $20 \mu \mathrm{M}$ and $30 \mu \mathrm{M}$ of HDR enhancer, respectively. So far, the predictions from the amplicon sequencing deviated greatly from our final number of clones. A reason could be that our sample number per batch (16) was too low to allow precise statistical 
predictions. Overall, amplicon sequencing can be considered a checkpoint test to see if the introduction of the CRISPRS/Cas9 complex occurred at all, and if the HDR was introduced into the genome.

The newly established iPSC lines are currently cultured, expanded, and monitored for stable introduction of the HDR template. Mixed clones are separated and tested with Sanger sequencing. As a next step, edited iPSC lines are going to be differentiated into iPSC-CMs and tested for susceptibility to DOX treatment.

\subsection{Telomeres in cardiac diseases}

Recently discovered connections between telomere biology and cardiac diseases raised the question if telomere length is also altered in our human iPSC-CM model of ACT and if increased telomerase activity could rescue the ACT phenotype. In order to investigate this possibility, we first measured telomere length in iPSCs and iPSC-CMs from ACT and control patients. We found that telomeres were longer in iPSCs than in IPSC-CMs in both the ACT patient and control patient groups (Figure 5-14). Furthermore, we showed that the relative length of telomeres was shorter in ACT-iPSC-CMs compared to control patients (Figure 5-15). These data are in line with studies showing a clear link between cardiac diseases and telomere length (reviewed by Martínez and Blasco, 2018). Recently, a study found that telomere length in leukocytes correlates with the severity of the cardiomyopathy (Chatterjee et al., 2018). Furthermore, telomere-related SNPs were associated with ventricular arrhythmias in ischemic cardiomyopathy, which suggests that telomere length and telomere SNPs could be used as biomarkers to predict cardiac disease predisposition, development, and severity (Sawhney et al., 2016, 2018; Chang and Blau, 2018).

In ACT, a significant part of the detrimental effects of DOX is attributed to increased ROS production. Von Zglinicki and colleagues reported that oxidative stress shortens telomeres (von Zglinicki, 2002, 2006). Inversely, it was reported that high telomerase activity is connected to low mitochondrial $\mathrm{H}_{2} \mathrm{O}_{2}$ production (Beyer et al., 2016; reviewed by Quryshi et al., 2018), contradicting previous reports about ROS-induced mtDNA damage through mitochondrial hTERT (Santos et al., 2004, 2006). A role of TERT in mitochondria under oxidative stress is possible, because under oxidative stress, TERT is excluded from the nucleus and colocalizes with mitochondria (Haendeler et al., 2004, 2009). In this way, TERT protects human fibroblasts against oxidative stress (Ahmed et al., 2008). Compromised mitochondria have been linked to short telomeres (Chang et al., 2016) and telomerase deficiency predisposes mice to heart failure (Ait-Aissa et al., 2019). Another report showed an in vivo mouse model that telomerase expression protects the heart after acute MI (Bär et al., 2014). A tighter link between telomeric proteins and metabolic control is also possible: the telomeric protein TIN2 regulates mitochondrial oxidative phosphorylation (Chen et al., 
$2012 b)$. Considering that damaged mitochondria are a major source of ROS in ACT, our human iPSC-CM model of ACT is suitable to investigate this interaction in greater detail.

Initial experiments showed slightly longer telomeres in iPSC-CMs from control patients than from ACT patients. These results are going to be backed by a larger set of measurements. In the meantime, we have collected three samples per ACT patient and control patient of iPSCs and $\mathrm{PSSC}-\mathrm{CMs}$ to validate and confirm the initial results. Due to time constraints, it was not possible to include these results into this thesis. The samples are going to be measured by our collaborators Shambhabi Chatterjee and Christian Bär at the Medizinische Hochschule Hannover. That will allow us to estimate if the detected differences are merely aberrations one would expect from different cell lines, or if there is a consistent difference in telomere length between ACT patients and control patients. We performed a transcriptome analysis of ACT patient EHMs treated with $0.25 \mu \mathrm{M}$ DOX and compared them to an untreated patient group. We identified DEGs that are differentially expressed specifically in the ACT patient group after DOX treatment, but not in control EHMs. Interestingly, GO-term analysis of DOXinduced DEGs identified, among others, significantly increased expression of positive regulators of telomere maintenance in ACT-EHMs, but not in untreated controls.

The same holds for the comparisons between iPSCs and iPSC-CMs. These results show the expected tendency of longer telomeres in iPSCs compared to matched iPSC-CMs, which is in line with reports about telomere length in iPSCs (Huang et al., 2014). These initial experiments suggest that iPSC-CMs from ACT patients have shorter telomeres than iPSCCMs from control patients, which in turn could contribute to ACT-disease development. To test if longer telomeres and increased TERT expression can ameliorate DOX-induced detrimental effects on iPSC-CMs, we transduced our cells with AAV6-TERT vectors.

\subsection{Overexpression of TERT with AAV vectors}

Although telomere length in our iPSC-CMs has not been definitively determined, TERT overexpression could exert protective effects, because some of the beneficial effects of TERT activation were observed unrelated to telomere length (Ahmed et al., 2008). Protocols for AAV6-mediated TERT transduction in mice were established in the laboratory of Dr. Christian Bär at the Medizinische Hochschule Hannover and adapted to iPSC-CMs in our group. We used AAV6-GFP vectors to test transduction and determine the optimal $\mathrm{MOI}$ in our model. Due to limited vector and iPSC-CM availability, TERT expression in iPSC-CMs was not confirmed directly after transduction, which is a limitation. Nevertheless, expression levels of TERT in transduced iPSC-CMs were analyzed in Hannover and showed a 15-fold overexpression with an $\mathrm{MOI}$ of $1 \times 10^{4}$ (unpublished data, Shambhabi Chatterjee, personal communication). Positive GFP signals indicated that gene delivery with AAV6 vectors and gene expression worked as expected. Initially, iPSC-CMs were transduced with AAV6 with 
an MOI of $1 \times 10^{3}$ for $48 \mathrm{~h}$. Immediately after AAV6 transduction, iPSC-CMs were treated for three days with $0.25 \mu \mathrm{M}$ DOX and $1 \mu \mathrm{M}$ DOX. Subsequent apoptosis analysis showed that vector transduction itself induced apoptosis even in DOX-untreated iPSC-CMs (Figure 5-18). Apoptosis rates were 2.5- to 3.5-fold higher in AAV6-treated iPSC-CMs compared to AAV6untreated controls. DOX treatment increased apoptosis rates slightly more. To minimize the influence of AAV6 treatment on apoptosis rates, iPSC-CMs were given a recovery period of seven days before the start of DOX treatment (Figure 5-19D). Additionally, the DOX concentration was set to $0.5 \mu \mathrm{M}$. With these optimized treatment conditions, AAV6 transduced cells without DOX treatment had the same apoptosis rate of approximately $15 \%$ as untreated cells (Figure 5-19B). Unexpectedly, AAV6-TERT transduced iPSC-CMs did not show decreased apoptosis rates after DOX compared to empty AAV6-treated controls (Figure 5-19). Assessment of ROS production with an Amplex red assay also showed no effect on ROS production after AAV6 TERT transduction compared to the empty AAV6 control. The result was in line with the results from our apoptosis assay, but may be contrary to previous reports about reduced oxidative stress through TERT activation (reviewed by Quryshi et al., 2018). To validate our approach in the future, TERT expression and telomerase activation in iPSC-CMs after AAV6 need to be examined. All experiments were performed with an AAV6 vector containing a CMV promoter. To make the approach more specific for cardiac cells, AAV6 vectors with a cTnT promoter were produced and will be tested shortly in our ACT model. Besides, the $\mathrm{O}_{2}$ content of $20 \%$ in our incubators could be source of constant oxidative stress for iPSC-CMs. Reduction of the $\mathrm{O}_{2}$ content to $5 \%$ resulted in greater effect of DOX treatment on apoptosis rates (Dr. Christian Bär, personal communication).

Apoptosis and ROS production appear as results of a plethora of molecular interactions. To confidently assess the impact of TERT activation, it could be advantageous to choose a more direct readout of TERT performance. In the setting of DOX-treated iPSC-CMs, where we expect oxidative stress, mitochondrial function and/or mitochondrial ROS production would be a promising readout. Mitochondrial function can be assessed with a mito stress test with an Agilent Seahorse analyzer. The fluorescent probe MitoSOX is suitable for the microscopic study of mitochondrial ROS production. In this study, the MitoSOX assay was established for DOX-treated iPSC-CMs. Preliminary data show that the dynamic MitoSOX probe can detect DOX-induced increases of mitochondrial ROS in iPSC-CMs. In future experiments, the probe can be used to test compounds and treatment for their mitochondrial ROS-reducing properties in iPSC-CMs.

The suitability of TERT activation as a method to reduce side effects of cancer treatment is highly debated (Ling et al., 2012; Yan et al., 2015; Bär and Thum, 2017; Lipinska et al., 2017; Muñoz-Lorente et al., 2018). The cancer-driving characteristics of telomerase activity are well 
established. hTERT translocation from nuclei to mitochondria is increased in multidrugresistant hepatocellular carcinoma cells, which manifested in a protective effect for carcinoma cells (Ling et al., 2012; Yan et al., 2015). Reduced expression of telomerase in cancer cells increased their sensitivity to anticancer drugs such as DOX, cisplatin, and 5fluorouracil (reviewed by Lipinska et al., 2017). To rebut these concerns, Muñoz-Lorente et al. (2018) showed that AAV9-mediated telomerase activation did not increase the K-Rasdriven speed of lung cancer development in mice. In a viewpoint article, Bär and Thum (2017) provoke with the hypothesis that even in a cancer setting, the benefits of TERT activation would outperform its potential risks. In light of recent success rates of anti-cancer treatment and the increase in cancer survivors with severe long-term side effects, the potential of treatment options that ameliorate side effects should not be ostracized due to unfounded risk evaluations. Nevertheless, each case should be carefully assessed regarding relevant factors such as cancer type, classes of anti-cancer drugs, TERT expression of cancer cells, and the patient's predisposition to side effects.

\subsection{DCM cell size}

DCM can occur in a mixed form with HCM. Both diseases lead to similar symptoms that can include shortness of breath, leg swelling, and tiredness (Elliott, 2000; Japp et al., 2016). These symptoms stem from the heart's inability to pump blood efficiently. In DCM, stretched muscle fibers and a thinned ventricle wall of the left ventricle cause these symptoms. In $\mathrm{HCM}$, the walls of the heart become stiffer because they thicken. On a cellular level these two forms of cardiomyopathy can be distinguished by the size of cardiomyocytes (Brodehl et al., 2019).

Data sets in our lab from DCM patients with different genomic predisposition for the disease indicate that iPSC-CMs from DCM patients can differ greatly in size compared to healthy controls. Investigating the two DCM patients used in this study, we observed significantly smaller iPSC-CMs from DCM patient 1 compared to healthy controls and DCM patient 3 (Figure 5-25). iPSC-CMs of HCM lines usually show increased CM size (Eschenhagen and Carrier, 2019). DCM patient 1 presented with a severe case of sudden DCM. The extraordinarily small cell size could be due to a high level of sarcomeric disarray that could render the cell incapable of maintaining a larger scaffold (see section 6.8). iPSC-CMs from DCM patient 3 were significantly larger compared to iPSC-CMs from DCM patient 1 and healthy control (Figure 5-25). iPSC-CM size experiments were performed in 3D with a CASY cell counter. iPSC-CMs were measured after ten or eleven days on culture plates. Longer or shorter culture periods did have an effect on the measured cell sizes (data not shown). If the described $3 \mathrm{D}$ method or an alternative $2 \mathrm{D}$ approach mimics the situation in vivo best is up for debate. 


\subsection{Sarcomeric regularity}

Sarcomeres are crucial elements for the function of cardiomyocytes because they provide the structural backbone without which contraction of the cells, and therefore the main function of the entire organ, would not be feasible. Therefore, the morphological assessment of sarcomeric integrity can be used to estimate the functional impairment caused by the respective phenotype.

A previous dissertation from our laboratory has addressed the impact of DOX on the sarcomeric regularity of iPSC-CMs from the ACT cohort that was also used for this study (Haupt, 2018). iPSC-CMs from ACT patients are more impaired by DOX-induced sarcomeric irregularity compared to iPSC-CMs from control patients (Haupt, 2018). ACT and DCM share a similar phenotype and largely only differ in the trigger of the disease. Therefore, we were interested if effects of DOX on the sarcomere are universal in diseased iPSC-CMs, or if the susceptibility is specific to ACT-iPSC-CMs. Comparing iPSC-CMs from healthy individuals in this study to control patients from the ACT cohort, we found that sarcomeric decay after DOX treatment was comparable between the control groups with a slight dose-dependent decrease seen in both. We expected that DOX treatment would have a detrimental effect on the sarcomeres in CMs from healthy donors, because of earlier published studies, where it has been reported that DOX treatment leads to inhibition of myofilament gene transcription, and sarcomere disarray through inhibition of cardiac ankyrin repeat protein (CARP) transcription and subsequent depletion of CARP protein levels in rat cardiomyocytes (Chen et al., 2012a). Furthermore, it has been shown that DOX induces calpain-dependent titin proteolysis (Lim et al., 2004). One prominent effect of DOX is the elevation of cellular ROS levels. Under oxidative stress, ROS induces irreversible protein carbonylation (Levine et al., 1994). Carbonylation leads to depletion of crucial cellular proteins by tagging them for proteasomal degradation (Nyström, 2005). The amino acid residues lysine, arginine, proline, and threonine are particularly susceptible to carbonylation under oxidative stress (Amici et al., 1989; Stadtman, 1990). These four amino acids make up $25.6 \%$ of the cardiac myosin binding protein $\mathrm{C}$ (MYBPC), which has been described as selectively carbonylated during toxicity (Aryal et al., 2014). In our work, next generation sequencing data from 3D EHMs generated from ACT or control-iPSC-CMs showed that MYBPC3 was one of the genes that encodes for structural proteins that were down-regulated after DOX treatment. MYBPC3 is not only important for the function of striated muscle, but is also involved in its regulation, which makes it an interesting target for future studies (Gautel et al., 1995). Besides $M Y B P C 3$, our unpublished transcriptomic data show significant decreases in components of the actin cytoskeleton (18.03\%, e.g. ACTA2, MYBPC3, MYH6, MYH7, MYL2, MYL7, TNNI3, TNNT2), and muscle contraction (8.75\%, e.g. LDB3, MYBPC3, MYH6, MYL2, MYOM1, SORBS1, STMN1, TCAP, TNNT2) in ACT- and control EHMs after DOX treatment. These 
studies on protein depletion, together with our results on specific gene down-regulation and inhibition of global mRNA translation offer an explanation on how DOX treatment and resulting oxidative stress can lead to sarcomeric disarray.

An even more relevant question was to determine if we would see similar rates of sarcomeric degradation after DOX treatment in iPSC-CM from ACT patients and DCM patients. In our study, the decrease of sarcomeric regularity in iPSC-CMs from DCM patients was minimal with 0.05 -fold change after treatment with $1 \mu \mathrm{M}$ DOX (Figure 5-27B). In general, sarcomeric regularity in these DCM-iPSC-CMs was very low and deviated significantly from healthy iPSC-CMs. This is in line with frequent mutations in genes encoding for sarcomeric proteins in DCM patients (reviewed by Chang and Potter, 2005; Morimoto, 2008). Due to the very low values for sarcomeric regularity in the DCM group at baseline, the reduction following DOX treatment was not as strong as observed in cells from the ACT cohort. Considering the immunofluorescence images of the DCM-iPSC-CMs, it can be concluded that the patient iPSC-CMs from our DCM cohort are damaged to a higher degree at baseline than the ones from our ACT cohort. Increased heterogeneity in sarcomeric organization of IPSC-CMs of DCM patients has been reported previously (Sun et al., 2012). This conclusion is supported by the fact that the trigger for the DCM phenotype could be genetic in $30 \%$ of the cases and therefore inherent, while ACT only occurs after an external trigger, namely DOX treatment. Until now it has not been finally determined whether the used ACT- and DCM-patient's iPSCs have a genetic predisposition to the later disease. However, a decrease in sarcomeric regularity through DOX treatment and oxidative stress does not seem to be exclusive to ACT. Oxidative stress regulates proteolytic pathways, leading to a decrease of structural proteins, which has also been described in other disease settings, such as muscle atrophy in disuse atrophy (Powers et al., 2005). A recent review of multiple iPSC-CM studies on DCM concluded that sarcomere disorganization is a common finding in all disease lines (Eschenhagen and Carrier, 2019).

Taken together, the analysis of sarcomeric regularity after DOX treatment showed that disturbance of sarcomeric structures through DOX is a general effect of DOX treatment. Reduction of sarcomeric regularity in iPSC-CMs from Crt-DCM was comparable to the observed effect in iPSC-CMs of control patients of the ACT cohort. The very high amount of sarcomeric disarray in iPSC-CMs from DCM patients without DOX treatment makes it difficult to compare the treatment effect directly between ACT and DCM. 


\section{Limitations}

iPSC-CMs provide a consistent way of researching cellular processes in a human setting. This makes them a suitable tool to study human molecular mechanisms and is hence advantageous over animal models. However, disease modeling with iPSC-CMs also has limitations. A major limitation is the lack of maturity of iPSC-CMs. Even when cultured for at least 60 days, characteristics of iPSC-CMs resemble more the ones of CMs at a neonatal state rather than adult CMs (Kolanowski et al., 2017; Yoshida and Yamanaka, 2017). While metabolism and calcium handling do not differ much from adult CMs, the round, multiangeled morphology is distinct from the characteristic rod shape of mature CMs. For each research question, it has to be evaluated wheather the properties of iPSC-CMs are fitting to answer the particular research question, especially when investigating cardiac proteins that are differentially expressed throughout life such as Nox2 and Nox4. A shortcoming compared to animal studies or tissue samples is that iPSC-CM cultures represent monocultures of one cell type. That neglects interactions between different cell types and cell behavior in a natural organism. To reduce the bias, it is recommended to use organoids that mimic the nature of an organ better (Hudson et al., 2019). Besides, iPSC-CMs can be used in a wide range of biological assays. The ability to define cell numbers, add treatments, and control the culture conditions has benefits over tissue samples. However, the development of iPSC lines and the production of iPSC-CMs are cost and labor intensive. That sets boundaries to the sample numbers, which in turn hamperes genomic studies with iPSC cohorts. Furthermore, the small sample number combined with natural occurring inter-individual genetic variability manifests a hurdle to data interpretation. To notice abberrations that stem for a patient's genetic background, the aim was to use at least 2 different iPSC clones per person. An additional measure is the use of appropriate controls that match gender, age, disease history, etc. of the patients. In this study, there was a female patient (ACT patient 3) that did not have a matched female control (see section 3.1). In future studies it would be best to use isogenic controls that were created with gene editing (Figure 2-2).

Overall, iPSC-CMs are a valuable tool to research human disease mechanisms on a cellular level. Being aware of and managing the limitations of this model secured consistent and reliable data. 


\section{Conclusion and outlook}

This study addressed a broad range of ACT-related topics, from subcellular drug distribution over risk factor validation to emerging therapies to overcome ACT in the clinic. In this way, it displays the versatile options the iPSC model offers to investigate cardiac diseases. Especially in combination with CRISPR/Cas9, human IPSC models benefit the research on disease-associated mutations, risk assessment, and drug screening.

While we described for the first time intracellular levels of DOX and distribution thereof in iPSC-CMs, we also presented a protocol for measurement of intracellular DOX content with HPLC. This protocol could be adjusted to work with other cellular samples and tissues, and hence be applied in future molecular or clinical studies of ACT.

Currently, we are working on characterizing and validating the CRISPR/Cas9-edited iPSC lines. Moving forward, tests with iPSC-CMs from these isogenic iPSC lines on susceptibility towards DOX-induced sarcomeric damage, ROS production, and changes in calcium handling will reveal the impact of the described SNP on disease development. In this way, other ACT-associated SNPs can be tested for their relevance and the molecular mechanisms behind the clinical correlation can be revealed.

iPSC-CMs offer a platform to screen cardioprotective drugs like ranolazine or dexrazoxane in a human in vitro setting. The possibility to try different ratios of DOX and adjuvant drug makes it very versatile and the use of human patient-specific iPSC-CMs gives high relevance to the approach.

In the following months, we, together with our collaborators, will measure telomere length in a large set of generated iPSCs and iPSC-CMs from ACT and DCM patients. This will be the basis for assessments of telomere status in our models and potential involvements of telomere dysregulation in the specific patient disease history. Furthermore, we will continue to optimize in vitro TERT overexpression in iPSC-CMs to evaluate its utility as a potential treatment for ACT or cardiomyopathies in general.

A remaining question is in which way and through which pathways DOX exposure influences global mRNA translation. To better understand ROS production and ROS signaling in ACT, it is promising to investigate the crosstalk between Nox2 and mitochondria, both of which are key ROS producers. Gaining a deeper understanding of the exact interactions on the molecular level is crucial for specific interventions on ACT.

In cardiac research, disease modelling with human iPSCs is a valuable tool to discover molecular mechanisms, validate results from animal experiments and confirm data from clinical studies in a highly controllable in vitro model. 


\section{References}

Abraham, S.A., Waterhouse, D.N., Mayer, L.D., Cullis, P.R., Madden, T.D., and Bally, M.B. (2005). The liposomal formulation of doxorubicin. Methods Enzymol. 391, 71-97.

Adachi, K., Fujiura, Y., Mayumi, F., Nozuhara, A., Sugiu, Y., Sakanashi, T., Hidaka, T., and Toshima, H. (1993). A deletion of mitochondrial DNA in murine doxorubicin-induced cardiotoxicity. Biochem. Biophys. Res. Commun. 195, 945-951.

Agudelo, D., Bourassa, P., Bérubé, G., and Tajmir-Riahi, H.A. (2014). Intercalation of antitumor drug doxorubicin and its analogue by DNA duplex: Structural features and biological implications. Int. J. Biol. Macromol. 66, 144-150.

Ahmed, S., Passos, J.F., Birket, M.J., Beckmann, T., Brings, S., Peters, H., Birch-Machin, M.A., von Zglinicki, T., and Saretzki, G. (2008). Telomerase does not counteract telomere shortening but protects mitochondrial function under oxidative stress. J. Cell Sci. 121, 1046-1053.

Ait-Aissa, K., Heisner, J.S., Norwood Toro, L.E., Bruemmer, D., Doyon, G., Harmann, L., Geurts, A., Camara, A.K.S., and Beyer, A.M. (2019). Telomerase deficiency predisposes to heart failure and ischemia-reperfusion injury. Front. Cardiovasc. Med. 6, 31.

Alves, A.C., Magarkar, A., Horta, M., Lima, J.L.F.C., Bunker, A., Nunes, C., and Reis, S. (2017). Influence of doxorubicin on model cell membrane properties: Insights from in vitro and in silico studies. Sci.

Rep. 7, 6343.

Ambasta, R.K., Kumar, P., Griendling, K.K., Schmidt, H.H.H.W., Busse, R., and Brandes, R.P. (2004). Direct interaction of the novel Nox proteins with p22phox is required for the formation of a functionally active NADPH oxidase. J. Biol. Chem. 279, 45935-45941.

Amici, A., Levine, R.L., Tsai, L., and Stadtman, E.R. (1989). Conversion of amino acid residues in proteins and amino acid homopolymers to carbonyl derivatives by metal-catalyzed oxidation reactions. J. Biol. Chem. 264, 3341-3346.

Angsutararux, P., Luanpitpong, S., and Issaragrisil, S. (2015). Chemotherapy-induced cardiotoxicity: Overview of the roles of oxidative stress. Oxid. Med. Cell. Longev. 2015, 795602.

Arcamone, F., Cassinelli, G., Fantini, G., Grein, A., Orezzi, P., Pol, C., and Spalla, C. (1969). Adriamycin, 14-hydroxydaimomycin, a new antitumor antibiotic from $S$. Peucetius var.caesius. Biotechnol. Bioeng. 11, 1101-1110.

Aryal, B., and Rao, V.A. (2016). Deficiency in cardiolipin reduces doxorubicin-induced oxidative stress and mitochondrial damage in human B-lymphocytes. PLoS One 11, e0158376.

Aryal, B., Jeong, J., and Rao, V.A. (2014). Doxorubicin-induced carbonylation and degradation of cardiac myosin binding protein C promote cardiotoxicity. Proc. Natl. Acad. Sci. U. S. A. 111, 20112016.

Azarova, A.M., Lyu, Y.L., Lin, C.P., Tsai, Y.C., Lau, J.Y.N., Wang, J.C., and Liu, L.F. (2007). Roles of DNA topoisomerase II isozymes in chemotherapy and secondary malignancies. Proc. Natl. Acad. Sci. U. S. A. 104, 11014-11019.

Ball, G., Demmerle, J., Kaufmann, R., Davis, I., Dobbie, I.M., and Schermelleh, L. (2015). SIMcheck: A toolbox for successful super-resolution structured illumination microscopy. Sci. Rep. 5, 15915.

Bär, C., and Thum, T. (2017). Changing direction. From therapeutic telomerase inhibition to activation? Circ. Res. 120, 1393-1395.

Bär, C., De Jesus, B.B., Serrano, R., Tejera, A., Ayuso, E., Jimenez, V., Formentini, I., Bobadilla, M., Mizrahi, J., de Martino, A., et al. (2014). Telomerase expression confers cardioprotection in the adult 
mouse heart after acute myocardial infarction. Nat. Commun. 5, 5863.

Barbosa, R.R., Bourguignon, T.B., Torres, L.D., Arruda, L.S., Jacques, T. de M., Serpa, R.G., Calil, O. de A., and Barbosa, L.F.M. (2018). Anthracycline-associated cardiotoxicity in adults: Systematic review on the cardioprotective role of beta-blockers. Rev. Assoc. Med. Bras. 64, 745-754.

Bartlett, J.J., Trivedi, P.C., and Pulinilkunnil, T. (2017). Autophagic dysregulation in doxorubicin cardiomyopathy. J. Mol. Cell. Cardiol. 104, 1-8.

Bedard, K., and Krause, K.H. (2007). The NOX family of ROS-generating NADPH oxidases: physiology and pathophysiology. Physiol. Rev. 87, 245-313.

El Benna, J., Faust, R.P., Johnson, J.L., and Babior, B.M. (1996). Phosphorylation of the respiratory burst oxidase subunit $\mathrm{p} 47$ phox as determined by two-dimensional phosphopeptide mapping. Phosphorylation by protein kinase $\mathrm{C}$, protein kinase $\mathrm{A}$, and a mitogen-activated protein kinase. J. Biol. Chem. 271, 6374-6378.

Berthiaume, J.M., and Wallace, K.B. (2007). Adriamycin-induced oxidative mitochondrial cardiotoxicity. Cell Biol. Toxicol. 23, 15-25.

Beyer, A.M., Freed, J.K., Durand, M.J., Riedel, M., Ait-Aissa, K., Green, P., Hockenberry, J.C., Garret Morgan, R., Donato, A.J., Peleg, R., et al. (2016). Critical role for telomerase in the mechanism of flow-mediated dilation in the human microcirculation. Circ. Res. 118, 856-866.

Blanco, J.G., Sun, C.L., Landier, W., Chen, L., Esparza-Duran, D., Leisenring, W., Mays, A., Friedman, D.L., Ginsberg, J.P., Hudson, M.M., et al. (2012). Anthracycline-related cardiomyopathy after childhood cancer: Role of polymorphisms in carbonyl reductase genes - A report from the Children's Oncology Group. J. Clin. Oncol. 30, 1415-1421.

Blasco, M.A. (2005). Telomeres and human disease: ageing, cancer and beyond. Nat. Rev. Genet. 6, 611-622.

Bodnar, A.G., Ouellette, M., Frolkis, M., Holt, S.E., Chiu, C.P., Morin, G.B., Harley, C.B., Shay, J.W., Lichtsteiner, S., and Wright, W.E. (1998). Extension of life-span by introduction of telomerase into normal human cells. Science 279, 349-352.

Bokoch, G.M., and Diebold, B.A. (2002). Current molecular models for NADPH oxidase regulation by Rac GTPase. Blood 100, 2692-2696.

Bokoch, G.M., Diebold, B., Kim, J.S., and Gianni, D. (2009). Emerging evidence for the importance of phosphorylation in the regulation of NADPH oxidases. Antioxidants Redox Signal. 11, 2429-2441.

Bolotin, A., Quinquis, B., Sorokin, A., and Dusko Ehrlich, S. (2005). Clustered regularly interspaced short palindrome repeats (CRISPRs) have spacers of extrachromosomal origin. Microbiology 151, 2551-2561.

Borchert, T., Hübscher, D., Guessoum, C.I., Lam, T.-D.D., Ghadri, J.R., Schellinger, I.N., Tiburcy, M., Liaw, N.Y., Li, Y., Haas, J., et al. (2017). Catecholamine-dependent $\beta$-adrenergic signaling in a pluripotent stem cell model of Takotsubo cardiomyopathy. J. Am. Coll. Cardiol. 70, 975-991.

Brandes, R.P., Weissmann, N., and Schröder, K. (2014a). Nox family NADPH oxidases: Molecular mechanisms of activation. Free Radic. Biol. Med. 76, 208-226.

Brandes, R.P., Weissmann, N., and Schröder, K. (2014b). Redox-mediated signal transduction by cardiovascular Nox NADPH oxidases. J. Mol. Cell. Cardiol. 73, 70-79.

Brieger, K., Schiavone, S., Miller, F.J., and Krause, K.H. (2012). Reactive oxygen species: From health to disease. Swiss Med. Wkly. 142, w13659.

Brodehl, A., Ebbinghaus, H., Deutsch, M.-A., Gummert, J., Gärtner, A., Ratnavadivel, S., and Milting, 
H. (2019). Human induced pluripotent stem-cell-derived cardiomyocytes as models for genetic cardiomyopathies. Int. J. Mol. Sci. 20, 4381.

Burridge, P.W., Matsa, E., Shukla, P., Lin, Z.C., Churko, J.M., Ebert, A.D., Lan, F., Diecke, S., Huber, B., Mordwinkin, N.M., et al. (2014). Chemically defined generation of human cardiomyocytes. Nat. Methods 11, 855-860.

Burridge, P.W., Li, Y.F., Matsa, E., Wu, H., Ong, S.-G., Sharma, A., Holmström, A., Chang, A.C., Coronado, M.J., Ebert, A.D., et al. (2016). Human induced pluripotent stem cell-derived cardiomyocytes recapitulate the predilection of breast cancer patients to doxorubicin-induced cardiotoxicity. Nat. Med. 22, 547-556.

Cabrera Cabrera, J.R. (1999). Idiopathic dilated cardiomyopathy. Med. Hypotheses 53, 260-262.

Cai, F., Luis, M., Lin, X., Wang, M., Cai, L., Cen, C., and Biskup, E. (2019). Anthracycline-induced cardiotoxicity in the chemotherapy treatment of breast cancer: Preventive strategies and treatment. Mol. Clin. Oncol. 11, 15-23.

Camerino, B., and Palamidessi, G. (1960). Derivati della parazina II. Sulfonamdopir. Gazz Chim Ital 90, 1802-1815.

Canela, A., Vera, E., Klatt, P., and Blasco, M.A. (2007). High-throughput telomere length quantification by FISH and its application to human population studies. Proc. Natl. Acad. Sci. U. S. A. 104, 5300-5305.

Cao, Y., Shen, T., Huang, X., Lin, Y., Chen, B., Pang, J., Li, G., Wang, Q., Zohrabian, S., Duan, C., et al. (2017). Astragalus polysaccharide restores autophagic flux and improves cardiomyocyte function in doxorubicin-induced cardiotoxicity. Oncotarget 8, 4837-4848.

Cappetta, D., De Angelis, A., Sapio, L., Prezioso, L., Illiano, M., Quaini, F., Rossi, F., Berrino, L., Naviglio, S., and Urbanek, K. (2017). Oxidative stress and cellular response to doxorubicin: A common factor in the complex milieu of anthracycline cardiotoxicity. Oxid. Med. Cell. Longev. 2017.

Cardinale, D., Colombo, A., Bacchiani, G., Tedeschi, I., Meroni, C.A., Veglia, F., Civelli, M., Lamantia, G., Colombo, N., Curigliano, G., et al. (2015). Early detection of anthracycline cardiotoxicity and improvement with heart failure therapy. Circulation 131, 1981-1988.

Cave, A.C., Brewer, A.C., Narayanapanicker, A., Ray, R., Grieve, D.J., Walker, S., and Shah, A.M. (2006). NADPH oxidases in cardiovascular health and disease. Antioxidants Redox Signal. 8, 691-728.

Cawthon, R.M. (2002). Telomere measurement by quantitative PCR. Nucleic Acids Res. 30, 47e - 47.

Chang, A.C.Y., and Blau, H.M. (2018). Short telomeres - A hallmark of heritable cardiomyopathies. Differentiation 100, 31-36.

Chang, A.N., and Potter, J.D. (2005). Sarcomeric protein mutations in dilated cardiomyopathy. Heart Fail. Rev. 10, 225-235.

Chang, A.C.Y., Ong, S.-G., LaGory, E.L., Kraft, P.E., Giaccia, A.J., Wu, J.C., and Blau, H.M. (2016). Telomere shortening and metabolic compromise underlie dystrophic cardiomyopathy. Proc. Natl. Acad. Sci. 113, 13120-13125.

Chatterjee, S., de Gonzalo-Calvo, D., Derda, A.A., Schimmel, K., Sonnenschein, K., Bavendiek, U., Bauersachs, J., Bär, C., and Thum, T. (2018). Leukocyte telomere length correlates with hypertrophic cardiomyopathy severity. Sci. Rep. 8, 11227.

Chen, B., Zhong, L., Roush, S.F., Pentassuglia, L., Peng, X., Samaras, S., Davidson, J.M., Sawyer, D.B., and Lim, C.C. (2012a). Disruption of a GATA4/Ankrd1 signaling axis in cardiomyocytes leads to sarcomere disarray: Implications for anthracycline cardiomyopathy. PLoS One 7, e35743. 
Chen, L.-Y., Zhang, Y., Zhang, Q., Li, H., Luo, Z., Fang, H., Kim, S.H., Qin, L., Yotnda, P., Xu, J., et al. (2012b). Mitochondrial localization of telomeric protein TIN2 links telomere regulation to metabolic control. Mol. Cell 47, 839-850.

Chen, W., Qiu, J., and Shen, Y. (2012c). Topoisomerase Il $\alpha$, rather than $I \beta$, is a promising target in development of anti-cancer drugs. Drug Discov. Ther. 6, 230-237.

Chihara, D., Westin, J.R., Oki, Y., Ahmed, M.A., Do, B., Fayad, L.E., Hagemeister, F.B., Romaguera, J.E., Fanale, M.A., Lee, H.J., et al. (2016). Management strategies and outcomes for very elderly patients with diffuse large B-cell lymphoma. Cancer 122, 3145-3151.

Chlanda, P., Mekhedov, E., Waters, H., Schwartz, C.L., Fischer, E.R., Ryham, R.J., Cohen, F.S., Blank, P.S., and Zimmerberg, J. (2016). The hemifusion structure induced by influenza virus haemagglutinin is determined by physical properties of the target membranes. Nat. Microbiol. 1, 16050.

Chung, W.B., and Youn, H.J. (2016). Pathophysiology and preventive strategies of anthracyclineinduced cardiotoxicity. Korean J. Intern. Med. 31, 625-633.

Concordet, J.-P., and Haeussler, M. (2018). CRISPOR: intuitive guide selection for CRISPR/Cas9 genome editing experiments and screens. Nucleic Acids Res. 46, W242-W245.

Cong, L., Ran, F.A., Cox, D., Lin, S., Barretto, R., Habib, N., Hsu, P.D., Wu, X., Jiang, W., Marraffini, L.A., et al. (2013). Multiplex genome engineering using CRISPR/Cas systems. Science 339, 819-823.

Cowgill, J.A., Francis, S.A., and Sawyer, D.B. (2019). Anthracycline and peripartum cardiomyopathies. Circ. Res. 124, 1633-1646.

Cui, N., Wu, F., Lu, W.J., Bai, R., Ke, B., Liu, T., Li, L., Lan, F., and Cui, M. (2019). Doxorubicin-induced cardiotoxicity is maturation dependent due to the shift from topoisomerase $\| \alpha$ to $\| \beta$ in human stem cell derived cardiomyocytes. J. Cell. Mol. Med. 23, 4627-4639.

van Dalen, E.C., Michiels, E.M., Caron, H.N., and Kremer, L.C. (2010). Different anthracycline derivates for reducing cardiotoxicity in cancer patients. Cochrane Database Syst. Rev. 2010, CD005006.

van Dalen, E.C., Raphaël, M.F., Caron, H.N., and Kremer, L.C. (2014). Treatment including anthracyclines versus treatment not including anthracyclines for childhood cancer. Cochrane Database Syst. Rev. CD006647.

Davies, K.J.A., and Doroshow, J.H. (1986). Redox cycling of anthracyclines by cardiac mitochondria. I. Anthracycline radical formation by NADH dehydrogenase. J. Biol. Chem. 261, 3060-3067.

Dec, G.W., and Fuster, V. (1994). Idiopathic dilated cardiomyopathy. N. Engl. J. Med. 331, 1564-1575.

Demant, E.J.F., and Jensen, P.K. (1983). Destruction of phosphilipids and respiratory-chain activity in pig-heart submitochondrial particles induced by an adriamycin-iron complex. Eur. J. Biochem. 132, 551-556.

Denel-Bobrowska, M., and Marczak, A. (2017). Structural modifications in the sugar moiety as a key to improving the anticancer effectiveness of doxorubicin. Life Sci. 178, 1-8.

Doroshow, J.H. (1983). Effect of anthracycline antibiotics on oxygen radical formation in rat heart. Cancer Res. 43, 460-472.

Doroshow, J.H., Locker, G.Y., and Myers, C.E. (1980). Enzymatic defenses of the mouse heart against reactive oxygen metabolites. J. Clin. Invest. 65, 128-135.

Ducrest, A.-L., Szutorisz, H., Lingner, J., and Nabholz, M. (2002). Regulation of the human telomerase reverse transcriptase gene. Oncogene 21, 541-552.

Dupuy, C., Ohayon, R., Valent, A., Noël-Hudson, M.S., Dème, D., and Virion, A. (1999). Purification of 
a novel flavoprotein involved in the thyroid NADPH oxidase. Cloning of the porcine and human cDNAs. J. Biol. Chem. 274, 37265-37269.

Dutta, D., Xu, J., Dirain, M.L.S., and Leeuwenburgh, C. (2014). Calorie restriction combined with resveratrol induces autophagy and protects 26 -month-old rat hearts from doxorubicin-induced toxicity. Free Radic. Biol. Med. 74, 252-262.

Dworakowski, R., Anilkumar, N., Zhang, M., and Shah, A.M. (2006). Redox signalling involving NADPH oxidase-derived reactive oxygen species. Biochem. Soc. Trans. 34, 960-964.

El-Kareh, A.W., and Secomb, T.W. (2000). A mathematical model for comparison of bolus injection, continuous infusion, and liposomal delivery of doxorubicin to tumor cells. Neoplasia 2, 325-338.

Elens, L., Tyteca, D., Panin, N., Courtoy, P., Lison, D., Demoulin, J.B., and Haufroid, V. (2011). Functional defect caused by the 4544G>A SNP in ABCC2: Potential impact for drug cellular disposition. Pharmacogenet. Genomics 21, 884-893.

Elliott, P. (2000). Diagnosis and management of dilated cardiomyopathy. Heart 84, 106-112.

Eschenhagen, T., and Carrier, L. (2019). Cardiomyopathy phenotypes in human-induced pluripotent stem cell-derived cardiomyocytes-a systematic review. Pflugers Arch. Eur. J. Physiol. 471, 755-768.

Eschenhagen, T., Mummery, C., and Knollmann, B.C. (2015). Modelling sarcomeric cardiomyopathies in the dish: from human heart samples to iPSC cardiomyocytes. Cardiovasc. Res. 105, 424-438.

Escribá, P. V., Sastre, M., and García-Sevilla, J.A. (1995). Disruption of cellular signaling pathways by daunomycin through destabilization of nonlamellar membrane structures. Proc. Natl. Acad. Sci. U. S. A. $92,7595-7599$.

Ewer, M.S., and Ewer, S.M. (2015). Cardiotoxicity of anticancer treatments. Nat. Rev. Cardiol. 12, 547-558.

Fatkin, D., Yeoh, T., Hayward, C.S., Benson, V., Sheu, A., Richmond, Z., Feneley, M.P., Keogh, A.M., and Macdonald, P.S. (2011). Evaluation of left ventricular enlargement as a marker of early disease in familial dilated cardiomyopathy. Circ. Cardiovasc. Genet. 4, 342-348.

Fernandes, D.C., Wosniak, J., Pescatore, L. a, Bertoline, M. a, Liberman, M., Laurindo, F.R.M., and Santos, C.X.C. (2007). Analysis of DHE-derived oxidation products by HPLC in the assessment of superoxide production and NADPH oxidase activity in vascular systems. Am. J. Physiol. Cell Physiol. 292, C413-C422.

Flores, I., Cayuela, M.L., and Blasco, M.A. (2005). Effects of telomerase and telomere length on epidermal stem cell behavior. Science 309, 1253-1256.

Frit, P., Barboule, N., Yuan, Y., Gomez, D., and Calsou, P. (2014). Alternative end-joining pathway(s): Bricolage at DNA breaks. DNA Repair (Amst). 17, 81-97.

Galluzzi, L., Baehrecke, E.H., Ballabio, A., Boya, P., Bravo-San Pedro, J.M., Cecconi, F., Choi, A.M., Chu, C.T., Codogno, P., Colombo, M.I., et al. (2017). Molecular definitions of autophagy and related processes. EMBO J. 36, 1811-1836.

Gasiunas, G., Barrangou, R., Horvath, P., and Siksnys, V. (2012). Cas9-crRNA ribonucleoprotein complex mediates specific DNA cleavage for adaptive immunity in bacteria. Proc. Natl. Acad. Sci. 109, E2579-E2586.

Gautel, M., Zuffardi, O., Freiburg, A., and Labeit, S. (1995). Phosphorylation switches specific for the cardiac isoform of myosin binding protein-C: a modulator of cardiac contraction? EMBO J. 14, 19521960.

Gerull, B., Gramlich, M., Atherton, J., McNabb, M., Trombitás, K., Sasse-Klaassen, S., Seidman, J.G., 
Seidman, C., Granzier, H., Labeit, S., et al. (2002). Mutations of TTN, encoding the giant muscle filament titin, cause familial dilated cardiomyopathy. Nat. Genet. 30, 201-204.

Ghigo, A., Li, M., and Hirsch, E. (2016). New signal transduction paradigms in anthracycline-induced cardiotoxicity. Biochim. Biophys. Acta - Mol. Cell Res. 1863, 1916-1925.

Gilleron, M., Marechal, X., Montaigne, D., Franczak, J., Neviere, R., and Lancel, S. (2009). NADPH oxidases participate to doxorubicin-induced cardiac myocyte apoptosis. Biochem. Biophys. Res. Commun. 388, 727-731.

Giordano, S.H., Lin, Y.L., Kuo, Y.F., Hortobagyi, G.N., and Goodwin, J.S. (2012). Decline in the use of anthracyclines for breast cancer. J. Clin. Oncol. 30, 2232-2239.

Gonzalez, R., Lee, J.W., and Schultz, P.G. (2011). Stepwise chemically induced cardiomyocyte specification of human embryonic stem cells. Angew. Chemie - Int. Ed. 50, 11181-11185.

Goormaghtigh, E., and Ruysschaert, J.M. (1984). Anthracycline glycoside-membrane interactions. BBA - Rev. Biomembr. 779, 271-288.

Goormaghtigh, E., Chatelain, P., Caspers, J., and Ruysschaert, J.M. (1980). Evidence of a specific complex between adriamycin and negatively-charged phospholipids. BBA - Biomembr. 597, 1-14.

Goormaghtigh, E., Huart, P., Brasseur, R., and Ruysschaert, J.M. (1990). Structure of the adriamycincardiolipin complex. Arch. Int. Physiol. Biochim. 94, 247-257.

Gosalvez, M., Blanco, M., Hunter, J., Miko, M., and Chance, B. (1974). Effects of anticancer agents on the respiration of isolated mitochondria and tumor cells. Eur. J. Cancer 10, 567-574.

Greene, R.F., Jenkins, J.F., Speyer, J.L., Myers, C.E., and Collins, J.M. (1983). Plasma pharmacokinetics of adriamycin and adriamycinol: Implications for the design of in vitro experiments and treatment protocols. Cancer Res. 43, 3417-3421.

Greider, C.W., and Blackburn, E.H. (1985). Identification of a specific telomere terminal transferase activity in tetrahymena extracts. Cell 43, 405-413.

Groarke, J.D., and Nohria, A. (2015). Editorial: Anthracycline cardiotoxicity a new paradigm for an old classic. Circulation 131, 1946-1949.

Groemping, Y., and Rittinger, K. (2005). Activation and assembly of the NADPH oxidase: A structural perspective. Biochem. J. 386, 401-416.

Groemping, Y., Lapouge, K., Smerdon, S.J., and Rittinger, K. (2003). Molecular basis of phosphorylation-induced activation of the NADPH oxidase. Cell 113, 343-355.

Guo, F., Sun, Y., Wang, X., Wang, H., Wang, J., Gong, T., Chen, X., Zhang, P., Su, L., Fu, G., et al. (2019). Patient-specific and gene-corrected induced pluripotent stem cell-derived cardiomyocytes elucidate single-cell phenotype of short QT syndrome. Circ. Res. 124, 66-78.

Gyöngyösi, M., Lukovic, D., Zlabinger, K., Spannbauer, A., Gugerell, A., Pavo, N., Traxler, D., Pils, D., Maurer, G., Jakab, A., et al. (2019). Liposomal doxorubicin attenuates cardiotoxicity via induction of interferon-related DNA damage resistance. Cardiovasc. Res.

Haase, A., Göhring, G., and Martin, U. (2017). Generation of non-transgenic iPS cells from human cord blood CD34+cells under animal component-free conditions. Stem Cell Res. 21, 71-73.

Haendeler, J., Hoffmann, J., Diehl, J.F., Vasa, M., Spyridopoulos, I., Zeiher, A.M., and Dimmeler, S. (2004). Antioxidants inhibit nuclear export of telomerase reverse transcriptase and delay replicative senescence of endothelial cells. Circ. Res. 94, 768-775.

Haendeler, J., Dröse, S., Büchner, N., Jakob, S., Altschmied, J., Goy, C., Spyridopoulos, I., Zeiher, A.M., 
Brandt, U., and Dimmeler, S. (2009). Mitochondrial telomerase reverse transcriptase binds to and protects mitochondrial DNA and function from damage. Arterioscler. Thromb. Vasc. Biol. 29, 929935.

Hafstad, A.D., Nabeebaccus, A.A., and Shah, A.M. (2013). Novel aspects of ROS signalling in heart failure. Basic Res. Cardiol. 108, 359.

Hanahan, D., and Weinberg, R.A. (2000). The hallmarks of cancer. Cell 100, 57-70.

Hancock, J.T., Desikan, R., and Neill, S.J. (2001). Role of reactive oxygen species in cell signalling pathways. Biochem. Soc. Trans. 29, 345.

Harrington, L., Zhou, W., McPhail, T., Oulton, R., Yeung, D.S.K., Mar, V., Bass, M.B., and Robinson, M.O. (1997). Human telomerase contains evolutionarily conserved catalytic and structural subunits. Genes Dev. 11, 3109-3115.

Hasinoff, B.B., Schnabl, K.L., Marusak, R.A., Patel, D., and Huebner, E. (2003). Dexrazoxane (ICRF-187) protects cardiac myocytes against doxorubicin by preventing damage to mitochondria. Cardiovasc. Toxicol. 3, 89-99.

Haupt, L. (2018). Modeling anthracycline-induced cardiotoxicity with patient-specific iPSCs. Doctoral thesis, University of Göttingen

Hayashi, H., Kobara, M., Abe, M., Tanaka, N., Gouda, E., Toba, H., Yamada, H., Tatsumi, T., Nakata, T., and Matsubara, H. (2008). Aldosterone nongenomically produces NADPH oxidase-dependent reactive oxygen species and induces myocyte apoptosis. Hypertens. Res. 31, 363-375.

Hayflick, L. (1965). The limited in vitro lifetime of human diploid cell strains. Exp. Cell Res. 37, 614636.

Hayflick, L., and Moorhead, P.S. (1961). The serial cultivation of human diploid cell strains. Exp. Cell Res. 25, 585-621.

Henriksen, P.A. (2018). Anthracycline cardiotoxicity: An update on mechanisms, monitoring and prevention. Heart 104, 971-977.

Herman, E.H., and Ferrans, V.J. (1997). Animal models of anthracycline cardiotoxicity: Basic mechanisms and cardioprotective activity. Prog. Pediatr. Cardiol. 8, 49-58.

Hervera, A., Santos, C.X., De Virgiliis, F., Shah, A.M., and Di Giovanni, S. (2019). Paracrine mechanisms of redox signalling for postmitotic cell and tissue regeneration. Trends Cell Biol. 29, 514-530.

Higgins, E.M., Bos, J.M., Dotzler, S.M., Kim, C.J., and Ackerman, M.J. (2019). MRAS variants cause cardiomyocyte hypertrophy in patient-specific iPSC-derived cardiomyocytes: Additional evidence for MRAS as a definitive Noonan syndrome-susceptibility gene. Circ. Genomic Precis. Med.

Hinson, J.T., Chopra, A., Nafissi, N., Polacheck, W.J., Benson, C.C., Swist, S., Gorham, J., Yang, L., Schafer, S., Sheng, C.C., et al. (2015). Titin mutations in iPS cells define sarcomere insufficiency as a cause of dilated cardiomyopathy. Science 349, 982-986.

Hixon, S.C., Ellis, C.N., and Daugherty, J.P. (1981). Heart mitochondrial DNA synthesis: Preferential inhibition by adriamycin. J. Mol. Cell. Cardiol. 13, 855-860.

Hockemeyer, D., and Jaenisch, R. (2016). Induced pluripotent stem cells meet genome editing. Cell Stem Cell 18, 573-586.

von Hoff, D.D., Layard, M.W., Basa, P., Davis, H.L., Von Hoff, A.L., Rozencweig, M., and Muggia, F.M. (1979). Risk factors for doxorubicin-induced congestive heart failure. Ann. Intern. Med. 91, 710-717.

Holmgren, G., Synnergren, J., Bogestål, Y., Améen, C., Åkesson, K., Holmgren, S., Lindahl, A., and 
Sartipy, P. (2015). Identification of novel biomarkers for doxorubicin-induced toxicity in human cardiomyocytes derived from pluripotent stem cells. Toxicology 328, 102-111.

Holmgren, G., Sartipy, P., Andersson, C.X., Lindahl, A., and Synnergren, J. (2018). Expression profiling of human pluripotent stem cell-derived cardiomyocytes exposed to doxorubicin-Integration and visualization of multi-omics data. Toxicol. Sci. 163, 182-195.

Holmström, K.M., and Finkel, T. (2014). Cellular mechanisms and physiological consequences of redox-dependent signalling. Nat. Rev. Mol. Cell Biol. 15, 411-421.

Hong, J., Okada, K., Kusano, T., Komazawa, Y., Kobayashi, M., Mizutani, A., Kamada, N., and Kuramoto, A. (1990). Reduced DNA topoisomerase II in VP-16 resistant mouse breast cancer cell line. Biomed. Pharmacother. 44, 41-45.

Hoshino, A., Mita, Y., Okawa, Y., Ariyoshi, M., Iwai-Kanai, E., Ueyama, T., Ikeda, K., Ogata, T., and Matoba, S. (2013). Cytosolic p53 inhibits parkin-mediated mitophagy and promotes mitochondrial dysfunction in the mouse heart. Nat. Commun. 4, 2308.

Hrdina, R., Geršl, V., Klimtová, I., Šimůnek, T., Macháčková, J., Adamcová, M., Rudolf, E., Peychl, J., and Červinka, M. (2000). Anthracycline-induced cardiotoxicity. Acta Medica Cordoba. 43, 75-82.

Huang, Y., Liang, P., Liu, D., Huang, J., and Songyang, Z. (2014). Telomere regulation in pluripotent stem cells. Protein Cell 5, 194-202.

Hudson, J.E., Raad, F., Tiburcy, M., Roa, A., Liao, M.-L.C., Muppala, V., Soong, P.L., Araki, T., Neel, B., Keller, G., et al. (2019). Directed self-organization of human and non-human primate heart muscle organoids from pluripotent stem cells. SSRN Electron. J. Under review.

Ichihara, S., Yamada, Y., Kawai, Y., Osawa, T., Furuhashi, K., Duan, Z., and Ichihara, G. (2007). Roles of oxidative stress and Akt signaling in doxorubicin cardiotoxicity. Biochem. Biophys. Res. Commun. 359, 27-33.

Ichikawa, Y., Ghanefar, M., Bayeva, M., Wu, R., Khechaduri, A., Naga Prasad, S. V., Mutharasan, R.K., Jairaj Naik, T., and Ardehali, H. (2014). Cardiotoxicity of doxorubicin is mediated through mitochondrial iron accumulation. J. Clin. Invest. 124, 617-630.

Ilic, D., and Ogilvie, C. (2017). Concise review: Human embryonic stem cells-what have we done? What are we doing? Where are we going? Stem Cells 35, 17-25.

Indran, I.R., Hande, M.P., and Pervaiz, S. (2011). hTERT overexpression alleviates intracellular ROS production, improves mitochondrial function, and inhibits ROS-mediated apoptosis in cancer cells. Cancer Res. 71, 266-276.

Jaenisch, R., and Young, R. (2008). Stem cells, the molecular circuitry of pluripotency and nuclear reprogramming. Cell 132, 567-582.

Jaffré, F., Miller, C.L., Schänzer, A., Evans, T., Roberts, A.E., Hahn, A., and Kontaridis, M.I. (2019). Inducible pluripotent stem cell-derived cardiomyocytes reveal aberrant extracellular regulated kinase 5 and mitogen-activated protein kinase kinase $1 / 2$ signaling concomitantly promote hypertrophic cardiomyopathy in RAF1 -associated Noonan syndrome. Circulation 140, 207-224.

Japp, A.G., Gulati, A., Cook, S.A., Cowie, M.R., and Prasad, S.K. (2016). The diagnosis and evaluation of dilated cardiomyopathy. J. Am. Coll. Cardiol. 67, 2996-3010.

Jinek, M., Chylinski, K., Fonfara, I., Hauer, M., Doudna, J.A., and Charpentier, E. (2012). A programmable dual-RNA-guided DNA endonuclease in adaptive bacterial immunity. Science 337, 816-821.

Johar, S., Cave, A.C., Narayanapanicker, A., Grieve, D.J., and Shah, A.M. (2006). Aldosterone mediates 
angiotensin II-induced interstitial cardiac fibrosis via a Nox2-containing NADPH oxidase. FASEB J. 20, 1546-1548.

Kaiserová, H., Šimunek, T., Štěrba, M., Den Hartog, G.J.M., Schröterová, L., Popelová, O., Geršl, V., Kvasničková, E., and Bast, A. (2007). New iron chelators in anthracycline-induced cardiotoxicity. Cardiovasc. Toxicol. 7, 145-150.

Kamisago, M., Sharma, S.D., DePalma, S.R., Solomon, S., Sharma, P., McDonough, B., Smoot, L., Mullen, M.P., Woolf, P.K., Wigle, E.D., et al. (2000). Mutations in sarcomere protein genes as a cause of dilated cardiomyopathy. N. Engl. J. Med. 343, 1688-1696.

Kanter, P.M., Bullard, G.A., Pilkiewicz, F.G., Mayer, L.D., Cullis, P.R., and Pavelic, Z.P. (1993). Preclinical toxicology study of liposome encapsulated doxorubicin (TLC D-99): Comparison with doxorubicin and empty liposomes in mice and dogs. In Vivo (Brooklyn). 7, 85-95.

Kaushik, D., and Bansal, G. (2015). Four new degradation products of doxorubicin: An application of forced degradation study and hyphenated chromatographic techniques. J. Pharm. Anal. 5, 285-295.

Kawaguchi, T., Takemura, G., Kanamori, H., Takeyama, T., Watanabe, T., Morishita, K., Ogino, A., Tsujimoto, A., Goto, K., Maruyama, R., et al. (2012). Prior starvation mitigates acute doxorubicin cardiotoxicity through restoration of autophagy in affected cardiomyocytes. Cardiovasc. Res. 96, 456-465.

Kawahara, T., Jackson, H.M., Smith, S.M.E., Simpson, P.D., and Lambeth, J.D. (2011). Nox5 forms a functional oligomer mediated by self-association of its dehydrogenase domain. Biochemistry 50 , 2013-2025.

Kellner, U., Sehested, M., Jensen, P.B., Gieseler, F., and Rudolph, P. (2002). Culprit and victim - DNA topoisomerase II. Lancet Oncol. 3, 235-243.

Khasnis, A., Jongnarangsin, K., Abela, G., Veerareddy, S., Reddy, V., and Thakur, R. (2005).

Tachycardia-induced cardiomyopathy : A review of literature. PACE - Pacing Clin. Electrophysiol. 28, 710-721.

Kilian, A., Bowtell, D.D.L., Abud, H.E., Hime, G.R., Venter, D.J., Keese, P.K., Duncan, E.L., Reddel, R.R., and Jefferson, R.A. (1997). Isolation of a candidate human telomerase catalytic subunit gene, which reveals complex splicing patterns in different cell types. Hum. Mol. Genet. 6, 2011-2019.

King, N.M., and Perrin, J. (2014). Ethical issues in stem cell research and therapy. Stem Cell Res. Ther. 5,85 .

Kitani, T., Ong, S.-G., Lam, C.K., Rhee, J.-W., Zhang, J.Z., Oikonomopoulos, A., Ma, N., Tian, L., Lee, J., Telli, M.L., et al. (2019). Human-induced pluripotent stem cell model of trastuzumab-induced cardiac dysfunction in patients with breast cancer. Circulation 139, 2451-2465.

Kneidl, B., Peller, M., Winter, G., Lindner, L.H., and Hossann, M. (2014). Thermosensitive liposomal drug delivery systems: state of the art review. Int. J. Nanomedicine 9, 4387-4398.

Kolanowski, T.J., Antos, C.L., and Guan, K. (2017). Making human cardiomyocytes up to date: Derivation, maturation state and perspectives. Int. J. Cardiol. 241, 379-386.

Koleini, N., and Kardami, E. (2017). Autophagy and mitophagy in the context of doxorubicin-induced cardiotoxicity. Oncotarget 8, 46663-46680.

Kosztelnik, M., Kurucz, A., Papp, D., Jones, E., Sigmond, T., Barna, J., Traka, M.H., Lorincz, T., Szarka, A., Banhegyi, G., et al. (2019). Suppression of AMPK/aak-2 by NRF2/SKN-1 down-regulates autophagy during prolonged oxidative stress. FASEB J. 33, 2372-2387.

Koyanagi-Aoi, M., Ohnuki, M., Takahashi, K., Okita, K., Noma, H., Sawamura, Y., Teramoto, I., Narita, 
M., Sato, Y., Ichisaka, T., et al. (2013). Differentiation-defective phenotypes revealed by large-scale analyses of human pluripotent stem cells. Proc. Natl. Acad. Sci. U. S. A. 110, 20569-20574.

Kümmerle, A., Krueger, T., Dusmet, M., Vallet, C., Pan, Y., Ris, H.B., and Decosterd, L.A. (2003). A validated assay for measuring doxorubicin in biological fluids and tissues in an isolated lung perfusion model: Matrix effect and heparin interference strongly influence doxorubicin measurements. J. Pharm. Biomed. Anal. 33, 475-494.

Laemmli, U.K. (1970). Cleavage of structural proteins during the assembly of the head of bacteriophage T4. Nature 227, 680-685.

Lamberti, M., Giovane, G., Garzillo, E.M., Avino, F., Feola, A., Porto, S., Tombolini, V., and Di Domenico, M. (2014). Animal models in studies of cardiotoxicity side effects from antiblastic drugs in patients and occupational exposed workers. Biomed Res. Int. 2014, 240642.

Laurindo, F.R.M., de Souza, H.P., de A. Pedro, M., and Janiszewski, M. (2002). Redox aspects if vascular response to Injury. Methods 352, 432-454.

Lebrecht, D., and Walker, U.A. (2007). Role of mtDNA lesions in anthracycline cardiotoxicity. Cardiovasc. Toxicol. 7, 108-113.

Lee, Y., Kwon, I., Jang, Y., Cosio-Lima, L., and Barrington, P. (2019). Endurance exercise attenuates doxorubicin-induced cardiotoxicity. Med. Sci. Sport. Exerc. 1.

Lefrak, E.A., Pitha, J., Rosenheim, S., and Gottlieb, J.A. (1973). A clinicopathologic analysis of adriamycin cardiotoxicity. Cancer 32, 302-314.

Legha, S.S., Benjamin, R.S., Mackay, B., Ewer, M., Wallace, S., Valdivieso, M., Rasmussen, S.L., Blumenschein, G.R., and Freireich, E.J. (1982). Reduction of doxorubicin cardiotoxicity by prolonged continuous intravenous infusion. Ann. Intern. Med. 96, 133-139.

Leong, S.L., Chaiyakunapruk, N., and Lee, S.W.H. (2017). Candidate gene association studies of anthracycline-induced cardiotoxicity: A systematic review and meta-analysis. Sci. Rep. 7, 39.

Leri, A., Malhotra, A., Liew, C.C., Kajstura, J., and Anversa, P. (2000). Telomerase activity in rat cardiac myocytes is age and gender dependent. J. Mol. Cell. Cardiol. 32, 385-390.

Levine, R.L., Williams, J.A., Stadtman, E.P., and Shacter, E. (1994). Carbonyl assays for determination of oxidatively modified proteins. Methods Enzymol. 233, 346-357.

Li, D.L., Wang, Z. V., Ding, G., Tan, W., Luo, X., Criollo, A., Xie, M., Jiang, N., May, H., Kyrychenko, V., et al. (2016). Doxorubicin blocks cardiomyocyte autophagic flux by inhibiting lysosome acidification. Circulation 133, 1668-1687.

Li, J., Wang, P.-Y., Long, N.A., Zhuang, J., Springer, D.A., Zou, J., Lin, Y., Bleck, C.K.E., Park, J.-H., Kang, J.-G., et al. (2019). p53 prevents doxorubicin cardiotoxicity independently of its prototypical tumor suppressor activities. Proc. Natl. Acad. Sci. U. S. A. 116, 19626-19634.

Lian, X., Zhang, J., Azarin, S.M., Zhu, K., Hazeltine, L.B., Bao, X., Hsiao, C., Kamp, T.J., and Palecek, S.P. (2013). Directed cardiomyocyte differentiation from human pluripotent stem cells by modulating Wnt/ $\beta$-catenin signaling under fully defined conditions. Nat. Protoc. 8, 162-175.

Lim, C.C., Zuppinger, C., Guo, X., Kuster, G.M., Helmes, M., Eppenberger, H.M., Suter, T.M., Liao, R., and Sawyer, D.B. (2004). Anthracyclines induce calpain-dependent titin proteolysis and necrosis in cardiomyocytes. J. Biol. Chem. 279, 8290-8299.

Lindsey, M.L., Lange, R.A., Parsons, H., Andrews, T., and Aune, G.J. (2014). The tell-tale heart: Molecular and cellular responses to childhood anthracycline exposure. Am. J. Physiol. - Hear. Circ. Physiol. 307, H1379-H1389. 
Ling, X., Wen, L., and Zhou, Y. (2012). Role of mitochondrial translocation of telomerase in hepatocellular carcinoma cells with multidrug resistance. Int. J. Med. Sci. 9, 545-554.

Lipinska, N., Romaniuk, A., Paszel-Jaworska, A., Toton, E., Kopczynski, P., and Rubis, B. (2017). Telomerase and drug resistance in cancer. Cell. Mol. Life Sci. 74, 4121-4132.

Lipshultz, S.E., Lipsitz, S.R., Mone, S.M., Goorin, A.M., Sallan, S.E., Sanders, S.P., Orav, E.J., Gelber, R.D., and Colan, S.D. (1995). Female sex and higher drug dose as risk factors for late cardiotoxic effects of doxorubicin therapy for childhood cancer. N. Engl. J. Med. 332, 1738-1744.

Liu, J.P. (1999). Studies of the molecular mechanisms in the regulation of telomerase activity. FASEB J. 13, 2091-2104.

Loos, B., Du Toit, A., and Hofmeyr, J.H.S. (2014). Defining and measuring autophagosome flux Concept and reality. Autophagy 10, 2087-2096.

López-Otín, C., Blasco, M.A., Partridge, L., Serrano, M., and Kroemer, G. (2013). The hallmarks of aging. Cell 153, 1194-1217.

Lotrionte, M., Biondi-Zoccai, G., Abbate, A., Lanzetta, G., D’Ascenzo, F., Malavasi, V., Peruzzi, M., Frati, G., and Palazzoni, G. (2013). Review and meta-analysis of incidence and clinical predictors of anthracycline cardiotoxicity. Am. J. Cardiol. 112, 1980-1984.

Lv, W., Qiao, L., Petrenko, N., Li, W., Owens, A.T., McDermott-Roe, C., and Musunuru, K. (2018). Functional annotation of TNNT2 variants of uncertain significance with genome-edited cardiomyocytes. Circulation 138, 2852-2854.

Lyon, P.C., Gray, M.D., Mannaris, C., Folkes, L.K., Stratford, M., Campo, L., Chung, D.Y.F., Scott, S., Anderson, M., Goldin, R., et al. (2018). Safety and feasibility of ultrasound-triggered targeted drug delivery of doxorubicin from thermosensitive liposomes in liver tumours (TARDOX): a single-centre, open-label, phase 1 trial. Lancet Oncol. 19, 1027-1039.

Lyu, Y.L., Kerrigan, J.E., Lin, C.P., Azarova, A.M., Tsai, Y.C., Ban, Y., and Liu, L.F. (2007). Topoisomerase $\| \beta$-mediated DNA double-strand breaks: Implications in doxorubicin cardiotoxicity and prevention by dexrazoxane. Cancer Res. 67, 8839-8846.

Ma, J., Wang, Y., Zheng, D., Wei, M., Xu, H., and Peng, T. (2013). Rac1 signalling mediates doxorubicin-induced cardiotoxicity through both reactive oxygen species-dependent and independent pathways. Cardiovasc. Res. 97, 77-87.

Magdy, T., Burmeister, B.T., and Burridge, P.W. (2016). Validating the pharmacogenomics of chemotherapy-induced cardiotoxicity: What is missing? Pharmacol. Ther. 168, 113-125.

Mahon, N.G., Murphy, R.T., MacRae, C.A., Caforio, A.L.P., Elliott, P.M., and McKenna, W.J. (2005). Echocardiographic evaluation in asymptomatic relatives of patients with dilated cardiomyopathy reveals preclinical disease. Ann. Intern. Med. 143.

Maillet, A., Tan, K., Chai, X., Sadananda, S.N., Mehta, A., Ooi, J., Hayden, M.R., Pouladi, M.A., Ghosh, S., Shim, W., et al. (2016). Modeling doxorubicin-induced cardiotoxicity in human pluripotent stem cell derived-cardiomyocytes. Sci. Rep. 6, 25333.

Manning, B.D., and Toker, A. (2017). AKT/PKB signaling: Navigating the network. Cell 169, 381-405.

Marciano, R., Leprivier, G., and Rotblat, B. (2018). Puromycin labeling does not allow protein synthesis to be measured in energy-starved cells correspondence. Cell Death Dis. 9, 39.

Martinez-Delgado, B., Yanowsky, K., Inglada-Perez, L., Domingo, S., Urioste, M., Osorio, A., and Benitez, J. (2011). Genetic anticipation is associated with telomere shortening in hereditary breast cancer. PLoS Genet. 7, e1002182. 
Martínez, P., and Blasco, M.A. (2018). Heart-breaking telomeres. Circ. Res. 123, 787-802.

Martyn, K.D., Frederick, L.M., Von Loehneysen, K., Dinauer, M.C., and Knaus, U.G. (2006). Functional analysis of Nox4 reveals unique characteristics compared to other NADPH oxidases. Cell. Signal. 18, $69-82$.

Matsa, E., Ahrens, J.H., and Wu, J.C. (2016). Human induced pluripotent stem cells as a platform for personalized and precision cardiovascular medicine. Physiol. Rev. 96, 1093-1126.

McGowan, J. V, Chung, R., Maulik, A., Piotrowska, I., Walker, J.M., and Yellon, D.M. (2017). Anthracycline chemotherapy and cardiotoxicity. Cardiovasc. Drugs Ther. 31, 63-75.

McMurray, J.J.V., Adamopoulos, S., Anker, S.D., Auricchio, A., Böhm, M., Dickstein, K., Falk, V., Filippatos, G., Fonseca, C., Gomez-Sanchez, M.A., et al. (2012). ESC Guidelines for the diagnosis and treatment of acute and chronic heart failure 2012. Eur. J. Heart Fail. 14, 803-869.

McMurray, J.J.V., Packer, M., Desai, A.S., Gong, J., Lefkowitz, M.P., Rizkala, A.R., Rouleau, J.L., Shi, V.C., Solomon, S.D., Swedberg, K., et al. (2014). Angiotensin-neprilysin inhibition versus enalapril in heart failure. N. Engl. J. Med. 371, 993-1004.

Meiners, B., Shenoy, C., and Zordoky, B.N. (2018). Clinical and preclinical evidence of sex-related differences in anthracycline-induced cardiotoxicity. Biol. Sex Differ. 9, 38.

Merkle, F.T., and Eggan, K. (2013). Modeling human disease with pluripotent stem cells: From genome association to function. Cell Stem Cell 12, 656-668.

Mertens, A.C., Liu, Q., Neglia, J.P., Wasilewski, K., Leisenring, W., Armstrong, G.T., Robison, L.L., and Yasui, Y. (2008). Cause-specific late mortality among 5 -year survivors of childhood cancer: The childhood cancer survivor study. J. Natl. Cancer Inst. 100, 1368-1379.

Meyerson, M., Counter, C.M., Eaton, E.N., Ellisen, L.W., Steiner, P., Caddle, S.D., Ziaugra, L., Beijersbergen, R.L., Davidoff, M.J., Qingyun, L., et al. (1997). hEST2, the putative human telomerase catalytic subunit gene, is up- regulated in tumor cells and during immortalization. Cell 90, 785-795.

Middleman, E., Luce, J., and Frei, E. (1971). Clinical trials with adriamycin. Cancer 28, 844-850.

Mikhail, A.S., Negussie, A.H., Pritchard, W.F., Haemmerich, D., Woods, D., Bakhutashvili, I., EsparzaTrujillo, J., Brancato, S.J., Karanian, J., Agarwal, P.K., et al. (2017). Lyso-thermosensitive liposomal doxorubicin for treatment of bladder cancer. Int. J. Hyperth. 33, 733-740.

Minotti, G., Menna, P., Salvatorelli, E., Cairo, G., and Gianni, L. (2004). Anthracyclines: molecular advances and pharmacologic developments in antitumor activity and cardiotoxicity. Pharmacol. Rev. $56,185-229$.

Mitra, M.S., Donthamsetty, S., White, B., Latendresse, J.R., and Mehendale, H.M. (2007). Mechanism of protection of moderately diet restricted rats against doxorubicin-induced acute cardiotoxicity. Toxicol. Appl. Pharmacol. 225, 90-101.

Moazeni, S., Cadeiras, M., Yang, E.H., Deng, M.C., and Nguyen, K.L. (2017). Anthracycline induced cardiotoxicity: biomarkers and "Omics" technology in the era of patient specific care. Clin. Transl. Med. 6, 17.

Mojica, F.J.M., Juez, G., and Rodriguez-Valera, F. (1993). Transcription at different salinities of Haloferax mediterranei sequences adjacent to partially modified Pstl sites. Mol. Microbiol. 9, 613621.

Mordente, A., Meucci, E., Martorana, G.E., Giardina, B., and Minotti, G. (2001). Human heart cytosolic reductases and anthracycline cardiotoxicity. In IUBMB Life, pp. 83-88.

Morimoto, S. (2008). Sarcomeric proteins and inherited cardiomyopathies. Cardiovasc. Res. 77, 659- 
666.

Motamarry, A., Asemani, D., and Haemmerich, D. (2017). Thermosensitive liposomes. In Liposomes, (InTech), pp. 187-211.

Motta, B.M., Pramstaller, P.P., Hicks, A.A., and Rossini, A. (2017). The impact of CRISPR/Cas9 technology on cardiac research: From disease modelling to therapeutic approaches. Stem Cells Int. 2017, 1-13.

Motulsky, H.J., and Brown, R.E. (2006). Detecting outliers when fitting data with nonlinear regression - A new method based on robust nonlinear regression and the false discovery rate. BMC Bioinformatics 7, 123.

Mross, K., Niemann, B., Massing, U., Drevs, J., Unger, C., Bhamra, R., and Swenson, C.E. (2004). Pharmacokinetics of liposomal doxorubicin (TLC-D99; Myocet) in patients with solid tumors: An open-label, single-dose study. Cancer Chemother. Pharmacol. 54, 514-524.

Mullis, K., Faloona, F., Scharf, S., Saiki, R., Horn, G., and Erlich, H. (1986). Specific enzymatic amplification of DNA in vitro: The polymerase chain reaction. Cold Spring Harb. Symp. Quant. Biol. $51,263-273$.

Muñoz-Lorente, M.A., Martínez, P., Tejera, Á., Whittemore, K., Moisés-Silva, A.C., Bosch, F., and Blasco, M.A. (2018). AAV9-mediated telomerase activation does not accelerate tumorigenesis in the context of oncogenic K-Ras-induced lung cancer. PLoS Genet. 14, e1007562.

Nabhan, C., Byrtek, M., Rai, A., Dawson, K., Zhou, X., Link, B.K., Friedberg, J.W., Zelenetz, A.D., Maurer, M.J., Cerhan, J.R., et al. (2015). Disease characteristics, treatment patterns, prognosis, outcomes and lymphoma-related mortality in elderly follicular lymphoma in the United States. Br. J. Haematol. 170, 85-95.

Nakamura, T.M., Morin, G.B., Chapman, K.B., Weinrich, S.L., Andrews, W.H., Lingner, J., Harley, C.B., and Cech, T.R. (1997). Telomerase catalytic subunit homologs from fission yeast and human. Science 277, 955-959.

Nicolay, K., Timmers, R.J.M., Spoelstra, E., Neut, R. Van Der, Fok, J.J., Huigen, Y.M., Verkleij, A.J., and Kruijff, B. De (1984). The interaction of adriamycin with cardiolipin in model and rat liver mitochondrial membranes. BBA - Biomembr. 778, 359-371.

Nozaki, N., Shishido, T., Takeishi, Y., and Kubota, I. (2004). Modulation of doxorubicin-induced cardiac dysfunction in toll-like receptor-2-knockout mice. Circulation 110, 2869-2874.

Nyström, T. (2005). Role of oxidative carbonylation in protein quality control and senescence. EMBO J. 24, 1311-1317.

Oh, H., and Schneider, M.D. (2002). The emerging role of telomerase in cardiac muscle cell growth and survival. J. Mol. Cell. Cardiol. 34, 717-724.

Ohtani, K., Fujino, T., Ide, T., Funakoshi, K., Sakamoto, I., Hiasa, K. ichi, Higo, T., Kamezaki, K., Akashi, K., and Tsutsui, H. (2019). Recovery from left ventricular dysfunction was associated with the early introduction of heart failure medical treatment in cancer patients with anthracycline-induced cardiotoxicity. Clin. Res. Cardiol. 108, 600-611.

Park, I.-H., Arora, N., Huo, H., Maherali, N., Ahfeldt, T., Shimamura, A., Lensch, M.W., Cowan, C., Hochedlinger, K., and Daley, G.Q. (2008). Disease-specific induced pluripotent stem cells. Cell 134, 877-886.

Park, J., Choi, S., Kim, H., Ji, S., Jang, W., Kim, J., Baek, S., and Kwon, S. (2016). Doxorubicin regulates autophagy signals via accumulation of cytosolic $\mathrm{Ca} 2+$ in human cardiac progenitor cells. Int. J. Mol. Sci. 17, 1680. 
Patnaik, J.L., Byers, T., DiGuiseppi, C., Dabelea, D., and Denberg, T.D. (2011). Cardiovascular disease competes with breast cancer as the leading cause of death for older females diagnosed with breast cancer: a retrospective cohort study. Breast Cancer Res. 13, R64.

Patten, I.S., Rana, S., Shahul, S., Rowe, G.C., Jang, C., Liu, L., Hacker, M.R., Rhee, J.S., Mitchell, J., Mahmood, F., et al. (2012). Cardiac angiogenic imbalance leads to peripartum cardiomyopathy. Nature 485, 333-338.

Pendyala, S., and Natarajan, V. (2010). Redox regulation of Nox proteins. Respir. Physiol. Neurobiol. $174,265-271$.

Petry, A., Djordjevic, T., Weitnauer, M., Kietzmann, T., Hess, J., and Görlach, A. (2006). NOX2 and NOX4 mediate proliferative response in endothelial cells. Antioxid. Redox Signal. 8, 1473-1484.

Pfreundschuh, M., Schubert, J., Ziepert, M., Schmits, R., Mohren, M., Lengfelder, E., Reiser, M., Nickenig, C., Clemens, M., Peter, N., et al. (2008). Six versus eight cycles of bi-weekly CHOP-14 with or without rituximab in elderly patients with aggressive CD20+ B-cell lymphomas: a randomised controlled trial (RICOVER-60). Lancet. Oncol. 9, 105-116.

Pioner, J.M., Guan, X., Klaiman, J.M., Racca, A.W., Pabon, L., Muskheli, V., Macadangdang, J., Ferrantini, C., Hoopmann, M.R., Moritz, R.L., et al. (2019). Absence of full-length dystrophin impairs normal maturation and contraction of cardiomyocytes derived from human-induced pluripotent stem cells. Cardiovasc. Res. (in print).

Plana, J.C., Galderisi, M., Barac, A., Ewer, M.S., Ky, B., Scherrer-Crosbie, M., Ganame, J., Sebag, I.A., Agler, D.A., Badano, L.P., et al. (2014). Expert consensus for multimodality imaging evaluation of adult patients during and after cancer therapy: A report from the American Society of Echocardiography and the European Association of Cardiovascular Imaging. Eur. Heart J. Cardiovasc. Imaging 15, 1063-1093.

Poklepovic, A., Qu, Y., Dickinson, M., Kontos, M.C., Kmieciak, M., Schultz, E., Bandopadhyay, D., Deng, X., and Kukreja, R.C. (2018). Randomized study of doxorubicin-based chemotherapy regimens, with and without sildenafil, with analysis of intermediate cardiac markers. Cardio-Oncology 4.

Poss, K.D., Wilson, L.G., and Keating, M.T. (2002). Heart regeneration in zebrafish. Science 298, 21882190.

Powers, S.K., Kavazis, A.N., and DeRuisseau, K.C. (2005). Mechanisms of disuse muscle atrophy: Role of oxidative stress. Am. J. Physiol. - Regul. Integr. Comp. Physiol. 288, R337-R344.

Qin, A., Thompson, C.L., and Silverman, P. (2015). Predictors of late-onset heart failure in breast cancer patients treated with doxorubicin. J. Cancer Surviv. 9, 252-259.

Quryshi, N., Norwood Toro, L.E., Ait-Aissa, K., Kong, A., and Beyer, A.M. (2018). Chemotherapeuticinduced cardiovascular dysfunction: Physiological effects, early detection-the role of telomerase to counteract mitochondrial defects and oxidative stress. Int. J. Mol. Sci. 19, 1-32.

Raad, H., Paclet, M.H., Boussetta, T., Kroviarski, Y., Morel, F., Quinn, M.T., Gougerot-Pocidalo, M.A., Dang, P.M.C., and El-Benna, J. (2009). Regulation of the phagocyte NADPH oxidase activity: Phosphorylation of gp91phox/NOX2 by protein kinase $C$ enhances its diaphorase activity and binding to Rac2, p67phox, and p47phox. FASEB J. 23, 1011-1022.

Racles, C., Zaltariov, M.F., Silion, M., Macsim, A.M., and Cozan, V. (2019). Photo-oxidative degradation of doxorubicin with siloxane MOFs by exposure to daylight. Environ. Sci. Pollut. Res. 26, 19684-19696.

Reho, J.J., Guo, D., and Rahmouni, K. (2019). Mechanistic target of rapamycin complex 1 signaling modulates vascular endothelial function through reactive oxygen species. J. Am. Heart Assoc. 8. 
Reichwagen, A., Ziepert, M., Kreuz, M., Gödtel-Armbrust, U., Rixecker, T., Poeschel, V., Reza Toliat, M., Nürnberg, P., Tzvetkov, M., Deng, S., et al. (2015). Association of NADPH oxidase polymorphisms with anthracycline-induced cardiotoxicity in the RICOVER-60 trial of patients with aggressive CD20(+) B-cell lymphoma. Pharmacogenomics 16, 361-372.

Ren, Y., Lee, M.Y., Schliffke, S., Paavola, J., Amos, P.J., Ge, X., Ye, M., Zhu, S., Senyei, G., Lum, L., et al. (2011). Small molecule Wnt inhibitors enhance the efficiency of BMP-4-directed cardiac differentiation of human pluripotent stem cells. J. Mol. Cell. Cardiol. 51, 280-287.

Riccardi, F., Mocerino, C., Barbato, C., Ambrosio, F., Festino, L., Vitale, M.G., Carrillo, G., Trunfio, M., Minelli, S., and Cartenì, G. (2013). First-line chemotherapy with liposomal doxorubicin plus cyclofosfamide in metastatic breast cancer: A case report of early and prolonged response. Int. J. Immunopathol. Pharmacol. 26, 773-778.

Richardson, G.D., Breault, D., Horrocks, G., Cormack, S., Hole, N., and Owens, W.A. (2012). Telomerase expression in the mammalian heart. FASEB J. 26, 4832-4840.

Robert, J. (2007). Preclinical assessment of anthracycline cardiotoxicity in laboratory animals: Predictiveness and pitfalls. In Cell Biology and Toxicology, pp. 27-37.

Rosenzweig, A. (2012). Cardiac regeneration. Science 338, 1549-1550.

Rossi, F., and Zatti, M. (1964). Biochemical aspects of phagocytosis in poly-morphonuclear leucocytes. NADH and NADPH oxidation by the granules of resting and phagocytizing cells. Experientia 20, 21-23.

Santos, C.X.C., Anilkumar, N., Zhang, M., Brewer, A.C., and Shah, A.M. (2011). Redox signaling in cardiac myocytes. Free Radic. Biol. Med. 50, 777-793.

Santos, J.H., Meyer, J.N., Skorvaga, M., Annab, L.A., and Van Houten, B. (2004). Mitochondrial hTERT exacerbates free-radical-mediated mtDNA damage. Aging Cell 3, 399-411.

Santos, J.H., Meyer, J.N., and Van Houten, B. (2006). Mitochondrial localization of telomerase as a determinant for hydrogen peroxide-induced mitochondrial DNA damage and apoptosis. Hum. Mol. Genet. 15, 1757-1768.

Sauter, K.A.D., Magun, E.A., lordanov, M.S., and Magun, B.E. (2010). ZAK is required for doxorubicin, a novel ribotoxic stressor, to induce SAPK activation and apoptosis in HaCaT cells. Cancer Biol. Ther. $10,258-266$.

Sawhney, V., Campbell, N.G., Brouilette, S.W., Coppen, S.R., Harbo, M., Baker, V., Ikebe, C., Shintani, Y., Hunter, R.J., Dhinoja, M., et al. (2016). Telomere shortening and telomerase activity in ischaemic cardiomyopathy patients - Potential markers of ventricular arrhythmia. Int. J. Cardiol. 207, 157-163.

Sawhney, V., Brouilette, S., Campbell, N., Coppen, S., Baker, V., Hunter, R., Dhinoja, M., Johnston, A., Earley, M., Sporton, S., et al. (2018). Association of genetic variation in telomere-related SNP and telomerase with ventricular arrhythmias in ischemic cardiomyopathy. PACE - Pacing Clin.

Electrophysiol. 41, 261-266.

Schirmer, M., Hoffmann, M., Kaya, E., Tzvetkov, M., and Brockmöller, J. (2008). Genetic polymorphisms of $\mathrm{NAD}(\mathrm{P}) \mathrm{H}$ oxidase: variation in subunit expression and enzyme activity. Pharmacogenomics J. 8, 297-304.

Schmidt, E.K., Clavarino, G., Ceppi, M., and Pierre, P. (2009). SUnSET, a nonradioactive method to monitor protein synthesis. Nat. Methods 6, 275-277.

Schubert, M., Thommandru, B., and Wang, B. (2018). IDT CRISPR HDR enhancer imporves HDR efficiency across multiple cell lines. 
Sciarretta, S., Yee, D., Ammann, P., Nagarajan, N., Volpe, M., Frati, G., and Sadoshima, J. (2015). Role of NADPH oxidase in the regulation of autophagy in cardiomyocytes. Clin. Sci. (Lond). 128, 387-403.

Sciarretta, S., Forte, M., Frati, G., and Sadoshima, J. (2018a). New insights into the role of mtor signaling in the cardiovascular system. Circ. Res. 122, 489-505.

Sciarretta, S., Maejima, Y., Zablocki, D., and Sadoshima, J. (2018b). The role of autophagy in the heart. Annu. Rev. Physiol. 80, 1-26.

Serrander, L., Cartier, L., Bedard, K., Banfi, B., Lardy, B., Plastre, O., Sienkiewicz, A., Fórró, L., Schlegel, W., and Krause, K.H. (2007). NOX4 activity is determined by mRNA levels and reveals a unique pattern of ROS generation. Biochem. J. 406, 105-114.

Serrano, J., Palmeira, C.M., Kuehl, D.W., and Wallace, K.B. (1999). Cardioselective and cumulative oxidation of mitochondrial DNA following subchronic doxorubicin administration. Biochim. Biophys. Acta - Bioenerg. 1411, 201-205.

Shah, P., Garris, R., Abboud, R., Vasudev, R., Patel, H., Doshi, R., Shamoon, F., and Bikkina, M. (2019). Meta-analysis comparing usefulness of beta blockers to preserve left ventricular function during anthracycline therapy. Am. J. Cardiol. 124, 789-794.

Sharpless, N.E., and DePinho, R.A. (2007). How stem cells age and why this makes us grow old. Nat. Rev. Mol. Cell Biol. 8, 703-713.

Sheppard, C.E., and Anwar, M. (2019). The use of sacubitril/valsartan in anthracycline-induced cardiomyopathy: A mini case series. J. Oncol. Pharm. Pract. 25, 1231-1234.

Shintani, T., and Klionsky, D.J. (2004). Autophagy in health and disease: A double-edged sword. Science 306, 990-995.

Silber, J.H., Jakacki, R.I., Larsen, R.L., Goldwein, J.W., and Barber, G. (1993). Increased risk of cardiac dysfunction after anthracyclines in girls. Med. Pediatr. Oncol. 21, 477-479.

Silva, A., Gírio, A., Cebola, I., Santos, C.I., Antunes, F., and Barata, J.T. (2011). Intracellular reactive oxygen species are essential for PI3K/Akt/mTOR-dependent IL-7-mediated viability of T-cell acute lymphoblastic leukemia cells. Leukemia 25, 960-967.

Skovsgaard, T. (1977). Transport and binding of daunorubicin, adriamycin, and rubidazone in Ehrlich ascites tumour cells. Biochem. Pharmacol. 26, 215-222.

Stadtman, E.R. (1990). Metal ion-catalyzed oxidation of proteins: Biochemical mechanism and biological consequences. Free Radic. Biol. Med. 9, 315-325.

Steinherz, L.J. (1991). Cardiac toxicity 4 to 20 years after completing anthracycline therapy. J. Am. Med. Assoc. 266, 1672.

Streckfuss-Bömeke, K., Tiburcy, M., Fomin, A., Luo, X., Li, W., Fischer, C., Özcelik, C., Perrot, A., Sossalla, S., Haas, J., et al. (2017). Severe DCM phenotype of patient harboring RBM20 mutation S635A can be modeled by patient-specific induced pluripotent stem cell-derived cardiomyocytes. J. Mol. Cell. Cardiol. 113, 9-21.

Sun, N., Yazawa, M., Liu, J., Han, L., Sanchez-Freire, V., Abilez, O.J., Navarrete, E.G., Hu, S., Wang, L., Lee, A., et al. (2012). Patient-specific induced pluripotent stem cells as a model for familial dilated cardiomyopathy. Sci. Transl. Med. 4, 130ra47.

Swain, S.M., Whaley, F.S., Gerber, M.C., Weisberg, S., York, M., Spicer, D., Jones, S.E., Wadler, S., Desai, A., Vogel, C., et al. (1997). Cardioprotection with dexrazoxane for doxorubicin-containing therapy in advanced breast cancer. J. Clin. Oncol. 15, 1318-1332.

Swain, S.M., Whaley, F.S., and Ewer, M.S. (2003). Congestive heart failure in patients treated with 
doxorubicin: A retrospective analysis of three trials. Cancer 97, 2869-2879.

Swenson, C.E., Bolcsak, L.E., Batist, G., Guthrie, T.H., Tkaczuk, K.H., Boxenbaum, H., Welles, L., Chow, S.C., Bhamra, R., and Chaikin, P. (2003). Pharmacokinetics of doxorubicin administered i.v. as Myocet (TLC D-99; liposome-encapsulated doxorubicin citrate) compared with conventional doxorubicin when given in combination with cyclophosphamide in patients with metastatic breast cancer. Anticancer. Drugs 14, 239-246.

Taffoni, C., Omi, S., Huber, C., Mailfert, S., Fallet, M., Ewbank, J.J., and Pujol, N. (2019). Microtubule plus-end dynamics link wound repair to the innate immune response. BioRxiv 512632.

Takahashi, K., and Yamanaka, S. (2006). Induction of pluripotent stem cells from mouse embryonic and adult fibroblast cultures by defined factors. Cell 126, 663-676.

Takahashi, K., and Yamanaka, S. (2016). A decade of transcription factor-mediated reprogramming to pluripotency. Nat. Rev. Mol. Cell Biol. 17, 183-193.

Takahashi, K., Tanabe, K., Ohnuki, M., Narita, M., Ichisaka, T., Tomoda, K., and Yamanaka, S. (2007). Induction of pluripotent stem cells from adult human fibroblasts by defined factors. Cell 131, 861872.

Tan, C., Tasaka, H., Yu, K.-P., Murphy, M.L., and Karnofsky, D.A. (1967). Daunomycin, an antitumor antibiotic, in the treatment of neoplastic disease. Cancer 20,333-353.

Taymaz-Nikerel, H., Karabekmez, M.E., Eraslan, S., and Kırdar, B. (2018). Doxorubicin induces an extensive transcriptional and metabolic rewiring in yeast cells. Sci. Rep. 8, 13672.

Tewey, K.M., Rowe, T.C., Yang, L., Halligan, B.D., and Liu, L.F. (1984). Adriamycin-induced DNA damage mediated by mammalian DNA topoisomerase II. Science 226, 466-468.

Thavendiranathan, P., Poulin, F., Lim, K.D., Plana, J.C., Woo, A., and Marwick, T.H. (2014). Use of myocardial strain imaging by echocardiography for the early detection of cardiotoxicity in patients during and after cancer chemotherapy: A systematic review. J. Am. Coll. Cardiol. 63, 2751-2768.

Tohyama, S., Hattori, F., Sano, M., Hishiki, T., Nagahata, Y., Matsuura, T., Hashimoto, H., Suzuki, T., Yamashita, H., Satoh, Y., et al. (2013). Distinct metabolic flow enables large-scale purification of mouse and human pluripotent stem cell-derived cardiomyocytes. Cell Stem Cell 12, 127-137.

du Toit, A., Hofmeyr, J.-H.S., Gniadek, T.J., and Loos, B. (2018). Measuring autophagosome flux. Autophagy 1-12.

Towbin, H., Staehelin, T., and Gordon, J. (1979). Electrophoretic transfer of proteins from polyacrylamide gels to nitrocellulose sheets: procedure and some applications. Proc. Natl. Acad. Sci. U. S. A. 76, 4350-4354.

Tripaydonis, A., Conyers, R., and Elliott, D.A. (2019). Pediatric anthracycline-induced cardiotoxicity: mechanisms, pharmacogenomics, and pluripotent stem-cell modeling. Clin. Pharmacol. Ther. 105, 614-624.

Tsialikas, J., and Romer-Seibert, J. (2015). LIN28: Roles and regulation in development and beyond. Dev. 142, 2397-2404.

Vejpongsa, P., and Yeh, E.T.H. (2014). Prevention of anthracycline-induced cardiotoxicity. J. Am. Coll. Cardiol. 64, 938-945.

Vesell, E.S., and Passananti, G.T. (1971). Utility of clinical chemical determinations of drug concentrations in biological fluids. Clin. Chem. 17, 851-866.

Visscher, H., Rassekh, S.R., Sandor, G.S., Caron, H.N., van Dalen, E.C., Kremer, L.C., van der Pal, H.J., Rogers, P.C., Rieder, M.J., Carleton, B.C., et al. (2015). Genetic variants in SLC22A17 and SLC22A7 are 
associated with anthracycline-induced cardiotoxicity in children. Pharmacogenomics 16, 1065-1076. van der Vliet, A. (2008). NADPH oxidases in lung biology and pathology: Host defense enzymes, and more. Free Radic. Biol. Med. 44, 938-955.

Volkova, M., and Russell, R. (2011). Anthracycline cardiotoxicity: Prevalence, pathogenesis and treatment. Curr. Cardiol. Rev. 7, 214-220.

Völler, S., Boos, J., Krischke, M., Würthwein, G., Kontny, N.E., Boddy, A. V., and Hempel, G. (2015). Age-dependent pharmacokinetics of doxorubicin in children with cancer. Clin. Pharmacokinet. 54, 1139-1149.

Vulsteke, C., Pfeil, A.M., Maggen, C., Schwenkglenks, M., Pettengell, R., Szucs, T.D., Lambrechts, D., Dieudonné, A.S., Hatse, S., Neven, P., et al. (2015). Clinical and genetic risk factors for epirubicininduced cardiac toxicity in early breast cancer patients. Breast Cancer Res. Treat. 152, 67-76.

Wallace, K.B. (2003). Doxorubicin-induced cardiac mitochondrionopathy. Pharmacol. Toxicol. 93, 105-115.

Wang, X., and Proud, C.G. (2006). The mTOR pathway in the control of protein synthesis. Physiology $21,362-369$.

Wang, F., Iskra, B., Kleinerman, E., Alvarez-Florez, C., Andrews, T., Shaw, A., Chandra, J., Schadler, K., and Aune, G.J. (2018). Aerobic exercise during early murine doxorubicin exposure mitigates cardiac toxicity. J. Pediatr. Hematol. Oncol. 40, 208-215.

Wang, Z., Wang, L., Liu, W., Hu, D., Gao, Y., Ge, Q., Liu, X., Li, L., Wang, Y., Wang, S., et al. (2019). Pathogenic mechanism and gene correction for LQTS-causing double mutations in KCNQ1 using a pluripotent stem cell model. Stem Cell Res. 38, 101483.

Wasowska, M., Oszczapowicz, I., Wietrzyk, J., Opolski, A., Madej, J., Dzimira, S., and Oszczapowicz, J. (2005). Influence of the structure of new anthracycline antibiotics on their biological properties. Anticancer Res. 25, 2043-2048.

White, S.J., Kasman, L.M., Kelly, M.M., Lu, P., Spruill, L., McDermott, P.J., and Voelkel-Johnson, C. (2007). Doxorubicin generates a proapoptotic phenotype by phosphorylation of elongation factor 2 . Free Radic. Biol. Med. 43, 1313-1321.

Wojnowski, L., Kulle, B., Schirmer, M., Schlüter, G., Schmidt, A., Rosenberger, A., Vonhof, S., Bickeböller, H., Toliat, M.R., Suk, E.-K., et al. (2005). NAD(P)H oxidase and multidrug resistance protein genetic polymorphisms are associated with doxorubicin-induced cardiotoxicity. Circulation $112,3754-3762$.

Wood, M.J., Irwin, W.J., and Scott, D.K. (1990). Photodegradation of doxorubicin, daunorubicin and epirubicin measured by high-performance liquid chromatography. J. Clin. Pharm. Ther. 15, 291-300.

Xing, M., Yan, F., Yu, S., and Shen, P. (2015). Efficacy and cardiotoxicity of liposomal doxorubicinbased chemotherapy in advanced breast cancer: A meta-analysis of ten randomized controlled trials. PLoS One 10, e0133569.

$\mathrm{Xu}, \mathrm{X} ., \mathrm{Persson}, \mathrm{H.L.}$, and Richardson, D.R. (2005). Molecular pharmacology of the interaction of anthracyclines with iron. Mol. Pharmacol. 68, 261-271.

Xu, X., Bucala, R., and Ren, J. (2013). Macrophage migration inhibitory factor deficiency augments doxorubicin-induced cardiomyopathy. J. Am. Heart Assoc. 2, e000439.

Yan, J., Zhou, Y., Chen, D.X., Li, L.L., Yang, X., You, Y., and Ling, X. (2015). Effects of mitochondrial translocation of telomerase on drug resistance in hepatocellular carcinoma cells. J. Cancer 6, 151159. 
Yancy, C.W., Jessup, M., Bozkurt, B., Butler, J., Casey, D.E., Drazner, M.H., Fonarow, G.C., Geraci, S.A., Horwich, T., Januzzi, J.L., et al. (2013). 2013 ACCF/AHA guideline for the management of heart failure: Executive summary: A report of the American college of cardiology foundation/American Heart Association task force on practice guidelines. Circulation 128, 1810-1852.

Yancy, C.W., Jessup, M., Bozkurt, B., Butler, J., Casey, D.E., Colvin, M.M., Drazner, M.H., Filippatos, G.S., Fonarow, G.C., Givertz, M.M., et al. (2017). 2017 ACC/AHA/HFSA Focused Update of the 2013 ACCF/AHA Guideline for the Management of Heart Failure: A Report of the American College of Cardiology/American Heart Association Task Force on Clinical Practice Guidelines and the Heart Failure Society of Amer. J. Am. Coll. Cardiol. 70, 776-803.

Yeh, J.K., and Wang, C.Y. (2016). Telomeres and telomerase in cardiovascular diseases. Genes (Basel). $7,58$.

Yoshida, Y., and Yamanaka, S. (2017). Induced pluripotent stem cells 10 years later. Circ. Res. 120, 1958-1968.

Yoshii, S.R., and Mizushima, N. (2017). Monitoring and measuring autophagy. Int. J. Mol. Sci. 18, 1865.

Yu, J., Vodyanik, M.A., Smuga-Otto, K., Antosiewicz-Bourget, J., Frane, J.L., Tian, S., Nie, J., Jonsdottir, G.A., Ruotti, V., Stewart, R., et al. (2007). Induced pluripotent stem cell lines derived from human somatic cells. Science 318, 1917-1920.

Yu, J.M., Li, W.D., Lu, L., Zhou, X.Y., Wang, D.Y., Li, H.M., Xu, X.Y., and Chen, J. (2014). Preparation and characterization of galactosylated glycol chitosan micelles and its potential use for hepatomatargeting delivery of doxorubicin. J. Mater. Sci. Mater. Med. 25, 691-701.

Zamorano, J.L., Lancellotti, P., Muñoz, D.R., Aboyans, V., Asteggiano, R., Galderisi, M., Habib, G., Lenihan, D.J., Lip, G.Y.H., Lyon, A.R., et al. (2017). 2016 ESC position paper on cancer treatments and cardiovascular toxicity developed under the auspices of the ESC committee for practice guidelines: The task force for cancer treatments and cardiovascular toxicity of the european society of cardiology. Russ. J. Cardiol. 143, 105-139.

Zaunbrecher, R.J., Abel, A.N., Beussman, K., Leonard, A., von Frieling-Salewsky, M., Fields, P.A., Pabon, L., Reinecke, H., Yang, X., Macadangdang, J., et al. (2019). Cronos titin is expressed in human cardiomyocytes and necessary for normal sarcomere function. Circulation. (in print).

von Zglinicki, T. (2002). Oxidative stress shortens telomeres. Trends Biochem. Sci. 27, 339-344.

von Zglinicki, T. (2006). Role of oxidative stress in telomere length regulation and replicative senescence. Ann. N. Y. Acad. Sci. 908, 99-110.

Zhang, M., Perino, A., Ghigo, A., Hirsch, E., and Shah, A.M. (2013). NADPH oxidases in heart failure: Poachers or gamekeepers? Antioxid. Redox Signal. 18, 1024-1041.

Zhang, S., Liu, X., Bawa-Khalfe, T., Lu, L.-S., Lyu, Y.L., Liu, L.F., and Yeh, E.T. (2012). Identification of the molecular basis of doxorubicin-induced cardiotoxicity. Nat. Med. 18, 1639-1642.

Zhao, J., Zhai, B., Gygi, S.P., and Goldberg, A.L. (2015). MTOR inhibition activates overall protein degradation by the ubiquitin proteasome system as well as by autophagy. Proc. Natl. Acad. Sci. U. S. A. $112,15790-15797$.

Zhao, Y., McLaughlin, D., Robinson, E., Harvey, A.P., Hookham, M.B., Shah, A.M., McDermott, B.J., and Grieve, D.J. (2010). Nox2 NADPH oxidase promotes pathologic cardiac remodeling associated with doxorubicin chemotherapy. Cancer Res. 70, 9287-9297.

Zhou, M., Diwu, Z., Panchuk-Voloshina, N., and Haugland, R.P. (1997). A stable nonfluorescent derivative of resorufin for the fluorometric determination of trace hydrogen peroxide: Applications in 
detecting the activity of phagocyte NADPH oxidase and other oxidases. Anal. Biochem. 253, 162-168. Zhu, W., Soonpaa, M.H., Chen, H., Shen, W., Payne, R.M., Liechty, E.A., Caldwell, R.L., Shou, W., and Field, L.J. (2009). Acute doxorubicin cardiotoxicity is associated with p53-induced inhibition of the mammalian target of rapamycin pathway. Circulation 119, 99-106. 
Appendix

Blot 2

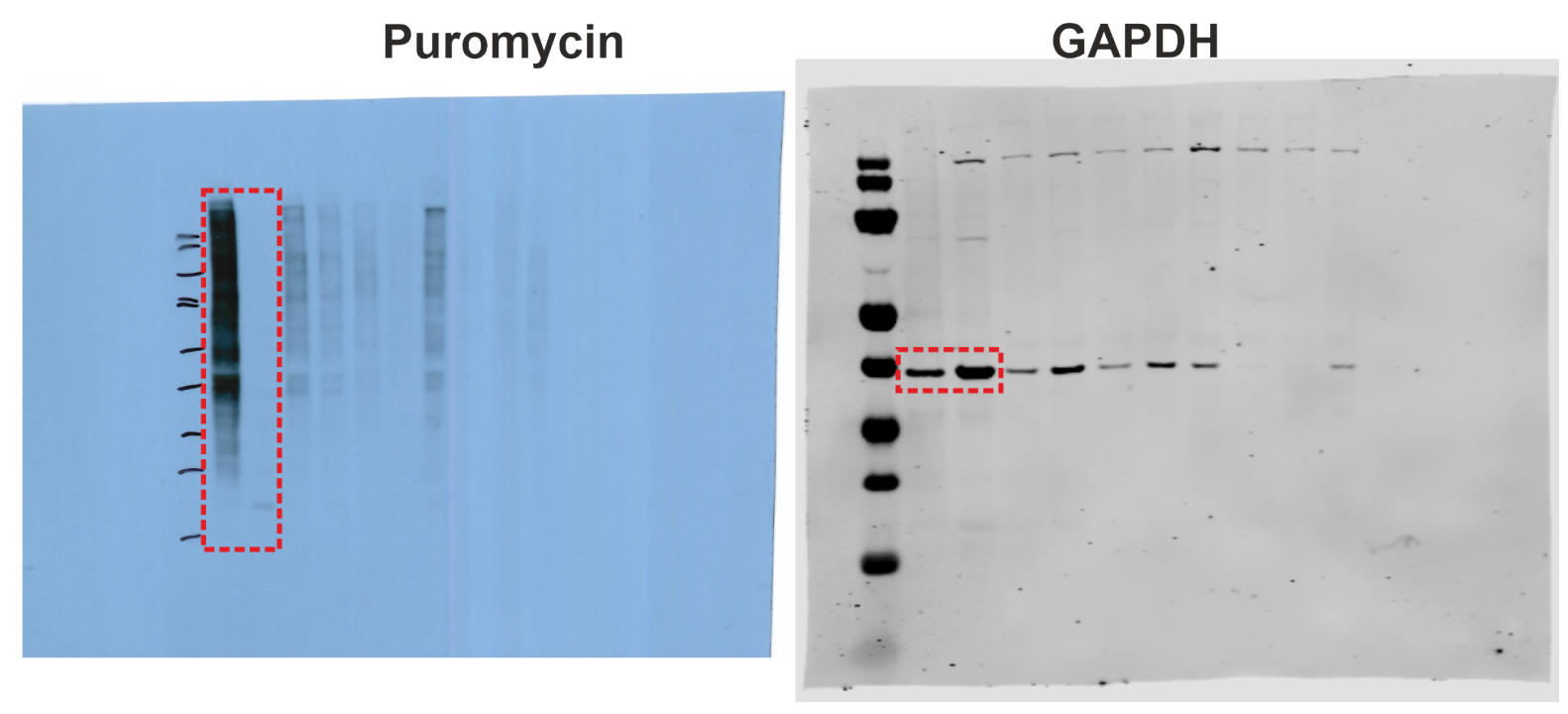

Blot 4

Puromycin

Gel 1

Gel 2
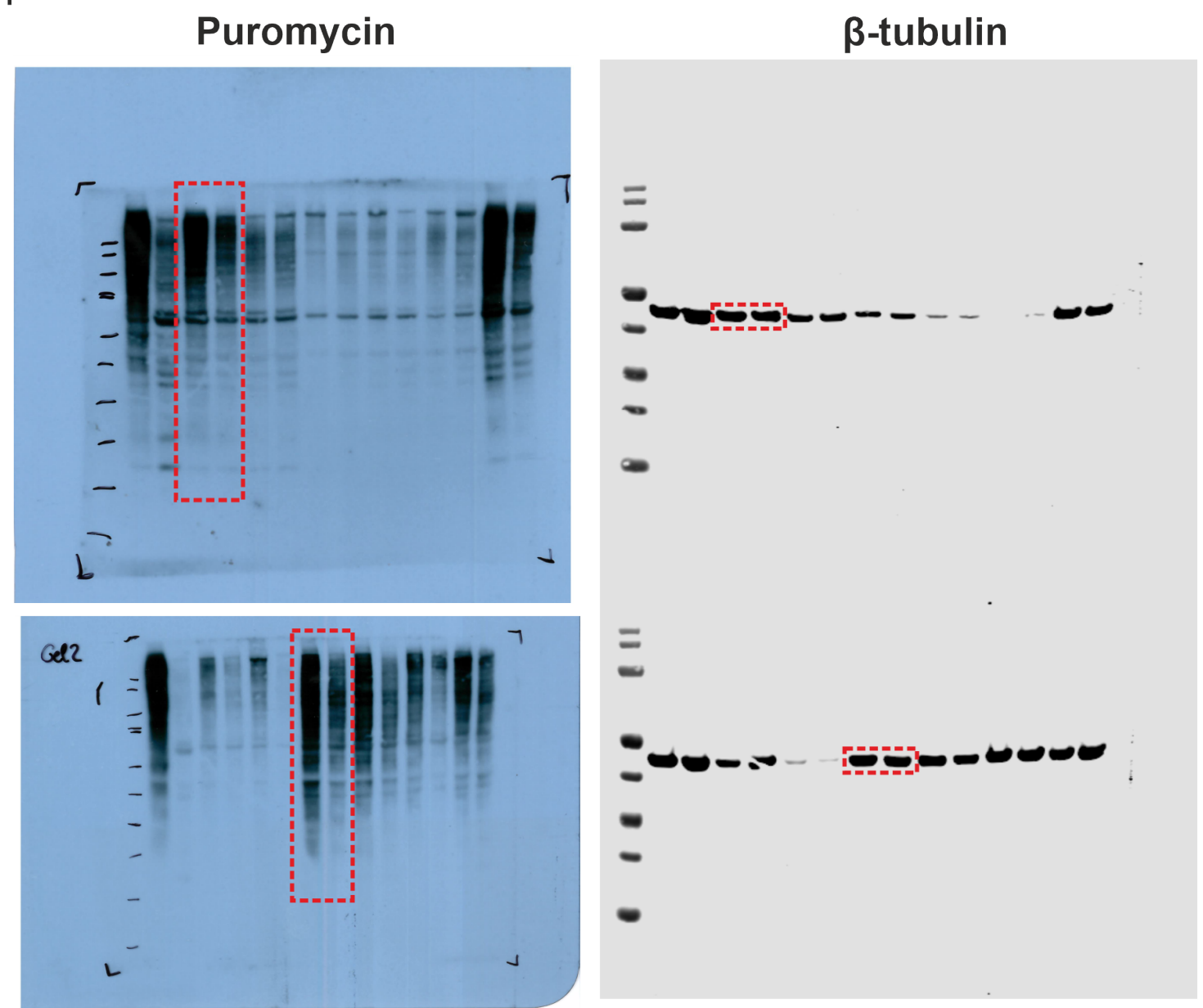


\section{Blot 5}

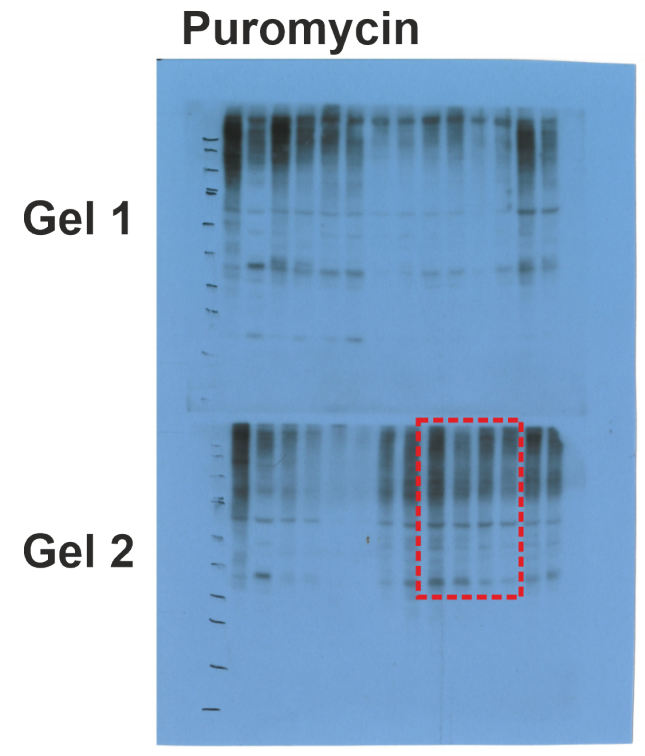

Exposure 1

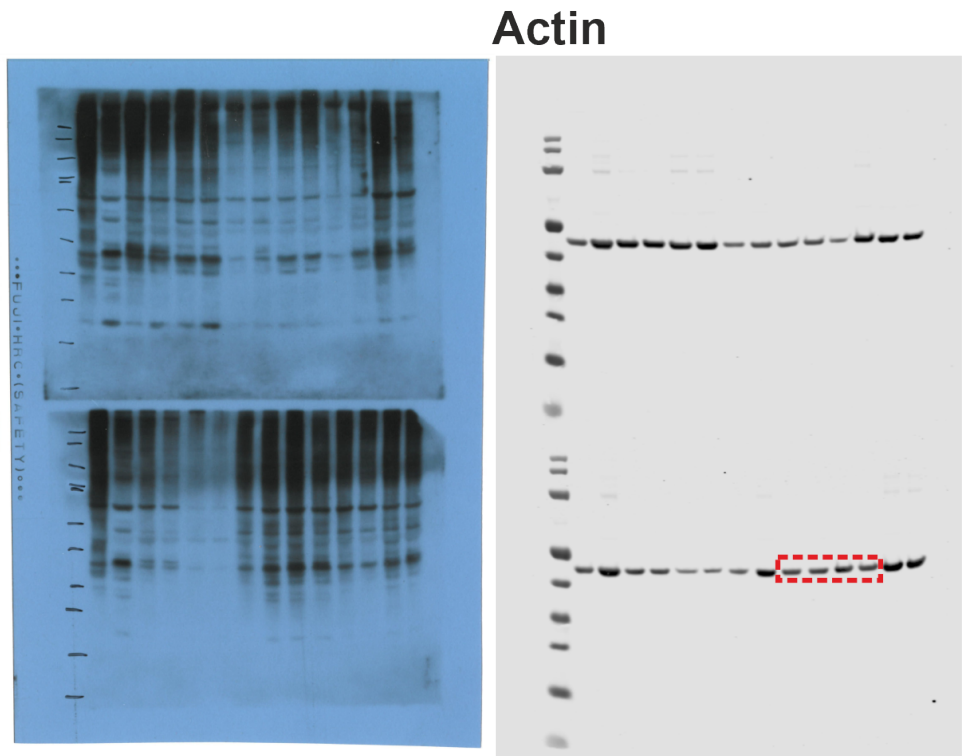

Exposure 2 
Detailed values of statistical analyses

\begin{tabular}{|c|c|c|c|c|c|c|}
\hline Figure & Comparison & Statistical test & Correction & $\begin{array}{l}\text { Mean } \\
\text { difference }\end{array}$ & $\begin{array}{l}\text { Confidence } \\
\text { interval (95\%) }\end{array}$ & $P$ value \\
\hline $5-1 \mathrm{~B}$ & $\begin{array}{l}\text { ACT untr. vs ACT } \\
1 \mu \mathrm{M} D O X\end{array}$ & $\begin{array}{l}\text { Two-way ANOVA/ } \\
\text { mixed effects }\end{array}$ & Tukey & -0.9916 & -1.015 to -0.9677 & $<0.001$ \\
\hline $5-1 B$ & $\begin{array}{l}\text { ACT } 1 \mu \mathrm{M} \text { DOX vs } \\
\text { ACT } 5 \mu \mathrm{M} \text { DOX }\end{array}$ & $\begin{array}{l}\text { Two-way ANOVA/ } \\
\text { mixed effects }\end{array}$ & Tukey & -3.719 & -6.195 to -1.243 & 0.02 \\
\hline $5-1 \mathrm{~B}$ & $\begin{array}{l}\text { ACT } 5 \mu \mathrm{M} \text { DOX vs } \\
\text { control } 5 \mu \mathrm{M} \text { DOX }\end{array}$ & $\begin{array}{l}\text { Two-way ANOVA/ } \\
\text { mixed effects }\end{array}$ & Sidak & -3.519 & -4.660 to -2.378 & $<0.001$ \\
\hline $5-2 A$ & $\begin{array}{l}\text { Control untr. vs } \\
\text { control } 2 \mathrm{~h}\end{array}$ & $\begin{array}{l}\text { Two-way ANOVA/ } \\
\text { mixed effects }\end{array}$ & Tukey & -17465 & -27490 to -7440 & 0.0105 \\
\hline $5-2 A$ & $\begin{array}{l}\text { Control untr. vs } \\
\text { control } 6 \mathrm{~h}\end{array}$ & $\begin{array}{l}\text { Two-way ANOVA/ } \\
\text { mixed effects }\end{array}$ & Tukey & -29841 & -54106 to -5576 & 0.0283 \\
\hline $5-2 B$ & $\begin{array}{l}\text { ACT untr. vs ACT } \\
24 \mathrm{~h}\end{array}$ & $\begin{array}{l}\text { Two-way ANOVA/ } \\
\text { mixed effects }\end{array}$ & Tukey & 0.9935 & 0.9708 to 1.016 & $<0.001$ \\
\hline $5-2 B$ & $\begin{array}{l}\text { Control untr. vs } \\
\text { control } 24 \mathrm{~h}\end{array}$ & $\begin{array}{l}\text { Two-way ANOVA/ } \\
\text { mixed effects }\end{array}$ & Tukey & 0.9862 & 0.9248 to 1.048 & $<0.001$ \\
\hline $5-3 C$ & ACT 0 vs ACT 7 & $\begin{array}{l}\text { Two-way ANOVA/ } \\
\text { mixed effects }\end{array}$ & Tukey & 0.8268 & 0.3199 to 1.334 & 0.0132 \\
\hline $5-3 C$ & $\begin{array}{l}\text { Control } 0 \text { vs control } \\
3\end{array}$ & $\begin{array}{l}\text { Two-way ANOVA/ } \\
\text { mixed effects }\end{array}$ & Tukey & 0.7491 & 0.2138 to 1.284 & 0.0262 \\
\hline $5-3 C$ & $\begin{array}{l}\text { Control } 0 \text { vs control } \\
7\end{array}$ & $\begin{array}{l}\text { Two-way ANOVA/ } \\
\text { mixed effects }\end{array}$ & Tukey & 0.9704 & 0.8962 to 1.045 & $<0.001$ \\
\hline $5-4 A$ & $\begin{array}{l}\text { Mito vs. membrane } \\
\text { fraction }\end{array}$ & Two-way ANOVA & Tukey & 164608 & 4004 to 325212 & 0.04 \\
\hline $5-6 C$ & $\begin{array}{l}\text { ACT untr. vs ACT } \\
1 \mu \mathrm{M} \text { DOX }\end{array}$ & Two-way ANOVA & $\mathrm{n} / \mathrm{a}$ & 0.2043 & $\begin{array}{l}0.01019 \text { to } \\
0.3984\end{array}$ & 0.04 \\
\hline $5-6 C$ & $\begin{array}{l}\text { Control untr. vs } \\
\text { control } 1 \mu \mathrm{M} \text { DOX }\end{array}$ & Two-way ANOVA & $\mathrm{n} / \mathrm{a}$ & 0.2566 & $\begin{array}{l}0.04392 \text { to } \\
0.4692\end{array}$ & 0.02 \\
\hline $5-6 D$ & $\begin{array}{l}\text { Untreated vs. } 1 \mu \mathrm{M} \\
\text { DOX }\end{array}$ & Two-way ANOVA & $\mathrm{n} / \mathrm{a}$ & 0.2205 & $\begin{array}{l}0.08777 \text { to } \\
0.3532\end{array}$ & 0.005 \\
\hline $5-14 \mathrm{~A}$ & $\begin{array}{l}\text { qPCR: iPSC vs } \\
\text { iPSC-CM }\end{array}$ & $\begin{array}{l}\text { Unpaired t-test. } \\
\text { two-tailed }\end{array}$ & $\mathrm{n} / \mathrm{a}$ & $\begin{array}{l}-0.2237 \pm \\
0.06419\end{array}$ & -0.3808 to 0.0666 & 0.01 \\
\hline $5-14 \mathrm{~B}$ & $\begin{array}{l}\text { qFISH: iPSC vs } \\
\text { iPSC-CM }\end{array}$ & $\begin{array}{l}\text { Unpaired t-test. } \\
\text { two-tailed }\end{array}$ & $\mathrm{n} / \mathrm{a}$ & $\begin{array}{l}-2494 \pm \\
815.5\end{array}$ & -4423 to -566.0 & 0.02 \\
\hline $5-20 A$ & $\begin{array}{l}\text { Blank untr. vs } \\
\text { blank } 0.5 \mu \mathrm{M} \text { DOX }\end{array}$ & Two-way ANOVA & Sidak & -389160 & $\begin{array}{l}-547833 \text { to }- \\
230486 \\
\end{array}$ & $<0.001$ \\
\hline $5-20 A$ & $\begin{array}{l}\text { Empty AAV6 untr. } \\
\text { vs } \\
\text { Empty AAV6 } \\
0.5 \text { MM DOX }\end{array}$ & Two-way ANOVA & Sidak & -247292 & $\begin{array}{l}-405966 \text { to }- \\
88619\end{array}$ & 0.007 \\
\hline $5-20 A$ & $\begin{array}{l}\text { AAV6 TERT untr. } \\
\text { vs } \\
\text { AAV6 TERT } \\
0.5 \mu \mathrm{M} \text { DOX }\end{array}$ & Two-way ANOVA & Sidak & -319118 & $\begin{array}{l}-477791 \text { to }- \\
160445\end{array}$ & 0.002 \\
\hline $5-20 B$ & $\begin{array}{l}\text { Blank untr. vs } \\
\text { blank } 0.5 \mu \mathrm{M} \text { DOX }\end{array}$ & Two-way ANOVA & Sidak & -0.5509 & $\begin{array}{l}-0.7811 \text { to }- \\
0.3207\end{array}$ & $<0.001$ \\
\hline $5-20 B$ & $\begin{array}{l}\text { Empty AAV6 untr. } \\
\text { vs } \\
\text { Empty AAV6 } \\
0.5 \mu \mathrm{M} \text { DOX }\end{array}$ & Two-way ANOVA & Sidak & -0.3552 & $\begin{array}{l}-0.5854 \text { to }- \\
0.1250\end{array}$ & 0.007 \\
\hline $5-20 B$ & $\begin{array}{l}\text { AAV6 TERT untr. } \\
\text { vs } \\
\text { AAV6 TERT } \\
0.5 \mu \mathrm{M} \text { DOX }\end{array}$ & Two-way ANOVA & Sidak & -0.3539 & $\begin{array}{l}-0.5841 \text { to }- \\
0.1237\end{array}$ & 0.007 \\
\hline $\begin{array}{l}5-25 \mathrm{~B} \\
\text { Volume }\end{array}$ & CRT 1 vs DCM 1 & One-way ANOVA & Tukey & 2535 & 2125 to 2944 & $<0.001$ \\
\hline $\begin{array}{l}5-25 \mathrm{~B} \\
\text { Volume }\end{array}$ & CRT 1 vs DCM 3 & One-way ANOVA & Tukey & -811.1 & -1221 to -401.2 & $<0.001$ \\
\hline $\begin{array}{l}5-25 \mathrm{~B} \\
\text { Volume }\end{array}$ & DCM 1 vs DCM 3 & One-way ANOVA & Tukey & -3346 & -3712 to -2979 & $<0.001$ \\
\hline $\begin{array}{l}\text { 5-25B } \\
\text { Diameter }\end{array}$ & CRT 1 vs DCM 1 & One-way ANOVA & Tukey & 2.713 & 2.287 to 3.138 & $<0.001$ \\
\hline
\end{tabular}




\begin{tabular}{|c|c|c|c|c|c|c|}
\hline $\begin{array}{l}\text { 5-25B } \\
\text { Diameter }\end{array}$ & CRT 1 vs DCM 3 & One-way ANOVA & Tukey & -0.7417 & -1.167 to -0.3162 & $<0.001$ \\
\hline $\begin{array}{l}\text { 5-25B } \\
\text { Diameter }\end{array}$ & DCM 1 vs DCM 3 & One-way ANOVA & Tukey & -3.454 & -3.835 to -3.074 & $<0.001$ \\
\hline $5-27 A$ & $\begin{array}{l}\text { DCM 3. } 0.25 \mu \mathrm{M} \\
\text { DOX vs DCM } 3 . \\
1 \mu \mathrm{M} \text { DOX }\end{array}$ & One-way ANOVA & Tukey & 0.04915 & $\begin{array}{l}0.001902 \text { to } \\
0.09639\end{array}$ & 0.04 \\
\hline $5-27 C$ & $\begin{array}{l}\text { Crt-DCM 2. untr. vs } \\
\text { DCM 1. untr. }\end{array}$ & One-way ANOVA & Tukey & 7.442 & 5.845 to 9.040 & $<0.001$ \\
\hline $5-27 C$ & $\begin{array}{l}\text { Crt-DCM } 2 \text { untr. vs } \\
\text { DCM } 3 \text { untr. }\end{array}$ & One-way ANOVA & Tukey & 8.580 & 6.971 to 10.19 & $<0.001$ \\
\hline $5-27 C$ & $\begin{array}{l}\text { Crt-DCM } 2 . \\
0.25 \mu \mathrm{M} \text { DOX vs } \\
\text { DCM 1.0.25 } \mu \mathrm{M} \\
\text { DOX }\end{array}$ & One-way ANOVA & Tukey & 6.569 & 4.871 to 8.267 & $<0.001$ \\
\hline $5-27 C$ & $\begin{array}{l}\text { Crt-DCM } 2 \text {. } \\
0.25 \mu \mathrm{M} \text { DOX vs } \\
\text { DCM 3. } 0.25 \mu \mathrm{M} \\
\text { DOX }\end{array}$ & One-way ANOVA & Tukey & 7.573 & 5.871 to 9.275 & $<0.001$ \\
\hline $5-27 C$ & $\begin{array}{l}\text { Crt-DCM 2.1 } 1 \mathrm{M} \\
\text { DOX vs } \\
\text { DCM 1. } 1 \mu \mathrm{M} \text { DOX }\end{array}$ & One-way ANOVA & Tukey & 5.943 & 4.277 to 7.609 & $<0.001$ \\
\hline $5-27 C$ & $\begin{array}{l}\text { Crt-DCM } 2.1 \mu \mathrm{M} \\
\text { DOX vs } \\
\text { DCM 3. } 1 \mu \mathrm{M} \text { DOX }\end{array}$ & One-way ANOVA & Tukey & 6.933 & 5.254 to 8.612 & $<0.001$ \\
\hline
\end{tabular}

Untr.=untreated; $n / a=$ not applicable 


\section{Acknowledgments}

A work like this one is not the accomplishment of a single individual. I therefore would like to express my sincerest gratitude to my supervisor PD Dr. Katrin Streckfuß-Bömeke for giving me the opportunity to pursue the research project in her group and for her continuous support during my PhD. I thank Prof. Dr. Gerd Hasenfuß for his support and for creating the opportunity for me to conduct research in his department. Furthermore, I would like to thank my second supervisor Prof. Dr. Ajay Shah for inviting me to work in his laboratory and for sharing his expertise with me. The guidance of my supervisors helped me in all the time of research and writing of this thesis. I am grateful for the chance to work at King's College and it would not have been as productive without Dr. Celio Santos. I thank him for his patience, motivation, and practical tips concerning lab work and beyond.

I would like to thank Prof Dr. Thomas Meyer who served as a precise reviewer, and the rest of the thesis committee, namely PD Dr. Laura Zelarayan and Prof Dr. Lutz Walter, for their insightful comments and encouragement. I would like to express my gratitude towards the other member of the examination board, PD Dr. Sven Thoms for taking the time to complete my examination board.

My sincere thanks also goes to the invaluable technicians at the stem cell lab, in particular Sandra, Johanna, and Carmen who taught me everything I know about iPSC culture and supported me in so many ways. I also would like to thank all the other members of the stem laboratory for interesting discussions, weekend services and fun lab outings.

I genuinely thank Dr. Lukas Cyganek for sharing his expertise in CRISPR/Cas9 gene-editing in iPSCs and helping us to design the approach. Furthermore, thanks to Lukas and his team at the Stem Cell Unit for letting me use their CASY cell counter.

I thank Sabrina Becker for guidance and help with the flow cytometer and the Proteomics lab at UMG for drying my samples with their speedVac. Thanks to Dr. Norman Liaw for sharing his Seahorse analyzer and helping me to set it up. A big 'thank you' to Prof. Susanne Lutz for letting me use the cellavista device and for taking the time to give me a profound induction to it.

As part of the IRTG PhD program, I had the irreplaceable opportunity to conduct parts of my research at the James Black Centre at King's College London. I am thankful towards the speakers and organizers to have created this wonderful program. My research stay was only possible, because Dr. Christina Würtz and Monica Stollery organized my travels to London and took on all bureaucratic hurdles during my stay at the James Black Center - thank you for that! 
I had the privilege to meet many amazing people at King's. In particular, I am grateful to Dr. Matteo Baretta for his precious advice, many fruitful discussions and a warm welcome at King's. A specials thanks goes to Dr. Silvia Cellone Trevelin who went out of her way to support me with experiments regarding Nox2 inhibition. Certainly, I owe a big thank you to Dr. Giulia Emanuelli for being a great colleague, the go-to-person in London and a real friend.

I also thank Dr. George Chennel and Dr. Chris Molenaar for their help with confocal microscopy as well as Dr. Xiaoke Yin for help with the HPLC.

My thank you also goes to the students who helped with the project: Hilansi, Lijun, Ulises, Vera, Anika, and Aysha.

I thank Dr. Christian Bär and Shambhabi Chatterjee for the stimulating collaboration, which got me in touch with telomere research.

I thank my fellow students Eric, Giulia, Sandip, Lisa, Irina, Sumon, and Dawid for the interesting discussions during coffee breaks and the occasional distraction during work. I do not want to picture the last years without you.

Thanks to Erinn, Lydia, Sylvia, and Jonas for proofreading my thesis.

Last but not the least, I would like to thank my true friend and beloved wife Lydia for her endless patience and support. It has been truly a team effort. I also thank my family and friends who never lost faith in my project and me and who accompanied me throughout this journey and beyond. 


\section{List of publications and presentations}

\section{Publication}

Hübscher D, Rebs S, Haupt L, Borchert T, Guessoum Cl, Treu F, Köhne S, Maus A, Hambrecht M, Sossalla S, Dressel R, Uy A, Jakob M, Hasenfuss G, and StreckfussBömeke K. A high-throughput method as a diagnostic tool for HIV detection in patientspecific induced pluripotent stem cells generated by different reprogramming methods. Stem Cells Int. 2019; 2019:1-11.

\section{Presentations}

October $11^{\text {th }} 2016$

IRTG retreat at the Brocken, Harz, Germany.

Oral presentation: Role of NADPH-oxidase subunits in the development of ACT and DCM and the use of ps-iPSC-derived cardiomyocytes and cardiac fibroblasts.

January $16^{\text {th }} 2017$

BHF Annual symposium at King's College London, UK. Poster presentation: Role of NADPH-oxidase subunits in the development of ACT and DCM.

May $15^{\text {th }} 2017$

BHF symposium at King's College London.

Poster presentation: Role of NADPH-oxidase subunits in the development of ACT and DCM.

January $24^{\text {th }} 2018$

HFA winter meeting in Les Diableres, Switzerland.

Poster presentation: The role of NADPH-oxidase subunits in the development of anthracycline-induced cardiotoxicity.

May $7^{\text {th }} 2018$

IRTG student retreat in Hannover, Germany.

Poster presentation: DOX' pharmacokinetics influence the ROS production in an iPSC-CM model.

April $7^{\text {th }} 2018$

DGK Mannheim, Germany.

Poster presentation: The role of NADPH-oxidase subunits in the development of ACT.

October $11^{\text {th }} 2018$

DGK Berlin, Germany.

Poster presentation: DOX-induced side effects and alterations in ROS production, calcium handling, and sarcomeric integrity in iPSC-derived human cardiomyocytes.*

*Awarded with the second prize of the 'Hans-Jürgen-Bretschneider' poster prize at the basic science meeting. Shared first authorship with Dr. Luis Haupt. 